\title{
Magnetic carbon nanostructures and study of their transport in microfluidic devices for hyperthermia
}

\author{
Raquel 0. Rodrigues
}

\begin{abstract}
Dissertation presented for the Ph.D. degree in the Doctoral Program in Chemical and Biological Engineering at the Faculty of Engineering, University of Porto, Portugal
\end{abstract}

Supervisor: $\quad$ Adrián M.T. Silva

Co-supervisors: Helder T. Gomes

Rui A.M.M. Lima

Associate Laboratory LSRE-LCM

Department of Chemical Engineering

SRE

Faculty of Engineering, University of Porto

ASSOCIATE LABORATORY
LABORATORY OF SEPARATION AND REACTION ENGINEERING

Portugal 

"Begin at the beginning," the King said, gravely, "and go on till you come to the end; then stop."

- Lewis Carroll, Alice in Wonderland 



\section{Acknowledgements}

First and foremost, I would like to acknowledge my supervisors for the guidance, support and encouragement that I received from them along my Ph.D. study. They taught me by example, and I feel incredible blessed to have had three role-models of ethic and commitment on them. Their high standards, encouraged creativity, autonomy and sense of free-thinking, pushed me to achieve my best. In particular, I thank Professor Adrián M.T. Silva for the insightful comments and his capacity to think out-of-the-box. A true inspiration for young researchers; to Professor Helder T. Gomes for always find time to hear my numerous thoughts and plans, giving me the encourage to execute them with autonomy and sense of responsibility; and lastly but not least, I thank Professor Rui A.M.M. Lima for his genuine kindness, joy and scientific curiosity, which has been an inspiration to me since I was his master's student.

The result of this multidisciplinary thesis work is also the product of several collaborations that enriched the quality of the work developed. As a result, I am very grateful to have them as co-authors of the research articles herein presented, namely Doctor Manuel BañobreLopez, Doctor Juan Gallo and Doctor Lorena Garcia-Hervia from INL (Braga, Portugal). Doctor Giovanni Baldi and Doctor Saer Doumett from CeRiCol (Vinci, Italy). Doctor Goran Dražić from the National Institute of Chemistry (Ljubljana, Slovenia). Doctor Isabel Ferreira and Doctor Ricardo Calhelha from CIMO (Bragança, Portugal). Professor Pedro Tavares from UTAD (Vila Real, Portugal). Professor Ali Khademhosseini and Doctor Su-Ryon Shin from HarvardMIT (Cambridge, MA, USA).

In particular, a special thanks to Doctor Giovanni Baldi, Doctor Saer Doumett and COST European Cooperation in Science and Technology, through the COST Action TD1402: Multifunctional Nanoparticles for Magnetic Hyperthermia and Indirect Radiation Therapy (RADIOMAG), for the opportunity to perform a short-term scientific mission and an advanced training at CeRiCol - Centro Ricerche Colorobbia (Vinci, Italy). These scientific experiences, one in 2016 and another in 2017, were an important boost in the quality and direction of this Ph.D. thesis.

Also, I would like to thank to Fulbright Portugal, Professor Ali Khademhosseini and Doctor Su-Ryon Shin, for giving me the opportunity to join the Khademhosseini's group and be enrolled in the development of advanced organ-on-a-chip platforms for biomedical applications. 
I thank FCT - Fundação para a Ciência e a Tecnologia, for the Ph.D. grant SFRH/BD/97658/2013, with financing from the European Social Fund (through POPH and QREN) and by national funds through FCT and MCTES - Ministério da Ciência, Tecnologia e Ensino Superior.

I acknowledge the role of the Associate Laboratory LSRE-LCM, located both at the Faculty of Engineering, University of Porto (FEUP), and at the Polytechnic Institute of Bragança (IPB), for providing the facilities, services and resources required to conduct this study. Specifically, this work was financially supported by: Project POCI-01-0145-FEDER-006984 Associate Laboratory LSRE-LCM funded by FEDER through COMPETE2020 - Programa Operacional Competitividade e Internacionalização (POCl) - and by national funds through FCT.

In IPB, a special thanks to Maria João Afonso for all the technical support that was given at the Laboratório de Processos Químicos.

At the personal level, there are numerous friends and colleagues that made this journey more pleasurable and easier. Some of them I had the luck to meet when I was abroad. Their friendship allowed me to feel at home when I was far away. Thank you to all.

Family is the most important part of us, and I cannot be more grateful that I am to have mine extraordinary parents and sisters. Their unconditional love and comfort are always a warm home to return.

Finally, to you Rui. There are no words to express the gratitude of your presence in my life. Your support, love and friendship has guided me throughout this incredible journey. Thank you to bring the best of me.

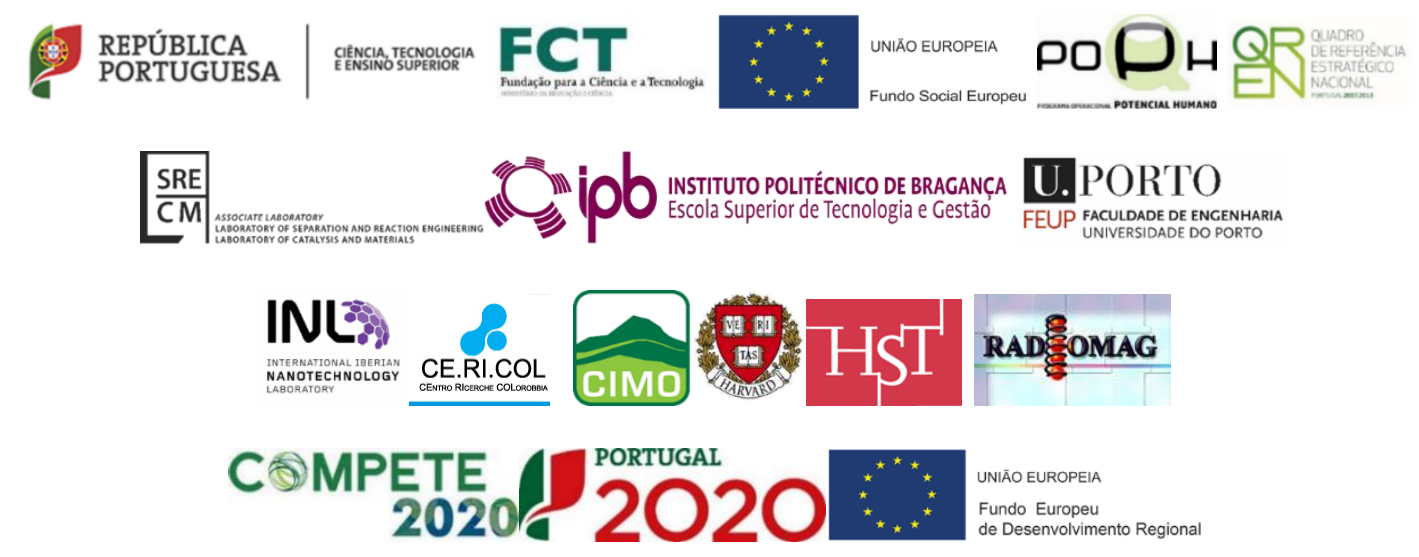




\section{Abstract}

Cancer incidence and mortality are growing worldwide at an alarming pace, emphasizing the urgent need for new strategies to combat this disease. One of the frontiers of cancer research is currently focused on the design of multifunctional magnetic nanoparticles capable to achieve the synergistic cancer theranostics (both diagnosis and therapy). Although the potentiality that these multifunctional nanosystems represents to nanomedicine and cancer treatment and diagnostic, there are still many challenges that must be addressed in a near future before this approach became a reality. The development of efficient multifunctional magnetic nanosystems able to selectively destroy cancer cells in detriment of healthy ones is one of the main challenges that have damped the spread of this technology into clinical applications. The limited biological and biophysical studies between the biomedical nanosystems and cells/tissues/organs is another challenge that has to be addressed. With these two main challenges in mind, the present Ph.D. thesis was focused on the development of:

(1) Multifunctional magnetic carbon nanostructures for the treatment of cancer:

- Exploiting the advantages of the synergism achieved at the nanoscale, namely stability and biocompatibility properties of carbon structures with the superparamagnetism of the magnetic materials, a smart and on-demand composite nanosystem, combining magnetic hyperthermia and controlled drug release, was developed for thermo-chemotherapy.

(2) New advanced microfluidic devices capable to give new insights over the developed nanosystems and human cells:

- A new microfluidic methodology based on the deformability of red blood cells was developed for the haemocompatibility assessment; this new approach is able to give new insights over the nanoparticles-blood cells interactions, when these nanosystems are in contact with blood;

- A second advanced microfluidic device based in organ-on-a-chip technology was developed as a miniaturization of 3D human organ models to recapitulate important biological and physiological parameters between nanoparticles and organs (healthy and cancer ones); for that, a novel human heart-and-breast-cancer-on-a-chip model combined with aptamer-based electrochemical biosensing to detect trace amounts of selected biomarkers secreted during chemotherapeutic treatment was developed. 
Both microfluidic platforms can be applied to the monitoring of new nanosystems developed for biomedical applications and contribute, not only to gain new insights over the complex nanoparticle-cell/tissues interactions, but also to accelerate the clinical translation of those nanomaterials. 


\section{Resumo}

O crescimento das taxas de incidência e mortalidade do cancro reforçam a necessidade urgente de encontrar novas estratégias para o combate desta doença. Atualmente, um dos ramos de investigação nesta área centra-se no desenvolvimento de nanopartículas magnéticas multifuncionais capazes de potenciar sinergias entre o diagnóstico e a terapia (teranóstico). Contudo, e embora o potencial que os nanosistemas representam para a nanomedicina e o tratamento do cancro, existem ainda muitos desafios que devem ser superados para que se torne uma realidade. Entre eles, o desenvolvimento de sistemas multifuncionais de nanopartículas magnéticas capazes de destruir seletivamente as células tumorais em detrimento das células saudáveis continua a ser um desafio. Por outro lado, a falta de estudos biológicos e biofísicos entre estes nanosistemas e as células/tecidos/órgãos humanos, também representam um desafio. Com estes dois reptos em mente, o presente estudo de doutoramento focou-se no desenvolvimento de:

(1) Nanopartículas nano-estruturadas de carbono para o tratamento multifuncional do cancro:

- Desenvolvidas tendo em atenção as vantagens sinergéticas das nano-estruturas de carbono, como a estabilidade e a biocompatibilidade, aliadas às propriedades de superparamagnetismo dos materiais magnéticos, possibilitando a formação de um compósito inteligente que combina hipertermia magnética e a libertação controlada de fármacos, a termo-quimioterapia.

(2) Novos sistemas microfluídicos capazes de elucidarem sobre o efeito de novos nanomateriais sobre as células humanas:

- Uma nova metodologia microfluídica baseada na deformação de glóbulos vermelhos foi criada para estudar o efeito hematológico dos nanomateriais no sangue;

- Um sistema microfluídico avançado baseado em órgãos-em-um-chip foi posteriormente desenvolvido com o objetivo de miniaturizar modelos 3D de órgãos humanos e recapitular parâmetros biológicos e fisiológicos existentes entre nanopartículas e órgãos (tanto saudáveis como tumorais). Para tal, foi criado um modelo humano coração-cancro-da-mamã-em-um-chip combinando a deteção de biomarcadores-chave libertados durante o tratamento de quimioterapia, através de um sistema de biossensores eletroquímicos. 
Ambas as plataformas microfluídicas desenvolvidas têm a potencialidade de serem aplicadas numa vasta gama de aplicações biomédicas para a monitorização de nanosistemas clínicos, esperando potencializar um melhor entendimento entre as complexas interações existentes entre nanopartículas-células/órgãos, e desta forma facilitar a transferência desta tecnologia para a prática clínica. 


\section{Table of Contents}

ACKNOWLEDGEMENTS

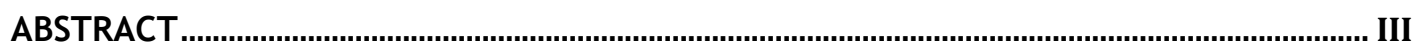

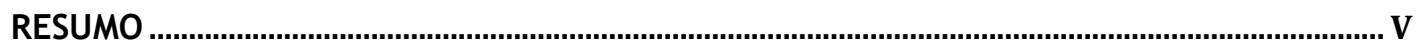

TABLE OF CONTENTS ...................................................................................................... VII

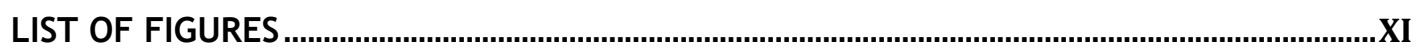

LIST OF TABLES

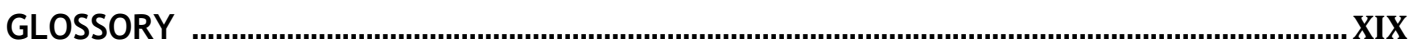

\section{CHAPTER I - INTRODUCTION}

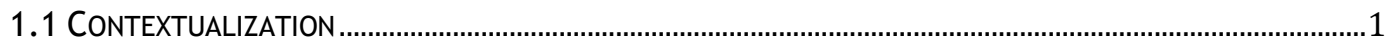

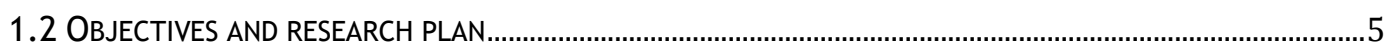

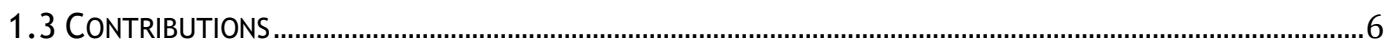

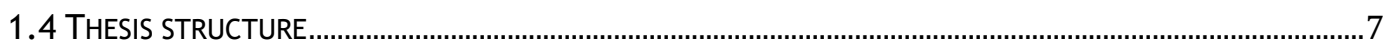

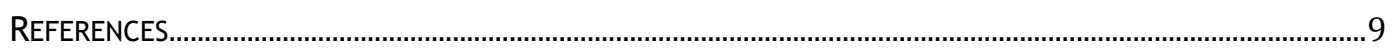

CHAPTER II - THEORETICAL CONCEPTS

2.1 MAGNETISM AND SUPERPARAMAGNETIC NANOPARTICLES .................................................................13

2.1.1 Magnetic hyperthermia, biological principle and heating mechanism .......... 15

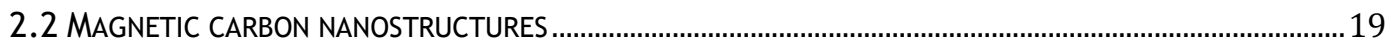

2.1.2 Graphene-based magnetic nanoparticles (GbMNPs) in nanomedicine ........... 20

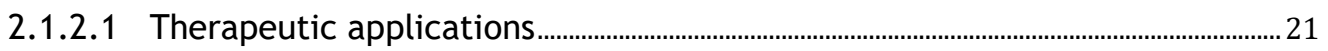

2.1.2.2 Diagnostic applications ..........................................................................................................2 24

2.2.2 Classification and synthesis techniques ................................... 25

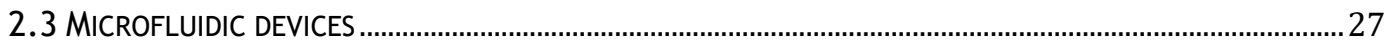

2.3.1 Microfluidics in biomedical research ..................................... 28

2.3.1.1 Single-cell biophysical studies ............................................................................................... 28

2.3.1.2 Lab-on-a-chip ...........................................................................................................................

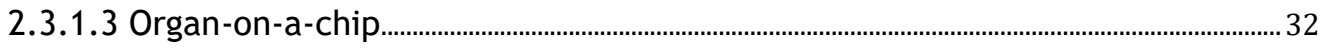

2.3.4 Microfabrication techniques of microfluidic devices ........................... 34

2.3.5 Bioanalytical analysis and applications of microfluidic devices ................ 35

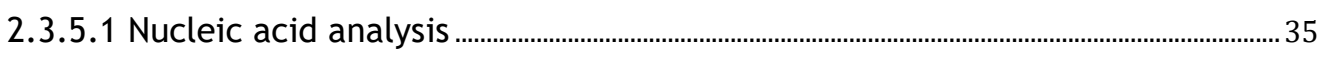

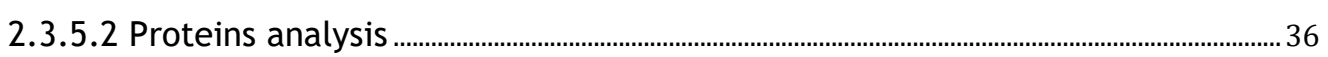




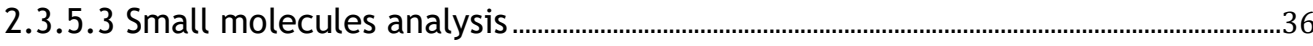

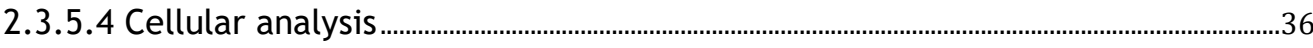

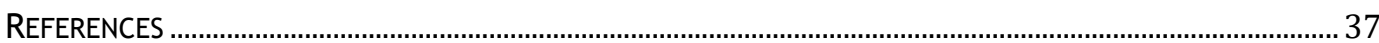

CHAPTER III - SUPERPARAMAGNETIC NANOPARTICLES FOR THERANOSTIC APPLICATIONS

3.1 INTRODUCTION. 45

3.2 HAEMOCOMPATIBILITY OF IRON OXIDE NANOPARTICLES SYNTHESIZED FOR THERANOSTIC APPLICATIONS: A

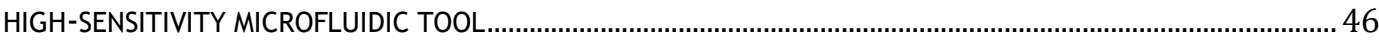

3.2.1 Materials and methods ................................................ 47

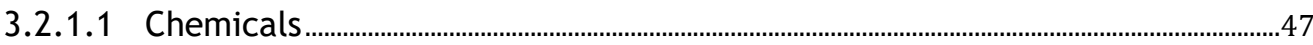

3.2.1.2 Synthesis of magnetic nanoparticles.....................................................................................

3.2.1.3 Characterization of magnetic nanoparticles ....................................................................48

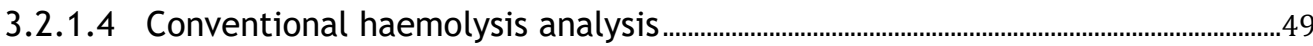

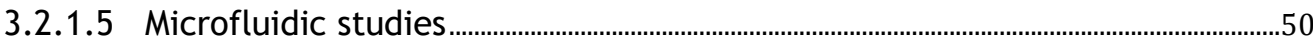

3.2.1.6 Ethical approval.....................................................................................................................52

3.2.2 Results and discussion .................................................. 52

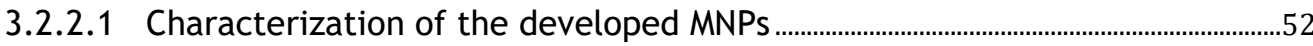

3.2.2.2 Conventional haemolysis analysis...............................................................................................57

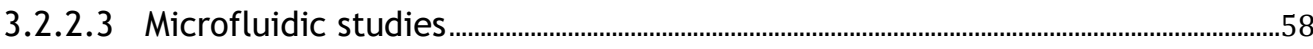

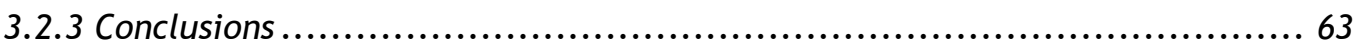

3.3 THERMAL INFRARED IMAGE PROCESSING TO ASSESS HEAT GENERATED BY MAGNETIC NANOPARTICLES FOR

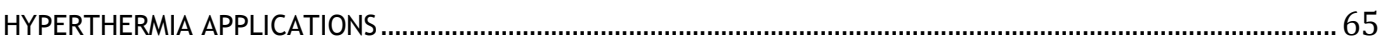

3.3.1 Materials e methods .....................................................65 65

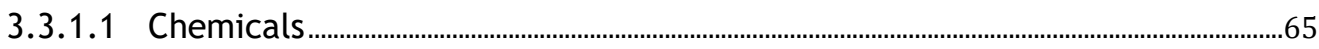

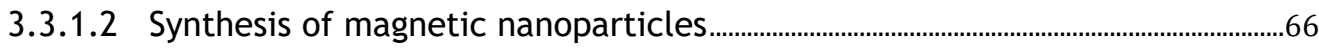

3.3.1.3 Physicochemical characterization of MNPs...................................................................66

3.3.1.4 Experimental setup........................................................................................................................66

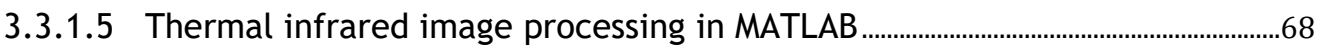

3.3.2 Results and discussion ..................................................... 70

3.3.2.1 Physicochemical characterization of MNPs.................................................................70

3.3.2.2 Calibration of Infrared camera ..................................................................................................70

3.3.2.3 Heat released by MNPs under AMF ......................................................................................72

3.3.3 Conclusions ......................................................... 73

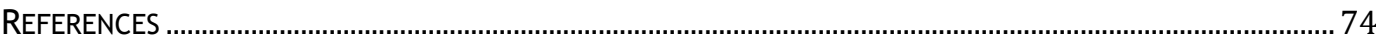

CHAPTER IV - GRAPHENE-BASED MAGNETIC NANOPARTICLES FOR THE TREATMENT OF CANCER

4. 1 INTRODUCTION

4.2 DEVELOPMENT OF A TAILOR-MADE PROTOCOL TO SYNTHESIZE GRAPHENE-BASED YOLK-SHELL MAGNETIC NANOPARTICLES FOR NANOMEDICINE 80 
4.2.1 Materials and methods ................................................... 81

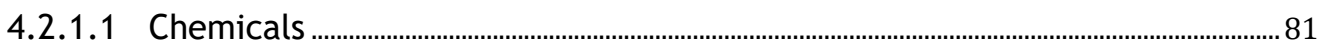

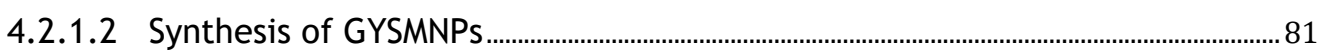

4.2.1.3 Hydrophilization of GYSMNPs................................................................................................... 82

4.2.1.4 Characterization of GYSMNPs ................................................................................................... 82

4.2.1.5 In vitro biostudies...................................................................................................................... 83

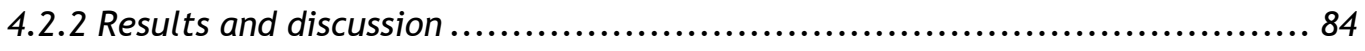

4.2.2.1 Synthesis and characterization of GYSMNPs................................................................ 84

4.2.2.2 In vitro biocompatibility assays .......................................................................................

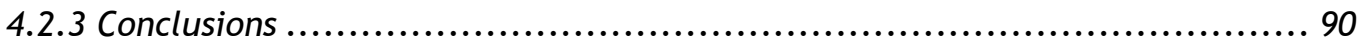

4.3 MULTIFUNCTIONAL GRAPHENE-BASED MAGNETIC NANOCARRIERS FOR COMBINED HYPERTHERMIA AND DUAL

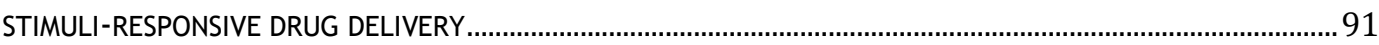

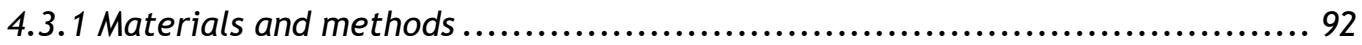

4.3.1.1 Chemicals ................................................................................................................................. 92

4.3.1.2 Synthesis and colloidal optimization of GYSMNPs ................................................... 93

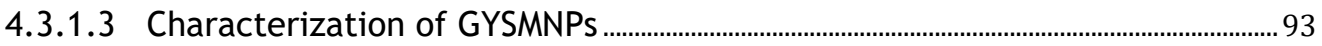

4.3.1.4 Drug loading studies ............................................................................................................. 94

4.3.1.5 pH and temperature-dependent drug release ............................................................ 94

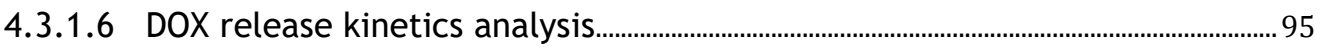

4.3.1.7......Validation of GYSMNP@PF127-DOX as dual $\mathrm{pH}$ - and AMF/temperatureresponsive drug nanocarrier .................................................................................................................. 95

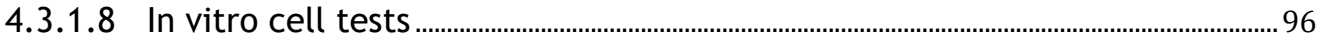

4.3.2 Results and discussion ............................................... 97

4.3.2.1 Synthesis and characterization of GYSMNP@PF127 ..................................................97

4.3.2.2 DOX loading studies ................................................................................................................... 100

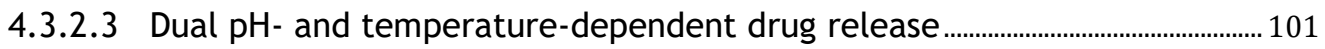

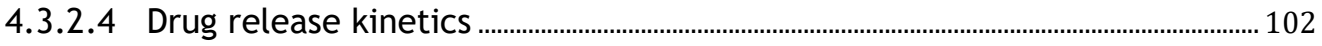

4.3.2.5 Validation of GYSMNP@PF127-DOX as dual pH- and AMF/temperature-

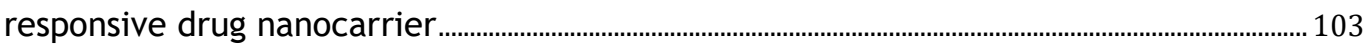

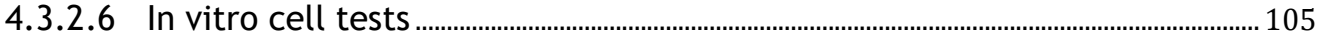

4.3 .3 Conclusions ................................................................. 107

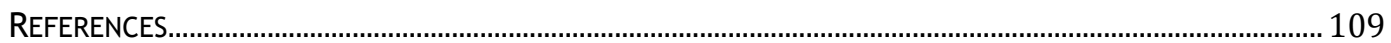

CHAPTER V - ORGAN-ON-A-CHIP PLATFORM FOR BIO-TOXICITY EVALUATION OF GRAPHENE-BASED MAGNETIC NANOPARTICLES

5.1 INTRODUCTION

5.2 DUAL-ORGAN-ON-A-CHIP PLATFORM DEVELOPED FOR DRUG SCREENING STUDIES IN NANOMEDICINE... 116

5.2.1. Experimental methods ................................................. 118

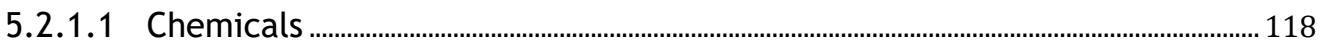

5.2.1.2 Preparation of microelectrodes................................................................................. 118 
5.2.1.3 Electrochemical measurements ....................................................................................118

5.2.1.4 Design and fabrication of dual bioreactor chips .....................................................119

5.2.1.5 Immobilization process for aptamer biosensors ........................................................119

5.2.1.6 In vitro 2D cellular studies using graphene-based magnetic nanoparticles (GYSMNP)

5.2.1.7 Culturing cardiomyocytes and breast cancer organoids in microfluidic

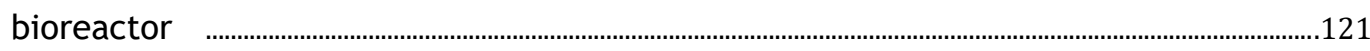

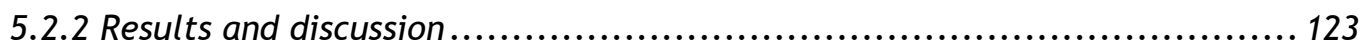

5.2.2.1 Design and calibration of a label-free EC sensing method..................................123

5.2.2.2 In vitro 2D cellular studies of drug nanocarrier GYSMNP....................................126

5.2.2.3 Monitoring of cell-secreted biomarkers from bioreactor samples..................129

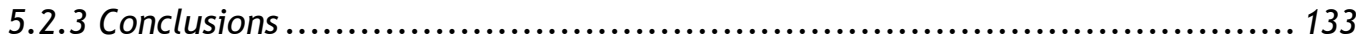

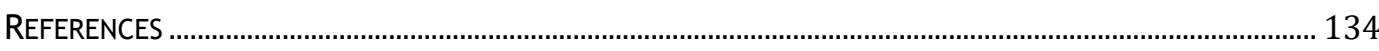

\section{CHAPTER VI - CONCLUSION AND FUTURE PERSPECTIVE}

REFERENCES

\section{APPENDIXES}

A. Deformation of Red Blood Cells, Air Bubbles, and Droplets in Microfluidic Devices: Flow VISUALIZATIONS AND MEASUREMENTS

B. A SIMPLE MICROFLUIDIC DEVICE FOR THE DEFORMABILITY ASSESSMENT OF BLOOD CELLS IN A CONTINUOUS FLOW. 161

C. POLYMER MICROFLUIDIC DEVICES: AN OVERVIEW OF FABRICATION METHODS.......................................... 170

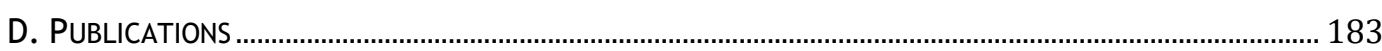

D.1 International peer reviewed ISI indexed journals ........................... 183

D.2 National peer reviewed journals .......................................... 184

D. 3 Book chapter .............................................................. 184

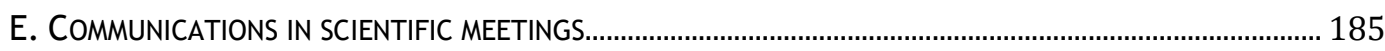

E. 1 Poster presentations ...................................................... 185

E.2 Oral presentations.................................................... 186

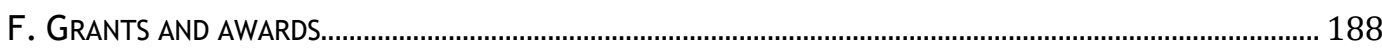




\section{List of Figures}

Figure 1.1.

Diagram of the seven main chapters that comprise the Ph.D. thesis work.

Figure 2.1.

Permeability of the magnetic flux density caused by a magnetic material, such soft iron.

Figure 2.2......

Schematic representation of a typical hysteresis loop $(\mathrm{M}-\mathrm{H})$ of: (a) multi-domain ferromagnetic material and (b) single-domain superparamagnetic material.

Figure 2.3.

Schematic chemical structures of graphene-based nanostructures: graphene, graphene oxide and reduced graphene oxide.

Figure 2.4.

Schematic diagram of possible theranostic applications, from diagnosis to therapy, by using magnetic carbon nanostructures, such as graphene-based magnetic nanoparticles.

Figure 2.5. 26

Representation of the two main general classes of magnetic carbon nanostructures: (a) carbon encapsulated MNPs (yolk-shell configuration), and (b) carbon nanostructures decorated with MNPs.

Figure 2.6.

Representation of a microfluidic device combining red blood cells separation and deformation for clinical diagnosis applications.

Figure 2.7. 30

Blood flow and RBC deformability equation used for different microfluidic induced-fluid contractions: (a) Hyperbolic contraction and (b) Sudden contraction.

Figure 2.8. 32

Images and handling procedure of i-STAT Portable Clinical Analyser system from Abbot diagnostics.

Figure 2.9.

Representation of the principal design concept and applications of organ-on-a-chip platforms.

Figure 3.1. .50

Microfluidic device fabricated in PDMS with a hyperbolic-shaped contraction to assess the haemocompatibility of the RBCs in contact with MNPs. 
Figure 3.2.

Histogram and mean diameter $(\bar{x})$ of the nanoparticles following the Log-normal distribution of $(a)$ MAG30 and (b) MAG55. Inset shows the corresponding TEM images.

Figure 3.3.

Magnetic characterization of MAG30 and MAG55. (a) Hysteresis loops at room temperature up to \pm $20 \mathrm{kOe}$; the inset is a zoom in the low-field region; (b) ZFC-FC magnetization curves measured at 50 Oe.

Figure 3.4.

Temperature versus time curves for MAG30@Al and MAG55@Al water colloidal dispersions at 0.40 $\mathrm{g}$ Fe304 $\mathrm{L}^{-1}$, under an oscillating magnetic field of $15.95 \mathrm{kA} \mathrm{m}^{-1}$ and $688 \mathrm{kHz}$.

Figure 3.5.

Microfluidic studies for the haemocompatibility of the RBCs in contact with MNPs. (a) schematic view of the experimental setup; (b) microchannel device geometry with the zoom of the hyperbolic channel; (c) graphical representation of the fluid-induced conditions profiles that occur in the hyperbolic channel, which has proven to be a more efficient way for the determination of the DI of the RBCs.

Figure 3.6.

Representation of a healthy RBC flowing along the axial position of a microchannel with a hyperbolic-shaped contraction followed by a sudden expansion, subdivided in four sections with equal distances between them $(125 \mu \mathrm{m})$. This approach allows the assessment of the RBCs' Dls, as an indicator of the physiological effect of MNPs on the RBCs membranes. Furthermore, the DI equation is represented, as well as the typical values found for a healthy RBC subjected to a hyperbolic-shaped contraction (section 2 ) and to a recuperation section (section 4), under a controlled homogeneous extensional flow field.

Figure 3.7.

Deformation index of blood cell flowing along the axial position of the hyperbolic channel, as well as the average shear rate at each hyperbolic region: (a) control RBCs versus RBCs in contact with MNPs at a final concentration of $17.4 \mu \mathrm{g}$ Fe304 $\mathrm{mL}^{-1}$ during 15, 30 and $60 \mathrm{~min}$; (b) control RBCs versus RBCs in contact with MNPs at a final concentration of $34.8 \mu \mathrm{g}$ Fe304 $\mathrm{mL}^{-1}$ during 15,30 and $60 \mathrm{~min}$. Error bars show a 95\% confidence interval ( $n=56$ blood cells).

Figure 3.8.

Experimental setup for the assessment of heat generated by MNPs under an AMF, monitored by the infrared camera (E30, FLIR, USA).

Figure 3.9.

Representation of an infrared image captured during the heat release of MNPs under an AMF: (a) thermal image obtained as .JPEG with the infrared camera; (b) thermal imaging processing showing the four regions of interest $\left(R_{1}, R_{2}, R_{3}\right.$ and $\left.R_{4}\right)$. 
Figure 3.10.

Comparison of the heat released by the different sets of synthesized MNPs $\left(5 \mathrm{mg} \mathrm{mL}^{-1}\right)$, during the three thermal assays. The mean temperatures values are corrected by the respective calibration curves calculated during the calibration of infrared camera and normalized with the heat generated by the coil (blank tests). Error bars correspond to the standard deviation obtained from the four regions of interest $\left(R_{1}-R_{4}\right)$ in each thermal experiment.

Figure 4.1.

Schematic representation of the synthesis steps involved in the development of the hydrophilic graphene-based yolk-shell magnetic nanoparticles (GYSMNPs).

Figure 4.2.

High resolution scanning transmission electron microscopy (HR-STEM) bright field images of: (a) sample GYSMNPs-1; (b) sample GYSMNPs-2; (c) sample GYSMNPs-3; (d) detail of the magnetic core of magnetite covered by few graphene layers of sample GYSMNPs-2.

Figure 4.3.

(a) Wide angle powder X-ray diffraction (XRD) patterns of $\mathrm{Fe}_{3} \mathrm{O}_{4}$ nanoparticles and GYSMNPs-2; (b) Thermogravimetric analysis of the as-synthesized graphene-based yolk-shell magnetic nanoparticles (GYSMNPs).

Figure 4.4.

Raman spectrum of as-synthesized GYSMNPs. (a) sample GYSMNPs-2 with inset corresponding to the deconvolution of the observed $D$ and apparent $G\left(G_{a p p}\right)$ bands into actual $G$ and $D$; (b) sample GYSMNPs-3 with inset corresponding to the deconvolution of the observed $D$ and $G$.

Figure 4.5.

Magnetization curves of a- Fe304, b- GYSMNPs-2 and c- GYSMNPs-3. Hysteresis loops at $27^{\circ} \mathrm{C}$ up to $\pm 20 \mathrm{kOe}$; the inset is a zoom in the low field region.

Figure 4.6.

In vitro biocompatibility study of GYSMNPs in primary cell culture cells (PLP2). Error bars represent a $95 \%$ of confidence interval.

Figure 4.7.

Schematic overview of the steps involved in the development and grafting of the hydrophilic graphene-based yolk-shell magnetic nanoparticles (GYSMNP@PF127).

Figure 4.8.

(a) High resolution transmission electron microscopy (HR-TEM) bright field images of GYSMNP with detail of the magnetite covered by few layers of the graphene-based material; (b) DLS measurements with average hydrodynamic diameter (DH). 
Figure 4.9.

(a) FTIR spectra of (i) as-synthesized GYSMNP, (ii) GYSMNP after activation with nitric acid, (iii) GYSMNP after grafting with pluronic F-127 (GYSMNP@PF127); (b) Thermogravimetric analysis of the as-synthesized and functionalized GYSMNP materials under $\mathrm{N}_{2}$ atmosphere.

Figure 4.10. 101 $\mathrm{pH}$ and thermo-responsive release of doxorubicin (DOX) from drug loaded GYSMNP@PF127 under different $\mathrm{pH}$ values $(7.4,6.0$ and 4.5$)$ and temperatures $\left(37\right.$ and $45^{\circ} \mathrm{C}$ ). Standard deviation of triplicate drug release tests $(n=3)$.

Figure 4.11. 104 Magnetic-induced thermal response curve of GYSMNP@PF127-DOX (3.0 mg GYSMNP-Dox $\mathrm{mL}^{-1}$ ) for dual pHand thermal-responsive drug delivery: (a) dispersed in phosphate buffer at 7.4 to mimic physiological microenvironment, and (b) dispersed in phosphate buffer at 4.5 to mimic tumour microenvironment. Samples were subjected to an $\operatorname{AMF}\left(\mathrm{f}=340 \mathrm{kHz}\right.$ and $\mathrm{H}=21.0 \mathrm{kA} \mathrm{m}^{-1}$ ) during $30 \mathrm{~min}$. Inset shows the drug-containing supernatant resulting from each hyperthermia experiment.

Figure 4.12. 104 Magnetic induced thermal response curve of unloaded-DOX GYSMNP@PF127 dispersed in water with a concentration of $1.5 \mathrm{mg} \mathrm{mL}^{-1}$ and subjected to an $\mathrm{AMF}$ ( $\mathrm{f}=340 \mathrm{kHz}$ and $\mathrm{H}=21.0 \mathrm{kA} \mathrm{m}^{-1}$ ).

Figure 4.13. 105 Haemolytic percentages of GYSMNP@PF127 at different concentrations ranging from 10 to 500 $\mu \mathrm{g} \mathrm{mL}{ }^{-1}$ during $3 \mathrm{~h}$ at $37^{\circ} \mathrm{C}$. Error bars represent the standard deviation of triplicate haemolysis tests of two blood donors $(n=6)$.

Figure 4.14. 106

Cell cytotoxicity assays investigated by SRB colorimetric assay after incubation of (a) GYSMNP@PF127 in healthy liver cell line PLP2; (b) GYSMNP@PF127-DOX in tumour liver cell line HepG2, under different concentrations for $48 \mathrm{~h}$.

Figure 4.15. 107

Confocal microscopy images of HepG2 cells incubated with GYSMNP@PF127-DOX at a concentration of $5 \mu \mathrm{g} \mathrm{mL}{ }^{-1}$ during $2 \mathrm{~h}(\mathrm{a}), 4 \mathrm{~h}$ (b) and $6 \mathrm{~h}$ (c). (i) Cell nuclei stained with Hoechst and excited at 405 nm; (ii) DOX released in HepG2 cells and excited at $561 \mathrm{~nm}$; (iii) Cell microtubules immunolabeled with the monoclonal anti-tubulin antibody (B512) combined with a secondary goat anti-mouse IgG antibody conjugated with Alexa Fluor 488 excited at $488 \mathrm{~nm}$; (iv) HepG2 cells using bright contrast; (v) Cell merged images.

Figure 5.1. 119 Schematic representation of the dual bioreactor chip used as microfluidic platform to contain the dual-organ system.

Figure 5.2. 
(a, b) Schematic represents the fabrication of PDMS micro-well mould for cancer cell spheroid preparation; (c) optical images of cross-sectional and top view of PDMS micro-well moulds; (d-j) Optical images of PDMS micro-wells mould at different steps: (d) after adding cell media and formation of micro-air bubbles; (e) after vacuum-removing of air bubbles; (f) seeded with $\mathrm{SkBr} 3$ cells $\left(1 \times 10^{6}\right.$ cells $\left.\mathrm{ml}^{-1}\right)$ and incubated for $30 \mathrm{~min}$ at $37^{\circ} \mathrm{C}$ with $5 \% \mathrm{CO}_{2}$; (g) washed with cell media to remove cell which were not settled into micro-wells; and ( $h-j)$ changed cell media after $24 \mathrm{~h}, 72 \mathrm{~h}$ and $96 \mathrm{~h}$ incubation at $37^{\circ} \mathrm{C}$ with $5 \% \mathrm{CO}_{2}$, respectively. Inserts show higher magnification of cancer spheroid morphologies; (k) histogram shows SkBb3 spheroid size distribution at day 4 (number of considered spheroids $=100$ ).

Figure 5.3

(a) Image of the microfabricated electrode set containing a reference electrode (RE), a counter electrode (CE) and a working electrode (WE); (b) image of a single electrode as the one used in this study; (c) schematic diagram of the immobilization steps of the aptamers into the microelectrode.

Figure 5.4.

Calibration curves and respective Nyquist curves obtained at $37{ }^{\circ} \mathrm{C}$ for (a) cardiac Troponin-T aptamer; (b) human cardiac CKMB aptamer; and (c) breast cancer HER2 aptamer.

Figure 5.5.

Confocal microscopy images of $\mathrm{SkBr} 3$ cells incubated during $24 \mathrm{~h}$ at $37{ }^{\circ} \mathrm{C}$ : (a) without chemotherapeutic treatment; (b) free DOX $(10 \mu \mathrm{M})$; and (c-e) GYSMNP@PF127-DOX at different concentration 15, 30 and $60 \mu \mathrm{g} \mathrm{mL}^{-1}$, corresponding to 10, 20, $40 \mu \mathrm{M}$ of loaded DOX, respectively. (i) Cells stained with Calcein-AM excited at $495 \mathrm{~nm}$; (ii) DOX released in $\mathrm{SkBr} 3$ cells and excited at 561 nm; (iii) cell merged images. Scale bares represent $100 \mu \mathrm{m}$.

Figure 5.6

Cell cytotoxicity assays treated with GYSMNP@PF127, free DOX and GYSMNP@PF127-DOX and investigated by PrestoBlue colorimetric assay during 5 days. (a) Breast cancer $(\mathrm{SkBr} 3)$; (b) healthy cardiomyocytes (iPSCs).

Figure 5.7

Schematic representation of the dual-organ-on-a-chip microfluidic bioreactor developed tomonitor the effect of chemotherapeutic drug Doxorubicin (free or encapsulated in nanocarriers) over the breast cancer and cardiac healthy organoids. Life/dead confocal images from iPSCs and SkBr3 spheroids showing the high viability of these organoids inside the GelMA hydrogel after UV photocross linking. Live cells stained green and dead cells stained red.

Figure 5.8

130

(a) Schematic representation of the feedback loop system used during the drug induced experiments on dual-organ-on-a-chip microfluidic bioreactor along 5 days. Electrochemical sensing of selected biomarkers released from dual organ-on-a-chip bioreactor: (b) cardiac biomarker Troponin-T; (c) cardiac biomarker CKMB; (d) breast cancer biomarker HER2. Error bars show standard deviation of 
individual single microsensors $(n=3)$ and the asterisks $\left({ }^{*}\right)$ represent statistical significance in comparison with free DOX assay for $\mathrm{p}<0.05$ determined by Student's $\mathrm{t}$-test.

Figure 5.9.

Cell viability assays of cardiomyocytes (iPSCs) and breast cancer $(\mathrm{SkBr} 3)$ spheroids obtained after 5 days of chemotherapeutic treatment (free or encapsulated DOX) in dual-organ-on-a-chip bioreactors. Live cells stained with green Calcein-AM dye and red cells showing DOX uptake.

Figure 5.10 .132

Schematic represents of the feedback loop of dual-organ platform (comprising breast cancer and cardiac organoids) integrated with multiplexed electrochemical analysis for the chemotherapy study effect of doxorubicin using the free or encapsulated drug. 


\section{List of Tables}

Table 3.1.

Experimental parameters used to perform the haemodynamic study of RBCs, as natural biophysical markers to evaluate the MNPs biocompatibility by assessing their DIs

Table 3.2.

Physicochemical properties of the synthesized nanoparticles: diameter determined by TEM ( $\left.\mathrm{d}_{\text {TEM }}\right)$, crystallite size calculated from XRD $\left(\mathrm{d}_{\mathrm{hkl}}\right)$, lattice constant and crystalline core phase obtained by XRD analysis

Table 3.3.

Magnetic properties of the $\mathrm{Fe}_{3} \mathrm{O}_{4}$ nanoparticles synthesized by co-precipitation at different synthesis temperatures $\left(30{ }^{\circ} \mathrm{C}\right.$ and $\left.55{ }^{\circ} \mathrm{C}\right)$ : saturation magnetization $\left(M_{s}\right)$, coercivity $\left(H_{c}\right)$, saturation remanence $\left(M_{r}\right)$, SAR and ILP

Table 3.4.

Longitudinal $\left(r_{1}\right)$ and transverse $\left(r_{2}\right)$ relaxivity of MAG30@Al and MAG50@Al. The corresponding coefficient of the linear fit $\left(R_{2}\right)$ and relaxivity ratio $\left(r_{2} / r_{1}\right)$ are also shown

Table 3.5.

Flow characteristics obtained for the four sections of the microchannel in the haemodynamic study

Table 3.6.

Calibration experimental tests and the parameters considered in the infrared camera

Table 3.7.

Experimental assays and the parameters considered to assess the heat generated by the different MNPs under an AMF

Table 3.8. 70

Physicochemical properties of the synthesized nanoparticles: crystallite size calculated from XRD $\left(d_{\mathrm{XRD}}\right)$, lattice constant and crystalline core phase obtained by XRD analysis

Table 3.9. 71

Temperature results obtained with TC (Thermocouple temperature), FLIR (Infrared camera pointer temperature) and MAT (mean temperature of the region of interest obtained in MATLAB) during calibration tests

Table 4.1.

Effect of the amount of the precursor resorcinol, formaldehyde and TEOS, over the shell and void thickness of GYSMNPs 
Table 4.2.

Textural properties of the synthesized materials: specific surface area $\left(\mathrm{S}_{\mathrm{BET}}\right)$, non-microporous surface area $\left(S_{\text {meso }}\right)$, micropore volume $\left(V_{\text {micro }}\right)$, total pore volume $\left(V_{\text {total }}\right)$, ratio between micropore and total pore volume $\left(\mathrm{V}_{\text {micro }} / \mathrm{V}_{\text {total }}\right)$ and average pore diameter $\left(\mathrm{d}_{\text {average }}\right)$

Table 4.3

88

Magnetic properties of the uncoated $\mathrm{Fe}_{3} \mathrm{O}_{4}$ and as-synthesized GYSMNPs: saturation magnetization $\left(M_{s}\right)$, coercivity $\left(H_{c}\right)$ and saturation remanence $\left(M_{r}\right)$

Table 4.4.

Colloidal and dimensional properties of GYSMNP@PF127: void thickness, hydrodynamic diameter $\left(D_{H}\right)$, polydispersion $(P D I)$ and zeta potential

Table 4.5. 100

Drug loading on GYSMNP@PF127 at different initial DOX concentrations (50 - $500 \mu \mathrm{g} \mathrm{mL}^{-1}$ ) in phosphate buffer $\mathrm{pH}$ 7.4. Standard deviation of triplicate drug loading tests $(n=3)$

Table 4.6. 103

Drug release kinetic analysis of GYSMNP@PF127-DOX

Table 5.1.

Selected cardiac and breast cancer biomarkers and their clinical cut-off levels

Table 5.2. 130

Anticancer drug induced experiments using dual-organ-on-a-chip microfluidic bioreactor 


\section{Glossary}
AMF
Alternating magnetic field
C
Specific heat capacity of the medium
CE
Counter electrode
CKMB
Human creatine kinase
CVD
Chemical vapor deposition
DI
Deformation index
DLS
Dynamic light scattering
DOX
Doxorubicin
$d_{\text {pore }}$
Average pore diameter
$\mathrm{dXRD}_{\mathrm{X}}$
Crystallite size calculated from XRD
EC
Electrochemical
EDTA
Ethylenediaminetetraacetic acid
EIS
Electrochemical impedance spectroscopy
ELISA
Enzyme-linked immunosorbent assay
EPR
Enhanced permeability and retention
$f$
Frequency
$\mathrm{Fe}_{3} \mathrm{O}_{4}$
Magnetite
FTIR
Fourier-transform infrared spectroscopy
GbMNPs
Graphene-based magnetic nanoparticles
GelMA
Gelation methacryloyl hydrogel
GI50
Concentration that inhibits the growth of cells by $50 \%$
GO
Graphene oxide
GYSMNPS
Graphene-based yolk-shell magnetic nanoparticles
$\mathrm{H}$
Amplitude
$\mathrm{H}_{\mathrm{c}}$
Coercivity
HepG2
Human liver cancer cell line
HER2
Human epidermal growth factor Receptor 2
HR
Haemolysis ratio
HR-STEM
High-resolution scanning transmission electron microscopy
ICP-OES
Inductively coupled plasma - optical emission spectrometer
ILP
Intrinsic loss power
iPSCs
Healthy induced pluripotent stem cardiomyocytes
K
Anisotropy constant 
$k_{\mathrm{B}}$

LoC

MCNs

MDDS

MEMS

MFH

$\mathrm{M}-\mathrm{H}$

MNPs

Mr

MRI

$M_{s}$

NIR

NPs

PCR

PDI

PDMS

PF127

$\mathrm{pH}_{\mathrm{PZC}}$

PLP2

PMMA

PoC

PP

PSS

RBCs

$\mathbf{R}_{\mathrm{ct}}$

RE

$\operatorname{Re}_{p}$

rGO

SAM

SAR

$S_{\text {BET }}$

SEM

SkBr3

$S_{\text {meso }}$

SPIONs

SQUID-VSM

$T$
Boltzmann constant

Lab-on-a-chip

Magnetic carbon nanostructures

Magnetic drug delivery systems

Micro-electrochemical systems

Magnetic fluid hyperthermia

Hysteresis loops

Magnetic nanoparticles

Remanence magnetisation

Magnetic resonance imaging

Saturation magnetisation

Absorbing near infrared

Nanoparticles

Polymerase chain reaction

Polydispersion index

Polydimethylsiloxane

Copolymer pluronic F-127

$\mathrm{pH}$ at the point of zero charge

Porcine liver primary cells

Polymethylmethacrylate

Point-of-care

Polypropylene

Physiological salt solution

Red blood cells

Electron-transfer resistance

Reference electrode

Particle Reynolds number

Reduced graphene oxide

Self-assembly monolayer

Specific absorption rate

Specific surface area

Scanning electron microscopy

Breast adenocarcinoma cell lines

Non-microporous specific surface area

Superparamagnetic iron oxide nanoparticles

Superconducting quantum interference device

Temperature 
$T_{1}$

$T_{2}$

TEM

TG

TGA

V

$V_{H}$

$\mathrm{V}_{\text {micro }}$

$V_{\text {total }}$

WE

XRD

$\gamma-\mathrm{Fe}_{2} \mathrm{O}_{3}$

YSNs

ZFC-FC

ZP

$\eta$

$\mu$ TAS

$\tau$

$\tau_{B}$

$\tau_{\mathrm{N}}$
Longitudinal relaxation

Transverse relaxation

Transmission electron microscopy

Thermogravimetric analysis

Thermogravimetric analysis

Volume of the MNP

Hydrodynamic volume of the particle

Micropore volume

Total pore volume

Working electrode

$X$-ray diffraction

Maghemite

Yolk-shell nanostructures

Zero-field-cooled and field-cooled

Zeta potential

Viscosity of the liquid carrier

Micro total analysis system

Relaxation time

Brownian relaxation

Néel relaxation 



\section{CHAPTER I Introduction}

1.1 Contextualization

1.2 Objectives and research plan

1.3 Contributions

1.4 Thesis structure 



\section{Introduction}

\subsection{Contextualization}

According to the last statistic data published by Eurostat on November of 2014, cancer represents the second most common cause of death in the European Union countries. Just in 2011, across the EU28, cancer was responsible for the death of 1.281 million people, representing more than a quarter of all deaths, and an increase of $6.3 \%$ since 2002 [1]. This high rate of mortality is a clear indicator of the clinical urgency to develop new medicines, treatments and detection strategies, as well as an opportunity to improve the existing treatment techniques, to minimize their deleterious side effects and to increase the patient survival rates. Arising from this clinical need and the emerging of nanotechnology, magnetic nanoparticles (MNPs) are gaining increasing attention in the scientific community, especially for biomedical applications, due to their remarkable properties acquired at the nanoscale level. Among them, superparamagnetism, high surface-to-volume ratio, high field irreversibility, high saturation field, extra anisotropy contributions, biocompatibility and low toxicity, are some of those properties that have highlighted the potentiality of MNPs [2-5].

Based on these outstanding properties, magnetic fluid hyperthermia (MFH) is considered a promising therapeutic technique for the treatment of cancer since it implements the remarkable nanoscale physicochemical properties of MNPs to generate heat under an alternating magnetic field (AMF). These nanoheaters can be designed to preserve healthy cells while destroying selectively tumoural ones, inherently more sensitive to mildtemperatures changes of 5 to $7{ }^{\circ} \mathrm{C}$ above the body temperature [6, 7]. In fact, MFH has several advantages over the common used therapeutic strategies, such as the possibility to chemically functionalize the nanoheaters with specific targeting molecules, such as aptamers and antibodies. In addition, MNPs can be used as a non-invasive imaging agent in magnetic resonance imaging (MRI) and tumour therapy - offering the possibility to develop an image-guided therapy system - the so-called theranostics [8]. Although hyperthermia can cause direct damage to cancer cells when applied as single therapy, this technique shows promising results when applied as an adjuvant to other treatments (such as radio-therapy, chemotherapy, gene therapy and immunotherapy), with minimal or no injury at all of the normal tissues $[6,8]$.

Nevertheless, to get the desired therapeutic effect, MFH needs to fulfil several requirements that should be suitably balanced. For that, MNPs should be designed small enough (i) to be able to cross the blood barrier and to penetrate into the tumour; (ii) to escape from the 
mononuclear phagocyte system, providing an increase in the blood circulation half-life; (iii) to have low sedimentation rates (i.e., high stability in suspension); and (iv) to show a good tissular diffusion [2, 4]. Additionally, after the removal of the external magnetic field, MNPs should lose the magnetization (with negligible remanence and coercivity), in order to prevent their aggregation or the formation of clots in the blood circulation system [9-11]. Thus, MNPs must be in the single domain particle size range, showing superparamagnetic behaviour (below 25-30 nm for magnetite $[12,13]$ ) at room temperature. But, the reduction of the MNPs size is directly related to the reduction of the saturation magnetization $\left(M_{s}\right)$ and, therefore, to the decrease of their heating efficiency $[9,10,14]$. This fact is especially important for MFH applications since the reduction of $M_{s}$ requires the maximization of the external magnetic field (amplitude, applied magnetic field and frequency) to values sometimes higher than the physiological safety limit $\left(\mathrm{Hf}<4.85 \times 10^{8} \mathrm{Am}^{-1} \mathrm{~s}^{-1}\right.$, also known as the Brezovich criterion [15]) and/or the application of high MNP dosages that may increase the possibility of toxicity. In this context, a major concern in the synthesis of nanoparticles for biomedical applications is the nanosize control that can be manipulated by changing the synthesis parameters, such as $\mathrm{pH}$, temperature, ion species ratio, ionic strength, among others $[16,17]$. For that reason, many synthesis routes have been developed to produce MNPs with specific magnetic properties, considering several preparation methods, including co-precipitation, sol-gel, hydrothermal, electrochemical synthesis, thermal decomposition, laser pyrolysis and sonolysis [2, 10, 16, 17]. Regarding the synthesis methods, some aspects should be considered. For instance, it is highly recommended that the developed procedure allows the production of a set of MNPs with the following characteristics: (i) reproducibility; (ii) lower polydispersion of single-domain superparamagnetic nanoparticles; (iii) high $M_{s}$ and heating power; (iv) biocompatibility and non-toxicity; and (v) high colloidal stability in physiological buffers with lower dipolar interactions (i.e., physiological salt solution, phosphate-buffered saline, among others). For the two last items (iv and v), the coating of the MNP plays a very important role.

Overall, crystalline magnetic particles are foreign species for the human body, which can be rapidly recognized and eliminated by the mononuclear phagocyte system. Thus, it is important to create an appropriate coating to enhance their biocompatibility, biodistribution and escape from the mononuclear phagocyte system [18].

Graphene, the two-dimensional carbon allotrope consisting of a single atomic layer honeycomb network of $s p^{2}$-hybridization [19], has attracted a growing interest of researchers since its isolation in 2004 by A. Geim and K. Novoselov (U. Manchester) - work that was recognized in 2010 with the Nobel Prize in Physics. Their unique physicochemical properties, such as mechanical and thermal stability, supercondutivity, inertness and biocompatibility 
characteristics, as well as the possibility to easily modify their surface with targeting ligands or active agents, made graphene the perfect candidate to be applied as coating material on MNPs for biomedical applications [20]. In fact, in literature, several biomedical applications are considering the combination of carbon nanostructures with magnetic nanomaterials to produce hybrid nanomaterials, the so-called magnetic carbon nanostructures (MCNs) [2023]. This approach has proven to take advantage of the synergism achieved at the nanoscale, namely the stability and biocompatibility properties of carbon structures with the superparamagnetism of the magnetic materials [21, 23]. These ingredients can represent the successful formula to boost the application of MNPs, as the so expected theranostic technique. Nevertheless, to enable the success in these biomedical applications, three important scientific aspects have to be taken into account: (1) synthesis; (2) advanced characterization (physical, chemical, structural, toxicological and magnetic), and (3) biological testing/implementation.

In fact, and in spite of all the progress achieved in the last decade, the translation of these multifunctional therapeutic nanosystems for clinical applications has been slow, especially due to the lack of robust preclinical tissue culture platforms able to mimic the in vivo conditions found in the human body and to predict the performance of the developed nanomaterials [24]. Indeed, it should be noticed that MNPs designed for theranostic applications have been developed to be applied directly into the bloodstream. So, the understanding of these complex cell-nanoparticle interactions is of utmost importance, especially for the development of multifunctional nanoparticles as drug delivery nanocarriers. Currently, toxicological screening processes are performed in in vitro culture systems, by seeding cells within multi-well dishes. Nevertheless, and although their merits, this methodology is not always able to predict the function and effect of drugs in vivo, resulting in a large number of failed drug candidates in animal experiences and clinical trials $[25,26]$. The main shortcoming of these in vitro cellular models, especially for the evaluation of new nanomaterials, is the inability to mimic the complex three-dimensional (3D) in vivo microenvironment, wherein the cells and extracellular matrix (ECM) exist in well-organized architectures [26]. Microfluidic devices, which can be defined as the set of technologies handling and processing small fluid volumes (e.g., $\mu \mathrm{L}, \mathrm{nL}$ and $\mathrm{pL}$ ) through microchannel geometries, with dimension of tens to hundreds of micrometres embedded in a chip [27-30], have several advantages to assess the biophysical properties of cells when compared to conventional techniques. For instance, this technology allows the use of small sample volumes, integration capability of electronic systems, biocompatibility environment for cell studies, accuracy and fast response [31]. In addition, in the recent decades, a significant advance of microfluidic technologies has allowed the design of microchannel 
Contextualization

geometries in the sizes of small vascular capillaries, which can mimic the micro-rheological properties of the blood cells in in vitro microvascular environment. Furthermore, the advances in computers, optics and digital image processing techniques [32], allow the assessment of haemodynamic phenomena recorded in those in vitro microvascular environments. More recently, a new concept of biomicrofluidic devices, called as organ-ona-chip platforms, has been developed to mimic complex human organ functions at the microscale level. These integrated microfluidic networks, with 3D tissue engineered models, have shown the ability to reduce the discrepancies between the preclinical and clinical trials [26]. Moreover, these 3D microfluidic platforms have several advantages over the in vivo animal models, such as lower costs and less time-consuming, end of animal ethical concerns, visualization of the theranostic agents in the target tissues and accuracy to predict human responses [33].

In this sense, the present Ph.D. thesis aims to join these interdisciplinary approaches, envisaging new insights related to many scientific aspects of the utilization of MNPs in biomedical applications, namely mechanistic and toxicological phenomena, which are essential to boost the spread of multifunctional nanoparticles as the next generation of therapeutic bio-agents in medicine. 


\subsection{Objectives and research plan}

The main objective of the present Ph.D. thesis is the development of novel microfluidic devices capable to mimic the in vivo physiological conditions to assess the mechanistic and toxicological phenomena of MCNs designed as multifunctional nanoplatforms for the treatment of cancer. Hence, several specific goals have to be sequentially accomplished, namely:

(i) Synthesis, characterization and optimization of superparamagnetic nanoparticles as magnetic core of MCNs (i.e., superparamagnetic metal cores coated with carbon nanostructures) designed as theranostic nanosystems;

(ii) Development, characterization and optimization of multifunctional MCNs designed for combined treatment of cancer, namely as nanoheaters in magnetic hyperthermia and nanocarriers for drug delivery applications;

(iii) design and fabrication of polydimethylsiloxane (PDMS) novel microfluidic devices for haematological studies, as well as advanced 3D organ-on-a-chip platforms capable to perform drug screening tests of the developed nanomaterials;

(iv) performance of in vitro experiments for the validation of developed nanomaterials as suitable nanosystems for nanomedicine, especially focused in the assessment of the flow behaviour with the blood cells, cell-nanoparticles interactions (i.e., healthy and cancer tissues) and biocompatibility.

To achieve these goals, this Ph.D. study is divided in three main experimental tasks, namely:

1) production, characterization (i.e., physicochemical, structural, toxicological and magnetical) and optimization of MCNs;

2) fabrication and preliminary haemodynamic evaluation of MNPs under microfluidic platforms;

3) evaluation of the MCNs (i.e., biocompatibility, cell-nanoparticle interactions and drug screening) as suitable nanocarriers for the treatment of cancer, by using a novel 3D multiorgan-on-a-chip platform integrated with electrochemical biosensors. 


\subsection{Contributions}

For the successful accomplishment of the multidisciplinary tasks considered for this Ph.D. thesis, four important collaborations were strengthened at different stages in the frame of this Ph.D. project:
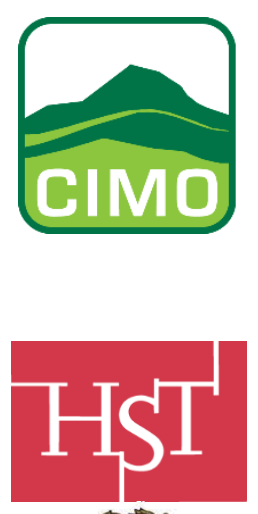

INL - International Iberian Nanotechnology Laboratory (Braga, Portugal), by the Diagnostics tools and Methods group headed by Doctor Manuel Bañobre-Lopez, which had contributed for the physicochemical and magnetic characterization of the MNPs.

CeRiCol - Centro Ricerche Colorobbia Consulting (Vinci, Italy) headed by Doctor Giovanni Baldi that collaborated with hyperthermia and drug delivery studies.

CIMO - Centro de Investigação da Montanha (Bragança, Portugal) headed by Doctor Isabel Ferreira, collaborating with 2D-static cytotoxicological tests of MNPs on healthy and tumour cell lines.

Harvard-MIT Division of Health Sciences and Technology (Cambridge, USA) on the Biomaterials Innovation Research Center headed by Professor Ali Khademhosseini and supervised by Doctor Su-Ryon Shin, which allowed the drug screening of the developed MCNs on novel 3D multiorgan-on-a-chip platforms integrated with electrochemical biosensors. 


\subsection{Thesis structure}

The Ph.D. thesis is structured in six chapters and one annex as shown in Figure 1.1, and described as follows:

1. Introduction. In the present chapter a broad perspective and contextualization of the problematic was addressed. Both objectives and research plan were summarized, and the thesis structure is herein described.

2. Theoretical concepts. Fundamental concepts related to the multidisciplinary topics approached in the thesis are discussed.

3. Superparamagnetic nanoparticles for theranostic applications. The development and optimization of superparamagnetic nanoparticles suitable for the diagnosis and therapy of cancer (theranostic) are described. A novel microfluidic approach based on the assessment of the deformation index of blood cells is implemented to determine the effect of nanoparticles on the bloodstream. Additionally, a thermal infrared image processing is proposed to screen the heating efficiency of magnetic nanoparticles during hyperthermia.

4. Graphene-based magnetic nanoparticles for the treatment of cancer. The development and application of multifunctional graphene-based magnetic nanoparticles for combined hyperthermia and dual stimuli-responsive drug delivery are described.

5. Organ-on-a-chip platform for bio-toxicity evaluation of graphene-based magnetic nanoparticles. In this chapter, a 3D multi-organ model combined with electrochemical biosensing analysis is described for the evaluation of the developed nanoparticles as efficient drug nanocarriers.

6. Conclusions and future perspectives. The main conclusions of the Ph.D. studies are summarized in this chapter. Also, a personal perspective for future works and direction of the nanomedicine field is given.

7. Appendixes. Supplementary information regarding the further understanding of some multidisciplinary topics approached in Chapter II, are detailed in this annex. Additionally, a list of publications, scientific meetings, grants and awards that resulted from the Ph.D. studies is shown. 


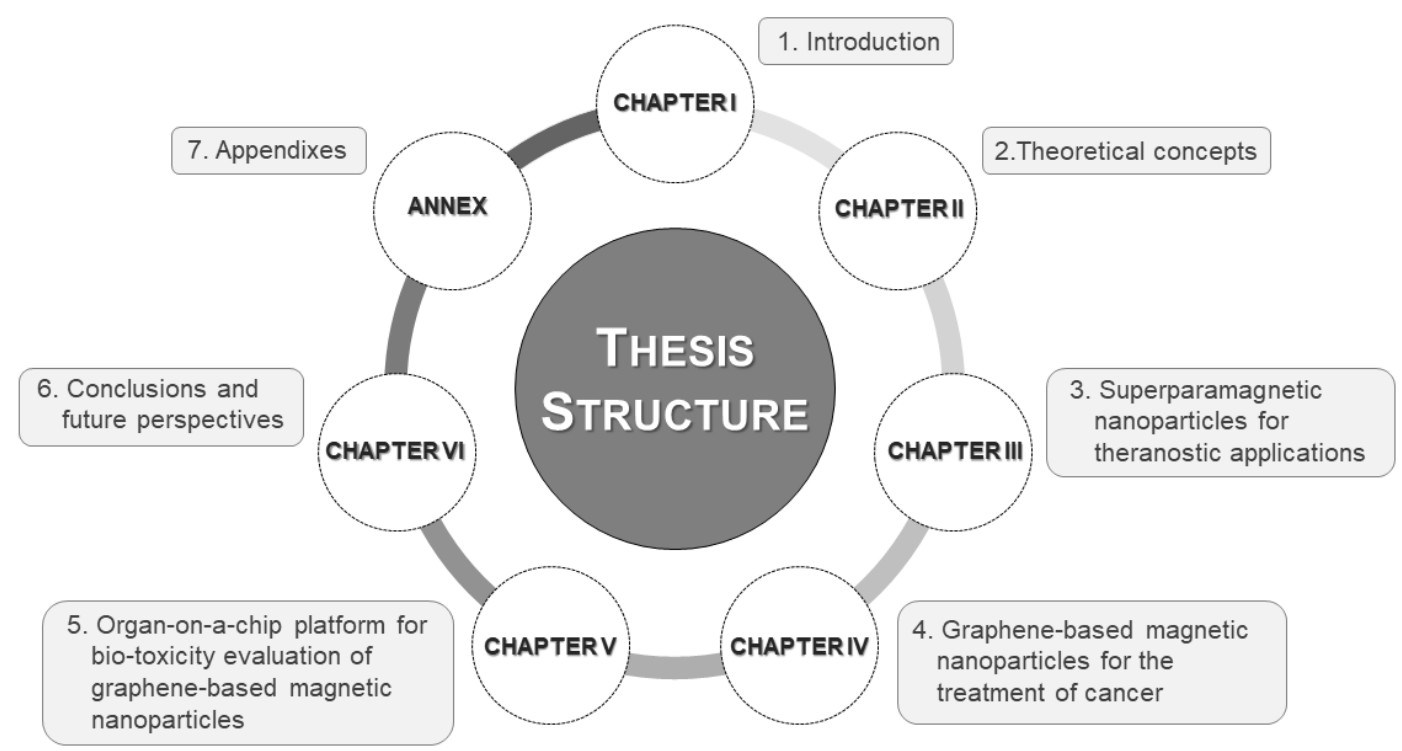

Figure 1.1. Diagram of the six chapters and annex that comprise the Ph.D. thesis. 


\section{References}

[1] Eurostat, News releases - 1 in 4 deaths caused by cancer in the EU28 http: / /ec.europa.eu/eurostat/documents/2995521/6131615/3-25112014-BP-

EN/aab2c2d3-aed9-430a-a561-e188b8ef49d8, Accessed 03/12/2017

[2] M. Mahmoudi, S. Sant, B. Wang, S. Laurent and T. Sen, Advanced Drug Delivery Reviews, 2011, 63, 24-46.

[3] A. Hervault and N. T. K. Thanh, Nanoscale, 2014, 6, 11553-11573.

[4] J. Rivas, M. Bañobre-López, Y. Piñeiro-Redondo, B. Rivas and M. A. López-Quintela, Journal of Magnetism and Magnetic Materials, 2012, 324, 3499-3502.

[5] Y. V. Kolen'ko, M. Bañobre-López, C. Rodríguez-Abreu, E. Carbó-Argibay, A. Sailsman, Y. Piñeiro-Redondo, M. F. Cerqueira, D. Y. Petrovykh, K. Kovnir, O. I. Lebedev and J. Rivas, The Journal of Physical Chemistry C, 2014, 118, 8691-8701.

[6] A. Chichet, J. Skowronek, M. Kubaszewska and M. Kanikowski, Reports of Practical Oncology and Radiotherapy, 2007, 12, 267-275.

[7] A. Jordan, R. Scholz, P. Wust, H. Fähling and F. Roland, Journal of Magnetism and Magnetic Materials, 1999, 201, 413-419.

[8] A. Ito, M. Shinkai, H. Honda and T. Kobayashi, Journal of Bioscience and Bioengineering, 2005, 100, 1-11.

[9] M. Bañobre-López, A. Teijeiro and J. Rivas, Reports of Practical Oncology and Radiotherapy, 2013, 18, 397-400.

[10] I. Sharifi, H. Shokrollahi and S. Amiri, Journal of Magnetism and Magnetic Materials, 2012, 324, 903-915.

[11] I. Obaidat, B. Issa and Y. Haik, Nanomaterials, 2015, 5, 63.

[12] A. K. Gupta and M. Gupta, Biomaterials, 2005, 26, 3995-4021.

[13] V. Prigiobbe, S. Ko, C. Huh and S. L. Bryant, Journal of Colloid and Interface Science, 2015, 447, 58-67.

[14] A. E. Deatsch and B. A. Evans, Journal of Magnetism and Magnetic Materials, 2014, 354, 163-172.

[15] I. A. Brazovich, Medical Physics Monograph, 1988, 16, 82-111.

[16] J. Baumgartner, L. Bertinetti, M. Widdrat, A. M. Hirt and D. Faivre, PLoS ONE, 2013, 8, e57070.

[17] R. Hao, R. Xing, Z. Xu, Y. Hou, S. Gao and S. Sun, Advanced Materials, 2010, 22, 27292742.

[18] COST, European Cooperation in Science and Technology, Brussels, 2014.

[19] G. Bharath, R. Madhu, S.-M. Chen, V. Veeramani, D. Mangalaraj and N. Ponpandian, Journal of Materials Chemistry A, 2015, 3, 15529-15539.

[20] Y. Yang, A. M. Asiri, Z. Tang, D. Du and Y. Lin, Materials Today, 2013, 16, 365-373.

[21] S. Boncel, A. P. Herman and K. Z. Walczak, Journal of Materials Chemistry, 2012, 22, 31-37.

[22] S. Y. Qin, J. Feng, L. Rong, H. Z. Jia, S. Chen, X. J. Liu, G. F. Luo, R. X. Zhuo and X. Z. Zhang, Small, 2014, 10, 599-608. 
[23] Y. Xu, M. Mahmood, A. Fejleh, Z. Li, F. Watanabe, S. Trigwell, R. B. Little, V. P. Kunets, E. Dervishi, A. R. Biris, G. J. Salamo and A. S. Biris, International Journal of Nanomedicine, 2010, 5, 167-176.

[24] N. S. Bhise, J. Ribas, V. Manoharan, Y. S. Zhang, A. Polini, S. Massa, M. R. Dokmeci and A. Khademhosseini, Journal of Controlled Release, 2014, 190, 82-93.

[25] Y. S. Zhang, J. Aleman, S. R. Shin, T. Kilic, D. Kim, S. A. Mousavi Shaegh, S. Massa, R. Riahi, S. Chae, N. Hu, H. Avci, W. Zhang, A. Silvestri, A. Sanati Nezhad, A. Manbohi, F. De Ferrari, A. Polini, G. Calzone, N. Shaikh, P. Alerasool, E. Budina, J. Kang, N. Bhise, J. Ribas, A. Pourmand, A. Skardal, T. Shupe, C. E. Bishop, M. R. Dokmeci, A. Atala and A. Khademhosseini, Proceedings of the National Academy of Sciences, 2017, 114, E2293-E2302.

[26] Y. S. Zhang, Y.-N. Zhang and W. Zhang, Drug Discovery Today, 2017, 22, 1392-1399.

[27] S. Halldorsson, E. Lucumi, R. Gómez-Sjöberg and R. M. T. Fleming, Biosensors and Bioelectronics, 2015, 63, 218-231.

[28] R. Monošík and L. Angnes, Microchemical Journal, 2015, 119, 159-168.

[29] S. K. Sia and G. M. Whitesides, Electrophoresis, 2003, 24, 3563-3576.

[30] G. M. Whitesides, Nature, 2006, 442, 368-373.

[31] Y. Zheng, J. Nguyen, Y. Wei and Y. Sun, Lab on a Chip, 2013, 13, 2464-2483.

[32] R. Lima, S. Wada, S. Tanaka, M. Takeda, T. Ishikawa, K. Tsubota, Y. Imai and T. Yamaguchi, Biomedical microdevices, 2008, 10, 153-167.

[33] J. Tan, A. Thomas and Y. Liu, Soft Matter, 2012, 8, 1934-1946. 


\section{CHAPTER II Theoretical concepts}

2.1 Magnetism and magnetic nanoparticles

2.2 Magnetic carbon nanostructures

2.3 Microfluidic devices 



\section{Theoretical concepts}

\subsection{Magnetism and superparamagnetic nanoparticles}

The modern theories of magnetism in solids are based on the orbital motion of electrons around the nucleus and on the spin motions of electrons. Therefore, electrons produce a spin magnetic moment of the unpaired valence electrons, as well as an orbital magnetic moment $[1,2]$.

This concept means that when a solid is placed under a magnetic field, the magnetic dipoles inside the material are aligned along with the direction of the applied magnetic field, and so, magnetized.

The magnetic induction or magnetic flux density, called by $B$ (in Tesla or T) and generally illustrated as the number of field lines per unit area, can be expressed by Equation (2.1) [3]:

$$
\mathrm{B}=\mu_{0}(\mathrm{H}+\mathrm{M})
$$

where $\mu_{0}$ is the permeability in vacuum $\left(4 \pi \times 10^{-7} \mathrm{H} \mathrm{m}^{-1}\right), H$ is the magnetic field strength or magnetizing force $\left(\mathrm{A} \mathrm{m}^{-1}\right)$ and $M$ is the magnetic moment per volume $\left(\mathrm{A} \mathrm{m}^{-1}\right)$.

Figure 2.1 shows the redirection of the magnetic field lines caused by the great permeability occurring when a magnetic field is intercepted by a magnetic material, e.g., soft iron.

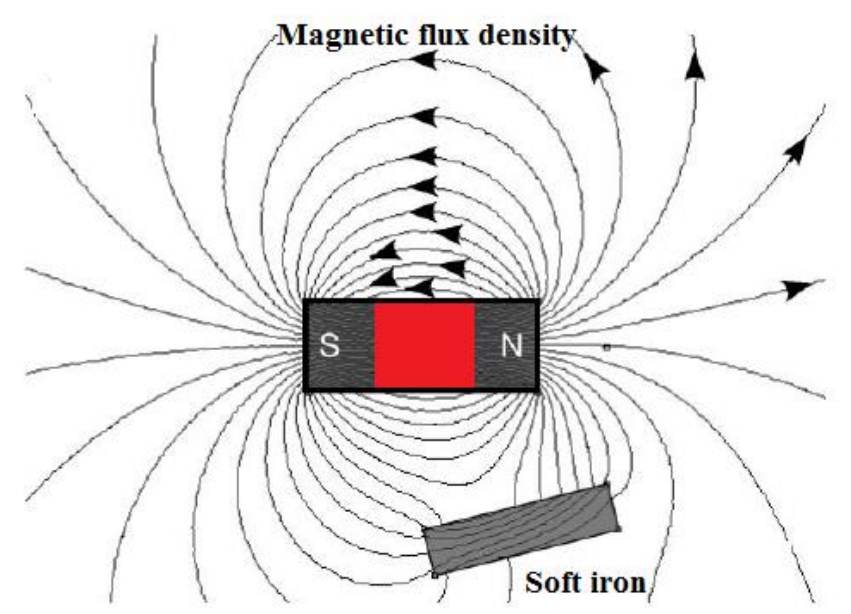

Figure 2.1. Permeability of the magnetic flux density caused by a magnetic material, for instance soft iron. Reprinted from [4]. Copyright @ 2006, with permission from Royal Society of Chemistry. 
According to the volumetric magnetic susceptibility, represented by $\chi$, the magnetic materials can be classified as:

- Diamagnetic materials $(\chi<0)$ : slightly repelled from magnetic fields, usually called as non-magnetic materials, such as gold, water and copper [1, 4];

- Paramagnetic materials $(\chi>0)$ : slightly attracted to magnetic fields, but they do not retain the magnetic properties when the external fields are removed. This class includes oxygen, platinum and manganese (II) salts [1, 4];

- Ferromagnetic materials $(\chi>>0)$ : exhibit a strong attraction to magnetic fields due to the alignment of the spin magnetic moments and retain their magnetic properties even after removal of the external field. Metals, such as iron, cobalt, nickel and many alloys containing these elements, are some examples [4];

- Antiferromagnetic materials $(\chi>0)$ : exhibit regular pattern of parallel and antiparallel atoms aligned when a magnetic field is applied, resulting in a small magnetization that increases further with temperature, but evanish when the magnetic field is removed. Examples of this type of materials are chromium and nickel oxide $[1,5]$;

- Ferrimagnetic materials $(\chi>>0)$ : are identical to antiferromagnetic materials but the regular pattern of parallel and antiparallel atoms alignment has a higher value of magnitude. The most common example is magnetite (or ferrous ferrite) $[1,5]$.

Additionally, there is a special case of magnetism, the superparamagnetism.

Superparamagnetism occurs in ferromagnetic or ferrimagnetic nanoparticles, generally below $100 \mathrm{~nm}$, with a single magnetic domain, short relaxation time and no magnetic memory. Once the external field is removed, their magnetic moment is quickly reoriented, leading to a loss of energy that heats the surrounding environment. After that, the particles re-disperse and behave like a non-magnetic material $[1,4,6]$.

These special characteristics make them ideal candidates for biomedical applications, such as enhancement contrast agent in magnetic resonance imaging (MRI), drug delivery therapy, cell and tissue targeting and especially, for magnetic hyperthermia therapy [3, 7]. 


\subsubsection{Magnetic hyperthermia, biological principle and heating mechanism}

Hyperthermia, also called thermotherapy, is a technique used for cancer treatment that consists in heating tumour organs or tissues to temperatures between 41 and $45{ }^{\circ} \mathrm{C}[8,9]$, promoting thermal apoptosis of these abnormal cells by exceeding their physiological tolerance [10].

The concept of hyperthermia as a medical practice can be tracked down to many centuries ago, as being present in ancient Egyptian, Greek and Roman civilizations [11]. However, only recently, on the 1970s, hyperthermia was taken more seriously as a treatment for cancer with controlled clinical trials. During that time, it was discovered that tumour cells have greater sensitivity to mild temperatures compared to healthy cells [12].

On a general biological perspective, this different response has been attributed to the disorganized and compact vascular structure, anaerobic metabolism and nutrient depletion of the abnormal tumour tissues, when compared to healthy ones [13]. In contrast, healthy cells are capable to survive, even when exposed to temperatures around $46^{\circ} \mathrm{C}$, mainly due to the capability to dissipate heat through their normal vascular system and to maintain a normal physiological temperature $[10,13]$.

While the exact biomolecular mechanism behind hyperthermia to kill tumour cells is not yet fully understood, it is known that local heat generated by the hyperthermia treatment has the cancer cells proteins as predominant target, especially, regulatory proteins involved in cell growth and differentiation, and proteins involved in the expression of certain receptor molecules, such as the signal transduction pathways. Thereby, hyperthermia seems to act on the cellular membrane destabilization by denaturing intracellular proteins, which in turn has great influence in cytoskeleton, enzymes, signal transduction, macromolecular synthesis and cell nucleus, by interfering with DNA repair system and leading to apoptosis [9, 14]. Additionally, other effects have been also observed at the tissue level, namely changes in the microvasculature, which result in alterations on blood flow, tumour oxygenation and energy supply $[9,14]$. These later phenomena have been identified as the cause for the synergistic effect of hyperthermia with chemotherapy during clinical trials $[15,16]$. Thus, the increase of local temperature promoted by thermotherapy allows (i) an increase in drug concentration in the tumour area, (ii) an increase of intracellular drug uptake, and (iii) an enhancement on the DNA damaging [11], when compared to the effect of chemotherapy alone.

Although a wide range of heat sources and treatments based on hyperthermia concept have been developed for clinical applications - including microwave (in the range of wavelengths 
from 433 to $2450 \mathrm{MHz}$ ), radiofrenquency (ranging from $100 \mathrm{KHz}$ to $150 \mathrm{MHz}$ ), laser and ultrasonic wave-based treatments, hot water perfusion, among others [3, 14]; magnetic fluid hyperthermia (MFH) is the one that is considered to have the best potential to selectively target the tumour cells [3].

MFH therapy consists on a colloidal suspension of MNPs that are capable to reach and internalize tumour cells, and thus, generate local heat when subjected to an external alternating magnetic field (AMF). Differently from other hyperthermic therapies, MFH heating effect is based on the conversion of magnetic energy to thermal energy, due to several mechanisms that are intrinsically dependent on the size of the nanomaterial that is used $[11,17]$.

In the case of multi-domain NPs (ferri- or ferromagnetic material), the amount of energy dissipated as heat during magnetisation is mainly due to hysteresis losses $[3,18]$. Thus, when an external AMF is applied, all the magnetic domains tend to align in the direction of the applied field, reaching their saturation magnetisation $\left(M_{\mathrm{s}}\right)$ once all the domains are aligned toward this direction. After the external AMF is removed, the magnetisation does not revert to zero, and it is called as remanence magnetisation $\left(M_{r}\right)$. The precise intensity needed to be applied to reduce the magnetisation back to zero is called coercivity $\left(H_{c}\right)$. These parameters can be measured from the hysteresis loops $(M-H)$ of the material (Figure 2.2(a)), and the hysteresis losses measured by integrating the area of the hysteresis loop $[3,11,18$, 19].

(a)

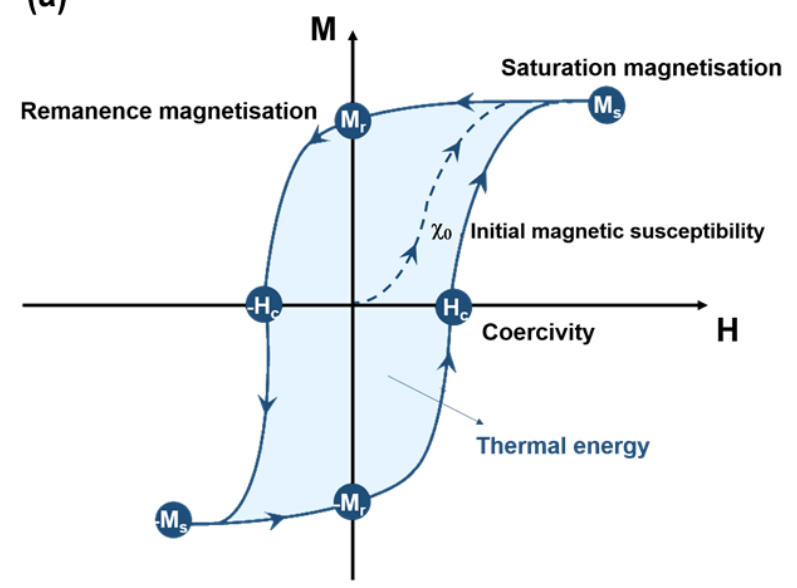

(b)

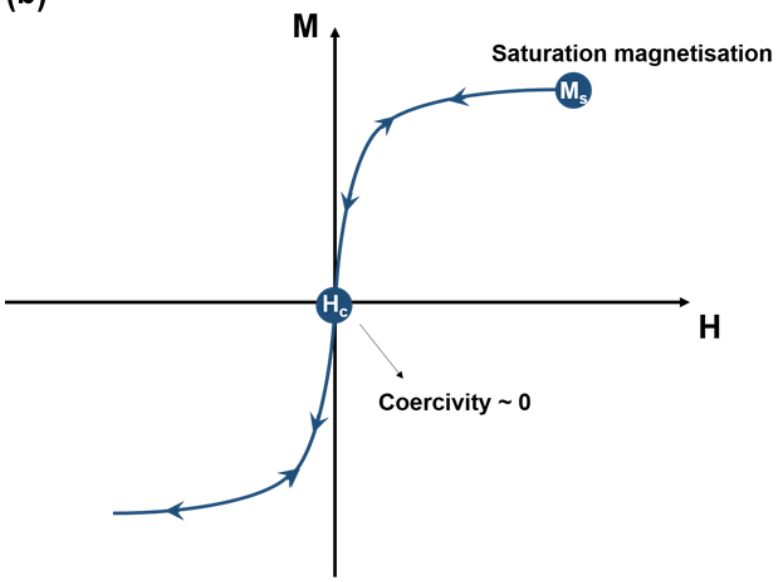

Figure 2.2. Schematic representation of a typical hysteresis loop $(M-H)$ of: (a) multi-domain ferromagnetic material and (b) single-domain superparamagnetic material.

However, when MNPs are very small, below a critical diameter (typically about 10-100 nm for a wide range of materials [18]), the material is not able to support more than one domain and, thereby, called as single-domain. Single-domain NPs comprise a giant magnetic moment 
composed off all magnetic moments of the atoms that form the NP [11]. In the case of singledomain superparamagnetic NPs, the $\mathrm{M}-\mathrm{H}$ curve does not show hysteresis, and the forward and backward magnetisation curves tend to overlap [18]. As a result, the remanence magnetisation and coercivity are negligible (Figure 2.2(b)), offering advantages for biomedical applications by reducing the risk of particles aggregation after removal of the external AMF.

In this case, the heat dissipation mechanism is mainly caused not by the hysteresis losses, but due to the delay in the relaxation of the magnetic moment, i.e., Néel relaxation (rotation within the particle) and/or Brownian relaxation (rotation of the particle itself), $[3,18]$.

Néel, $\tau_{\mathrm{N}}$, and Brownian, $\tau_{\mathrm{B}}$, magnetic relaxation times are given by the Equations (2.2) and (2.3), respectively [3]:

$$
\begin{gathered}
\tau_{N}=\tau_{0} \exp \left(\frac{K V}{k_{B} T}\right) \\
\tau_{B}=\frac{3 \eta V_{H}}{k_{B} T}
\end{gathered}
$$

where $\tau_{0}=10^{-9} \mathrm{~s}, K$ is the anisotropy constant, $V$ the volume of the MNP, $k_{B}$ the Boltzmann constant, $T$ the temperature, $\eta$ the viscosity of the liquid carrier and $V_{H}$ the hydrodynamic volume of the particle.

Néel relaxation is strongly size-dependent, as smaller NPs require less energy for the rotation of its magnetic moment $[11,18]$. On the other hand, Brownian relaxation, besides sizedependent, is also strongly viscosity-dependent, as NPs can slow down their rotation at high viscose liquid medium [11, 18]. Therefore, for hyperthermia applications, the Néel relaxation mechanism is preferred, since NPs should be internalised on cells with higher viscosities and its ideal free rotation can be reduced on it.

The combination of Equation (2.2) and (2.3) gives the effective relaxation time, $\tau$, of the MNPs, shown as Equation (2.4):

$$
\tau=\frac{\tau_{B} \tau_{N}}{\tau_{B}+\tau_{N}}
$$

The quantification of the power dissipation of MNPs under an AMF, known as specific absorption rate (SAR) and expressed as $\mathrm{W} \mathrm{g}^{-1}$, is given by Equation $(2.5)[3,11,18]$ : 
Magnetism and superparamagnetic nanoparticles

$$
S A R=\frac{C m_{\text {medium }}}{m_{N P S}} \frac{\Delta T}{\Delta t}
$$

where $C$ is the specific heat capacity of the medium, $m_{\text {medium }}$ and $m_{\text {NPs }}$ are the mass of medium and NPs in the dispersion, respectively, and $\Delta T / \Delta t$ is the initial slope of the time-dependent heating curve.

The SAR parameter allows the determination of heating in biological tissues, by estimating the rate at which electromagnetic energy is absorbed by unit mass [3].

It is important to bear in mind that SAR is not only dependent on particle features (i.e., size, size distribution, shape, chemical composition, saturation magnetization), but also on the amplitude, $H$, and frequency, $f$, of the applied magnetic field. Thus, in order to apply hyperthermia in a safer manner, without affecting healthy tissues or produce excessive heating in the patients, it is important to consider an $\mathrm{Hf}$ factor that should not exceed the threshold of $4.85 \times 10^{8} \mathrm{~A} \mathrm{~m}^{-1} \mathrm{~s}^{-1}$, also known as Brezovich criterion [20]. For this purpose, MNPs should be designed with optimized characteristics, including high $M_{\mathrm{s}}$, which allows the maximization of their heating effect without need to exceed this biological threshold or the administration of high dosages of nanoheaters.

Among different nanomaterials, magnetite $\left(\mathrm{Fe}_{3} \mathrm{O}_{4}\right)$ was the first used to obtain a magnetic fluid in 1960 by NASA [18]. Afterwards, other ferrites, $\mathrm{MFe}_{2} \mathrm{O}_{4}(\mathrm{M}=\mathrm{Fe}, \mathrm{Mn}, \mathrm{Ni}, \mathrm{Co}, \mathrm{Zn})$, some alloys as FePt, NiPt and NiPd, as well as pure metals (Fe, $\mathrm{Co}, \mathrm{Ni}$ ), where synthesized and tested for hyperthermia application [7, 12, 18, 21, 22]. Although pure metals have the highest $M_{s}$, these NPs are very toxic and extremely susceptible to oxidation after synthesis [18]. On the other hand, iron oxide NPs, as magnetite $\left(\mathrm{Fe}_{3} \mathrm{O}_{4}\right)$ and maghemite $\left(\gamma-\mathrm{Fe}_{2} \mathrm{O}_{3}\right)$, are less sensitive to oxidation and possess lower toxicity to cells. In addition, these nanomaterials can be simultaneously used as contrast agents for magnetic resonance imaging (MRI), combining therapeutics and diagnosis, the so-called theranostics [11]. However, bare MNPs present in general several limitations, such as their inherent tendency to aggregate and precipitate inside blood vessels, low colloidal stability and low biocompatibility [23]. Thus, to achieve a safer application of these nanomaterials in nanomedicine, it is highly recommended that MNPs are covered by an organic or inorganic biocompatible coating. Among these coatings, the most common are dextran-based coatings [24], polyethythylene glycol [25], chitosan [26], silica [27], gold [28] and carbon-based nanomaterials [27, 29]. 


\subsection{Magnetic carbon nanostructures}

Magnetic carbon-based nanostructures are composite nanomaterials comprising magnetic and carbon structures conjugated in different configurations [30]. Carbon-based materials like graphite, fullerenes, nanotubes and nanoribbons have been used for various applications, such as electronic, optics, catalysis, biomedical engineering, tissue engineering, medical implants, medical devices and sensors, due to their unique chemical and physical properties [31]. Particularly, graphene-based nanomaterials (cf. Figure 2.3) graphene and its derivatives - have accomplished impressive achievements in nanomedicine and biomedical applications thanks to the combination of their unique properties, such as high chemical and thermal stability, high charge carrier mobility, enhanced biocompatibility and large surface area, which are ideal for biofunctionalizations [32, 33].

The graphene-based family is classified based on their number of layers and chemical modification. Among the most widely used graphene-based nanomaterials are the monolayer graphene, bilayer graphene, multilayer graphene, graphene oxide (GO) and reduced graphene oxide (rGO). Each graphene-based member differs from the other in terms of number of layers, surface chemistry, purity, lateral dimensions, defect density and composition [34].

\section{Graphene}

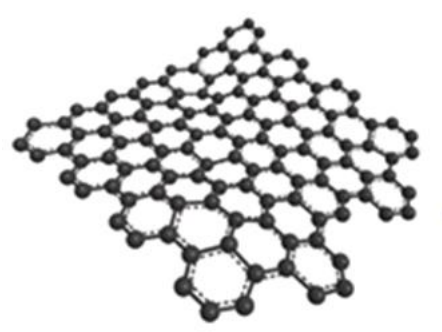

Graphene oxide

(GO)

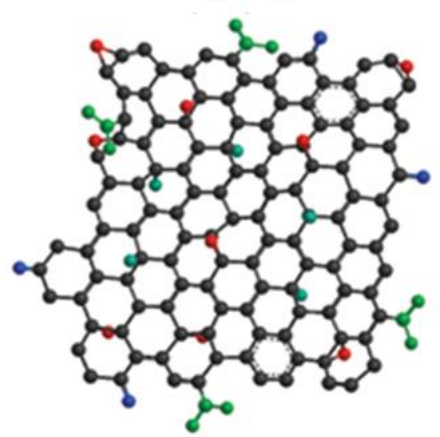

Reduced graphene oxide

(rGO)

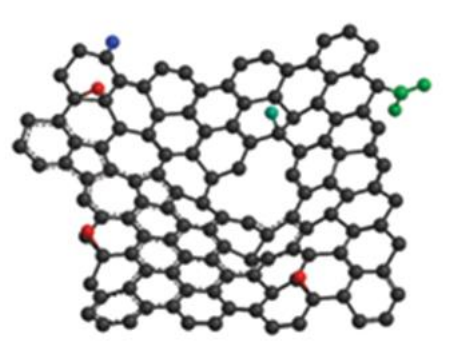

- Carbon

- Epoxy

- Carbonyl

- Hydroxyl

- Carboxyl

Figure 2.3. Schematic chemical structures of graphene-based nanostructures: graphene, graphene oxide and reduced graphene oxide. Reprinted from [35]. Copyright $\odot$ 2016, with permission from Intech.

Graphene, or monolayer graphene, is defined as a single atomic layer of $s p^{2}$ carbon atoms arranged in a honeycomb lattice of nanometer dimensions, and it is the building block of other carbon-based materials, including OD fullerenes, 1D carbon nanotubes or 3D graphite $[36,37]$.

Derived from graphene, GO is a highly oxidized form of chemically modified graphene with a large amount of oxygen-containing groups, essentially epoxy, carbonyl and hydroxyl groups 
$[23,38]$. The peripheral carboxylate group provides $\mathrm{pH}$-dependent negative surface charge and colloidal stability, while epoxide and hydroxyl groups allow weak interactions, hydrogen bonding and other surface interactions [34]. In addition, the free surface $\pi$ electrons promotes $\pi-\pi$ stacking interactions for drug loading and non-covalent functionalization [39]. These features enable the functionalization of a variety of molecules, including metals, chemotherapeutic drugs, DNA and/or RNA, aptamers, fluorescent probes and/or cells [40, 41]. Therefore, GO nanomateriais are ideal platforms for anti-cancer drugs/gene delivery, biosensing, bioimaging, cell culture and tissue engineering [37].

Instead, reduced graphene oxide ( $\mathrm{rGO}$ ) is the reduced form of $\mathrm{GO}$ treated under reducing conditions, such as high-temperature or reducing-chemical treatment. These treatments promote the reduction of oxygen content, introduction of defects in the carbon lattice (due to the liberation of $\left.\mathrm{CO} / \mathrm{CO}_{2}\right)$ and increase of hydrophobicity [23].

Remarkably, the possibility to functionalize graphene-based nanomateriais with polymers, small molecules, magnetic nanoparticles, among others, makes this new class of nanomaterials ideal candidates to enhance or alter its properties accordingly to the requirements of specific applications. Indeed, functionalization is a very important strategy that allows to improve specific properties of magnetic nanocomposites, namely increasing colloidal stability, reducing toxicity, enhancing NP and drug permeability/accumulation into a tumour, among others. In this context, the combination of graphene-based nanostructures with MNPs, i.e., graphene-based magnetic nanoparticles (GbMNPs), allows to overcome some important MNP limitations, such as low biocompatibility and high self-aggregations, and exploit the best properties of both materials.

\subsubsection{Graphene-based magnetic nanoparticles (GbMNPs) in nanomedicine}

Multifunctional nanoparticles can have an important impact in medicine, by allowing the simultaneous detection, treatment and prevention of cancer. Indeed, the design of multifunctional nanoparticles for nanomedicine is one of the most promising and exciting research areas nowadays, since it is expected to revolutionize the medical field in the next few decades [42]. Taking into consideration the synergistic properties of GbMNPs, which can gather the excellent magnetic performance of MNPs with the high specific surface area of graphene-based materials, these magnetic nanocomposites have the potentiality to represent a new clinical platform that fulfil all the requirements for theranostic applications (i.e., simultaneous diagnosis and therapy), as exemplified in Figure 2.4. 


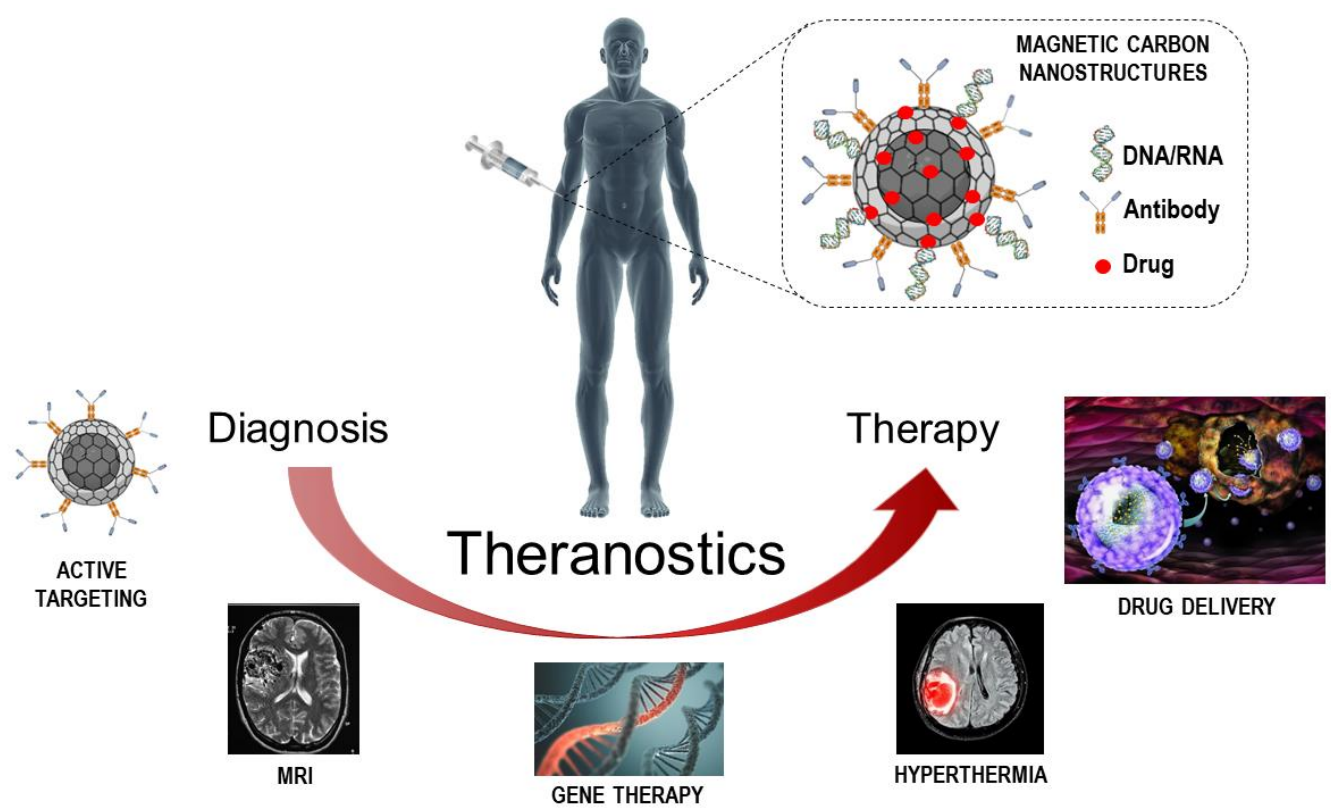

Figure 2.4. Schematic diagram of possible theranostic applications, from diagnosis to therapy, by using magnetic carbon nanostructures, such as graphene-based magnetic nanoparticles.

\subsubsection{Therapeutic applications}

(i) Drug delivery

Chemotherapy is one of the standard treatments used to eliminate tumour tissues. However, selective targeting of tumours is a long standing issue caused by the low specificity of drugs that cause severe side-effects by attacking healthy tissues [31].

Magnetic drug delivery systems (MDDS) allow the transport and delivery of chemotherapeutic drugs into a tumour target site, after being guided by a magnetic field [30]. During the design of MDDS, the release mechanism is an essential question that should be addressed. Ideally, the designed MDDS should retain the therapeutic cargo until reaching the tumour target, and release the drug through specific trigger-response mechanisms, such as environmental factors (e.g. pH variation and enzymatic action) and/or involving properties of the nanocarrier (e.g. temperature-induced release and light irradiation) [30,43]. This strategy holds several advantages over the free drug approach, namely: (i) minimization of the drug amount needed for chemotherapy, (ii) enhancement of drug efficiency, (iii) prevention of severe side-effects by increasing target specificity, and (iv) possibility to combine therapies.

Recently, GbMNPs are being explored as one of the most interesting strategies for bottomup drug delivery system design. The high specific surface area of graphene-based materials allied to $\pi-\pi$ stacking and electrostatic interactions allows to achieve high drug loading of poor soluble drugs without compromising the clinical efficiency [30, 32, 34, 35]. Remarkably, 
magnetic carbon nanostructures also show the intrinsic ability to release the drug cargo under specific trigger-response mechanisms, such as pH-variation [43-45], temperatureinduced release and/or light irradiation [40, 41, 46-48]. These features are especially important since the majority of the designed DMMS - comprising magnetic nanoparticles with non-carbon shell materials, such as polymers, silica or metals - are unable to combine high loading contents of the therapeutic cargo with trigger-response release [42]. Particularly, some recent studies using GO structures as drug nanocarrier have shown the outstanding ability to use this graphene-based nanomaterial as $\mathrm{pH}$ stimuli-responsive triggered by the abnormal acidic $\mathrm{pH}$ values (around 4.5 - 5.5), found in tumour endosome/lysosome microenvironments, but not by physiological pH (around 7.4) [43, 49].

\section{(ii) Magnetic hyperthermia}

Another advantage of GbMNPs is the ability to incorporate superparamagnetic nanoparticles in its structure, which under an external AMF generate heat that leads to the death of cancer cells, by the so-called magnetic hyperthermia (additional details in Section 2.1.1). This possibility opens the door to a combined treatment of cancer, with simultaneous magnetic hyperthermia and controlled drug delivery. In fact, the combination of both treatments has so many advantages that it has been proposed the extension of the concept of hyperthermia, to cover both hyperthermia and magnetically modulated controlled drug delivery through heating triggers [11]. This new treatment concept relies on the possibility to load the drug in stimulus-sensitive shells that protect the cargo until reaching the target site. Then, by slightly increasing the temperature of the magnetic core (e.g. by external AMF), the thermo-sensitive shell suffers structural/conformational changes (e.g. breaking of covalent or non-covalent chemical bonds), which leads to the local release of the drug into the tumour cells [3]. Overall, this last concept seems to use the heat only as a trigger for drug release. However, hyperthermia and chemotherapy can be combined in the same nanosystem, to use hyperthermia as both a treatment and a trigger for controlled drug delivery. In fact, some in vitro and in vivo studies have already demonstrated that the combination of magnetic hyperthermia and chemotherapy has a synergistic effect on the cancer therapy response [50-52]. These same studies also show that even when lower doses of therapeutic drugs are combined with mild-hyperthermia $\left(40-43^{\circ} \mathrm{C}\right)$, the anticancer effects are more effective than when traditional hyperthermia and chemotherapy are applied alone. The enhancement of the chemotherapy effect with the application of hyperthermia is known as thermo-chemosensitisation [11]. Among this advantage in the combination of both cancer therapies, hyperthermia has also demonstrated the potential to reduce the risk of treatment-induced secondary cancer, due to the decrease of oncogenic transformations 
frequently induced by chemotherapy $[11,53]$. One possible explanation to this phenomenon is that the heat produced by hyperthermia inhibits the DNA repair of damaged tumour cells treated with anticancer drugs, thus reducing the expression of chemo-mutations [54].

In this context, for an effective design of smart multifunctional systems, the amount of heat produced by the magnetic core has to be optimized, since the heating mechanism and efficiency strongly depends on the magnetic behaviours of the material. For that, several factors have to be taken into account, namely strength and frequency of the AMF, concentration and depth of the magnetic nanosystem in tissues, as well as their size, shape and composition [3].

GbMNPs have the encouraging advantage to be designed as smart multifunctional nanosystems able to combine magnetic hyperthermia and controlled drug delivery (triggered by changes in the $\mathrm{pH}$ - and/or temperature). Also, it is noteworthy to mention that anticancer thermo-chemotherapy performed with GbMNPs is not just restricted to magnetic induced heating. In fact, graphene-based materials have already shown strong potentiality to generate heat by absorbing near infrared (NIR) light, the so-called phototherapy $[49,55]$.

(iii) Gene therapy

Gene therapy is another therapeutic application that can be combined both with hyperthermia and drug delivery. This therapy has the potentiality to treat genetic disorders, such as cystic fibrosis, Parkinson's disease and cancer. However, it requires efficient and safe gene nanovectors able to protect the genetic content from nuclease degradation, as well as enabling the genomic uptake with high transfection efficiency [34]. Graphene-based materials have been recently explored and described in literature as exceptional vehicles for this application [30, 34, 56], showing promising properties that fit the requirements to the design of non-viral vectors for gene delivery, namely (i) facile and versatile chemical functionalization, (ii) ability to condense genetic material, (iii) protection of nucleic acid from enzymatic degradation, (iv) cellular internalization, and (v) low toxicity [56]. Although these studies have emphasized the potential of graphene-based materials as exceptional nanocarriers for gene therapy, the design of such vectors is still in its infancy. There is therefore a need for more systematic studies, especially in vivo studies, focusing on their safety, biodistribution and efficacy $[34,56]$. 


\subsubsection{Diagnostic applications}

The possibility to use nanomaterials for bioimaging alongside with therapeutic applications is one of the ultimate goals of nanomedicine. In this regard, graphene-based nanomaterials, with intrinsic photoluminescence and NIR responsive properties, have been also exploited for bioimaging of cells and tissues [57].

Altogether, graphene-based materials can be grouped in two major bioimaging categories: optical and non-optical. Among optical bioimaging, graphene based-materials can be used in fluorescence imaging, two-photon fluorescence imaging and Raman imaging, while for non-optical bioimaging, magnetic resonance imaging (MRI), photoacoustic imaging (PAI) and computed tomography (CT) are the most used [58].

(i) Magnetic resonance imaging (MRI)

Among all the bioimaging technics, MRI is widely and the most valuable technique used for imaging and diagnosis in medicine, by allowing a non-invasive visualization of organs and tissues in a quantitative manner [30, 57]. It provides excellent soft tissue contrast without involving ionising radiation, which allows both diagnosis and longitudinal treatment response monitoring [59]. This technique is based on the detection of the relaxation of water protons as they came back to their equilibrium state after being perturbed by a radiofrenquency pulse along with a strong external magnetic field [60]. Although an external contrast agent is not required for image acquisition in MRI, the low sensitivity of the technique makes the use of contrast-enhancing very desirable. Thus, the main task of a contrast agent is to decrease the relaxation time of water protons. In MRI, two main families of contrast agents can be used, $T_{1}$ or longitudinal relaxation (the positive contrast, usually preferred for radiologists) and $T_{2}$ or transverse relaxation (the negative contrast that can disrupt the homogeneity of the magnetic field in their surrounding) [30, 59]. For characterization of contrast agents, relaxivity $r_{1}$ and $r_{2}$ are the most used parameters, which are related with the change of the relaxation rate of water protons per molar concentration of a given contrast agent [30].

Although until today the $T_{1}$ paramagnetic Gadolinium $\left(\mathrm{Gd}^{3+}\right)$ is clinically the most used contrast agent, its high toxicity boost the clinical need to find alternatives [30,60].

Magnetic nanoparticles, especially superparamagnetic iron oxide nanoparticles (SPIONs), have been developed and explored as contrast agents with relaxivities much higher than those obtained by Gd-chelates and with lower toxic effect [30, 54]. In general, SPIONs are composed by nanosize nanoparticles of magnetite or maghemite with predominantly $T_{2}$ relaxation effects, which creates negative dark contrast [60]. However, these nanomaterials 
suffer from the same issues that drawback their safe use in therapeutic techniques like magnetic hyperthermia, i.e., low colloidal stability, tendency to aggregate and precipitate inside blood vessels and low biocompatibility [23]. To overcome these issues, a new generation of coated SPIONs or $\mathrm{Gd}^{3+}$ with different nanomaterials are being investigated.

Graphene-based nanomaterials, such as carboxyphenylated graphene nanoribbons chelated with $\mathrm{Gd}^{3+}$ ions, have shown the ability to enhance MRI relaxivity, $r_{1}$ and $r_{2}, 16$ and 21 times greater than the current clinically available $\mathrm{Gd}^{3+}$-based $T_{1}$ agents [61]. Also, SPIONs combined with GO nanosheets were explored with success as a composite for MRI enhancement contrast with better results compared with single SPIONs [62]. Although carbon materials without the incorporation of magnetic nanoparticles do not show MRI contrast enhancement, recent studies revealed that fluorinated graphene oxide can be used as efficient MRI contrast agent [63].

These studies show that additionally to the ability of using graphene-based materials as protective shells/platforms for SPIONs or paramagnetic transition metal ion chelates for MRI contrast agents, the combination of both materials allows to overcome toxic and/or agglomerative issues of MNPs. Also, the high specific area of carbonaceous shells allows the additional functionalization with active tumour targeting ligands, such as oligosaccharides, oligopeptides, folic acid or antibodies, to increase the cellular uptake of nanoparticles. This approach can allow the selective targeting of cancer cells to simultaneous detect and destroy them with combined therapeutic techniques (e.g. drug delivery, magnetic hyperthermia and/or gene therapy), even when they are disseminated as metastatic cells [54].

\subsubsection{Classification and synthesis techniques}

Magnetic carbon nanostructures are hybrid nanomaterials categorized, in general, in two main classes: (i) carbon encapsulated MNPs and (ii) carbon nanostructures decorated with MNPs [30], as shown in Figure 2.5.

\section{(i) Carbon encapsulated MNPs}

This combined structure is mainly composed by a carbonaceous shell covering a magnetic nanomaterial with spherical or oblong (e.g. nanotubes) geometries that can be designed with core-shell or yolk-shell configurations.

Yolk-shell nanostructures (YSNs), as shown in Figure 2.5 (a), are a special class of core-shell structures composed by a core@void@shell configuration. 
(a)

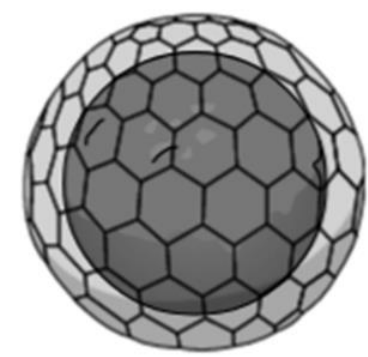

(b)

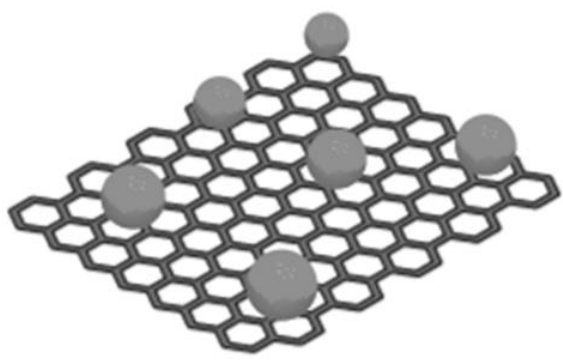

Figure 2.5. Representation of the two main general classes of magnetic carbon nanostructures: (a) carbon encapsulated MNPs (yolk-shell configuration), and (b) carbon nanostructures decorated with MNPs.

YSNs have been received great attention due to its hollow space, which shows great potential to be used as optimized nanocarriers for drug/gene delivery applications, nanoreactors, biosensors, among others [64].

These heterogenic structures can be synthesized by direct routes (also known as in situ encapsulation), such as chemical vapor deposition (CVD), or by indirect routes, mostly using wet chemical techniques [30]. In the second route, a multistage synthesis is required, generally divided by a first step, where the magnetic core is synthesized and followed by a second step, where the magnetic core is encapsulated with the carbonaceous shell.

Overall, the main advantage of the encapsulation technique is the ability to protect the magnetic core from corrosion, as well as to avoid the toxic side-effect of the magnetic core. Additionally, the carbonaceous shell allows the possibility to functionalize the combined nanostructure accordingly with specific needs of the final application.

\section{(ii) Carbon nanostructures decorated with MNPs}

In this magnetic composite nanostructure, MNPs can decorate a broad variety of carbonaceous nanostructures. In contrast with the previous heterogenic structure, MNPs are not protected against the environment nor is the environment protected against their possible toxicity. In this case, the carbon platform acts as a nanocarrier for nanomagnets and other biomolecules [30]. Also, in this case, additional functionalization of the nanoparticles is restricted to the space occupied by these magnetic nanoparticles.

Depending on the type of composite nanostructures and final application, several synthetic routes were developed in the last decade envisioning an efficient shape-control, colloidal stabilization and well-defined magnetic carbon hybrid nanostructure. Among them, the most popular includes filling processes, template-based synthesis, chemical vapor deposition, hydrothermal/solvothermal method, pyrolysis, sol-gel process, detonation induced reaction and self-assembly method [31]. 
Independently on the type of synthesis that is selected to produce these hybrid nanostructures, it is mandatory for biomedical applications that those structures are well characterized, with especial concern regarding their toxicity, biocompatibility and interaction with human cells (i.e. blood cells, different tissues and organs). In fact, despite of all the progress made in the last decade for the development of efficient nanoplatforms for theranostic applications, in general the biocompatibility and haematological effect of these multifunctional structures are not reported or fully investigated [65]. The main reason for this lack of biological studies can be attributed to the difficulty to obtain good models that allow the collection of easier and readable data. Typically, the screening of such materials involves 2D cell/tissue cultures or the sacrifice of small animal (e.g. mice, rats and rabbits) [66]. In this last alternative, besides the ethical concern that rises from the use of animals, these models have shown to be inefficient to predict human response, creating a slow pace for the translation of these nanosystems for clinical applications [67].

As an alternative to these models, microfluidic devices have been explored as useful tools capable to give new insight into chemical, physical and biological response of cells. Indeed, microfluidics have been considered a technology that has the potentiality to significantly change the way modern biology is performed and an optimal platform capable to improve diagnosis and clinical research [68].

\subsection{Microfluidic devices}

Microfluidic devices (Figure 2.6) can be defined as the set of technologies handling and processing small fluid volumes (e.g., $\mu \mathrm{L}, \mathrm{nL}$ and $\mathrm{pL}$ ) through microchannel geometries, with dimension of tens to hundreds of micrometres embedded in a chip [69-72]. Related with these characteristics, since small amounts of reagents and samples are used, microfluidic devices are suitable for analytical purposes with several advantages, such as short times for analysis, reduction of reagent costs, low fabrication cost, miniaturization, sensitivity, selectivity, repeatability, portability and biocompatibility [70, 72]. Furthermore, microfluidic devices can be used as an integrative multiple processes device, called as labon-a-chip (LoC) or micro total analysis system ( $\mu$ TAS). This concept of "miniaturized total chemical analysis system", known in our days as $\mu$ TAS or LoC, was introduced by Andreas Manz and co-workers in 1990 [73]. Since then, the scientific expectations on this technology and analytical possibilities have increased as analytical tool [72], but also as a tool capable to improve the global health $[74,75]$. Some application examples at the laboratory scale are found in clinical diagnosis, such as point-of-care testing [76-78], environment monitoring 
[79-81], food industry [82, 83], microelectronics [84, 85] and in numerous biochemical and biological processes (e.g., analysis of blood samples [86], drug screening [87], cell counting and sorting [88], cell culture studies [89], polymerase chain reaction (PCR) [90], DNA sequencing [91], among others).

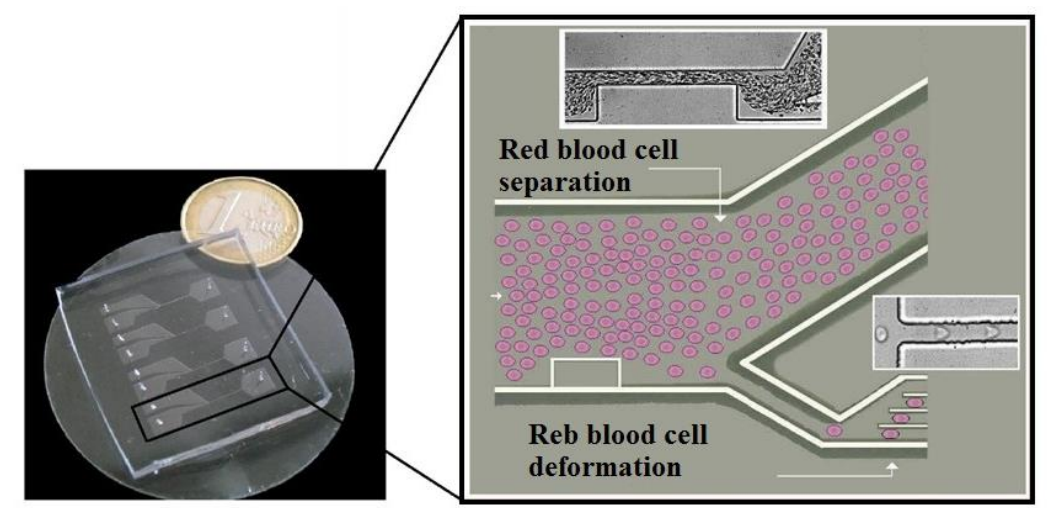

Figure 2.6. Representation of a microfluidic device combining red blood cells separation and deformation for clinical diagnosis applications.

\subsubsection{Microfluidics in biomedical research}

More than a decade ago, Beebe and co-workers [93] declared that, "microfluidics have the potential to significantly change the way modern biology is performed". This optimism around microfluidics was supported by several remarkable advantages that this microscale approach has over traditional assays used in cell biology, offering a better representation of the physiological and pathological conditions of complex biological systems $[68,94]$. In addition to this biomimetic capability, microfluidics allow the possibility to integrate micro-electrochemical systems (MEMS), automatization systems and/or culture cells. In this way, the versatility of microfluidic devices promotes the investigation of a variety of biological systems from single-cell biophysical characterization, to miniaturization of an entire laboratory onto a single chip (i.e., lab-on-a-chip), and more recently, the recapitulation of the organs physiological parameters into a chip (i.e. organ-on-a-chip). All these possibilities make possible a dramatic shift in the way that diagnosis and treatment are performed in medicine and thus, in human health.

\subsubsection{Single-cell biophysical studies}

Biophysical properties of cells (i.e. cell size, mechanical and electrical properties) have important roles in several biological processes, such as metabolic activities, 
regulation of gene expression, differentiation, migration, among others [95]. Changes in these biophysical and/or biochemical properties can help to identify pathological cells and precociously diagnose several diseases. On the contrary of conventional techniques that are time-consuming with tedious operation procedures and very low measurement throughput, microfluidic techniques, with inherently small sample volumes, integration capability and fast response, are very attractive for the study and analysis of cells [95, 96]. However, the great advantage of this technology for cell studies lies on the physiological phenomena that happens at the microscale. Is important to remind that at the microscale level, different forces become dominant over those experienced at the macroscale. Among the most important effects that become dominant at the microfluidic scale are the laminar flow, diffusion, fluidic resistance, surface area to volume ratio and surface tension [96]. Therefore, the design of new microfluidic chips should have those phenomena into account to take advantages of such forces to enhance microscale devices.

At the microscale level, the structure of cells (e.g. cell wall, nucleus and cytoskeleton) and its mechanical properties are the fundamental basis for the understanding of cell's biology. For instance, it is well known that many pathologies can alter the cytoskeleton composition of cells, by reorganizing their network structure and changing their protein density [95]. This results in the change of cell deformability, which plays a crucial role in the mobility of cancer cells [97], and in the deformability of blood cells [98, 99]. Indeed, the decrease in the deformability of red blood cells (RBCs) has been proven to be relevant in the identification of several human diseases, such as malaria [100], diabetes [101], hereditary spherocytosis [102], among others.

For the measurement and characterization of the mechanical properties of cells, several microfluidic techniques based on cell-deformation can be used, namely optical stretcher, DEP-induced deformation, aspiration-induced deformation, electroporation-induced, and fluid-induced deformation based on constriction channels [95].

For the assessment of the deformability of RBCs, microfluidic devices based on fluid-induced deformation are the most used. Unlike other microfluidic techniques that promotes the deformation of cells by using external triggers, such as electrical voltages or laser beam, cells submitted to fluid-induced deformation change their conformation by fluid shear stress $[94,106]$. Since RBCs are highly deformable in small blood vessels under fluid shear stress, most of the microfluidic devices designed for deformability studies are based on transient high shear stress in a sudden-contraction microchannel [103]. However, besides shear stress, extensional flow or the combination of both can be found in the human body at the microvascular level [103]. Indeed, microfluidic channels designed with hyperbolic 
contractions that promote extensional flow, have shown the ability to deform RBCs in a more efficient way than sudden contraction by high shear stress, with the advantage to avoid tumbling and rolling motions of cells [95, 104]. This efficient microfluidic technique with the aid of high-speed imaging or electrical impedance measurements is also recognized to allow multiple parameter outputs along with the deformability of cells, such as transit time, elongation and recovery time [94, 104].

At the microfluidic level, RBCs when subjected to high shear and extensional effects tend to elongate into ellipsoid shapes, aligning their major axis with the flow direction. Then, when the fluid shear forces created by the walls are removed, RBCs tend to return to their normal biconcave disc shape [103]. Due to these merits, fluid-induced deformation channels have been widely used not only to assess the deformability of RBCs [105], but also leukocytes [99, 106] and cancer cells [107]. The quantification of the degree of deformability, known as deformation index (DI) or elongation index, is conventionally calculated using the expression $(X-Y) /(X+Y)$, where $X$ and $Y$ represent the major and minor lengths of the cell in ellipsoid shape, respectively (cf. Figure 2.7 (a)). Nevertheless, if a sudden contraction channel is used with a dimension lower than those of cells, RBCs tend to deform in a parachute shape, and the degree of deformability should be measured in this case, as the ration between $X$ and $Y$ [103], as exemplified in Figure 2.7 (b).

(a)

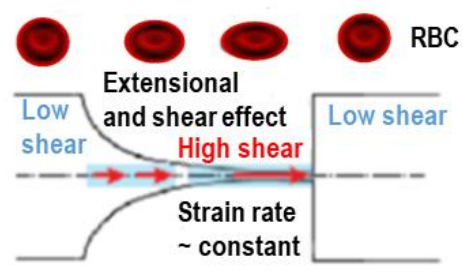

Hyperbolic contraction

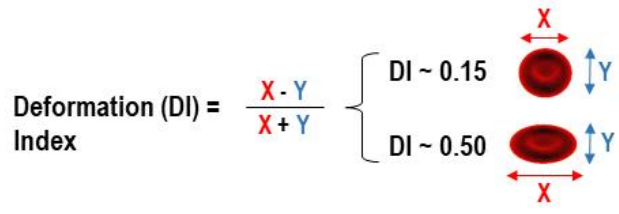

(b)

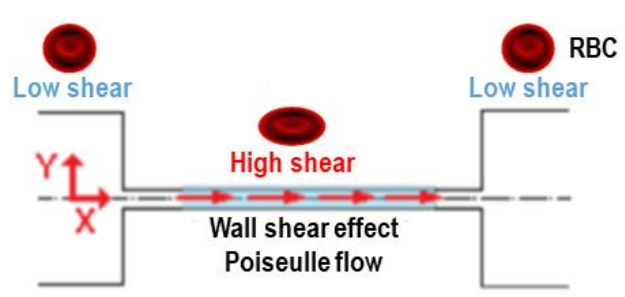

Sudden contraction

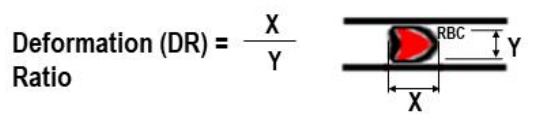

Figure 2.7. Blood flow and RBC deformability equation used for different microfluidic induced-fluid contractions: (a) Hyperbolic contraction and (b) Sudden contraction.

Additional information about the visualization and measurements of RBCs under fluidinduced deformation can be found in Appendix A, where a review published in the journal Micromachines details these topics. Moreover, in Appendix B, a study published in the journal Biomedical microdevices reports the use of a microfluidic device developed for continuous blood cells separation (i.e., RBCs and leucocytes) and deformation assessment using hyperbolic contraction. 


\subsubsection{Lab-on-a-chip}

After the pioneering paper of Manz et al. in 1990 [73], where the new concept of $\mu$ TAS was introduced by using silicon chip analysers to combine sample pre-treatment, separation and detection, many companies found new applicability of these systems for life science purposes [108]. Few years later, this new concept provided a novel approach to integrate separated standard laboratory-based assays into a single microdevice, called as LoC, as referred above [109]. With the additional advantage of miniaturization that enhance its analytical performance, these small size devices present other advantages such as short times for analysis, reduction of reagent, low fabrication cost and portability, which allow them to be used as point-of-care $(\mathrm{PoC})$ diagnostic devices for global health applications [7678]. Due to these advantages, the appropriate development of PoC devices for high-impact diseases, such as malaria, HIV/AIDs, tuberculosis, cancer, diabetes or cardiac diseases, is seen as a technology that will allow a significant reduce on the global health burden [110]. Therefore, PoC tests are seen as able to short diagnosis and prompt accurate treatments, especially in settings where timing is critical (such in emergency cases), where low or nonexistent laboratory facilities are presented and/or when professional and financial resources are low [111].

Although in the past decade many achievements have been done on $\mu$ TAS technologies, with outstanding improvements in the field of microfluidics and integration of monitoring techniques, just few $\mu$ TAS devices have been successfully adopted for clinical applications. The main reason of the low spreading of this technology for clinical purposes is the inability to fabricate low cost disposable automatized microdevices, without the need of benchtop assays. Therefore, microfabrication and miniaturization of microelectromechanical systems have a crucial role on the development and spreading of these technologies in the near future $[68,108,111]$.

An exceptional case of PoC success, is the microfluidic-based device i-STAT Portable Clinical Analyser system from Abbot diagnostics, shown in Figure 2.8. This handling device allows the quantification of biological parameters in blood sample, by using microfluidic disposable cartridges with integrated electrochemical sensors, thus reducing the sampling and result time to around $5 \mathrm{~min}[112]$. 


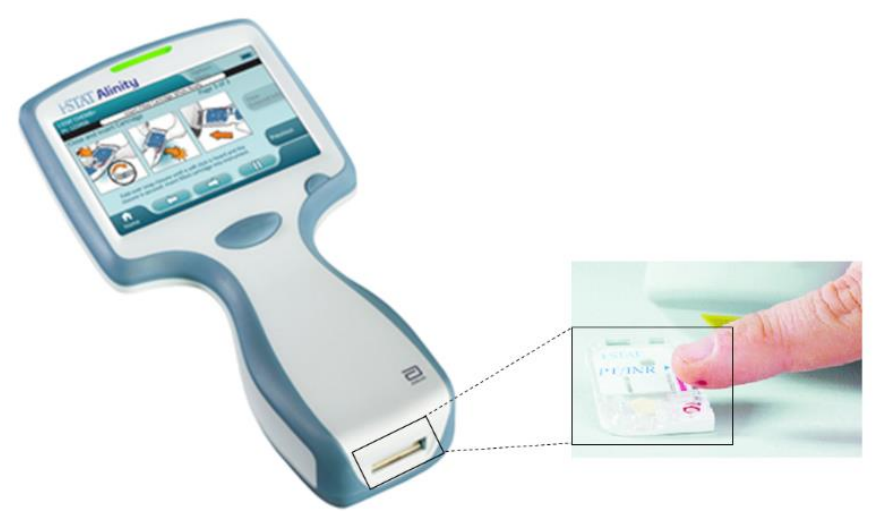

Figure 2.8. Images and handling procedure of i-STAT Portable Clinical Analyser system from Abbot diagnostics. Reprinted from [112]. Copyright $\odot 2010$, with permission from Royal Society of Chemistry.

\subsubsection{Organ-on-a-chip}

Organ-on-a-chip platforms, as represented in Figure 2.9, are a new class of microfluidic devices that have emerged in the recent years, alongside with the development of new 3D printing techniques and tissue engineering, with the purpose to fulfil the limitation of animal studies in predicting clinical outcomes $[113,114]$. Although animal trials are the conventional method applied in early-stage tests to determine biocompatibility and toxicological effect of new drugs, biomaterials and biomedical devices (such as nanoparticles, prosthesis, among others), this methodology is considered exceedingly expensive, time-consuming and most of the times inadequate to represent human physiology $[113,115]$. Therefore, the development of microphysiological systems - i.e, microfluidic devices able to mimic in vivo human biology, have been captivating scientists and receiving a significant financial support, especially from pharma-industries and government agencies [113].

In this context, organ-on-a-chip have emerged as a lab-on-a-chip platform combined with tissue culture techniques, able to produce fluid shear stress, mechanical stress, biochemical concentration gradient and other physical stimuli, with the goal to recapitulate human physiology at lower cost and higher reproducibility [116]. Also, this new methodology overpasses the ethical concern regarding the use of animals for human testing products, which is in line with the 3 Rs' animal principle (reduce, refine and replace animal testing) [113].

In recent years, a variety of human organ models have been developed to mimic different human physiological conditions and organs, such as bone [117], brain [118], eye [119], heart [120], liver [121], lung [122], skin [123], vasculature system [124], among others. But, the ultimate goal for biomedical applications is still the development of multiorgan-on-a-chip 
systems for pharmacology tests and personalized body-on-a-chip for diagnosis and treatment [113]. Alongside with these clinical applications, organ-on-a-chip platforms can play an important role in the boost of nanomaterials developed for nanomedicine [115].

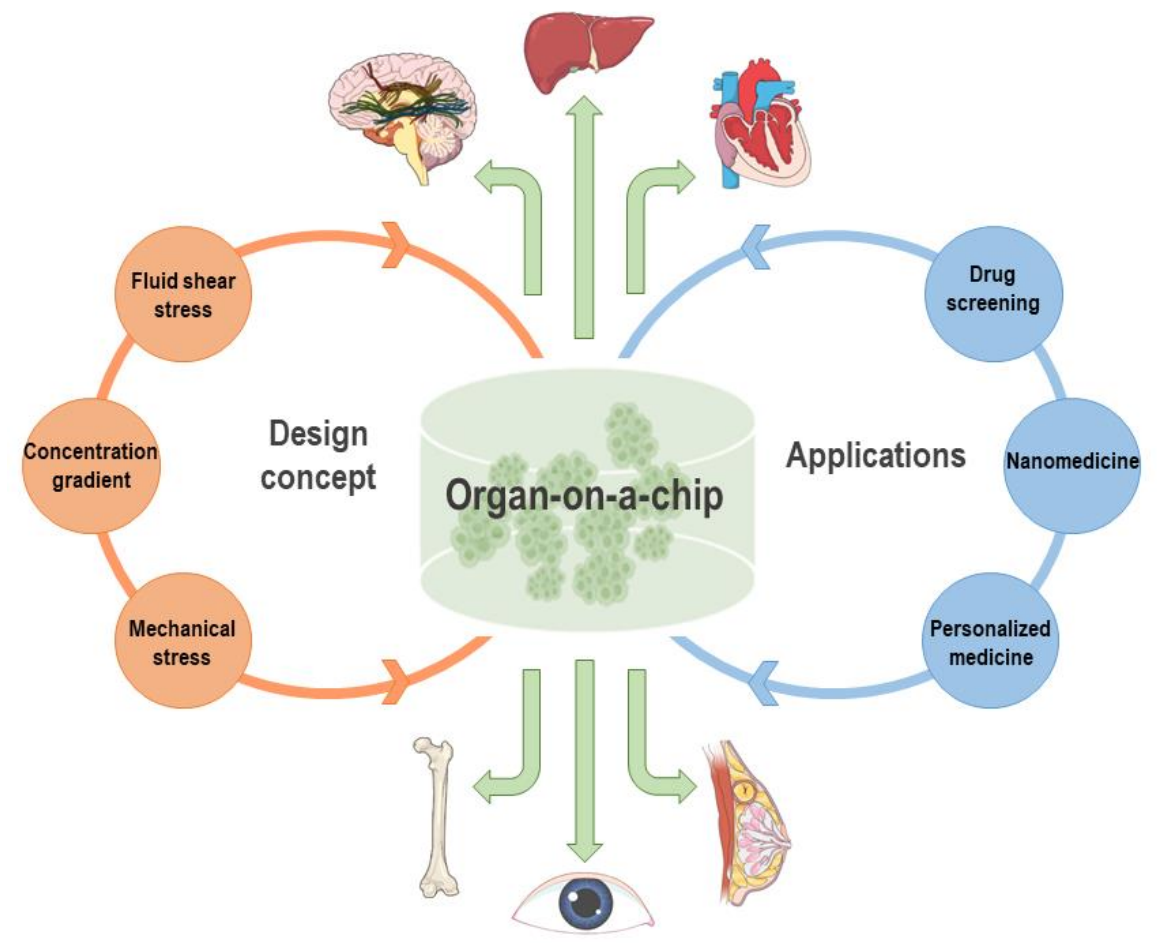

Figure 2.9. Representation of the principal design concept and applications of organ-on-a-chip platforms.

Although the apparent success of nanoparticles developed and tested at laboratory scale is high, the translation of nanomedicine products to clinical applications has been limited and slow [115, 125]. In fact, it is estimated than less than 200 nanomedicine products have been commercialized so far [125]. Moreover, a recent study reported that only $0.7 \%$ of the injected nanoparticles developed for cancer theranostics, are able to reach and accumulate into tumours, which has caused the stagnation of the delivery efficiency of these nanocarriers in medicine over the past decade [126].

Cancer-on-a-chip systems, which are organ-on-a-chip platforms where healthy tissues are replaced by cancer ones, can have a dramatic impact in cancer medicine by mimicking the complex 3D microstructures and extracellular matrix of tumour microenvironment [115]. Due to the close mimic to in vivo cell biology and physiology, these platforms are able to surpass the low accuracy of conventional planar and static cell culture models. Additionally, the potential to couple these platforms with human cells make these systems much more attractive than animal models, especially by its possibility to develop personalized medicine $[67,113]$. Overall, cancer-on-a-chip systems can have a dramatic impact in drug screening, 
therapy efficiency assessment, metastasis studies, personalized medicine and nanomedicine [115].

Surprisingly, although the remarkable potentiality to use these platforms to assess theranostic nanosystems, cancer-on-a-chip platforms have not been intensively implemented. One of the first studies where it was implemented a simple microfluidic device coated with a monolayer of cancer cells goes back to 2005, when Farokhzard and co-workers studied the dynamic interaction of different size nanoparticles with cancer cells [127]. Since then, some prototypes of 3D cancer-on-a-chip platforms have been developed for nanoparticle assessment [128-130]. Although their merits, the effective application of those platforms to assess nanomedicine has been limited, and some challenges are still needed to be addressed: (i) necessity to combine healthy and cancerous organoids interconnected by body or media flow, in order to develop a representative cancer-patient-on-a-chip system; (ii) integration of a functional vasculature network to achieve an accurate testing of the pharmacokinetics and pharmacodynamics of nanoparticles; (iii) incorporation of blood cells and immunocompetent microenvironments to study their interaction with nanoparticles; (iv) design of the proper location and scaling of the different organoids to ensure the human effect; $(v)$ efficient real-time miniaturization of the organs/tissues in the microbioreactors along with extensive period of time [114, 115].

\subsubsection{Microfabrication techniques of microfluidic devices}

Whereas glass and silicon have dominated the early years of $\mu \mathrm{TAS}$, polymers have emerged as a better substrate material for the microfabrication of the microfluidic devices due to its several advantages, such as good optical transparency, biocompatibility, chemical and mechanical properties as well as a friendly system integration (e.g., interconnection with inlets/outlets) [109, 131]. Therefore, these materials enable a high volume of production with good reproducibility, lower cost of fabrication and versatility in the design. These properties are of utmost importance to the creation of disposable microfluidic chips for biomedical and clinical applications. Nevertheless, polymers have some limitations regarding their properties or processing techniques in comparison to glass, such as limited operation-temperature range, higher auto-fluorescence and limited surface modification techniques [132]. Nevertheless, the versatility of polymers and microfabrication techniques can help to address those limitations. Among the most popular polymers to make microfluidic devices are polymethylmethacrylate (PMMA), cyclic olefin copolymer (COC), polystyrene (PS), polycarbonate (PC), polyethyleneterephthalateglycol (PETG) and polydimethylsiloxane 
(PDMS) [67, 69-72]. Depending on the polymer substrate as a matrix in a microfluidic device, different microfabrication techniques can be applied, namely injection moulding [132], hot embossing [133], soft-lithography [86], direct laser plotting [134], laser photoablation or laser micromachining [135], photolithography [136], X-ray lithography [137], among other more recent and low-costly microfabrication techniques, as is the case of print-and-peel techniques, such as xurography [138].

Additional details over the microfabrication techniques and polymers most used on the development of microfluidic devices, can be found in Appendix C. Here, a mini-review published in the $U$. Porto Journal of Engineering details the current fabrication techniques used with polymer materials, as well as the recent low-cost techniques that are gaining interest in the field.

Other important aspect to consider in the fabrication of $\mu$ TAS devices, is the integration of interfaces and interconnections. In general, the following elements should be well integrated for a "plug and play" system: (i) fluid handling unit, (ii) mixing/dilution unit, (iii) sample concentration unit, (iv) filtration/purification unit, (v) sorting unit, (vi) incubation unit, and (vii) detection unit. Among all, detection unit is one of the most important integrated elements, by allowing the possibility to quantify and monitor the target in continuous and real-time [108, 109]. For the quantification purpose, many available sensors ranging from electrochemical detection, optical detection, mass spectrometry to magneticbased detection techniques can be used $[74,108,139]$. The selection of the detection unit is based on the bioanalytical application. The analytes detected include nucleic acids, proteins, small molecules and cells.

\subsubsection{Bioanalytical analysis and applications of microfluidic devices}

\subsubsection{Nucleic acid analysis}

Nucleic acid analysis (DNA or RNA) is one of the leading applications using microfluidic devices. Since nucleic acids are the building blocks of live, this class of analytes can be used in prenatal diagnosis of disorders, clinical disease diagnosis (i.e., infections, genetic diseases and disease staging) and forensic investigations [111]. Additionally to DNA on-chip detection, another significant application is the DNA amplification using PCR on-chip system [139]. 


\subsubsection{Proteins analysis}

Proteins analysis covers a wide range of clinical applications, including immunoassays such as Enzyme-Linked Immunosorbent Assay (ELISA) tests, enzyme assays, separation of amino acids and peptides. For clinical diagnosis and monitoring diseases, protein specimens can be found in a variety of sample matrices such as whole blood, serum/plasma, saliva and urine [111].

\subsubsection{Small molecules analysis}

Small molecules analysis, such as electrolytes from body fluids, is another class of analyte that can be used to monitor health parameters in disease prevention. In general, these analytes are detected based on electrochemical or optical sensing [111].

\subsubsection{Cellular analysis}

Cellular analysis is often required for disease diagnosis and haematological analysis in PoC testing. For heterogeneous cell suspension, such as whole blood, two main categories for sorting can be used: size- and density-based techniques and affinity-based techniques. Other approach involves the lysing of undesired cells and preservation of target cells in the reaction chamber. These methods are, in general, easily implemented on microfluidic devices and are followed by downstream analysis such as fluorescence microscopy or proteomic/genomic analysis [111, 139]. 


\section{References}

[1] R. Gottheil, in Von der Fakultät für Elektrotechnik und Informationstechnik, RheinischWestfälischen Technischen Hochschule Aachen, 2014.

[2] R. Singh, Journal of Magnetism and Magnetic Materials, 2013, 346, $58-73$.

[3] S. Laurent, S. Dutz, U. O. Häfeli and M. Mahmoudi, Advances in Colloid and Interface Science, 2011, 166, 8-23.

[4] N. Pamme, Lab on a Chip, 2006, 6, 24-38.

[5] D. Banerjee, National Institute of Technology, Rourkela, 2011.

[6] A. Berti, C. Giorgi and E. Vuk, Applied Mathematical Modelling, 2015, 39, 820-837.

[7] M. Mahmoudi, S. Sant, B. Wang, S. Laurent and T. Sen, Advanced Drug Delivery Reviews, 2011, 63, 24-46.

[8] A. Ito, M. Shinkai, H. Honda and T. Kobayashi, Journal of Bioscience and Bioengineering, 2005, 100, 1-11.

[9] A. Jordan, R. Scholz, P. Wust, H. Fähling and F. Roland, Journal of Magnetism and Magnetic Materials, 1999, 201, 413-419.

[10] M. Yigit and Z. Medarova, in Micro and Nano Flow Systems for Bioanalysis, eds. M. W. Collins and C. S. Koenig, Springer New York, 2013, pp. 1-14.

[11] A. Hervault and N. T. K. Thanh, Nanoscale, 2014, 6, 11553-11573.

[12] R. T. Gordon, J. R. Hines and D. Gordon, Medical Hypotheses, 5, 83-102.

[13] A. A. M. Elsherbini, M. Saber, M. Aggag, A. El-Shahawy and H. A. A. Shokier, International Journal of Nanomedicine, 2011, 6, 2155-2165.

[14] A. Chichet, J. Skowronek, M. Kubaszewska and M. Kanikowski, Reports of Practical Oncology and Radiotherapy, 2007, 12, 267-275.

[15] R. D. Issels, L. H. Lindner, J. Verweij, P. Wust, P. Reichardt, B. -C. Schem, S. AbdelRahman, S. Daugaard, C. Salat, C.-M. Wendtner, Z. Vujaskovic, R. Wessalowski, K.-W. Jauch, H. R. Dürr, F. Ploner, A. Baur-Melnyk, U. Mansmann, W. Hiddemann, J.-Y. Blay, P. Hohenberger, The lancet oncology, 2010, 11, 561-570.

[16] K. Sugimach, H. Kuwano, H. Ide, T. Toge, M. Saku and Y. Oshiumi, International Journal of Hyperthermia, 1994, 10, 485-493.

[17] A. A. Petryk, A. J. Giustini, R. E. Gottesman, B. S. Trembly and P. J. Hoopes, International Journal of Hyperthermia, 2013, 29, 819-827.

[18] I. Sharifi, H. Shokrollahi and S. Amiri, Journal of Magnetism and Magnetic Materials, 2012, 324, 903-915.

[19] J. Rivas, M. Bañobre-López, Y. Piñeiro-Redondo, B. Rivas and M. A. López-Quintela, Journal of Magnetism and Magnetic Materials, 2012, 324, 3499-3502.

[20] I. A. Brazovich, Medical Physics Monograph, 1988, 16, 82-111.

[21] Y. V. Kolen'ko, M. Bañobre-López, C. Rodríguez-Abreu, E. Carbó-Argibay, A. Sailsman, Y. Piñeiro-Redondo, M. F. Cerqueira, D. Y. Petrovykh, K. Kovnir, O. I. Lebedev and J. Rivas, The Journal of Physical Chemistry C, 2014, 118, 8691-8701.

[22] M. Veverka, P. Veverka, Z. Jirák, O. Kaman, K. Knížek, M. Maryško, E. Pollert and K. Závěta, Journal of Magnetism and Magnetic Materials, 2010, 322, 2386-2389.

[23] N. Alegret, A. Criado and M. Prato, Current Medicinal Chemistry, 2017, 24, 529-536. 
[24] R. Avazzadeh, E. Vasheghani-Farahani, M. Soleimani, S. Amanpour and M. Sadeghi, Progress in Biomaterials, 2017, 6, 75-84.

[25] R. Ghosh, L. Pradhan, Y. P. Devi, S. S. Meena, R. Tewari, A. Kumar, S. Sharma, N. S. Gajbhiye, R. K. Vatsa, B. N. Pandey and R. S. Ningthoujam, Journal of Materials Chemistry, 2011, 21, 13388-13398.

[26] H. Y. Zhu, R. Jiang, L. Xiao and G. M. Zeng, Bioresource Technology, 2010, 101, 50635069.

[27] X. Yu and Y. Zhu, Science and Technology of Advanced Materials, 2016, 17, 229-238.

[28] J. F. Hainfeld, L. Lin, D. N. Slatkin, F. A. Dilmanian, T. M. Vadas and H. M. Smilowitz, Nanomedicine : nanotechnology, biology, and medicine, 2014, 10, 1609-1617.

[29] H. Wei, W. Yang, Q. Xi and X. Chen, Materials Letters, 2012, 82, 224-226.

[30] S. Boncel, A. P. Herman and K. Z. Walczak, Journal of Materials Chemistry, 2012, 22, 31-37.

[31] M. Zhu and G. Diao, Nanoscale, 2011, 3, 2748-2767.

[32] D. Chen, C. A. Dougherty, K. Zhu and H. Hong, Journal of Controlled Release, 2015, 210, 230-245.

[33] S. Li, J. Zheng, D. Chen, Y. Wu, W. Zhang, F. Zheng, J. Cao, H. Ma and Y. Liu, Nanoscale, 2013, 5, 11718-11724.

[34] S. Goenka, V. Sant and S. Sant, Journal of Controlled Release, 2014, 173, 75-88.

[35] E. J. C. Amieva, J. López-Barroso, A. L. Martínez-Hernández and C. Velasco-Santos, in Recent Advances in Graphene Research, ed. P. Nayak, InTech, 2016.

[36] J. Liu, L. Cui and D. Losic, Acta Biomaterialia, 2013, 9, 9243-9257.

[37] Y. Yang, A. M. Asiri, Z. Tang, D. Du and Y. Lin, Materials Today, 2013, 16, 365-373.

[38] V. C. Sanchez, A. Jachak, R. H. Hurt and A. B. Kane, Chemical Research in Toxicology, 2012, 25, 15-34.

[39] G. Gonçalves, M. Vila, M. T. Portolés, M. Vallet-Regi, J. Gracio and P. A. A. P. Marques, Advanced Healthcare Materials, 2013, 2, 1072-1090.

[40] S. Mohapatra, S. R. Rout, R. K. Das, S. Nayak and S. K. Ghosh, Langmuir, 2016, 32, 1611-1620.

[41] A. R. K. Sasikala, R. G. Thomas, A. R. Unnithan, B. Saravanakumar, Y. Y. Jeong, C. H. Park and C. S. Kim, Scientific Report, 2016, 6, 20543.

[42] M. Gisbert-Garzarán, M. Manzano and M. Vallet-Regí, Bioengineering, 2017, 4, 1-27.

[43] Y. Zhang, L. Han, L.-L. Hu, Y.-Q. Chang, R.-H. He, M.-L. Chen, Y. Shu and J.-H. Wang, Journal of Materials Chemistry B, 2016, 4, 5178-5184.

[44] A. Al-Nahain, S. Y. Lee, I. In, K. D. Lee and S. Y. Park, International Journal of Pharmaceutics, 2013, 450, 208-217.

[45] M. Mahdavi, F. Rahmani and S. Nouranian, Journal of Materials Chemistry B, 2016, 4, 7441-7451.

[46] K. C. Barick, S. Singh, D. Bahadur, M. A. Lawande, D. P. Patkar and P. A. Hassan, Journal of Colloid and Interface Science, 2014, 418, 120-125.

[47] S. Y. Qin, J. Feng, L. Rong, H. Z. Jia, S. Chen, X. J. Liu, G. F. Luo, R. X. Zhuo and X. Z. Zhang, Small, 2014, 10, 599-608. 
[48] Y. Xu, M. Mahmood, A. Fejleh, Z. Li, F. Watanabe, S. Trigwell, R. B. Little, V. P. Kunets, E. Dervishi, A. R. Biris, G. J. Salamo and A. S. Biris, International Journal of Nanomedicine, 2010, 5, 167-176.

[49] G. Battogtokh and Y. T. Ko, Journal of Controlled Release, 2016, 234, 10-20.

[50] Z. Huang, X. Zhou, Y. He, X. Ke, Y. Wen, F. Zou and X. Chen, Scientific reports, 2016, 6, 38072 .

[51] M. V. Pilepich, K. G. Jones, B. N. Emami, C. A. Perez, J. N. Fields and R. J. Myerson, International Journal of Radiation Oncology • Biology • Physics, 16, 211-213.

[52] R. A. Vertrees, G. C. Das, V. L. Popov, A. M. Coscio, T. J. Goodwin, R. Logrono, J. B. Zwischenberger and P. J. Boor, Cancer Biology \& Therapy, 2005, 4, 1144-1153.

[53] K. Komatsu, R. C. Miller and E. J. Hall, British Journal of Cancer, 1988, 57, 59-63.

[54] S. Mornet, S. Vasseur, F. Grasset and E. Duguet, Journal of Materials Chemistry, 2004, 14, 2161-2175.

[55] R. Justin, K. Tao, S. Román, D. Chen, Y. Xu, X. Geng, I. M. Ross, R. T. Grant, A. Pearson, G. Zhou, S. MacNeil, K. Sun and B. Chen, Carbon, 2016, 97, 54-70.

[56] M. Vincent, I. de Lázaro and K. Kostarelos, Gene Therapy, 2016, 24, 123.

[57] S. Roy and A. Jaiswal, Reports in Advances of Physical Sciences, 2017, 01, 1750011.

[58] J. Lin, X. Chen and P. Huang, Advanced Drug Delivery Reviews, 2016, 105, 242-254.

[59] J. Gallo, I. S. Alam, I. Lavdas, M. Wylezinska-Arridge, E. O. Aboagye and N. J. Long, Journal of Materials Chemistry B, 2014, 2, 868-876.

[60] J. Gallo, N. J. Long and E. O. Aboagye, Chemical Society Reviews, 2013, 42, 7816-7833.

[61] A. Gizzatov, V. Keshishian, A. Guven, A. M. Dimiev, F. Qu, R. Muthupillai, P. Decuzzi, R. G. Bryant, J. M. Tour and L. J. Wilson, Nanoscale, 2014, 6, 3059-3063.

[62] W. Chen, P. Yi, Y. Zhang, L. Zhang, Z. Deng and Z. Zhang, ACS Applied Materials \& Interfaces, 2011, 3, 4085-4091.

[63] Y. H. Hu, Small, 2014, 10, 1451-1452.

[64] J. Liu, S. Z. Qiao, J. S. Chen, X. W. Lou, X. Xing and G. Q. Lu, Chemical Communications, 2011, 47, 12578-12591.

[65] R. O. Rodrigues, M. Bañobre-López, J. Gallo, P. B. Tavares, A. M. T. Silva, R. Lima and H. T. Gomes, Journal of Nanoparticle Research, 2016, 18, 1-17.

[66] V. Giridharan, Y. Yun, P. Hajdu, L. Conforti, B. Collins, Y. Jang and J. Sankar, Journal of Nanomaterials, 2012, 2012, 14.

[67] N. S. Bhise, J. Ribas, V. Manoharan, Y. S. Zhang, A. Polini, S. Massa, M. R. Dokmeci and A. Khademhosseini, Journal of Controlled Release, 2014, 190, 82-93.

[68] E. K. Sackmann, A. L. Fulton and D. J. Beebe, Nature, 2014, 507, 181.

[69] S. Halldorsson, E. Lucumi, R. Gómez-Sjöberg and R. M. T. Fleming, Biosensors and Bioelectronics, 2015, 63, 218-231.

[70] R. Monošík and L. Angnes, Microchemical Journal, 2015, 119, 159-168.

[71] S. K. Sia and G. M. Whitesides, Electrophoresis, 2003, 24, 3563-3576.

[72] G. M. Whitesides, Nature, 2006, 442, 368-373.

[73] A. Manz, N. Graber and H. M. Widmer, Sensors and Actuators B: Chemical, 1990, 1, 244-248. 
[74] A. van Reenen, A. M. de Jong, J. M. J. den Toonder and M. W. J. Prins, Lab on a Chip, 2014, 14, 1966-1986.

[75] P. Yager, T. Edwards, E. Fu, K. Helton, K. Nelson, M. R. Tam and B. H. Weigl, Nature, 2006, 442, 412-418.

[76] J. Do, S. Lee, J. Han, J. Kai, C.-C. Hong, C. Gao, J. H. Nevin, G. Beaucage and C. H. Ahn, Lab on a Chip, 2008, 8, 2113-2120.

[77] J. Liu, B. Du, P. Zhang, M. Haleyurgirisetty, J. Zhao, V. Ragupathy, S. Lee, D. L. DeVoe and I. K. Hewlett, Biosensors and Bioelectronics, 2014, 61, 177-183.

[78] P. Novo, V. Chu and J. P. Conde, Biosensors and Bioelectronics, 2014, 57, 284-291.

[79] H.-H. Jeong, S.-G. Jeong, A. Park, S.-C. Jang, S. G. Hong and C.-S. Lee, Analytical Biochemistry, 2014, 446, 90-95.

[80] A. Mehta, H. Shekhar, S. H. Hyun, S. Hong and H. J. Cho, Water Science and Technology, 2006, 53, 403-410.

[81] G. Sun, P. Wang, S. Ge, L. Ge, J. Yu and M. Yan, Biosensors and Bioelectronics, 2014, 56, 97-103.

[82] C. F. Fronczek, D. J. You and J.-Y. Yoon, Biosensors and Bioelectronics, 2013, 40, 342349.

[83] Y. Zhang, P. Zuo and B.-C. Ye, Biosensors and Bioelectronics, 2015, 68, 14-19.

[84] R. Catalano, G. Perozziello, G. Simone, P. Candeloro, F. Gentile, M. L. Coluccio, F. Pardeo, M. Burghammer, G. Cuda, C. Riekel and E. Di Fabrizio, Microelectronic Engineering, 2014, 124, 13-16.

[85] C. M. Daikuzono, C. A. R. Dantas, D. Volpati, C. J. L. Constantino, M. H. O. Piazzetta, A. L. Gobbi, D. M. Taylor, O. N. Oliveira Jr and A. Riul Jr, Sensors and Actuators B: Chemical, 2015, 207, Part B, 1129-1135.

[86] R. Lima, S. Wada, S. Tanaka, M. Takeda, T. Ishikawa, K. Tsubota, Y. Imai and T. Yamaguchi, Biomed Microdevices, 2008, 10, 153-167.

[87] F. Nason, E. Morganti, C. Collini, C. Ress, S. Bersini, G. Pennati, F. Boschetti, A. Colombini, G. Lombardi, G. Banfi, L. Lorenzelli and G. Dubini, Microelectronic Engineering, 2011, 88, 1801-1806.

[88] E. D. Pratt, C. Huang, B. G. Hawkins, J. P. Gleghorn and B. J. Kirby, Chemical Engineering Science, 2011, 66, 1508-1522.

[89] J. Shi, L. Liu and Y. Chen, Microelectronic Engineering, 2011, 88, 1693-1697.

[90] X. Pan, L. Jiang, K. Liu, B. Lin and J. Qin, Analytica Chimica Acta, 2010, 674, 110-115.

[91] B. M. Paegel, R. G. Blazej and R. A. Mathies, Current Opinion in Biotechnology, 2003, 14, 42-50.

[92] R. O. Rodrigues, R. Lima, H. T. Gomes and A. M. T. Silva, U.Porto Journal of Engineering, 2015, 1, 67-79.

[93] David J. Beebe, a. Glennys A. Mensing and G. M. Walker, Annual Review of Biomedical Engineering, 2002, 4, 261-286.

[94] A. Webster, J. Greenman and S. J. Haswell, Journal of Chemical Technology \& Biotechnology, 2011, 86, 10-17.

[95] Y. Zheng, J. Nguyen, Y. Wei and Y. Sun, Lab on a Chip, 2013, 13, 2464-2483.

[96] D. J. Beebe, G. A. Mensing and G. M. Walker, Annual Review of Biomedical Engineering, 2002, 4, 261-286. 
[97] J. Chen, J. Li and Y. Sun, Lab on a Chip, 2012, 12, 1753-1767.

[98] A. M. Forsyth, J. Wan, W. D. Ristenpart and H. A. Stone, Microvascular Research, 2010, 80, 37-43.

[99] R. Rodrigues, D. Pinho, V. Faustino and R. Lima, Biomedical microdevices, 2015, 17, 19.

[100] H. W. Hou, A. A. S. Bhagat, A. G. Lin Chong, P. Mao, K. S. Wei Tan, J. Han and C. T. Lim, Lab on a Chip, 2010, 10, 2605-2613.

[101] R. Agrawal, T. Smart, J. Nobre-Cardoso, C. Richards, R. Bhatnagar, A. Tufail, D. Shima, P. H. Jones and C. Pavesio, Scientific reports, 2016, 6, 15873.

[102] J. Picot, P. A. Ndour, S. D. Lefevre, W. E. Nemer, H. Tawfik, J. Galimand, L. D. Costa, J. A. Ribeil, M. d. Montalembert, V. Brousse, B. L. Pioufle, P. Buffet, C. L. V. Kim and O. Français, American Journal of Hematology, 2015, 90, 339-345.

[103] D. Bento, R. Rodrigues, V. Faustino, D. Pinho, C. Fernandes, A. Pereira, V. Garcia, J. Miranda and R. Lima, Micromachines, 2018, 9, 151.

[104] C. Xue, J. Wang, Y. Zhao, D. Chen, W. Yue and J. Chen, Micromachines, 2015, 6, 1457.

[105] J. P. Shelby, J. White, K. Ganesan, P. K. Rathod and D. T. Chiu, Proceedings of the National Academy of Sciences of the United States of America, 2003, 100, 1461814622.

[106] M. J. Rosenbluth, W. A. Lam and D. A. Fletcher, Lab on a Chip, 2008, 8, 1062-1070.

[107] H. W. Hou, Q. S. Li, G. Y. H. Lee, A. P. Kumar, C. N. Ong and C. T. Lim, Biomedical microdevices, 2009, 11, 557-564.

[108] Y. Temiz, R. D. Lovchik, G. V. Kaigala and E. Delamarche, Microelectronic Engineering, 2015, 132, 156-175.

[109] D. R. Reyes, D. lossifidis, P.-A. Auroux and A. Manz, Analytical Chemistry, 2002, 74, 2623-2636.

[110] E. Fu, P. Yager, P. N. Floriano, N. Christodoulides and J. McDevitt, IEEE pulse, 2011, 2, 40-50.

[111] C. D. Chin, S. Y. Chin, T. Laksanasopin and S. K. Sia, in Point-of-Care Diagnostics on a Chip, eds. D. Issadore and R. M. Westervelt, Springer Berlin Heidelberg, Berlin, Heidelberg, 2013, pp. 3-21.

[112] D. Mark, S. Haeberle, G. Roth, F. von Stetten and R. Zengerle, Chemical Society Reviews, 2010, 39, 1153-1182.

[113] A. Guan, P. Hamilton, Y. Wang, M. Gorbet, Z. Li and K. S. Phillips, Nature Biomedical Engineering, 2017, 1, 0045.

[114] S. R. Shin, T. Kilic, Y. S. Zhang, H. Avci, N. Hu, D. Kim, C. Branco, J. Aleman, S. Massa, A. Silvestri, J. Kang, A. Desalvo, M. A. Hussaini, S. K. Chae, A. Polini, N. Bhise, M. A. Hussain, H. Lee, M. R. Dokmeci and A. Khademhosseini, Advanced Science, 2017, 4, 1600522.

[115] Y. S. Zhang, Y.-N. Zhang and W. Zhang, Drug Discovery Today, 2017, 22, 1392-1399.

[116] W. Sun, Y.-Q. Chen, G.-A. Luo, M. Zhang, H.-Y. Zhang, Y.-R. Wang and P. Hu, Chinese Journal of Analytical Chemistry, 2016, 44, 533-541.

[117] J. Kuttenberger, E. Polska and B. M. Schaefer, Clinical Oral Investigations, 2013, 17, 1547-1555. 
[118] O. Kilic, D. Pamies, E. Lavell, P. Schiapparelli, Y. Feng, T. Hartung, A. Bal-Price, H. T. Hogberg, A. Quinones-Hinojosa, H. Guerrero-Cazares and A. Levchenko, Lab on a Chip, 2016, 16, 4152-4162.

[119] K. H. Dodson, F. D. Echevarria, D. Li, R. M. Sappington and J. F. Edd, Biomedical microdevices, 2015, 17, 114-114.

[120] A. Agarwal, J. A. Goss, A. Cho, M. L. McCain and K. K. Parker, Lab on a Chip, 2013, 13, 3599-3608.

[121] C. H. Beckwitt, A. M. Clark, S. Wheeler, D. L. Taylor, D. B. Stolz, L. Griffith and A. Wells, Experimental Cell Research, 2018, 363, 15-25.

[122] D. Huh, Annals of the American Thoracic Society, 2015, 12, S42-S44.

[123] M. Wufuer, G. Lee, W. Hur, B. Jeon, B. J. Kim, T. H. Choi and S. Lee, Scientific reports, 2016, 6, 37471.

[124] D. Tsvirkun, A. Grichine, A. Duperray, C. Misbah and L. Bureau, Scientific reports, 2017, 7, 45036.

[125] H. L. Jang, Y. S. Zhang and A. Khademhosseini, Nanomedicine, 2016, 11, 1495-1497.

[126] S. Wilhelm, A. J. Tavares, Q. Dai, S. Ohta, J. Audet, H. F. Dvorak and W. C. W. Chan, Nature Reviews Materials, 2016, 1, 16014.

[127] O. C. Farokhzad, A. Khademhosseini, S. Jon, A. Hermmann, J. Cheng, C. Chin, A. Kiselyuk, B. Teply, G. Eng and R. Langer, Analytical Chemistry, 2005, 77, 5453-5459.

[128] M. M. G. Grafton, L. Wang, P.-A. Vidi, J. Leary and S. A. Lelievre, Integrative biology, 2011, 3, 451-459.

[129] A. Albanese, A. K. Lam, E. A. Sykes, J. V. Rocheleau and W. C. W. Chan, Nature Communications, 2013, 4, 2718.

[130] I. K. Zervantonakis and C. D. Arvanitis, Small, 2016, 12, 2616-2626.

[131] J. Wu and M. Gu, Journal of Biomedical Optics, 2011, 16, 080901-080901-080912.

[132] U. M. Attia, S. Marson and J. R. Alcock, Microfluidics and Nanofluidics, 2009, 7, 1-28.

[133] H. Becker and U. Heim, Sensors and Actuators A: Physical, 2000, 83, 130-135.

[134] L. Wang, R. Kodzius, X. Yi, S. Li, Y. S. Hui and W. Wen, Sensors and Actuators B: Chemical, 2012, 168, 214-222.

[135] J. Rossier, F. Reymond and P. E. Michel, Electrophoresis, 2002, 23, 858-867.

[136] S. Marchesan, C. D. Easton, K. E. Styan, P. Leech, T. R. Gengenbach, J. S. Forsythe and P. G. Hartley, Colloids and Surfaces B: Biointerfaces, 2013, 108, 313-321.

[137] T. Mappes, S. Achenbach and J. Mohr, Microelectronic Engineering, 2007, 84, $1235-$ 1239.

[138] E. Pinto, V. Faustino, R. Rodrigues, D. Pinho, V. Garcia, J. Miranda and R. Lima, Micromachines, 2014, 6, 121-135.

[139] G. S. Fiorini and D. T. Chiu, Biotechniques, 2005, 38, 429-446. 


\section{CHAPTER III Superparamagnetic nanoparticles for theranostic applications}

3.1 Introduction

3.2 Haemocompatibility of iron oxide nanoparticles synthesized for theranostic applications: a high-sensitivity microfluidic tool

3.3 Thermal infrared image processing to assess heat generated by magnetic nanoparticles for hyperthermia applications 



\section{Superparamagnetic nanoparticles for theranostic applications}

\subsection{Introduction}

The development of superparamagnetic nanoparticles for nanomedicine has attracted much attention in the last decades, especially due to their remarkable physicochemical properties acquired at the nanoscale. In particular, superparamagnetic iron oxide nanoparticles have gained increasing importance in the field due to the possibility to combine diagnosis and therapy of cancer, i.e. as contrast agents for MRI and as nanoheaters in magnetic hyperthermia. Nevertheless, the poor heating efficiency of most reported MNPs, together with the lack of biocompatibility and haemodynamic studies, and imprecise experimental determination of the temperature field during hyperthermia treatment, have hamper the spread of multifunctional nanoparticles as the next generation of theranostic bio-agents in medicine.

In this Chapter, the synthesis and optimization of superparamagnetic nanoparticles for nanomedicine are described. For this purpose, the co-precipitation method was selected due to the high yield of nanoparticles synthesized and the respective low toxicity. By ranging synthesis temperatures, different bare MNPs were obtained. These MNPs were fully characterized regarding their chemical, physical and magnetic properties.

In Section 3.2, a new microfluidic methodology for the haemocompatibility assessment of superparamagnetic nanoparticles synthesized for theranostic applications is detailed. The proposed microfluidic tool aims to add a new dimension into the field of nanomedicine, allowing to be applied as a high-sensitivity technique capable of bringing a better understanding of the biological impact of nanoparticles developed for clinical applications. This study was published in the Journal of Nanoparticle Research [1].

In Section 3.3, thermal infrared image processing was considered to assess the heat generated by the developed magnetic nanoparticles during hyperthermia using a home-made AMF apparatus. The imaging processing methodology described in this section, is proposed to be used as a screening tool to evaluate the potentiality of MNPs synthesized for hyperthermia. The results of this study were published as a book chapter in Advances in Visual Computing: Lecture Notes in Computer Science [2]. 


\subsection{Haemocompatibility of iron oxide nanoparticles synthesized for theranostic applications: a high-sensitivity microfluidic tool}

The poor heating efficiency of most reported MNPs, allied to the lack of comprehensive biocompatibility and haemodynamic studies, hamper the spread of multifunctional nanoparticles as the next generation of therapeutic bio-agents in medicine. Despite the large number of reports on the development of MNPs for theranostic applications [3-10], just a few of them report biocompatibility studies $[6,8,10,11]$ and even less report their haemocompatibility $[6,8]$. In fact, the determination of haemocompatibility is crucial to understand the real in vivo potential of any nanomaterial envisioned for systemic administration, due to its potential toxicological reactions (e.g., embolization, haemolysis and coagulation). In addition, haemocompatibility studies have shown to be more sensitive than animal cell toxicity assays to this purpose [6]. Curiously, there is a lack of reports regarding the complex nanoparticle-RBCs membrane interaction, possibly due to the small size of the nanoparticles, which makes very challenging to explore, even in vitro, the haemodynamic and the complex vascular environment [12]. In this context, this section presents, besides the development and characterization of superparamagnetic nanoparticles for theranostic applications, the development of a new microfluidic tool capable to evaluate, in mimicked environments (close to in vivo microvessels), the biocompatibility and toxicity impact of biomedical-oriented MNPs. By combining this microfluidic tool with a highspeed video microscopy system, multiple parameters such as transit time, recovery time and cell deformability, which cannot be easily assessed via conventional approaches, can be quantified with great detail.

In the past, constriction channel-based microfluidic geometries based on sudden and narrow channels, were adopted to determine the rheological and hydrodynamic effect of these structures over the elasticity and deformation of the RBCs. Hence, it was observed that this constriction geometries had the ability to early characterize diseases (i.e., malaria, diabetes, anaemia, among others) in blood cells, by determining differences in their natural deformations index (DI) [13]. However, these close-fitting geometries, in the form of long constrictions, also promote the clogging of the channel when the blood cells increase their rigidity [13]. To overcome this limitation, microfluidic devices with a hyperbolic channel based on extensional flow approach were adopted with great success by Lee, et al. [14], Yaginuma, et al. [15] and Faustino, et al. [16]. In these pioneering works, the mechanical, rheological and haemodynamic environment created by the hyperbolic-shaped contractions were exhaustively tested, allowing to conclude that hyperbolic converging microchannels are a more accurate technique to determine the Dls of the flowing RBCs, than shear flow- 
based methodologies. Additionally, it was demonstrated that the deformability of blood cells could be detailed and quantitatively described under a controlled homogeneous extensional flow, without the cell's tumbling and rotational motions usually observed in the shear flow based methodologies $[14,15,17]$.

In line with the aforementioned studies, the proposed microfluidic tool described in this section adds a new dimension into the field of nanomedicine, by allowing a new application of this high-sensitivity technique capable to bring a better understanding of the biological impact of nanoparticles developed for clinical applications.

Overall, this section aims to (1) develop MNPs suitable for the combined diagnosis and treatment of cancer, known as theranostics; (2) determine the haemocompatibility of the designed MNPs using a conventional analysis technique (i.e., haemolysis test); and (3) propose a new microfluidic tool capable to bring a new concept to assess with higher accuracy the haemocompatibility and toxicity effect of MNPs developed for clinical applications. Moreover, and to the best of our knowledge, this was the first time that an experimental microfluidic methodology was tested with the purpose of gaining new insights over the complex nanoparticle-RBCs membrane interaction, mimicking micro-rheological conditions as those observed in in vivo microvascular environments.

\subsubsection{Materials and methods}

\subsubsection{Chemicals}

Ammonium hydroxide solution $\left(\mathrm{NH}_{4} \mathrm{OH}, 25 \mathrm{wt} \%\right.$ in $\left.\mathrm{H}_{2} \mathrm{O}\right)$ was purchased from Merck (NJ, USA). Iron (II) chloride tetrahydrate $\left(\mathrm{FeCl}_{2} .4 \mathrm{H}_{2} \mathrm{O}, 99 \%\right)$, Dextran $40\left(\left(\mathrm{C}_{6} \mathrm{H}_{10} \mathrm{O}_{5}\right)_{n}\right)$ and ethanol absolute $\left(\mathrm{C}_{2} \mathrm{H}_{6} \mathrm{O}, 99.8 \%\right)$ were purchased from Sigma-Aldrich (MO, USA). Iron (III) chloride hexahydrate $\left(\mathrm{FeCl}_{3} .6 \mathrm{H}_{2} \mathrm{O}, 97 \%\right)$ was supplied by Panreac (Barcelona, Spain). Alendronic acid ( $\left.\mathrm{Al}, \mathrm{C}_{4} \mathrm{H}_{13} \mathrm{NO}_{7} \mathrm{P}_{2}, 98.0 \%\right)$ and acetone $\left(\mathrm{C}_{3} \mathrm{H}_{6} \mathrm{O}\right.$, 99.5\%) were purchase from $\mathrm{TCl}$ Europe. Physiological salt solution (PSS) with $0.9 \% \mathrm{NaCl}$ was supplied by B. Braun Medical (Germany). All chemicals were of analytical grade and used as received without further purification. All aqueous solutions were prepared in deionized water.

\subsubsection{Synthesis of magnetic nanoparticles}

Briefly, $\mathrm{FeCl}_{3} .6 \mathrm{H}_{2} \mathrm{O}(134 \mathrm{mM}), \mathrm{FeCl}_{2} .4 \mathrm{H}_{2} \mathrm{O}(67 \mathrm{mM})$ and $\mathrm{NH}_{4} \mathrm{OH}(1 \mathrm{M})$ solutions were prepared in deionized water at room temperature. Two samples of magnetite were 
synthesized by alkaline co-precipitation, mixing in $100 \mathrm{~mL}$ of deionized water, the iron precursors in the stoichiometric ratio $1: 2$ of iron (II) and iron (III) salts under vigorous magnetic stirring (C-Mag HS7, IKA). Then, the resultant mixture was heated until the desired temperature $\left(30\right.$ or $55^{\circ} \mathrm{C}$ ). At that point, a basic $\mathrm{NH}_{4} \mathrm{OH}(1 \mathrm{M})$ solution was added drop-wise until $\mathrm{pH} \sim 9$, promoting the co-precipitation of the magnetite nanoparticles. The black solution formed in this process was maintained for $30 \mathrm{~min}$ at the same temperature and stirring conditions, ensuring the complete magnetite crystal formation. After that, the samples of MNPs were subjected to repeated washing steps with deionized water to remove impurities; the last washing step was carried out with absolute ethanol and these particles were dried at $60^{\circ} \mathrm{C}$ overnight. The resulting materials were labelled as MAG30 and MAG55, depending on the heating temperature used ( 30 or $55^{\circ} \mathrm{C}$, respectively).

In this study, hydrophilic MNP ferrofluids were obtained after addition of a solution of alendronic acid (Al, $50 \mathrm{mM}, \mathrm{pH}$ 10) into the colloidal suspensions of MAG30 or MAG55 (1 mg mL $\left.{ }^{-1}\right)$, by using a ratio 4:1 (v/v). The mixtures were sonicated during $15 \mathrm{~min}$ and vigorously stirred for $48 \mathrm{~h}$ at room temperature. The resulting suspensions were centrifuged at $6000 \mathrm{rpm}$ for 5 min (Allegra 64R, Beckman Coulter) and washed with acetone to remove free alendronate from the coated MNPs. The purification procedure was repeated twice. The resulting MNPs@Al (MAG30@Al and MAG55@Al) were dispersed in water, sonicated for $30 \mathrm{sec}$ and stored at room temperature.

\subsubsection{Characterization of magnetic nanoparticles}

(i) Size, crystal structure and chemical composition

Transmission electron microscopy (TEM, LEO/ZEISS 906E) was used to analyse individually the morphology and particle size distribution, MAG30 and MAG55, employing an ImageJ software (1.46r, NIH). For each sample, 120 individual MNPs were randomly measured and the results shown as mean. The crystalline phases of these materials were analysed by X-ray diffraction (XRD) in a PAN'alytical X'Pert PRO equipment (CuKa radiation) equipped with an $X^{\prime}$ Celerator detector and a secondary monochromator, in $\theta / 2 \theta$ geometry. The diffractograms were analysed by Rietveld refinement using PowderCell software. Crystallite sizes were calculated applying Williamson-Hall model for the refinement of results. An inductively coupled plasma - optical emission spectrometer (ICP-OES, ICPE-9000, Shimadzu) was employed to determine the iron content on the MNPs@Al hydrophilic samples. 
(ii) Magnetic and hyperthermia properties

The magnetic properties of the powder samples, MAG30 and MAG55, were investigated with a superconducting quantum interference device (SQUID-VSM) magnetometer from Quantum Design. Hysteresis curves were recorded for magnetic fields between $-20 \mathrm{kOe}$ and $+20 \mathrm{kOe}$ at room temperature $\left(300 \mathrm{~K}\right.$ corresponding to $26.9{ }^{\circ} \mathrm{C}$ ). The temperature dependent magnetization was recorded under a magnetic field of 50 Oe in the temperature range between $-271.2^{\circ} \mathrm{C}(2 \mathrm{~K})$ and $26.9^{\circ} \mathrm{C}(300 \mathrm{~K})$, in both $(\mathrm{ZFC})$ and fieldcooled (FC) samples. The MNPs@Al heating efficiencies were evaluated in a hyperthermia equipment (DM 100 system, nB nanoscale Biomagnetics) by using $1 \mathrm{~mL}$ of MNPs@Al water dispersion $\left(0.4 \mathrm{~g}_{\text {Fe304 }} \mathrm{L}^{-1}\right)$ under an applied oscillating magnetic field of $15.95 \mathrm{kA} \mathrm{m}^{-1}$ at a resonant frequency of $688 \mathrm{kHz}$. The temperature increase was measured with an optical fibre and recorded as a function of time for $10 \mathrm{~min}$.

(iii) Magnetic resonance relaxivity studies

The MRI contrast enhancement efficiency of MNPs@Al was studied by the determination of the $T_{1}$ (longitudinal) and $T_{2}$ (transversal) relaxation times with a fixed field relaxometer (Minispec NMR spectrometer mq60, Bruker Instruments) at a magnetic field strength of $1.41 \mathrm{~T}$. To this purpose, three different Fe concentrated solutions were prepared of each sample (MAG30@Al and MAG55@Al), with Fe concentrations of 0.00, 0.04 and $0.08 \mathrm{mM}$. The $r_{1}$ and $r_{2}$ relaxivities were determined from the slopes of a linear fitting of $1 / T_{1}$ or $1 / T_{2}$ vs. the Fe content of the samples.

\subsubsection{Conventional haemolysis analysis}

Haemolysis induced by nanoparticles treatment was assessed photometrically with a UV-Vis spectrophotometer (T70 spectrometer, PG Instruments Ltd.). Human whole blood from healthy donors were collected into $2.7 \mathrm{~mL}$ tubes (S-Monovette ${ }^{\circledR}$, Sarstedt) containing ethylenediaminetetraacetic acid (EDTA), followed by dilution in a ratio $1: 4(\mathrm{v} / \mathrm{v})$ in PSS. MNPs@Al were further suspended in the diluted whole blood in order to obtain a final concentration of 17.4 and $34.8 \mu \mathrm{g}$ Fe304 $\mathrm{mL}^{-1}$. Positive control was obtained using the whole blood diluted in a ratio $1: 4(\mathrm{v} / \mathrm{v})$ in PSS buffer and the negative control by adding to the diluted whole blood the same volume of deionized water $(1: 1, v / v)$. The solutions with MNPs@Al and controls were incubated at $37^{\circ} \mathrm{C}$ during $1 \mathrm{~h}$ in a shaking water bath. The released haemoglobin was determined after centrifugation at $3000 \mathrm{rpm}$ for $5 \mathrm{~min}$, followed 
by photometric analysis of the supernatant at $540 \mathrm{~nm}$. The haemolysis ratio (HR) was calculated from the optical density (OD) using Equation (3.1) [8]:

$$
H R(\%)=\left(100 \times\left(O D_{\text {test sample }}-O D_{\text {negative control }}\right)\right) /\left(O D_{\text {positive control }}-O D_{\text {negative control }}\right)
$$

The data was calculated from three independent experiments (mean \pm standard deviation).

\subsubsection{Microfluidic studies}

(i) Microfluidic device, experimental setup and parameters

The polydimethylsiloxane (PDMS) microchannels evaluated in this work were fabricated by using a soft-lithographic technique, as previously reported by Lima, et al. [18]. Figure 3.1 shows the main channel of the microfluidic device that was designed to have the following dimensions: $20 \times 400 \times 26389 \mu \mathrm{m}$ (height $\times$ width $\times$ length).

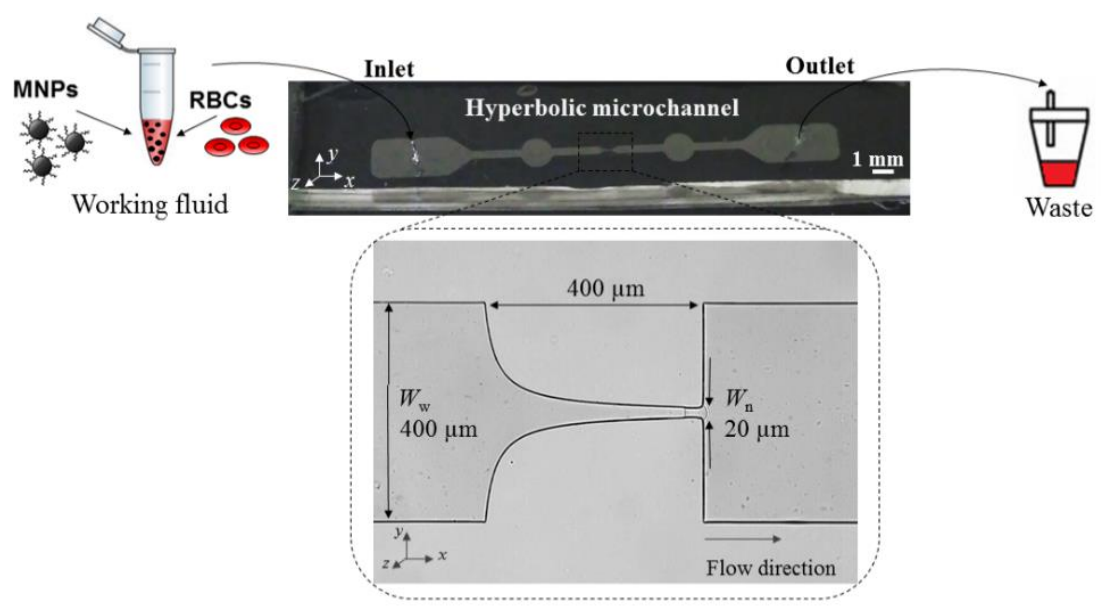

Figure 3.1. Microfluidic device fabricated in PDMS with a hyperbolic-shaped contraction to assess the haemocompatibility of the RBCs in contact with MNPs. Reprinted from [1]. Copyright @ 2016, with permission from Springer Nature.

In the centre of this main channel, a hyperbolic-shaped contraction with a Hencky strain of $\sim 3$ (also called as logarithmic strain, $\varepsilon_{H}=\ln \left(W_{w} / W_{n}\right)$ [17]), was designed to have the following dimensions: $400 \mu \mathrm{m}$ of length and widths of $400 \mu \mathrm{m}$ and $20 \mu \mathrm{m}$, respectively at the wide $\left(W_{w}\right)$ and narrow sizes $\left(W_{n}\right)$. As consequence, the high Hencky strain imposed on this geometry allows an almost linear velocity increase with the axial position, $x$, without suffering entrance or exit effects [17].

The microscopy system used in this work consisted on an inverted microscope (IX71, Olympus) combined with a high-speed camera (Fastcam SA3, Photron, USA). In the inverted microscope, the PDMS microchannel was placed and fixed, and the flow rate of the working 
fluids kept constant at $5 \mu \mathrm{L} \mathrm{min}{ }^{-1}$ by means of a syringe pump (PHD Ultra, Harvard Apparatus, USA) with a $2 \mathrm{~mL}$ syringe (Terumo, Japan). At the same time, the images of the flowing blood cells, at the established flow rate, were captured by the high-speed camera with a frame rate of 2000 frames $\mathrm{s}^{-1}$ and a shutter speed ratio of 1/75 $000 \mathrm{~s}$. These parameters allowed the minimization of some possible image distortions caused by the high flow rate.

Table 3.1 shows the experimental parameters used to perform the haemodynamic study of RBCs as biophysical markers, by assessing their Dls in the described hyperbolic channel.

Table 3.1. Experimental parameters used to perform the haemodynamic study of RBCs, as natural biophysical markers to evaluate the MNPs biocompatibility by assessing their Dis. Reprinted from [1]. Copyright $\odot$ 2016, with permission from Springer Nature

\begin{tabular}{|c|c|}
\hline \multicolumn{2}{|c|}{ Experimental parameters } \\
\hline Maximum width of the microchannel & $400 \mu \mathrm{m}$ \\
\hline Minimum width of the microchannel & $20 \mu \mathrm{m}$ \\
\hline Total length of the contraction region & $400 \mu \mathrm{m}$ \\
\hline Depth of the microchannel & $20 \mu \mathrm{m}$ \\
\hline Inlet flow rate & $5 \mu \mathrm{L} \min ^{-1}$ \\
\hline Mean diameter of the human $\mathrm{RBC}$ at rest & $8 \mu \mathrm{m}$ \\
\hline Shear viscosity of the Dextran 40 & $4.5 \times 10^{-3} \mathrm{~Pa} \mathrm{~s}^{-1}$ \\
\hline Density of the Dextran 40 & $1046 \mathrm{Kg} \mathrm{m}^{-3}$ \\
\hline Haematocrit of the working fluid & $2 \%$ \\
\hline MNPs concentration & 17.4 and $34.8 \mu \mathrm{g}$ Magnetite $\mathrm{mL}^{-1}$ \\
\hline Temperature of the working fluid & $37^{\circ} \mathrm{C}$ \\
\hline Magnification (M) & $20 \times($ Optical zoom $1.6 \times)$ \\
\hline Frame rate & 2000 frames $\mathrm{s}^{-1}$ \\
\hline Exposure time & $500 \mu \mathrm{s}$ \\
\hline
\end{tabular}

(ii) Working fluid protocol

A Dextran 40 solution $(10 \%, w / v)$ containing $2 \%$ of haematocrit (RBCs, v/v) was used as the working fluid to perform the deformability tests in the microfluidic devices. The amount of MNPs@Al was studied at 0.0, 17.4 and $34.8 \mu \mathrm{g}$ Fe304 $\mathrm{mL}^{-1}$. The working fluid prepared without MNPs@Al $\left(0.0 \mu \mathrm{g}\right.$ Fe304 $\left.\mathrm{mL}^{-1}\right)$ was used as positive control.

Briefly, venous human blood samples from healthy donors were collected into $2.7 \mathrm{~mL}$ tubes (S-Monovette ${ }^{\circledR}$, Sarstedt) containing EDTA to avoid coagulation, and centrifuged at $2500 \mathrm{rpm}$ 
for $10 \mathrm{~min}$ at $4{ }^{\circ} \mathrm{C}$. The buffy coat and plasma were removed and the packed RBCs resuspended and washed twice in PSS with $0.9 \% \mathrm{NaCl}$, in a ratio $1: 1(\mathrm{v} / \mathrm{v})$. The final working fluid was prepared immediately before the experiments by mixing together the collected RBCs with the desired amount of MNPs@Al in the Dextran 40 solution (10\%,w/v). Dextran 40 solution was used as carrier fluid instead of the blood plasma to avoid the clotting and jamming of the blood cells in the in vitro microfluidic studies, which are generally promoted by some components, i.e. immunoglobulins, clotting factor, albumin and fibrinogen proteins. The working fluid samples were incubated at $37{ }^{\circ} \mathrm{C}$ during 15,30 and $60 \mathrm{~min}$ in a shaking water bath, allowing the evaluation of the time-contact effect between the MNPs and the deformability of RBCs.

(iii) Image analysis

The experimental images recorded in each test were transferred to a computer, processed and analysed by an image handling software, ImageJ (1.46r, NIH, USA). Using this software, DI of the blood cells was calculated using Equation (3.2) [15]:

$$
D I=L_{\text {major }}-L_{\text {minor }} / L_{\text {major }}+L_{\text {minor }}
$$

For each deformability test performed with three amounts of MNP@Al (0.0, 17.4 and

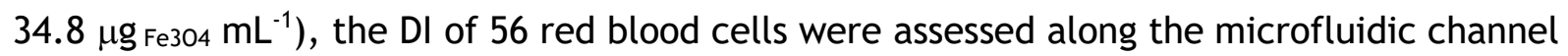
with a hyperbolic-shaped contraction followed by a sudden expansion. The results were displayed as mean \pm standard deviation.

\subsubsection{Ethical approval}

Ethical approval for human blood handling and analysis was given by the medical ethics committee of the Unidade Local de Saúde do Nordeste, E.P.E. (Bragança, Portugal).

\subsubsection{Results and discussion}

\subsubsection{Characterization of the developed MNPs}

(i) Size, crystal structure and chemical composition

The results obtained by TEM analysis of MAG30 and MAG55 are shown in Figure 3.2 , revealing that the magnetic core of the synthesized nanoparticles is nearly spherical for both samples. The increase of the synthesis temperature lead to a growth in the average diameter of the magnetic core, from 11.1 (MAG30) to $17.7 \mathrm{~nm}$ (MAG55), which is in good 52 
agreement with the crystallite sizes obtained by XRD analysis (Table 3.2). These sizes are expected for particles within the single-domain superparamagnetic region.
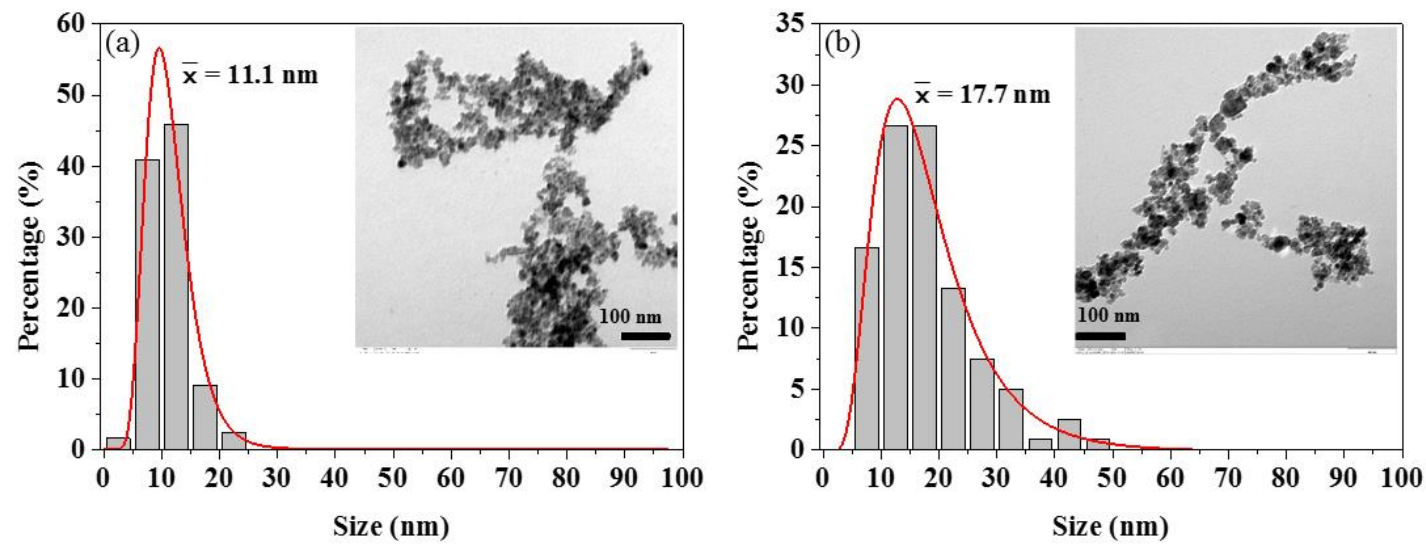

Figure 3.2. Histogram and mean diameter $(\bar{x})$ of the nanoparticles following the Log-normal distribution of (a) MAG30 and (b) MAG55. Inset shows the corresponding TEM images. Reprinted from [1]. Copyright @ 2016, with permission from Springer Nature.

The determination of the nanoparticle size distribution reveals that the average of nanoparticle diameter and size polydispersion grows as synthesis temperature increases. Nevertheless, for the higher synthesis temperature used in this study $\left(55^{\circ} \mathrm{C}\right)$, none of the measured MNPs were found to be larger than $50 \mathrm{~nm}$.

Table 3.2. Physicochemical properties of the synthesized nanoparticles: diameter determined by TEM $\left(\mathrm{d}_{\text {TEM }}\right)$, crystallite size calculated from XRD $\left(\mathrm{d}_{\mathrm{hkl}}\right)$, lattice constant and crystalline core phase obtained by XRD analysis. Reprinted from [1]. Copyright $\odot$ 2016, with permission from Springer Nature

\begin{tabular}{lccccc}
\hline Sample & $\begin{array}{c}\text { Synthesis } \\
\text { method }\end{array}$ & $\begin{array}{c}\mathrm{d}_{\mathrm{TEM}} \\
(\mathrm{nm})^{\mathrm{a}}\end{array}$ & $\begin{array}{c}\mathrm{d}_{\mathrm{hkl}} \\
(\mathrm{nm})^{\mathrm{b}}\end{array}$ & $\begin{array}{c}\text { Lattice } \\
\text { constant, a } \\
(\AA)\end{array}$ & $\begin{array}{c}\text { Crystalline core phases, \% } \\
(\mathrm{v} / \mathrm{v})\end{array}$ \\
\hline MAG30 & Co-precipitation, $30{ }^{\circ} \mathrm{C}$ & 11.1 & 12.8 & 8.3652 & $100 \%$ Magnetite $\left(\mathrm{Fe}_{3} \mathrm{O}_{4}\right)$ \\
MAG55 & Co-precipitation, $55{ }^{\circ} \mathrm{C}$ & 17.7 & 18.5 & 8.3653 & $100 \%$ Magnetite $\left(\mathrm{Fe}_{3} \mathrm{O}_{4}\right)$ \\
\hline
\end{tabular}

a Estimated by using ImageJ software as mean ( $\mathrm{n}=120)$; ${ }^{\mathrm{b}}$ Calculated by using the Williamson-Hall equation [19].

All the reflection peaks of the XRD patterns of MAG30 and MAG55 were indexed by considering magnetite phase. No reflection peaks were observed as impurities or secondary phases. The cubic cell lattice parameter resulted to be $a \sim 8.37 \AA$, in good agreement with the values reported for nanosized magnetite [20]. Also, Table 3.2 shows the crystallite size, which was calculated from the 5 most intense peaks of the XRD patterns through the Williamson-Hall formula [19]. For comparison purposes, the particle diameter observed by TEM is also shown, that resulted to be very similar to that obtained from the XRD data. Thus, co-precipitation method, under mild experimental conditions, revealed to be a suitable synthesis method for biomedical applications. 
(ii) Magnetic and hyperthermia properties

The main magnetic parameters of MAG30 and MAG55, namely saturation magnetization, coercivity and remanence, were calculated from the analysis of the magnetization curves as a function of the applied magnetic field obtained for powdered samples (cf. Table 3.3 and Figure 3.3). The specific absorption rate (SAR, $\mathrm{W} \mathrm{g}^{-1} \mathrm{Fe} \mathrm{O}$ ) values were calculated according to Equation (2.5). In addition, the intrinsic loss power (ILP), independent of the magnetic field amplitude and frequency used, and allowing a direct comparison between different reported values, was calculated using Equation (3.3) [21]:

$$
I L P=\frac{S A R}{H^{2} f}
$$

where $H$ is the field amplitude and $f$ the frequency used in the hyperthermia experimental setup.

Table 3.3. Magnetic properties of the $\mathrm{Fe}_{3} \mathrm{O}_{4}$ nanoparticles synthesized by co-precipitation at different synthesis temperatures $\left(30\right.$ and $\left.55^{\circ} \mathrm{C}\right)$ : saturation magnetization (Ms), coercivity (Hc), saturation remanence (Mr), SAR and ILP. Reprinted from [1]. Copyright @ 2016, with permission from Springer Nature

\begin{tabular}{cccccc}
\hline Sample & $\begin{array}{c}\mathrm{M}_{\mathrm{s}} \\
\left(\mathrm{emu} \mathrm{g}^{-1} \mathrm{Fe304}\right)\end{array}$ & $\begin{array}{c}\mathrm{H}_{\mathrm{c}} \\
(\mathrm{Oe})\end{array}$ & $\begin{array}{c}\mathrm{M}_{\mathrm{r}} \\
\left(\mathrm{emu} \mathrm{g}^{-1} \mathrm{Fe304}\right)\end{array}$ & $\begin{array}{c}\mathrm{SAR}^{\mathrm{a}} \\
\left(\mathrm{W} \mathrm{g}^{-1} \mathrm{Fe} 304\right)\end{array}$ & $\begin{array}{c}\mathrm{ILP}^{\mathrm{b}} \\
\left(\mathrm{nHm}^{2} \mathrm{~kg}^{-1}\right)\end{array}$ \\
\hline MAG30 & $56.19 \pm 0.02$ & 33.65 & 3.77 & 100.18 & 0.57 \\
MAG55 & $77.68 \pm 0.03$ & 18.33 & 1.94 & 565.68 & 3.23
\end{tabular}

a Specific absorption rate (SAR) calculated for $H=15.95 \mathrm{kA} \mathrm{m}^{-1}, f=688 \mathrm{kHz}, \mathrm{C}=0.40 \mathrm{~g} \mathrm{Fe}^{-1} \mathrm{~L}^{-1}$; ${ }^{\mathrm{b}}$ Intrinsic loss power (ILP) independent of the magnetic field parameters, allowing direct comparison between experiments under different experimental conditions.

Both MAG30 and MAG55 hysteresis loops show a superparamagnetic-like behaviour. Whereas MAG30 (11.1 nm of particle size) showed a saturation magnetization, $\mathrm{M}_{\mathrm{s}}$, of $56 \mathrm{emu} \mathrm{g}^{-1}$, the increase of the synthesis temperature in MAG55 led to an increase of the particle size $(17.7 \mathrm{~nm})$ that subsequently resulted in an increase of $M_{s}$, which is near to the bulk value of magnetite, i.e. 90 emu g${ }^{-1}$ [22]. Nevertheless, MAG55 exhibits lower remanence $\left(M_{r}\right)$ and coercive forces $\left(\mathrm{H}_{c}\right)$ at room temperature compared to MAG30, despite the higher particle size of the individual particles (cf. Table 3.2). This unlike result had been previously reported [21] and explained to be caused by the effect of the dipolar magnetic interactions caused by the presence of clustering in small nanoparticles.

On the other hand, the zero-field-cooling - field-cooling (ZFC-FC) magnetization curves shown in Figure 3.3 indicate a shift of the blocking temperature $\left(T_{B}\right)$, defined as the temperature above which the MNPs show superparamagnetic behaviour [23], from - 98.2 to 
$26.9^{\circ} \mathrm{C}$ (or higher) for MAG30 and MAG55, respectively. In both cases, the flatness of the FC curve below $T_{B}$ is indicative of significantly strong dipolar magnetic particle interactions. Hence, the confirmation of a superparamagnetic state for both MAG30 and MAG55 at room temperature make these samples suitable for MRI and hyperthermia applications.
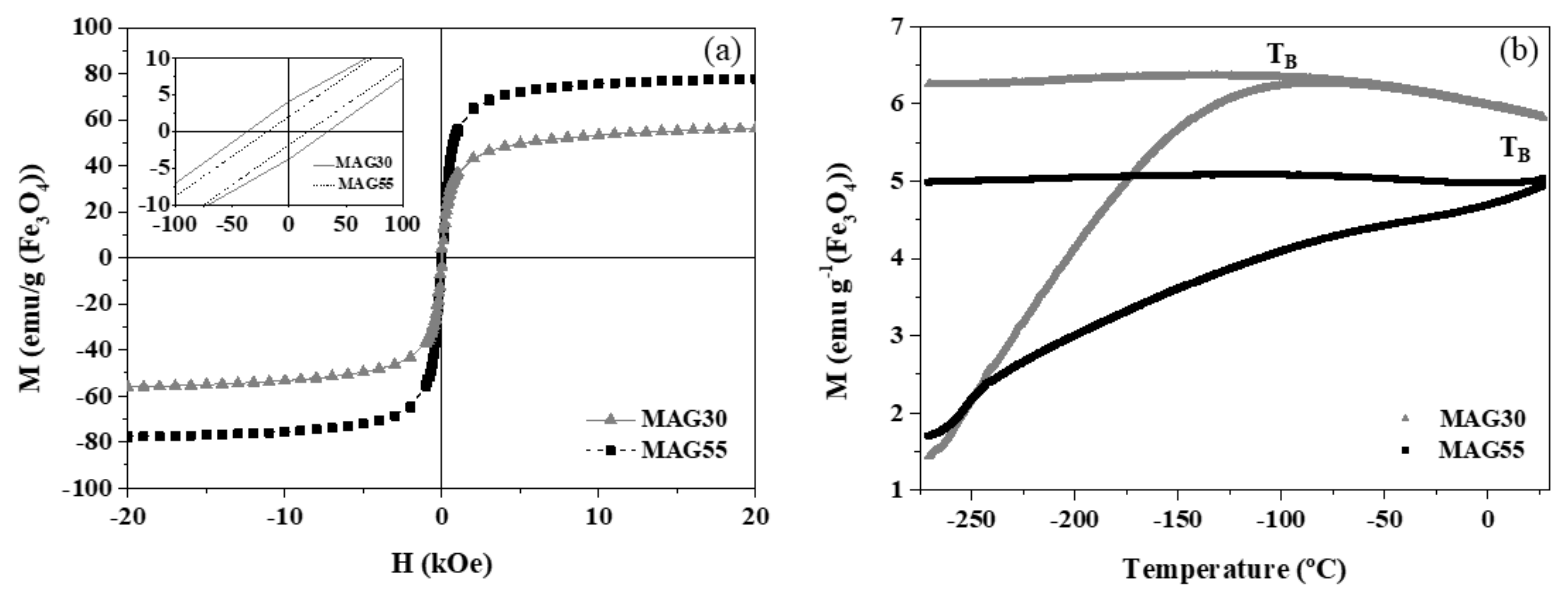

Figure 3.3. Magnetic characterization of MAG30 and MAG55. (a) Hysteresis loops at room temperature up to $\pm 20 \mathrm{kOe}$; the inset is a zoom in the low-field region; (b) ZFC-FC magnetization curves measured at 50 Oe. Reprinted from [1]. Copyright $\odot$ 2016, with permission from Springer Nature.

The hyperthermia performance of MAG30@Al and MAG55@Al nanoparticles was determined in water solution at a concentration of $0.40 \mathrm{~g}$ Fe304 $\mathrm{L}^{-1}$.

Both nanoparticle dispersions were evaluated in terms of SAR and ILP, which are shown in Table 3.3. Figure 3.4 shows the temperature profiles as a function of time for both samples under the same hyperthermia conditions $(H=15.95 \mathrm{kA} / \mathrm{m} ; f=688 \mathrm{kHz})$.

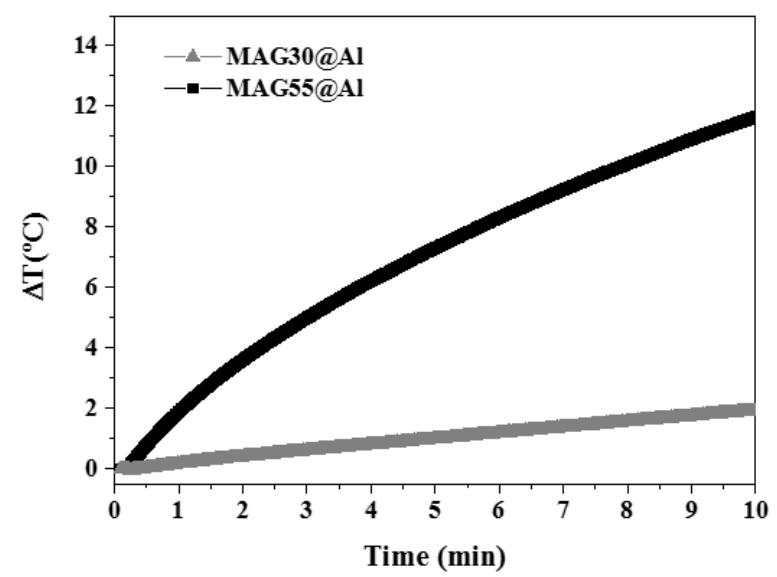

Figure 3.4. Temperature versus time curves for MAG30@Al and MAG55@Al water colloidal dispersions at $0.40 \mathrm{~g} \mathrm{Fe}^{\circ} \mathrm{L}^{-1}$, under an oscillating magnetic field of $15.95 \mathrm{kA} \mathrm{m}^{-1}$ and $688 \mathrm{kHz}$. Reprinted from [1]. Copyright $\odot$ 2016, with permission from Springer Nature.

As expected, SAR increases with the increasing size of the magnetic core of the iron oxide nanoparticles. Indeed, many works compiled by Deatsch and co-workers in 2014 [24] 
indicated that the optimal diameter for magnetite nanoparticles to be applied in magnetic hyperthermia fall somewhere between 12 and $20 \mathrm{~nm}$. However, the comparison between different studies is very difficult since SAR results strongly depend on the magnitude and frequency of the applied magnetic field. Thus, to overcome this issue, ILP was proposed as a measure of heating efficiency that normalizes SAR with respect to field strength, $H$, and frequency, $f$. ILP results revealed a remarkable heating efficiency for the MAG55 nanoparticles $\left(3.23 \mathrm{nHm}^{2} \mathrm{~kg}^{-1}\right)$ when compared with ILP values found for coated magnetite (e.g., mPEGs, chitosan, 2,3-dimercaptosuccinic acid, (3-amino-propyl)triethoxysilane, dextran, among others) $[25,26]$, or reported for commercial ferrofluids, which are in the range between 0.20 and $3.10 \mathrm{nHm}^{2} \mathrm{~kg}^{-1}$ [27]. In fact, it should be noted that the hyperthermia tests performed in this work were made with an extremely low concentration of colloidal

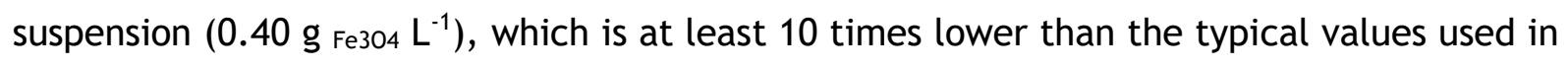
other published magnetic hyperthermia tests [7, 27]. Even at this low concentration, the sample MAG55@Al showed a temperature increment of $12^{\circ} \mathrm{C}$ after just 10 min of exposure to the applied magnetic field. The good ILP result obtained for MAG55@Al is likely due to the combination of narrow size distribution of the synthesized MNPs, good colloidal stability and strong magnetic response caused by the Néel and Brownian power loss mechanisms, dominant and comparable in superparamagnetic nanoparticles.

(iii) Magnetic resonance relaxivity studies

The contrast enhancement efficiency of MNPs@Al was evaluated in water through relaxivity measurements by plotting the relaxation rates $\left(R_{1}=1 / T_{1}\right.$ and $\left.R_{2}=1 / T_{2}\right)$ of water protons in the presence of the nanoparticles, against the Fe content. Upon linear fitting, the relaxivity values, $r_{2}$ and $r_{1}$ were obtained from the respective slope and listed in Table 3.4, as well as the corresponding $R^{2}$ of the linear fit, which shows good fitting results.

Table 3.4. Longitudinal $\left(r_{1}\right)$ and transverse $\left(r_{2}\right)$ relaxivity of MAG30@Al and MAG50@Al. The corresponding coefficient of the linear fit $\left(R^{2}\right)$ and relaxivity ratio $\left(r_{2} / r_{1}\right)$ are also shown. Reprinted from [1]. Copyright (c) 2016, with permission from Springer Nature

\begin{tabular}{cccccc}
\hline Sample & $\begin{array}{c}r_{2}{ }^{\mathrm{a}} \\
\left(\mathrm{mM}^{-1} \mathrm{~s}^{-1}\right)\end{array}$ & $\begin{array}{c}R^{2} \\
\left(\mathrm{r}_{2}\right)\end{array}$ & $\begin{array}{c}r_{1}{ }^{\mathrm{a}} \\
\left(\mathrm{mM}^{-1} \mathrm{~s}^{-1}\right)\end{array}$ & $\begin{array}{c}R^{2} \\
\left(r_{1}\right)\end{array}$ & $r_{2} / r_{1}$ \\
\hline MAG30@Al & 226.08 & 0.9986 & 4.02 & 0.9990 & 56.24 \\
MAG55@Al & 248.17 & 0.9992 & 4.42 & 0.9999 & 56.15 \\
\hline
\end{tabular}

a Relaxivity values measured at $\mathrm{B}=1.41 \mathrm{~T}$ and $37^{\circ} \mathrm{C}$.

Both MAG30@Al and MAG55@Al ferrofluidic samples showed a similar $r_{1}$ of $\sim 4 \mathrm{mM}^{-1} \mathrm{~s}^{-1}$, which is higher than other superparamagnetic iron oxide nanoparticles [7]. More importantly, 
enhanced $r_{2}$ values of $248.17 \mathrm{mM}^{-1} \mathrm{~s}^{-1}$ for MAG55@Al and $226.08 \mathrm{mM}^{-1} \mathrm{~s}^{-1}$ for sample MAG30@Al were achieved, which are in good agreement with other results with surface modified $\mathrm{Fe}_{3} \mathrm{O}_{4}$ nanoparticles [28], and far superior than currently available intravenous iron oxide nanoparticle contrast agents [29]. Moreover, and as expected, these results indicate that the relaxivity values $r_{1}$ and $r_{2}$ increase with the MNPs growth, very likely due to the increase in saturation magnetization.

In MRI, two main families of contrast agents can be used, $T_{1}$ or agents that affect mainly the longitudinal relaxation (positive contrast, brightening of the image) and $T_{2}$ or agents that have an effect predominantly on the transverse relaxation (negative contrast, darkening of the image) [30]. The $r_{2} / r_{1}$ ratio is considered a suitable indicator to assess the efficiency of MNPs as a $T_{1}$ or a $T_{2}$ contrast agent. The $r_{2} / r_{1}$ ratio of MAG30 and MAG55 resulted to be higher than that of some commercial iron oxide nanoparticles contrast agents [29, 31], indicating that both samples are promising candidates for high-efficiency $T_{2}$-weighted MR imaging.

\subsubsection{Conventional haemolysis analysis}

Haemolysis analysis is one of the most common studies performed to evaluate the haemocompatibility of nanomaterials for biomedical applications [8, 32], especially when they are intended to be applied directly into the blood stream. In fact, previous in vitro studies demonstrated that several nanoparticles induced haemolysis, the extent of the effect being directly related to particle size, surface properties and experimental conditions [32].

In the present study, two different contents of MAG55@Al (the sample that shows better performance in both MRI and hyperthermia) were suspended in diluted whole blood and tested, namely 17.4 and $34.8 \mu \mathrm{g}$ Fe304 $\mathrm{mL}^{-1}$. The selection of these concentrations was based on the recommended dosage $\left(15 \mu \mathrm{mol}_{(\mathrm{Fe})} \cdot \mathrm{kg}^{-1}\right.$ of individual $)$ for the commercial superparamagnetic iron oxide-based MRI enhancer (ENDOREM ${ }^{\circledR}$, Guerbet S.A.) in individuals with a weight of 75 and $150 \mathrm{~kg}$, respectively. This choice was made since there are no guidelines available concerning the optimal concentration of iron oxide nanoparticles to be applied systemically for magnetic hyperthermia treatment. Thus, the absorbance results of the supernatant fluids, treated with different concentrations, were compared and normalized with the result from lysed RBCs in the presence of deionized water (taken as $100 \%$ lysed, negative control) and the blood in PSS buffer without MNPs (taken as 0\% lysed, positive control), using for this purpose Equation (3.1). 
According to the standard ISO 10993-4 for the evaluation of biological medical devices suitable for blood contact, the material should have haemolysis rates lower than $5 \%$ [8]. In this work, the haemolysis rates were found to be lower than $2 \%$ for all tested concentrations. More precisely, it was found to be $1.08 \pm 0.73 \%$ for the lowest content of nanoparticles $\left(17.4 \mu \mathrm{g}\right.$ Fe304 $\left.\mathrm{mL}^{-1}\right)$ and $1.63 \pm 0.28 \%$ for the highest $\left(34.8 \mu \mathrm{g} \mathrm{Fe} 304 \mathrm{~mL}^{-1}\right)$. Therefore, and according to the guide for the evaluation of biological materials, the synthesized MNPs developed in this work proved to fulfil the requirements of conventional haemolysis test and suitable to be applied as medical materials for blood contact, even at the highest tested concentration.

\subsubsection{Microfluidic studies}

In this study, and for the first time, a high-sensitivity microfluidic tool capable to assess the deformability of blood cells was used to evaluate the impact of nanoparticles on the human RBCs. This methodology comprises a microfluidic device having a hyperbolicshaped contraction microchannel and a high-speed video microscopy system. This combined system enables the recording, in real time, of the biomechanical microfluidic phenomena, which can be then further analysed to obtain valuable toxicological data.

Figure 3.5 shows a schematic view of the experimental setup, the microfluidic device with the hyperbolic channel and the fluid-induced deformation profiles occurring at the hyperbolic channel, making this technique ideal for DI assessment.

Figure 3.5 (a) represents the high-speed video microscopy system used to perform the microfluidic studies. Moreover, Figure 3.5 (b) and (c) show the fluid-induced deformation profiles that attests that the hyperbolic channel approach is ideal to assess RBC's DI. As previously referred, this extensional flow technique has already shown to be more efficient in deforming and assessing RBC's DI than other techniques based in shear flow [14, 15, 33].

Taking advantage of these previous studies, this extensional flow technique was used for the first time as a microfluidic concept capable of gaining new insights over the complex nanoparticle-RBCs interaction designed for theranostic applications. The main reason for this ability is the promotion of the homogeneous extensional flow along the centreline of the hyperbolic channel with a high Hencky strain. In this type of microfluidic devices, the velocity increases almost linearly and the strain rate is maintained approximately constant, even with the increment of the shear rate [17]. As a result, the technique allows the deformation of the blood cells along the microchannel, causing the RBC stretching by the larger fluid shear stress, without tumbling and rotational motions of the cells, providing a 
more accurate way to determine the DI of the blood cells, mimicking in vitro microvascular environments.

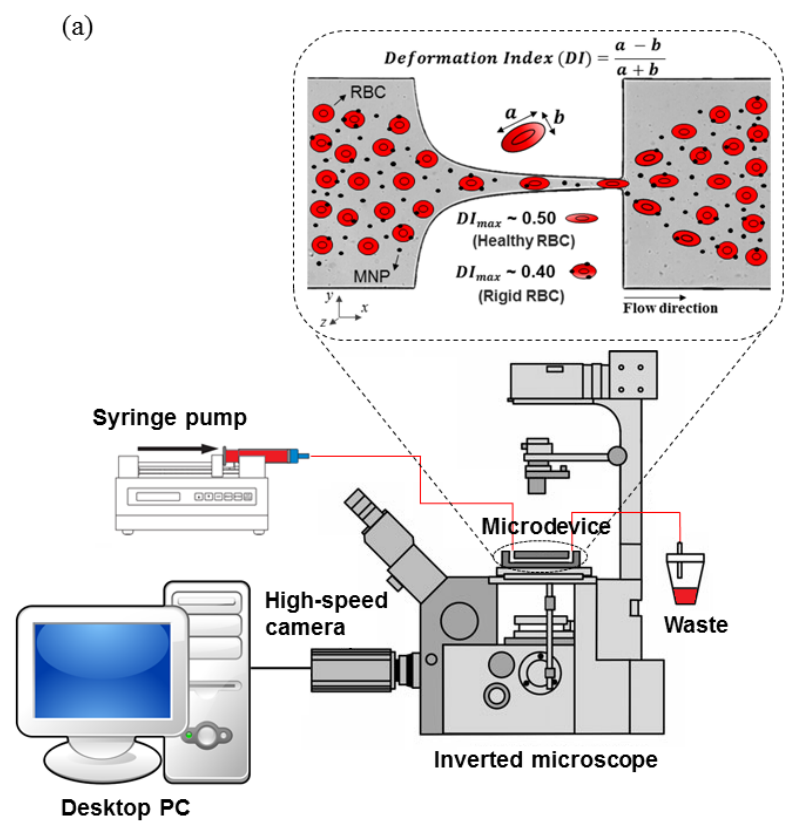

(b)
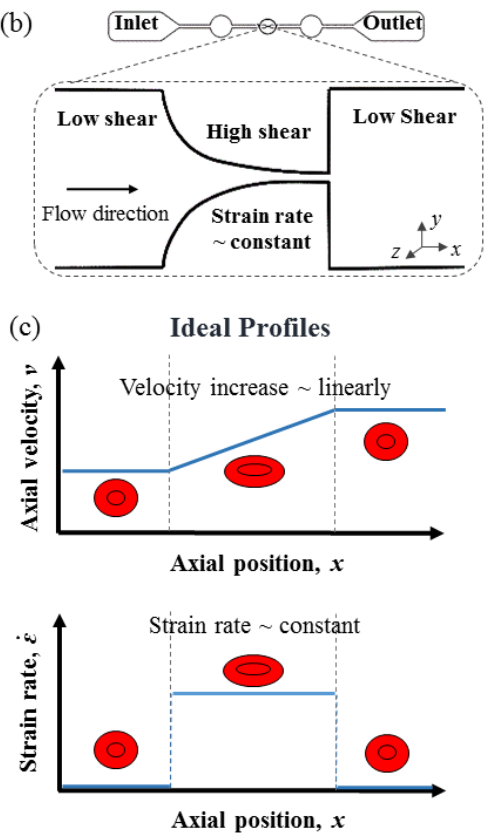

Figure 3.5. Microfluidic studies for the haemocompatibility of the RBCs in contact with MNPs. (a) schematic view of the experimental setup; (b) microchannel device geometry with the zoom of the hyperbolic channel; (c) graphical representation of the fluid-induced conditions profiles that occur in the hyperbolic channel, which has proven to be a more efficient way for the determination of the DI of the RBCs. Reprinted from [1]. Copyright @ 2016, with permission from Springer Nature.

Figure 3.6 shows a healthy RBC flowing along the axial position, $x$, of the microchannel with a hyperbolic-shaped contraction followed by a sudden expansion, which was subdivided in four sections to assess the DI evolution of the RBCs along the microchannel.

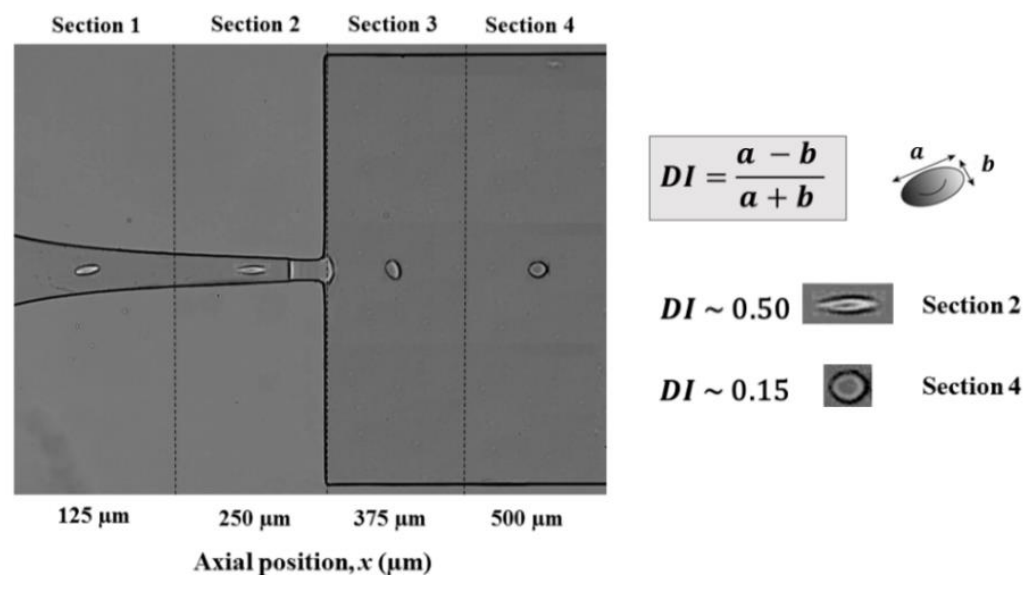

Figure 3.6. Representation of a healthy RBC flowing along the axial position of a microchannel with a hyperbolic-shaped contraction followed by a sudden expansion, subdivided in four sections with equal distances between them $(125 \mu \mathrm{m})$. This approach allows the assessment of the RBCs' DIs, as an indicator of the physiological effect of MNPs on the RBCs membranes. Furthermore, the DI equation is represented, as well as the typical values found for a healthy RBC subjected to a hyperbolic-shaped contraction (section 2) and to a recuperation section (section 4), under a controlled homogeneous extensional flow field. Reprinted from [1]. Copyright $\odot$ 2016, with permission from Springer Nature. 
Additionally, this figure shows the typical DI values found for healthy RBCs when exposed to a hyperbolic contraction (section $2, \sim 0.50$ ) followed by sudden expansion region (section 4 , $\sim 0.15)$, under a controlled homogeneous extensional flow field $[14,15]$.

Using the set of data listed in Table 3.1, the DIs of the RBCs (control versus MNPs) were measured flowing along the centreline of the microchannel that was subdivided in four sections (cf. Figure 3.6). The results are displayed in Figure 3.7. Furthermore, in order to compare the deformability behaviour of the tested samples, the average shear rate and particle Reynolds number $\left(R e_{p}\right)$ were calculated for the four different sections represented in Figure 3.6 and listed in Table 3.5. The average or pseudo shear rate is given by equation (3.4):

$$
\bar{\gamma}=\frac{U}{D_{h}}
$$

where $U$ is the mean velocity of the blood cells obtained at each region and $D_{h}$ is the hydraulic diameter of each microchannel section [34]. Additionally, the particle Reynolds number $\left(R e_{p}\right)$ was defined as equation (3.5):

$$
R e_{p}=\frac{\rho U D_{R B C}}{\mu}
$$

where $D_{R B C}$ is the diameter of the human RBC at rest, and $\rho$ the density of the Dextran 40 solution and $\mu$ the shear viscosity of the same solution [15]. Table 3.5 shows the mean \pm standard deviation values calculated for the $R e_{p}$ and average shear rate along the microchannel.

Table 3.5. Flow characteristics obtained for the four sections of the microchannel in the haemodynamic study. Reprinted from [1]. Copyright ( 2 2016, with permission from Springer Nature

\begin{tabular}{lcccc}
\hline Flow characteristics & Section 1 & Section 2 & Section 3 & Section 4 \\
\hline Particle Reynolds number & $0.08 \pm 0.01$ & $0.29 \pm 0.02$ & $0.05 \pm 0.01$ & $0.02 \pm 0.00$ \\
Average shear rate $\left(\mathrm{s}^{-1}\right)$ & $990.03 \pm 141.95$ & $5966.77 \pm 482.56$ & $505.02 \pm 35.99$ & $215.13 \pm 26.29$ \\
\hline
\end{tabular}

The $R e_{p}$ is a useful tool to assess the behaviour of the flowing cells suspended in the working fluid, taking into account the inertial and viscous forces that occur through the interactions of the blood cells with the fluid. The results listed in Table 3.5 show that this interaction is higher in section 2, where the average shear rate is also higher, and decreases in the recuperation sections (section 3 and 4), where the average shear rate becomes lower. Note 
that in microfluidic channels with a low aspect ratio $h / w$ (where $h$ refers to the depth $(20 \mu \mathrm{m})$ and $w$ refers to the width $(20-400 \mu \mathrm{m})$ of the microchannel), as those presented in our study, the RBC deformability is strongly affected by both extensional and shear flow conditions. Moreover, since the shear stress is usually reported in literature for the in vivo physiological flow conditions, the average shear rate was assessed and compared with values obtained in human vascular systems [35, 36].
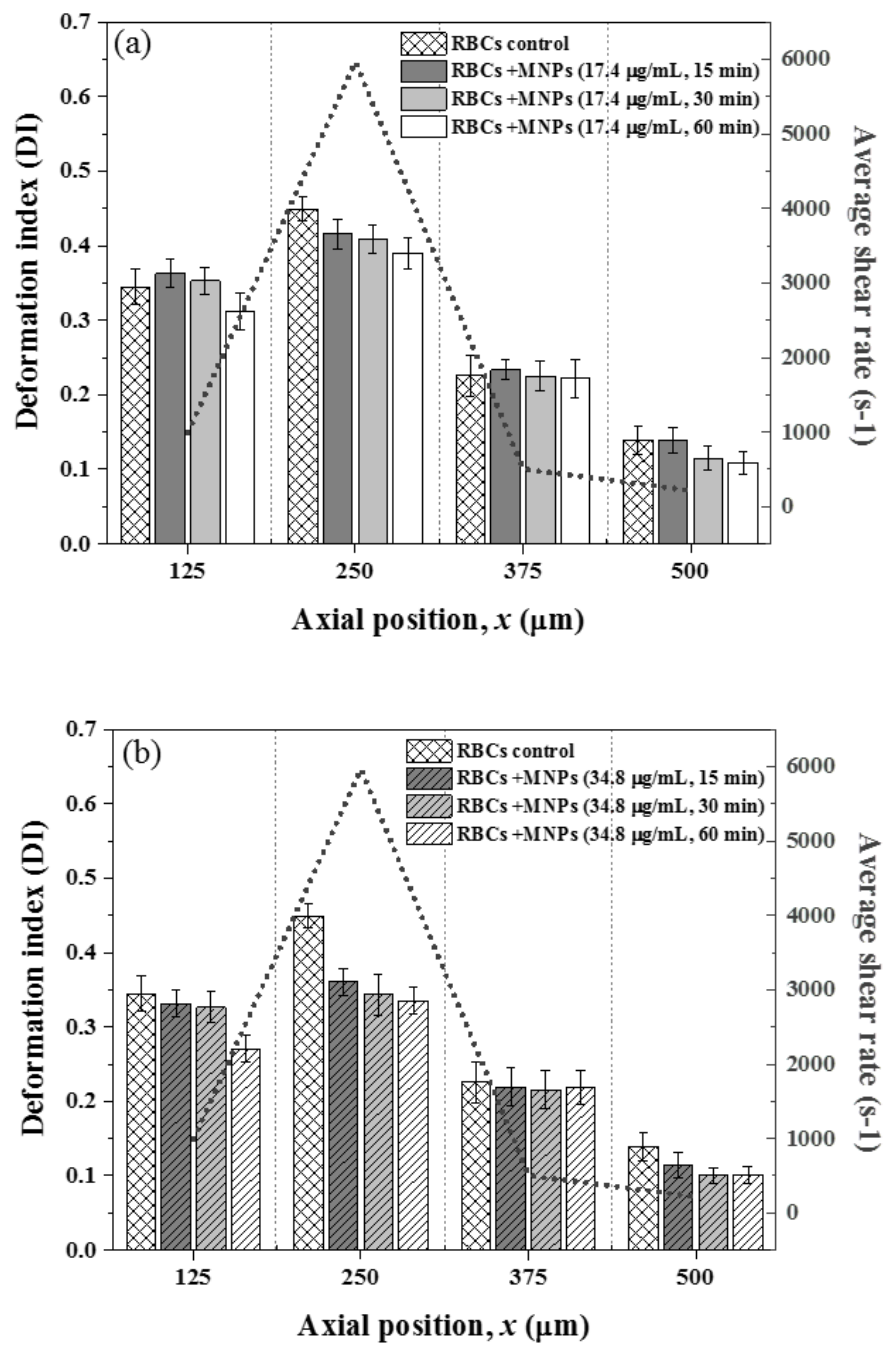

Figure 3.7. Deformation index of blood cells flowing along the axial position of the hyperbolic channel, as well as the average shear rate at each hyperbolic region: (a) control RBCs versus RBCs in contact with MNPs at a final concentration of $17.4 \mu \mathrm{g} \mathrm{Fe}_{04} \mathrm{~mL}^{-1}$ during 15,30 and $60 \mathrm{~min}$; (b) control

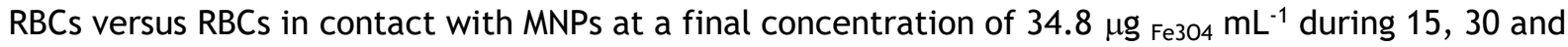
60 min. Error bars show a 95\% confidence interval $(n=56$ blood cells). Reprinted from [1]. Copyright (c) 2016, with permission from Springer Nature.

In addition, the low standard deviation obtained between all the samples along the microchannel sections for the measured $R e_{p}$, shows the maintenance of the same flow conditions, which is crucial for the further comparison between the DI results. As expected, the maximum DI obtained with all tested RBCs samples were measured in the hyperbolic 
contraction region, where the width is minimum (section 2, axial position $250 \mu \mathrm{m}$ ) and the average shear rate is higher $\left(\sim 6000 \mathrm{~s}^{-1}\right)$. Indeed, this value of shear rate is very close to that of the arterioles in the human vascular system $\left(\sim 8000 \mathrm{~s}^{-1}\right)[35,36]$ and thus, ideal to test the DI of RBCs at mimicked microvascular conditions. Overall, the higher DI, in this section 2 , was measured for the RBCs control (not exposed to MNPs), reaching a DI of $0.45 \pm 0.02$. It is worth mentioning that this result is in good agreement with the results found by Lee, et al. [14] for healthy RBCs submitted to higher shear rates $(0.42$ to 0.60 , for 0.6 to $9.0 \mathrm{~Pa}$ of extensional stress).

The DI results for the RBCs samples in contact with MNPs show that the incubation time influences directly the deformability of those blood cells. Hence, the DI results allow to conclude that the increase of the incubation time between RBCs and MNPs, lead to the decrease in $\mathrm{DI}$, or in other words, to the increase of rigidity of those cells. This observation is even more obvious for the sample with the higher content of MNPs and exposure time

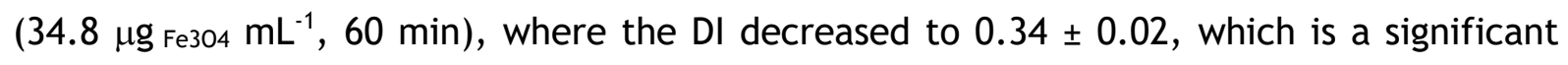
difference when compared with the control DI $(0.45 \pm 0.02)$.

The rigidity promoted by the MNPs into the RBCs membranes is also observed in the recuperation section (section 4, axial position $500 \mu \mathrm{m}$ ), where the average shear rate is minimum $\left(\sim 215 \mathrm{~s}^{-1}\right)$. In this section 4 , and comparing the results obtained between the control sample and the samples of RBCs that were placed in contact with the MNPs, it can be observed that RBCs incubated in the presence of MNPs become more rigid than the control RBCs, even when they are almost at rest. In the latter case, the control RBCs had ellipsoid shapes due to applied shear flow, whereas RBCs in contact with MNPs had geometries closer to a circular shape which corresponds to the behaviour of rigid cells.

Recently, Lin, et al. [32] were able to measure the RBCs DI after the blood cells have been in contact with nanodiamond nanoparticles at several shear stress, using a laser aggregometer-deformometer device. This study has shown that large nanoparticles $(100 \mathrm{~nm}$ average size) in contact with RBCs promote the reduction of the RBCs deformability, or the increase of their rigidity. Furthermore, using a confocal microscope and observing the blood samples in contact with the nanoparticles, the authors concluded that large nanodiamonds were located around the external environment of the RBCs, whereas smaller nanodiamonds ( $5 \mathrm{~nm}$ average size) penetrated inside the RBCs. As a result, it was established in this study that the deformability of RBCs decreases significantly for higher concentration of large nanodiamonds. This effect was ascribed to changes in the membranes of RBCs, probably caused by modifications in the structure and activity of their proteins, which may also lead to variations in the oxygenation-deoxygenation dynamics. More recently, similar conclusions 
were obtained by Curtis, et al. [37] through numerical simulations with hydrophilic nanoparticles with diameters ranging between 1 and $25 \mathrm{~nm}$, in a bilayer membrane (such as the RBCs membranes). The results have shown that hydrophilic nanoparticles with diameters greater than $2 \mathrm{~nm}$ become wrapped (the lipid head groups of a membrane form a vesicle around the nanoparticle), while smaller nanoparticles became embedded in the bilayer surface, penetrating into the membrane with minimal disruption to the structure. It is noteworthy that none of these studies can be directly correlated with the deformability results obtained in this work, and thus proving that the DI changes observed are due to the MNPs wrap and uptake by the blood cells membranes. This direct correlation cannot be done since the size, shape and composition also plays an important role in this cellular uptake. Therefore, further studies are required to clarify the complex interaction MNPs-RBCs. However, this work highlights the accuracy of the proposed microfluidic method, as a new and accurate tool to investigate the haemocompatibility of MNPs, where other conventional haematological methods, such as haemolysis tests, cannot show any specific alteration.

\subsubsection{Conclusions}

MNPs for theranostic applications were synthesized, characterized and tested for magnetic hyperthermia and MRI. The different heating behaviour of the synthesized MNPs shows the importance of the temperature as a synthesis parameter that allows the growth of the magnetic core and subsequently allows the increment of the saturation magnetization, while the nanoparticle size remains in the single domain region with superparamagnetic behaviour. As result, the iron oxide nanoparticles were produced through a cost efficient and industry friendly scalable procedure, proven to be suitable for theranostics application. Nevertheless, in order to be suitable for biomedical applications, the synthesized MNPs should be haematological safe. Thus, and since there is a lack of studies in literature regarding this issue, a high-sensitivity microfluidic tool was tested to gain insights into the complex nanoparticle-RBCs membrane interaction. This novel toxicological and haematological concept was based on the proven fact that the natural deformability of the RBCs can be used as biophysical marker to assess haematological pathologies or disorders. Hence, for the first time, it is presented a microfluidic tool capable to evaluate with high accuracy the impact of multifunctional nanoparticles designed for theranostic applications in contact with RBCs, by using the proved extensional flow approach to measure with high accuracy the RBCs' DIs. 
The results obtained in this section suggest that magnetite nanoparticles, with diameter around $18 \mathrm{~nm}$, are suitable for biomedical applications without showing haemolytic effect in the blood samples, even at the higher content of nanoparticles tested $\left(34.8 \mu \mathrm{g} \mathrm{Fe} 304 \mathrm{~mL}^{-1}\right)$. On the other hand, the applied microfluidic tool shows that small MNPs can influence the rigidity of RBCs in a time dependent manner, where other and more traditional biocompatibility tests (such as the haemolysis analysis) do not show any influence. Moreover, it demonstrates that this influence is directly related to the concentration of MNPs in the blood fluid. In fact, the tested microfluidic tool has shown a higher sensitivity to detect small changes in the RBC deformability, which is unreachable using conventional techniques. Due to this unique ability, this is the first experimental test reported in the literature that subscribe the numerical work performed by Curtis, et al. [37], which refers to the uptake of the surrounding MNPs by the RBC membranes, as the main reason for the increasing rigidity observed in the RBCs. In this recently numerical study, it was also predicted a dependency on the MNP size, shape and composition with the RBC membrane interaction.

It should be noticed that to gain a further understanding of the haemo-rheological and biological interaction between the MNPs and RBCs, other studies should be performed to complement this one. Nevertheless, the presented haematological and toxicological results confirm that it is highly desirable that blood properties, including the effect of the MNPs on the deformability of RBCs, and other rheological properties, should be controlled and tested together with the traditional biocompatible tests. These new achievements and the effective understanding of the interaction between MNPs and the blood cells, can help to promote the use of nanomedicine to the next level. For instance, the MNPs-RBCs interactions revealed in this study, can be in the future investigated as a new approach to deliver MNPs directly into the tumours, by using the RBCs as biocarriers that naturally escape from the mononuclear phagocyte system. Hence, the proposed microfluidic methodology represents a new concept for the multidisciplinary nanomedicine field with proven benefits to evaluate the haemocompatibility of MNPs that are being developed for clinical applications. 


\subsection{Thermal infrared image processing to assess heat generated by magnetic nanoparticles for hyperthermia applications}

Alongside with the lack of haemocompatibility tests, the poor heating efficiency of most MNPs [38] and the imprecise experimental determination of the temperature field during hyperthermia, are still major drawbacks of magnetic hyperthermia. Indeed, the imprecise quantification of the heat generated by MNPs under an AMF, can result either in insufficient heating to destroy cancer cells or in the over-heating with collateral damage of the healthy ones [39]. Usually, the temperature field is measured during the treatment using optic fibre probes. Nevertheless, these captors cannot be precisely implanted in the tumour tissues and they only give local temperature information [39].

Infrared thermography, based on the physical phenomenon that all bodies at temperatures above absolute zero generate heat by radiation in the infrared portion of the electromagnetic spectrum [40], can overcome the weaknesses of the optic fibre probes. This methodology allows the creation of a thermal map emitted from the body (both healthy and tumour tissues), as well as from the agglomerated MNPs.

In this section, an infrared thermography device was used to assess the heat released by different sets of MNPs when subjected to a home-made AMF apparatus. For this purpose, the thermal images acquired by the infrared camera were calibrated and processed using MATLAB. The study presented in this section shows the ability to use this methodology as a screening tool to evaluate the potentiality of novel synthesized MNPs to be further applied in hyperthermia.

\subsubsection{Materials and methods}

\subsubsection{Chemicals}

Ammonium hydroxide solution $\left(\mathrm{NH}_{4} \mathrm{OH}, 25 \mathrm{wt} \%\right.$ in $\left.\mathrm{H}_{2} \mathrm{O}\right)$ was purchased from Merck (NJ, USA). Iron (II) chloride tetrahydrate $\left(\mathrm{FeCl}_{2} .4 \mathrm{H}_{2} \mathrm{O}, 99 \%\right)$, Dextran $40\left(\left(\mathrm{C}_{6} \mathrm{H}_{10} \mathrm{O}_{5}\right)_{n}\right)$ and ethanol absolute $\left(\mathrm{C}_{2} \mathrm{H}_{6} \mathrm{O}\right.$, 99.8\%) were purchase from Sigma-Aldrich (MO, USA). Iron (III) chloride hexahydrate $\left(\mathrm{FeCl}_{3} .6 \mathrm{H}_{2} \mathrm{O}, 97 \%\right)$ was supplied by Panreac (Barcelona, Spain). All chemicals were of analytical grade and used as received without further purification. All aqueous solutions were prepared in deionized water. 


\subsubsection{Synthesis of magnetic nanoparticles}

Samples MAG30 and MAG75 were synthesized through the co-precipitation method by using the same procedure described in section 3.2.1.2, but using as synthesis temperatures 30 and $75{ }^{\circ} \mathrm{C}$, respectively. In addition, a set of bare MNP was synthesized in a similar way at room temperature (MAG25). In this case, after co-precipitation, MAG25 was transferred to a teflon-lined stainless-steel autoclave $(125 \mathrm{~mL})$ and maintained at $180{ }^{\circ} \mathrm{C}$ under its autogenous pressure during $24 \mathrm{~h}$. The resulting MAG25 sample was further coated with graphene oxide $(\mathrm{GO})^{1}$ by using a simple electrostatic self-assembly process (MAG25@GO), with a method similar to that described by Wei et al. (2012) [41].

\subsubsection{Physicochemical characterization of MNPs}

The crystallite phases and core sizes of the synthesized MNPs (MAG25, MAG30, MAG75 and MAG25@GO) were analysed by X-ray powder diffraction (XRD, PAN'alytical X'Pert PRO, Netherlands) by using the Bragg's law.

\subsubsection{Experimental setup}

The adequate heat release promoted by MNPs when subjected to an AMF is one of the most important key-points for the success of magnetic hyperthermia. Heat release is directly dependent on three main parameters: (1) the crystalline microstructure of the MNPs, (2) the intensity of the AMF and (3) the method for temperature acquisition.

In order to study these three features, the experimental work was divided in two main experimental setups: (i) calibration of the infrared camera and (ii) thermal imaging acquisition of MNPs under an AMF.

\section{(i) Calibration of the infrared camera}

For the calibration of the infrared camera, the surface of the containers (beakers) and the solutions used for the dispersion of MNPs were assessed. As containers, two beakers of $100 \mathrm{~mL}$ fabricated in two different materials, borosilicate glass and polypropylene (PP) polymer with the same dimensions, $5 \mathrm{~cm} \times 7 \mathrm{~cm}$ (outer diameter $\times$ height), were used. Each beaker was filled with $100 \mathrm{~mL}$ of the working fluid, deionized water and glycerine $70 \%$, respectively. These containers were further heated at increments of

${ }^{1} \mathrm{GO}$ was kindly supplied by Luisa Pastrana-Martínez, Associate Laboratory LSRE-LCM, Faculty of Engineering, 
$5{ }^{\circ} \mathrm{C}$ between 20 and $50{ }^{\circ} \mathrm{C}$, by using a magnetic heating stirrer plate with an integrated thermocouple (C-Mag HS7, IKA, Germany). The temperature increase was confirmed by using a second individual thermocouple. During this temperature increase, several thermal images were acquired with the infrared camera (E30, FLIR, USA) placed at $50 \mathrm{~cm}$ of the experimental setup and transferred to a PC for posterior analysis using MATLAB (R2015a, Mathworks, USA). The experimental tests and the parameters considered in the infrared camera calibration are shown in Table 3.6.

Table 3.6. Calibration experimental tests and the parameters considered in the infrared camera. Reprinted from [2]. Copyright @ 2015, with permission from Springer Nature

\begin{tabular}{cccc}
\hline Calibration test & Object material & Working fluid & Temperature range \\
\hline 1 & Glass & Deionized water & $20-50^{\circ} \mathrm{C}$ \\
2 & PP & Deionized water & $20-50^{\circ} \mathrm{C}$ \\
3 & PP & Glycerine $70 \%$ & $20-50^{\circ} \mathrm{C}$ \\
\hline
\end{tabular}

(ii) Thermal imaging acquisition of MNPs under an AMF

After the calibration of the thermography method, the experimental setup for the assessment of the heat released by the synthesized MNPs was assembled as shown in Figure 3.8. The AMF apparatus used in this work was a home-made non-adiabatic device, designed to work at a maximum intensity of $1.10 \mathrm{kA} \mathrm{m}^{-1}$ and with a frequency of $200 \mathrm{kHz}$.

To investigate the influence of the working fluids in the heat released by the MNPs under an $A M F$, different fluids were considered to perform the infrared camera calibration (deionized water and glycerine $70 \%$ ). The infrared camera was placed at $50 \mathrm{~cm}$ of the samples, which were involved by the coil-shaped heating station (detail shown in Figure 3.8), generating the AMF.

The experimental assays and the parameters considered to assess the heat released by the different synthesized MNPs (MAG30, MAG50 and MAG25@GO) are shown in Table 3.7.

Additionally, the thermal imaging assays shown in Table 3.7 were repeated with controls (working fluids without MNPs). These controls allowed the correction of the heat generated by the MNPs by removing the heat generated by the coil (Joule heating) during the experimental tests, since was employed as a non-adiabatic AC magnetic apparatus. Periodic infrared images were taken in all thermal image assays at 0, 60, 120, 300 and $600 \mathrm{sec}$, recorded in the format.JPEG and further processed in MATLAB. 


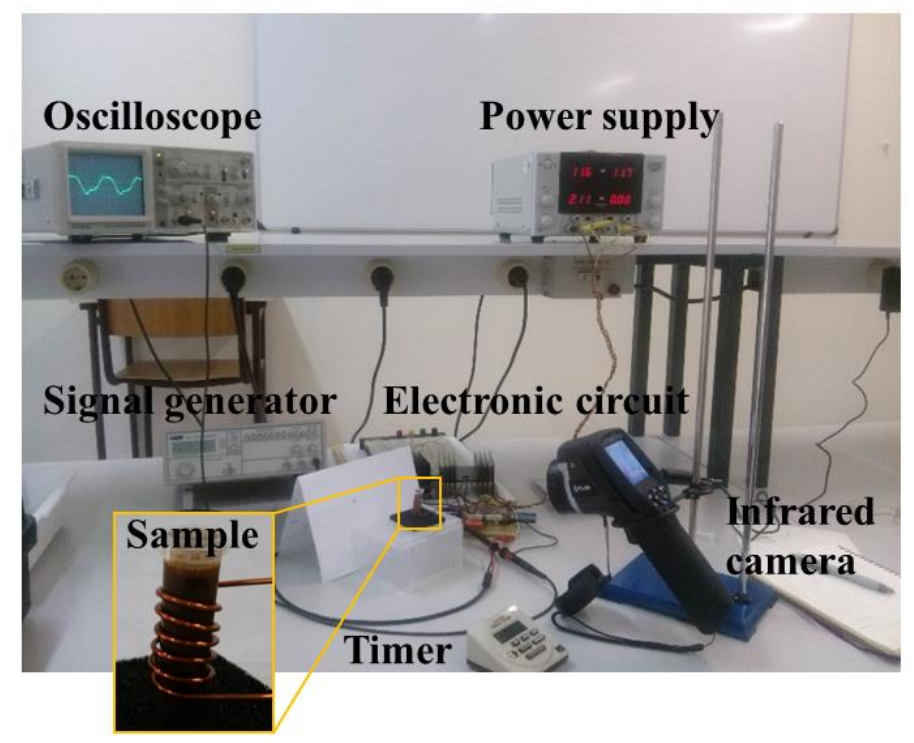

Figure 3.8. Experimental setup for the assessment of heat generated by MNPs under an AMF, monitored by the infrared camera (E30, FLIR, USA). Copyright $\odot$ 2015, with permission from Springer Nature.

Table 3.7. Experimental assays and the parameters considered to assess the heat generated by the different MNPs under an AMF. Copyright (c) 2015, with permission from Springer Nature

\begin{tabular}{ccccc}
\hline Assay & Carrier Fluid & $\begin{array}{c}\text { Fluid density } \\
\left(\mathrm{g} \mathrm{cm}^{-3}\right)\end{array}$ & $\begin{array}{c}\text { MNPs concentration } \\
\left(\mathrm{mg} \mathrm{mL}^{-1}\right)\end{array}$ & $\begin{array}{c}\text { Magnetic field } \\
\left(\mathrm{kA} \mathrm{m}^{-1} \text { @ kHz) }\right.\end{array}$ \\
\hline 1 & Deionized water & 1.00 & 5.0 & 1.10 @ 200 \\
2 & Glycerine $70 \%$ & 1.18 & 5.0 & 1.10 @ 200 \\
3 & Glycerine 70\% & 1.18 & 5.0 & $0.80 @ 250$ \\
\hline
\end{tabular}

\subsubsection{Thermal infrared image processing in $M A T L A B$}

The thermal infrared images acquired in the calibration and experimental tests were recorded in the format .JPEG with $160 \times 120$ pixels. Then, the images were transferred to a PC and converted into MATLAB readable files with the extension. MAT by using the software ThermaCAM Researcher (trial version 2.10 Pro, FLIR, USA). This new format allows the use of the original information captured by the infrared camera, where each pixel corresponds to a temperature captured in Kelvin (K).

(i) Calibration of the infrared camera

The thermal images acquired during the calibration tests with extension .MAT, ands matrix $120 \times 160$ corresponding to temperatures in $\mathrm{K}$, were converted to Celsius degrees $\left({ }^{\circ} \mathrm{C}\right)$ by using the following code [42]:

name='Ir_0323'; \%Name of the thermal image 


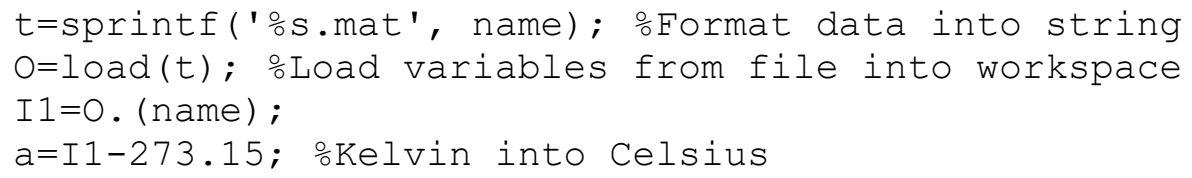

After the creation of the new matrix (a), with the temperature in ${ }^{\circ} \mathrm{C} /$ pixel, the APP Image Viewer of MATLAB (version R2015a) was used to open $a$ with the following command: File $>$ Import from workspace $>a>0 k$. Then, by selecting the icon contrast, each image was displayed, zoomed to $200 \%$ and the desired part of the image containing the heated fluid was selected using the icon crop image. The region of interest was further transferred to the workspace of MATLAB with the menu File > Export to Workspace > Image variable name $>I$. Finally, calling the $(I)$, the mean temperature of this matrix was calculated as following:

$$
>>\text { mean (mean (I)) }
$$

\section{(ii) Heat released by the MNPs under an AMF}

In a similar way to the image processing described above for the calibration of the infrared camera, the infrared images obtained from the thermal imaging assays (assays 1, 2 and 3, as shown on Table 3.7), were transferred to MATLAB with the extension .MAT. In a similar way, the temperature displayed in $\mathrm{K}$ was converted to a matrix in ${ }^{\circ} \mathrm{C}$. The new matrix (a), was open in the APP Image Viewer of MATLAB (version R2015a), using the following command: File > Import from workspace $>a>o k$. Then, by selecting the icon contrast the image was displayed, zoomed to $800 \%$ and the desired parts of the image $\left(R_{1}\right.$, $R_{2}, R_{3}$ and $R_{4}$ ), containing the fluid heated by the MNPs under the AMF (within the loops of the coil), were selected with the icon crop image, as shown in Figure 3.9.

a)

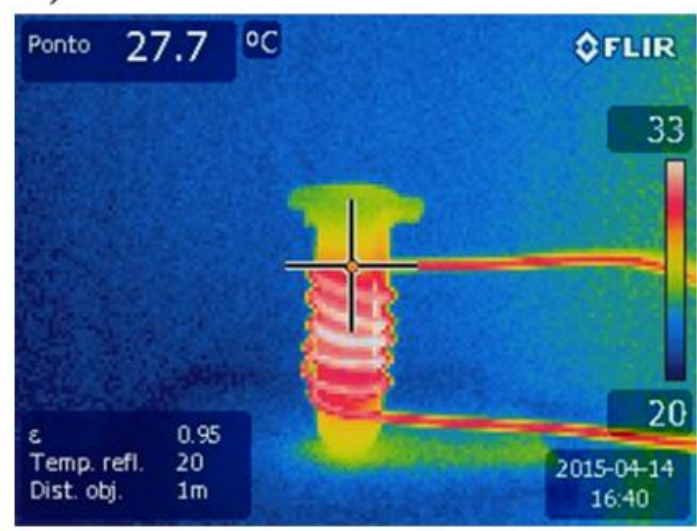

b)

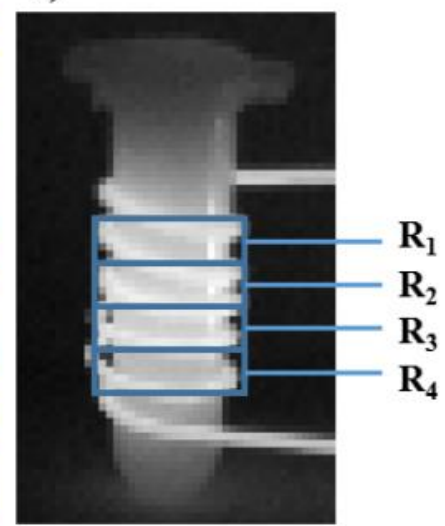

Figure 3.9. Representation of an infrared image captured during the heat release of MNPs under an AMF: (a) thermal image obtained as .JPEG with the infrared camera; (b) thermal imaging processing showing the four regions of interest $\left(R_{1}, R_{2}, R_{3}\right.$ and $\left.R_{4}\right)$. Copyright $\odot 2015$, with permission from Springer Nature. 


\subsubsection{Results and discussion}

\subsubsection{Physicochemical characterization of MNPs}

The synthesized MNPs were characterized by XRD, as summarized in Table 3.8.

Table 3.8. Physicochemical properties of the synthesized nanoparticles: crystallite size calculated from XRD $\left(\mathrm{d}_{\mathrm{XRD}}\right)$, lattice constant and crystalline core phase obtained by XRD analysis. Copyright $\odot$ 2015, with permission from Springer Nature

\begin{tabular}{ccccc}
\hline Sample & $\begin{array}{c}\text { Synthesis } \\
\text { temperature } \\
\left({ }^{\circ} \mathrm{C}\right)\end{array}$ & $\begin{array}{c}\mathrm{d}_{\mathrm{XRD}} \\
(\mathrm{nm})\end{array}$ & $\begin{array}{c}\text { Lattice } \\
\text { constant, } a \\
(\AA)\end{array}$ & $\begin{array}{c}\text { Core phases } \\
(\mathrm{v} / \mathrm{v})\end{array}$ \\
\hline MAG30 & $30{ }^{\circ} \mathrm{C}$ & $12.80 \pm 0.20$ & 8.3652 & $100 \%$ Magnetite \\
MAG75 & $75{ }^{\circ} \mathrm{C}$ & $32.20 \pm 0.30$ & 8.3770 & $100 \%$ Magnetite \\
MAG25@GO & $180^{\circ} \mathrm{C}$ & $63.05 \pm 1.00$ & 5.0345 & $49.9 \%$ Hematite \\
& & $30.04 \pm 0.30$ & 8.3683 & $50.1 \%$ Magnetite \\
\hline
\end{tabular}

Table 3.8 puts in evidence the influence of the synthesis temperature on the growth of the crystallite size of the synthesized MNPs, as already observed in the study made in section 3.2. As observed before, the increase of the synthesis temperature allows the growth of the crystallite core of MNPs. The XRD analysis also shows that the hydrothermal treatment at $180{ }^{\circ} \mathrm{C}$ during $24 \mathrm{~h}$ of MAG25@GO, leaded to an additional growth of the magnetic core, as well as to a partial oxidation to hematite (as observed in the lattice constant, $a$ ). It is worth mentioning that hematite is a form of iron oxide with less magnetic saturation [43] and therefore, with lower heating power than magnetite under the same AMF.

\subsubsection{Calibration of Infrared camera}

As previously shown in Table 3.6, the calibration of the infrared camera was performed through three tests. On these tests, different materials of the containers and different working solutions were taken into account to determine their influence in the acquisition of the thermal images, as well as to obtain the correlation between inner and outer temperatures.

Overall, as shown in Table 3.9, the local temperature given by the pointer of the infrared camera (FLIR) is in good agreement with the mean temperature obtained from the region of interest obtained by using MATLAB (MAT). The good agreement between the local and mean temperatures of the region of interest can be explained by the magnetic stirring of the liquid 
samples that allowed the homogenization of solutions, and thus the homogenization of the heating transfer along the thermal tests.

Table 3.9. Temperature results obtained with the TC (Thermocouple temperature), FLIR (Infrared camera pointer temperature) and MAT (mean temperature of the region of interest obtained in MATLAB) during calibration tests. Copyright $\odot$ 2015, with permission from Springer Nature

\begin{tabular}{|c|c|c|c|c|c|c|c|c|}
\hline \multicolumn{3}{|c|}{$\begin{array}{l}\text { Calibration test } 1 \\
\text { (Glass, DW) }\end{array}$} & \multicolumn{3}{|c|}{$\begin{array}{l}\text { Calibration test } 2 \\
\text { (PP, DW) }\end{array}$} & \multicolumn{3}{|c|}{$\begin{array}{l}\text { Calibration test } 3 \\
\text { (PP, Glycerine } 70 \%)\end{array}$} \\
\hline $\begin{array}{l}\mathrm{TC} \\
\left({ }^{\circ} \mathrm{C}\right)\end{array}$ & $\begin{array}{l}\text { FLIR } \\
\left({ }^{\circ} \mathrm{C}\right)\end{array}$ & $\begin{array}{l}\text { MAT } \\
\left({ }^{\circ} \mathrm{C}\right)\end{array}$ & $\begin{array}{l}\text { TC } \\
\left({ }^{\circ} \mathrm{C}\right)\end{array}$ & $\begin{array}{l}\text { FLIR } \\
\left({ }^{\circ} \mathrm{C}\right)\end{array}$ & $\begin{array}{l}\text { MAT } \\
\left({ }^{\circ} \mathrm{C}\right)\end{array}$ & $\begin{array}{l}\text { TC } \\
\left({ }^{\circ} \mathrm{C}\right)\end{array}$ & $\begin{array}{l}\text { FLIR } \\
\left({ }^{\circ} \mathrm{C}\right)\end{array}$ & $\begin{array}{l}\text { MAT } \\
\left({ }^{\circ} \mathrm{C}\right)\end{array}$ \\
\hline 19.3 & 18.4 & 18.7 & 20.0 & 19.6 & 20.1 & 21.7 & 21.7 & 21.7 \\
\hline 25.0 & 24.0 & 24.1 & 25.0 & 24.2 & 24.4 & 25.0 & 24.8 & 24.9 \\
\hline 30.0 & 28.3 & 28.5 & 30.0 & 29.1 & 29.6 & 30.0 & 29.5 & 29.5 \\
\hline 35.0 & 32.9 & 32.8 & 35.0 & 34.7 & 34.7 & 35.0 & 33.8 & 33.8 \\
\hline 40.0 & 37.2 & 37.1 & 40.0 & 39.3 & 39.4 & 40.0 & 38.9 & 38.7 \\
\hline 45.0 & 41.8 & 41.7 & 45.0 & 46.1 & 46.2 & 45.0 & 43.3 & 43.3 \\
\hline 50.0 & 46.3 & 46.1 & 50.0 & 49.8 & 50.1 & 50.0 & 48.8 & 48.8 \\
\hline
\end{tabular}

Additionally, Table 3.9 also shows a discrepancy between the temperatures recorded by the thermocouple (TC) (placed inside the fluids) and the FLIR temperatures (obtained at the outer surface of the beakers). Thus, calibration curves and linear fits (data not shown) between the temperatures acquired by the TC and the MALTAB analysis, for the three calibration tests, were determined. These correlations allowed the true acquisition of the heat release caused by the MNPs under the AMF tests. By using equation 3.6, the relative errors from these tests were also calculated:

Relative error $=\frac{\sum_{i=0}^{n}(\text { Inlet temperature (Thermocouple) }- \text { Outlet temperature (MATLAB)) }}{n}$

where $n$ is the total number of measures and $i$ the measurement number.

As a result, the relative error was found to be $2.19^{\circ} \mathrm{C}$ for the calibration test $1,0.23{ }^{\circ} \mathrm{C}$ for the calibration test 2 , while for the calibration test 3 , an error of $0.86{ }^{\circ} \mathrm{C}$ was obtained. These results show that the use of polypropylene polymer (PP) containers brings a more accurate thermal measurement between the inner and the outer surface of the materials. Therefore, PP was found to be a better choice than glass in this type of experiments. Interestingly, the difference in the results obtained between the use of PP and glass cannot be explained by the thermal conductivity of the materials. In fact, borosilicate glass has a higher thermal conductivity than PP. A possible explanation for the results can be attributed to some glass reflection that was experienced during the acquisition of the thermal images. 


\subsubsection{Heat released by MNPs under an AMF}

One of the crucial steps to perform hyperthermia tests is the colloidal stabilization of the MNPs within the working fluid, also known as ferrofluid. Therefore, in order to compare the influence of two working fluids with different densities (deionized water and glycerin 70\%), two thermal imaging assays (thermal assays 1 and 2, respectively) were performed using an AMF with $1.10 \mathrm{kA} \mathrm{m}^{-1}$ and $200 \mathrm{kHz}$, during $600 \mathrm{sec}$ (cf. Table 3.7). Additionally, thermal assay 3, was performed to check the influence of the frequency increase $(250 \mathrm{kHz})$ in the heat generated by the MNPs.

As shown in Figure 3.10, the thermal assay 2, performed with glycerine $70 \%$ as working fluid and an AMF of $1.10 \mathrm{kA} \mathrm{m}^{-1}$ and $200 \mathrm{kHz}$, was the one that provided the best conditions to generate heat from the synthesized MNPs.

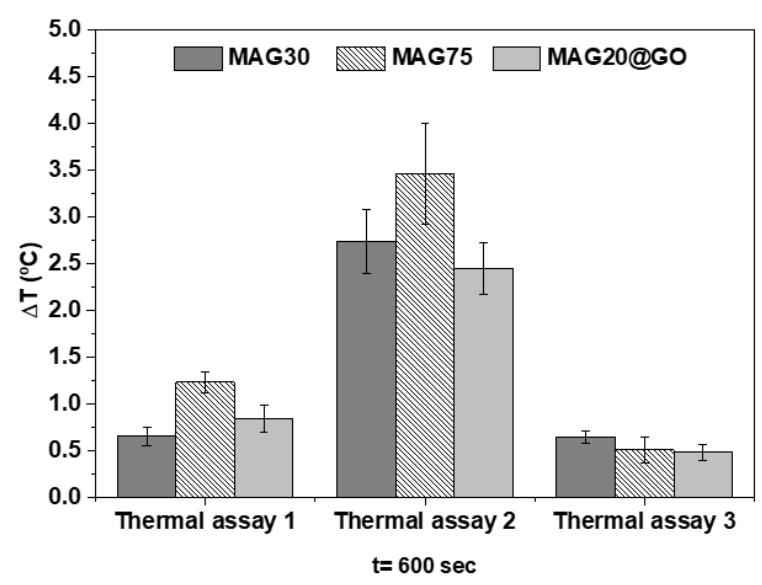

Figure 3.10. Comparison of the heat released by the different synthesized MNPs $\left(5 \mathrm{mg} \mathrm{mL}^{-1}\right)$, during three thermal assays. The mean temperature values are corrected by the respective calibration curves calculated during the calibration of infrared camera and normalized with the heat generated by the coil (blank tests). Error bars correspond to the standard deviation obtained from the four regions of interest $\left(R_{1}-R_{4}\right)$ in each thermal experiment. Copyright $\odot 2015$, with permission from Springer Nature.

Comparing the heating results of the thermal assays 1 and 2, performed at the same AMF conditions, it is also observed that the stabilization of the MNPs in the proper working fluid is a crucial step for the performance of hyperthermia tests. The importance of this stabilization is related to the homogenization of the ferrofluid under the AMF, which reach a maximum magnetic intensity within the coil. Without this stabilization, the MNPs tend to sediment in the bottom of the vials. This fact is especially critical when the AMF apparatus generate a maximum magnetic field intensity that is 20 times lower $(\sim 1.10 \mathrm{kA} / \mathrm{m}$, like in our case) than a commercial apparatus, where these hyperthermia tests are normally performed at around $20 \mathrm{kA} \mathrm{m}^{-1}$. Nevertheless, the home-made AC magnetic apparatus used in this work allowed, even with a very low AMF, a maximum temperature increase of 
$3.45 \pm 0.54{ }^{\circ} \mathrm{C}$ for sample MAG75, after $600 \mathrm{sec}$ under the AMF. In fact, this result is in good agreement with the literature, where it is reported that MNPs under the single-domain size and superparamagnetic properties increased their heating power as they grow in size [10].

For the case of the MNPs synthesized at $180{ }^{\circ} \mathrm{C}$ and coated with GO (MAG25@GO), the presence of almost $50 \%$ of hematite (with lower $M_{s}$ than magnetite) and higher core sizes (around $100 \mathrm{~nm}$ in total), puts these MNPs into the ferromagnetic materials. In fact, the ferromagnetic materials are considered in literature inappropriate materials to be used in biomedical applications, since these MNPs remain magnetized for a long period of time, even after removal of the applied magnetic field (high coercivity). Additionally, these materials released heat mainly by the hysteresis heating mechanism. Once again, these screening results obtained with a home-made $A C$ magnetic equipment are in good agreement with the characterization results and the literature in the field, which shows the ability to use this optical methodology to perform the assessment of the heat generated by MNPs under magnetic hyperthermia.

\subsubsection{Conclusions}

Hyperthermia is a promising therapeutic technique for the treatment of cancer, but the clinical successful application is intrinsically linked to the development of new nanomaterials and the improvement of new methodologies to determine the temperature during treatment. In this section, MNPs with different sizes were synthesized at different synthesis temperature $\left(30,75\right.$ and $\left.180^{\circ} \mathrm{C}\right)$, and their heating efficiency assessed through the use of a home-made AMF apparatus and an infrared camera. The acquired thermal images were transferred to a PC and the images processed in MATLAB. In order to correlate the inner and outer temperatures of different containers and working fluids, calibration tests were performed with deionized water and glycerine $70 \%$, as well as with different materials (glass and PP). These calibrations were used to correct and normalize the experimental data. The screening hyperthermia assays revealed that the MNPs synthesized by co-precipitation at $75^{\circ} \mathrm{C}$ (MAG75) were those providing higher heating power at the tested conditions. These results are related to the fact that this set of MNPs present single-domain size and superparamagnetic properties, which increase their heating power as they grow in size. Therefore, the main conclusion of this section is that the use of an infrared camera is a promising technique to assess the heat released by MNPs during magnetic hyperthermia, even when the AMF system is not able to create high magnetic fields, as in the case of the home-made AC magnetic apparatus used in this work. Moreover, this infrared methodology can be a very effective tool to assess the thermal map of 3D tissues to test MNPs. 


\section{References}

[1] R. O. Rodrigues, M. Bañobre-López, J. Gallo, P. B. Tavares, A. M. T. Silva, R. Lima and H. T. Gomes, Journal of Nanoparticle Research, 2016, 18, 1-17.

[2] R. O. Rodrigues, H. T. Gomes, R. Lima, A. M. T. Silva, P. J. S. Rodrigues, P. B. Tavares and J. M. R. S. Tavares, in Advances in Visual Computing: Lecture Notes in Computer Science, eds. G. Bebis, et al., Springer International Publishing, Cham, 2015, pp. 25-34.

[3] M. Mahmoudi, S. Sant, B. Wang, S. Laurent and T. Sen, Advanced Drug Delivery Reviews, 2011, 63, 24-46.

[4] J. Baumgartner, L. Bertinetti, M. Widdrat, A. M. Hirt and D. Faivre, PLoS ONE, 2013, 8, e57070.

[5] D. F. De Haas-Kock, J. Buijsen, M. Pijls-Johannesma, L. Lutgens, G. Lammering, G. A. van Mastrigt, D. K. De Ruysscher, P. Lambin and J. van der Zee, Cochrane Database of Systematic Reviews, 2009, CD006269.

[6] I. Hocaoglu, D. Asik, G. Ulusoy, C. Grandfils, I. Ojea-Jimenez, F. Rossi, A. Kiraz, N. Doğan and H. Y. Acar, Colloids and Surfaces B: Biointerfaces, 2015, 133, 198-207.

[7] M. Szekeres, E. Illés, C. Janko, K. Farkas, I. Y Tóth, D. Nesztor, I. Zupkó, I. Foldesi, C. Alexiou and E. Tombácz, Journal of Nanomedicine and Nanotechnology, 2015, 6, 1000252.

[8] Q. Wang, M. Shen, T. Zhao, Y. Xu, J. Lin, Y. Duan and H. Gu, Scientific reports, 2015, $5,7774$.

[9] K. Hayashi, M. Nakamura, W. Sakamoto, T. Yogo, H. Miki, S. Ozaki, M. Abe, T. Matsumoto and K. Ishimura, Theranostics, 2013, 3, 366-376.

[10] V. Zavisova, M. Koneracka, J. Kovac, M. Kubovcikova, I. Antal, P. Kopcansky, M. Bednarikova and M. Muckova, Journal of Magnetism and Magnetic Materials, 2015, 380, 85-89.

[11] I. P. Grudzinski, M. Bystrzejewski, M. A. Cywinska, A. Kosmider, M. Poplawska, A. Cieszanowski and A. Ostrowska, Journal of Nanoparticle Research, 2013, 15, 1835.

[12] J. Tan, A. Thomas and Y. Liu, Soft Matter, 2012, 8, 1934-1946.

[13] M. Abkarian, M. Faivre, R. Horton, K. Smistrup, C. A. Best-Popescu and H. A. Stone, Biomedical Materials, 2008, 3, 034011.

[14] S. S. Lee, Y. Yim, K. H. Ahn and S. J. Lee, Biomedical microdevices, 2009, 11, 10211027.

[15] T. Yaginuma, M. S. N. Oliveira, R. Lima, T. Ishikawa and T. Yamaguchi, Biomicrofluidics, 2013, 7, 054110.

[16] V. Faustino, D. Pinho, T. Yaginuma, R. Calhelha, I. F. R. Ferreira and R. Lima, BioChip Journal, 2014, 8, 42-47.

[17] M. S. N. Oliveira, M. A. Alves, F. T. Pinho and G. H. McKinley, Experiments in Fluids, 2007, 43, 437-451.

[18] R. Lima, S. Wada, S. Tanaka, M. Takeda, T. Ishikawa, K. Tsubota, Y. Imai and T. Yamaguchi, Biomedical microdevices, 2008, 10, 153-167.

[19] Z. A. Khorsand, W. H. Abd. Majid, M. E. Abrishami and R. Yousefi, Solid State Sciences, 2011, 13, 251-256. 
[20] J. C. Aphesteguy, G. V. Kurlyandskaya, J. P. de Celis, A. P. Safronov and N. N. Schegoleva, Materials Chemistry and Physics, 2015, 161, 243-249.

[21] Y. V. Kolen’ko, M. Bañobre-López, C. Rodríguez-Abreu, E. Carbó-Argibay, A. Sailsman, Y. Piñeiro-Redondo, M. F. Cerqueira, D. Y. Petrovykh, K. Kovnir, O. I. Lebedev and J. Rivas, The Journal of Physical Chemistry C, 2014, 118, 8691-8701.

[22] A.-H. Lu, E. L. Salabas and F. Schüth, Angewandte Chemie International Edition, 2007, 46, $1222-1244$.

[23] I. Obaidat, B. Issa and Y. Haik, Nanomaterials, 2015, 5, 63.

[24] A. E. Deatsch and B. A. Evans, Journal of Magnetism and Magnetic Materials, 2014, 354, 163-172.

[25] A. Hervault and N. T. K. Thanh, Nanoscale, 2014, 6, 11553-11573.

[26] I. Sharifi, H. Shokrollahi and S. Amiri, Journal of Magnetism and Magnetic Materials, 2012, 324, 903-915.

[27] P. Presa, Y. Luengo, M. Multigner, R. Costo, M. P. Morales, G. Rivero and A. Hernando, The Journal of Physical Chemistry C, 2012, 116, 25602-25610.

[28] K. C. Barick, S. Singh, D. Bahadur, M. A. Lawande, D. P. Patkar and P. A. Hassan, Journal of Colloid and Interface Science, 2014, 418, 120-125.

[29] J. S. Weinstein, C. G. Varallyay, E. Dosa, S. Gahramanov, B. Hamilton, W. D. Rooney, L. L. Muldoon and E. A. Neuwelt, Journal of Cerebral Blood Flow and Metabolism, 2010, 30, 15-35.

[30] J. Gallo, I. S. Alam, I. Lavdas, M. Wylezinska-Arridge, E. O. Aboagye and N. J. Long, Journal of Materials Chemistry B, 2014, 2, 868-876.

[31] A. Saraswathy, S. S. Nazeer, M. Jeevan, N. Nimi, S. Arumugam, V. S. Harikrishnan, P. R. Varma and R. S. Jayasree, Colloids and Surfaces B: Biointerfaces, 2014, 117, 216224.

[32] Y. C. Lin, L. W. Tsai, E. Perevedentseva, H. H. Chang, C. H. Lin, D. S. Sun, A. E. Lugovtsov, A. Priezzhev, J. Mona and C. L. Cheng, Journal of Biomedical Optics, 2012, 17, 101512.

[33] R. Rodrigues, D. Pinho, V. Faustino and R. Lima, Biomedical microdevices, 2015, 17, 19.

[34] K. Müller, D. A. Fedosov and G. Gompper, Scientific reports, 2014, 4, 4871.

[35] J. E. Sadler, Annual review of biochemistry, 1998, 67, 395-424.

[36] P. Vennemann, R. Lindken and J. Westerweel, Experiments in Fluids, 2007, 42, 495511.

[37] E. M. Curtis, A. H. Bahrami, T. R. Weikl and C. K. Hall, Nanoscale, 2015, 7, 1450514514.

[38] R. Di Corato, A. Espinosa, L. Lartigue, M. Tharaud, S. Chat, T. Pellegrino, C. Ménager, F. Gazeau and C. Wilhelm, Biomaterials, 2014, 35, 6400-6411.

[39] COST, European Cooperation in Science and Technology, Brussels, 2014.

[40] M. A. Calin, G. Mologhianu, R. Savastru, M. R. Calin and C. M. Brailescu, Infrared Physics \& Technology, 2015, 69, 19-25.

[41] H. Wei, W. Yang, Q. Xi and X. Chen, Materials Letters, 2012, 82, 224-226.

[42] D. Bento, in Modelação matemática da variação da temperatura no pé, Master thesis, Polytechnic Institute of Bragança, Bragança, 2011. 
[43] Q. Liu, V. Barrón, J. Torrent, H. Qin and Y. Yu, Physics of the Earth and Planetary Interiors, 2010, 183, 387-397. 


\section{CHAPTER IV \\ Graphene-based magnetic nanoparticles for the treatment of cancer}

4.1 Introduction

4.2 Development of a tailor-made protocol to synthesize graphene-based yolk-shell magnetic nanoparticles for nanomedicine

4.3 Multifunctional graphene-based magnetic nanocarriers for combined hyperthermia and dual stimuli-responsive drug delivery 



\section{Graphene-based magnetic nanoparticles for the treatment of cancer}

\subsection{Introduction}

The frontiers of cancer research are currently focused on the design of multifunctional magnetic nanoparticles capable to achieve the synergistic cancer treatment, by combining the heat effect induced by hyperthermia and their unique drug delivery properties [1-3]. For this purpose, many magnetic nanoparticle-based drug delivery systems have been developed in the last decade [4, 5]. However, the major bottleneck for their combined clinical achievement has been the low drug loading capacity and poor controlleddrug release of the developed magnetic nanoparticles [6], as well as the low heating efficiency of the composite nanosystems in magnetic hyperthermia.

In this Chapter, the synthesis and optimization of graphene-based yolk-shell magnetic nanoparticles for nanomedicine are described, as well as its efficiency as multifunctional nanosystems in nanomedicine.

For this purpose, Section 4.2 details the development of a tailor-made protocol to synthesize graphene-based yolk-shell magnetic nanoparticles (GYSMNPs) for nanomedicine. The developed protocol shows the capability to synthesize several GYSMNPs sets with very distinctive physicochemical and toxicological properties, by just adjusting the amount of carbon precursors. The resulting sets of GYSMNPs were fully characterized regarding their chemical, physical and magnetic properties, envisioning their screening as efficient nanocarriers for combined biomedical applications. Study published in the journal $C$ Journal of Carbon Research (https://doi.org/10.3390/c4040055).

In Section 4.3, the GbMNP set developed in Section 4.2 that has revealed better combined physicochemical, magnetic and biocompatible properties, was selected to be tested as dual exogenous ( $\mathrm{AC}$ field) / endogenous $(\mathrm{pH})$ stimuli-responsive actuators for targeted thermochemotherapy, combining magnetic hyperthermia and controlled drug release triggered by the abnormal tumour environment.

The presented strategy and findings can represent a new way to design and develop highly stable added-value graphene-based nanostructures for the combined treatment of cancer.

The compilation of this later study was published in the journal Material Science Engineering C (https://doi.org/10.1016/j.msec.2018.07.060). 


\subsection{Development of a tailor-made protocol to synthesize graphene- based yolk-shell magnetic nanoparticles for nanomedicine}

In order to overcome magnetic nanoparticle limitations, such as toxicity, selfaggregation and low functionalization, many composite magnetic systems have been developed in the last decade for nanomedicine [4, 5]. These nanostructures are usually designed with a magnetic core coated with a metal or a non-metal structure, such as gold or silica. In general, this core-shell approach allows, beside the protection of the magnetic core, the anchoring of various therapeutic drugs or biomolecules for targeted drug delivery, improved biocompatibility and prolonged blood circulation half-life [3, 7]. Nevertheless, these systems also have some drawbacks, such as low drug loading capacity and lack of stimuli-responsive controlled release, when developed for drug delivery applications. Furthermore, the laborious synthetic protocols associated to these systems hinders the technology transfer to industry. Thus, additional efforts are necessary to add capping scaffolds on the nanoparticles surface (e.g., inorganic nanoparticles, polymers and biomacromolecules) that will act as gatekeepers to perform the drug controlled release in response to endogenous (i.e. pH, enzymes, among others) or exogenous (i.e. light, temperature changes, among others) stimuli $[2,8]$.

Carbon-based magnetic nanoparticles offer high chemical and thermal stability, large surface area, biocompatibility and can be easily functionalized [8, 9]. Recent studies, such as those published by Huang et al., 2016 [2] and Sasikale et al., 2016 [1], have shown that graphene-based materials, including graphene derivatives such as graphene oxide (GO), can be used as $\mathrm{pH}$ stimuli-responsive controlled release systems. This promising capability of graphene-based materials is due to $\pi-\pi$ stacking interactions that allow the adsorption of a variety of aromatic biomolecules, and to the presence of oxygen and hydrogen-containing surface groups that promote hydrogen bond interactions. However, the synthesis of magnetic nanoparticles combined with graphene derivatives demands, in general, toxic chemicals (e.g., those employed in the Hummers' method to produce GO [10]), or complex techniques/methods (e.g., in chemical vapour deposition [11]), often producing small quantities of nanoparticles. Moreover, the majority of the magnetic drug nanocarriers reported in literature, revealed a very low saturation magnetization $\left(M_{s}\right)$ profile $[1,8,9,12$, 13], limiting the utility of these magnetic nanocomposites.

To overcome these limitations, a simple protocol to synthesize tailor-made hydrophilic graphene-based yolk-shell magnetic nanoparticles (GYSMNPs) is herein detailed by adjusting the amount of carbon precursors in the coating of superparamagnetic magnetite 
nanoparticles (developed and characterized in Section 3.2). These tailor-made GYSMNPs were further characterized regarding their physical, chemical and magnetic properties with the aim to be further applied in nanomedicine.

\subsubsection{Materials and methods}

\subsubsection{Chemicals}

Ammonium hydroxide solution, $\mathrm{NH}_{4} \mathrm{OH}$ ( 25 wt.\% in $\mathrm{H}_{2} \mathrm{O}$ ), was purchased from Merck (NJ, USA). Ethanol absolute, $\mathrm{C}_{2} \mathrm{H}_{6} \mathrm{O}$ (99.8\%), nitric acid, $\mathrm{HNO}_{3}$ (70 wt.\%) and sodium hydroxide, $\mathrm{NaOH}$ (98 wt.\%), were obtained from Sigma-Aldrich (MO, USA). Formaldehyde (37 wt.\% stabilized with methanol) was supplied by Panreac (Barcelona, Spain). Resorcinol (98 wt.\%) was supplied by Fisher chemicals (MA, USA). Tetraethyl orthosilicate, TEOS (98 wt.\%), was obtained from Fluka (MO, USA). RPMI 1640 medium, hank's balanced salt solution (HBSS), fetal bovine serum (FBS), L-glutamine, trypsin-EDTA and penicillin/streptomycin solution were purchased from Gibco Invitrogen Life Technologies (Carlsbad, CA, USA). All aqueous solutions were prepared using ultrapure water $(18.2 \mathrm{M} \Omega . \mathrm{cm}$ at $25{ }^{\circ} \mathrm{C}$ ), produced in a Milli-Q system (Millipore), or deionized water.

\subsubsection{Synthesis of GYSMNPs}

The synthesis procedure of GYSMNPs was divided in two main steps: (i) synthesis of the superparamagnetic core, and (ii) formation of the graphene-based yolk-shell architecture.

In the first step, the superparamagnetic magnetite $\left(\mathrm{Fe}_{3} \mathrm{O}_{4}\right)$ core, with mean diameter of $18 \mathrm{~nm}$, was synthesized through co-precipitation of $\mathrm{Fe}^{2+}$ and $\mathrm{Fe}^{3+}$ (with a molar ratio of 1:2) in a basic solution of ammonium hydroxide, at $55{ }^{\circ} \mathrm{C}$, adapting the procedure described elsewhere [14], and as detailed in Section 3.2.1.2.

Afterwards, in the second step, the graphene-based yolk-shell architecture was achieved via one-pot strategy of hydrolysis and polymerisation of the precursors resorcinol, formaldehyde and TEOS. Different GYSMNPs, with different hollow cavities between the shell and magnetic core, were obtained using different amounts of the precursors resorcinol, formaldehyde and TEOS, in relation to a fixed mass of the magnetic core, adapting the procedure described elsewhere [15] (c.f. Table 4.1). In brief, an ultra-sonicated mixture of $0.25 \mathrm{~g}$ of magnetite with $150 \mathrm{~mL}$ of ethanol and $50 \mathrm{~mL}$ of deionized water was transferred into a $250 \mathrm{~mL}$ two- 
necked round-bottom flask. Resorcinol and ammonium hydroxide were further added to the mixture and magnetically stirred for $1 \mathrm{~h}$ at $30^{\circ} \mathrm{C}$. After that, the formaldehyde solution and TEOS were added dropwise and maintained under stirring at $30^{\circ} \mathrm{C}$ for $6 \mathrm{~h}$. The mixture was then heated at $80^{\circ} \mathrm{C}$ for another $8 \mathrm{~h}$ under constant stirring. The resulting magnetic product was washed by centrifugation several times with deionized water and absolute ethanol, and dried overnight at $60{ }^{\circ} \mathrm{C}$. The obtained samples were annealed under a $\mathrm{N}_{2}$ flow $\left(100 \mathrm{~cm}^{3} \mathrm{~min}\right.$ ${ }^{1}$ ) in a tubular vertical oven at 120 and $400{ }^{\circ} \mathrm{C}$ during $60 \mathrm{~min}$ at each temperature, and then at $600{ }^{\circ} \mathrm{C}$ for $240 \mathrm{~min}$, defining a heating ramp of $2^{\circ} \mathrm{C} \mathrm{min}{ }^{-1}$. Lastly, silica (positioned between the magnetic core and the outer shell) was removed by an etching process in a strong basic solution of sodium hydroxide (10 $\mathrm{mol} \mathrm{L}^{-1}, 16 \mathrm{~h}$ at room temperature with stirring), resulting in different sets of GYSMNPs.

\subsubsection{Hydrophilization of GYSMNPs}

To ensure the colloidal stabilization of the GYSMNPs in aqueous solutions, the graphene-based shells were chemically functionalized with the incorporation of carboxylic acid groups. For this purpose, an acid treatment at mild conditions was performed without compromising the magnetic core. In brief, the functionalization run was conducted in a $250 \mathrm{~mL}$ round-bottom flask at $65^{\circ} \mathrm{C}$ under vigorous magnetic stirring (C-Mag HS7, IKA). The samples of GYSMNPs $\left(5 \mathrm{mg} \mathrm{mL}{ }^{-1}\right)$ were oxidized with $\mathrm{HNO}_{3}\left(1 \mathrm{~mol} \mathrm{~L}{ }^{-1}\right)$ during $6 \mathrm{~h}$. The activated GYSMNPs were washed several times in deionized water to remove impurities. The last washing step was carried out with absolute ethanol, and the resulting material was dried overnight at $60^{\circ} \mathrm{C}$.

\subsubsection{Characterization of GYSMNPs}

The phase formation and crystallographic state of the materials were investigated in an Expert Pro Philips X-Ray diffractometer. Wide angle powder X-ray diffraction (XRD) analysis was carried out using a CuKa radiation $\left(\lambda=1.54^{\circ}\right)$.

The Raman spectra of the samples were recorded at ambient temperature with a 20x objective, using a micro-Raman spectrometer apparatus (Micro-Raman DXR, Thermo scientific) with a $532 \mathrm{~nm}$ laser excitation. Curve fitting for the Raman data was carried with Origin 8.0 software using the Lorentz function.

The morphology and microstructure of the GYSMNPs was evaluated using a probe Cs corrected High-Resolution Scanning Transmission Electron Microscope (HR-STEM), model Jeol ARM 200 CF. 
The amounts of carbon and magnetic core of the as-synthesized GYSMNPs were determined by thermogravimetric (TG) analysis (STA 449 C Jupiter, Netzsch), heating the samples in air flow from 50 to $950{ }^{\circ} \mathrm{C}$, at $20^{\circ} \mathrm{C} \mathrm{min}^{-1}$.

The $\mathrm{pH}$ at the point of zero charge $\left(\mathrm{pH}_{\mathrm{Pzc}}\right)$ of the materials was determined adapting the methodology described elsewhere [16]. Briefly, solutions with varying initial pH (2 - 12) were prepared using $\mathrm{HCl}\left(1.0 \mathrm{~mol} \mathrm{~L}^{-1}\right)$ or $\mathrm{NaOH}\left(1.0 \mathrm{~mol} \mathrm{~L}^{-1}\right)$ and $20 \mathrm{~mL}$ of $\mathrm{NaCl}\left(0.01 \mathrm{~mol} \mathrm{~L}^{-1}\right)$ as electrolyte. Each solution was contacted with $0.05 \mathrm{~g}$ of the material and the final $\mathrm{pH}$ was measured after $48 \mathrm{~h}$ of continuous stirring at room temperature. The $\mathrm{pH}_{\mathrm{PzC}}$ was determined by intercepting the obtained final $\mathrm{pH}$ vs. initial $\mathrm{pH}$ curve with the straight line final $\mathrm{pH}=$ initial $\mathrm{pH}$.

The magnetic properties of the superparamagnetic core $\left(\mathrm{Fe}_{3} \mathrm{O}_{4}\right)$ and GYSMNPs were investigated with a superconducting quantum interference device (SQUID-VSM) magnetometer from Quantum Design. Hysteresis curves were recorded for magnetic fields between $-20 \mathrm{kOe}$ and $20 \mathrm{kOe}$ at $27^{\circ} \mathrm{C}$.

\subsubsection{In vitro biostudies}

(i) Cell culture

Porcine liver primary cells (PLP2), established in a previous study [17], were used for the biocompatibility assays. Prior to the in vitro studies, cell lines were cultured in RPMI1640 medium containing $10 \%$ foetal bovine serum (FBS), $1 \%$ of glutaraldehyde, $1 \%$ of penicillin and $1 \%$ of streptomycin, under a humid atmosphere containing $5 \%$ of $\mathrm{CO}_{2}$, at $37^{\circ} \mathrm{C}$. The culture medium was replaced every two days, after the evaluation of the normal cell growth and morphology.

\section{(ii) Biocompatibility assay}

In vitro biocompatibility was assessed by using sulforhodamine B (SRB) colorimetric assay, as described elsewhere [18]. Briefly, the cell lines were seeded in 96-well plates, at an initial cell density of $1.0 \times 10^{4}$ cells/well. The biocompatibility of the GYSMNPs was assessed with the primary cell culture, PLP2, using different concentrations of the developed GYSMNPs $\left(0.12\right.$ to $\left.30.0 \mu \mathrm{g} \mathrm{mL}^{-1}\right)$. All the assays were performed in two individual tissue-culture plates and in duplicate wells $(n=4)$. Statistical analysis were performed using Microsoft Office Excel (version Professional Plus 2013). 


\subsubsection{Results and discussion}

\subsubsection{Synthesis and characterization of GYSMNPs}

Figure 4.1 illustrates the protocol that was followed for the synthesis of the superparamagnetic core and the formation of the graphene-based yolk-shell architecture.

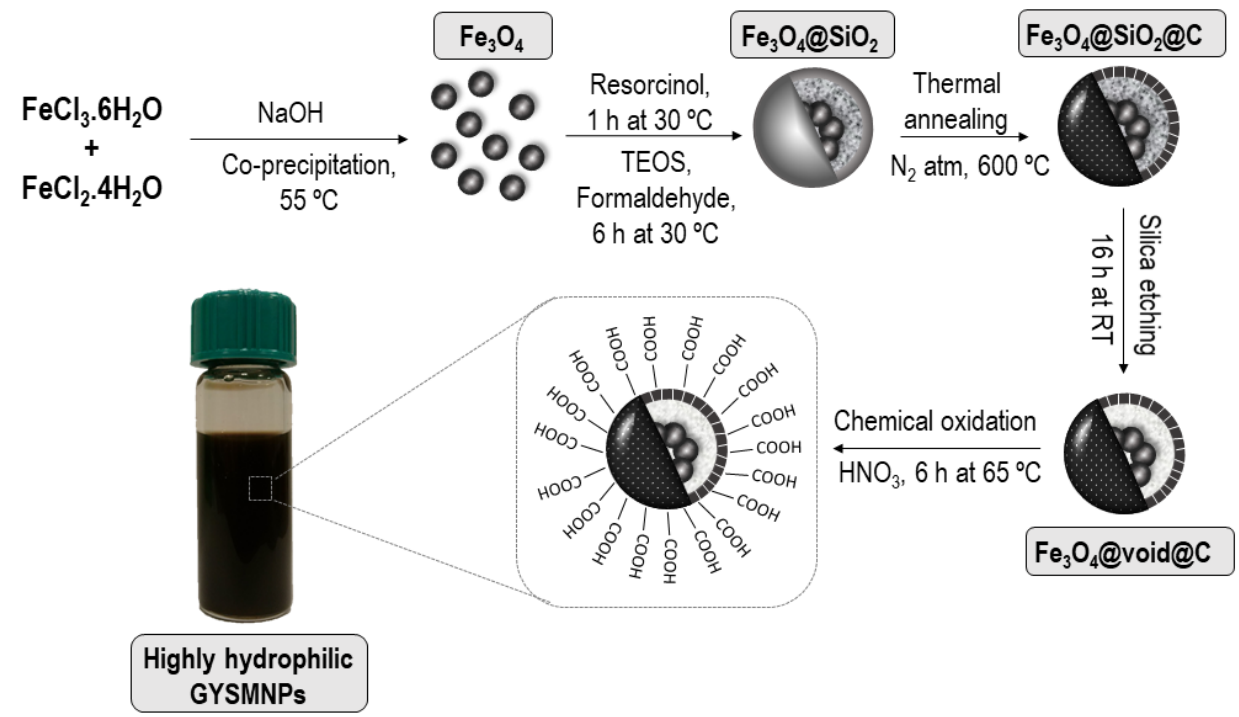

Figure 4.1. Schematic representation of the synthesis steps involved in the development of the hydrophilic graphene-based yolk-shell magnetic nanoparticles (GYSMNPs).

HR-STEM images (Figure 4.2) confirm the formation of yolk-shell magnetic nanoparticles, namely a small cluster of magnetite cores covered by a graphene-based layer. As expected, the increment of the amount of yolk-shell precursors (resorcinol, formaldehyde and TEOS), in relation to a fixed mass of the magnetic core, allowed the increase of the void and graphene-based shell thickness, as determined using ImageJ software (Table 4.1). Thus, three different types of GYSMNPs were synthesized with different hollow cavities and graphene-based shell thicknesses. 

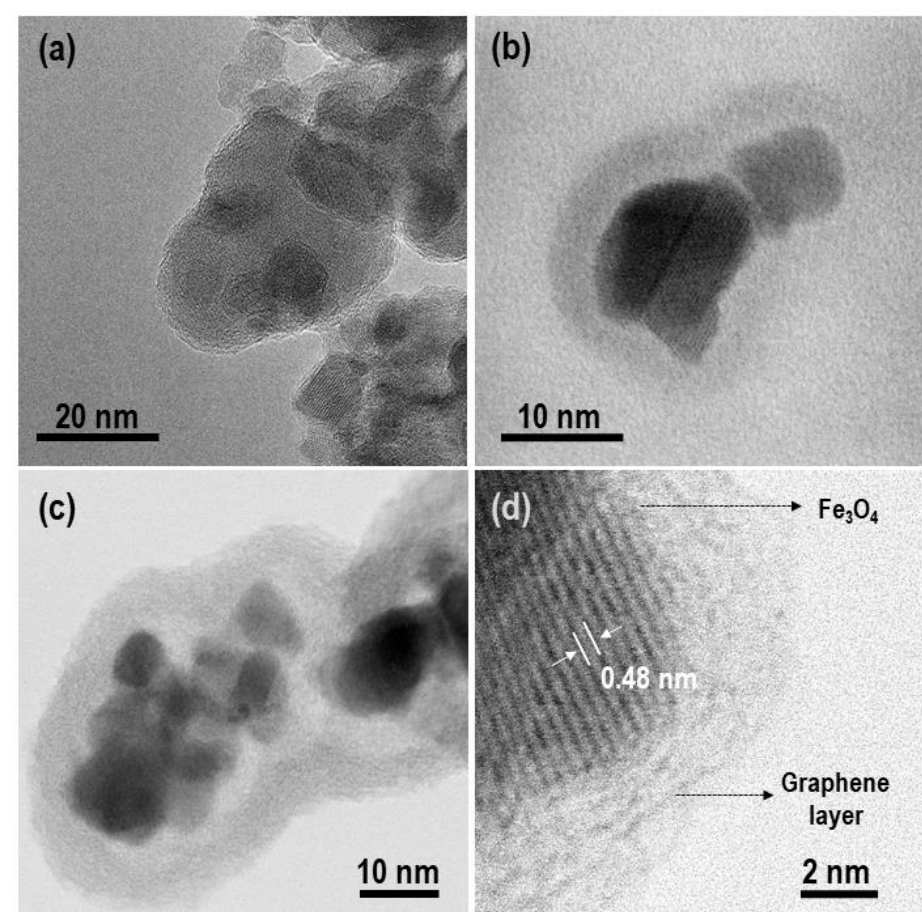

Figure 4.2. High resolution scanning transmission electron microscopy (HR-STEM) bright field images of: (a) sample GYSMNPs-1; (b) sample GYSMNPs-2; (c) sample GYSMNPs-3; (d) detail of the magnetic core of magnetite covered by few graphene layers of sample GYSMNPs- 2 .

Interestingly, the graphene-based shell thickness increased in the same proportion as the amount of precursors used. Thus, by doubling the carbon precursors from GYSMNPs-1 to GYSMNPs-2 and GYSMNPs-3, the shell thickness was successively doubled, as shown in Table 4.1.

Table 4.1. Effect of the amount of the precursor resorcinol, formaldehyde and TEOS, over the shell and void thickness of GYSMNPs

\begin{tabular}{ccccccc}
\hline Material & $\begin{array}{c}\text { Magnetic core } \\
(\mathbf{g})\end{array}$ & $\begin{array}{c}\text { Resorcinol } \\
(\mathbf{g})\end{array}$ & $\begin{array}{c}\text { Formaldehyde } \\
(\mathbf{m L})\end{array}$ & $\begin{array}{c}\text { TEOS } \\
(\mathrm{mL})\end{array}$ & $\begin{array}{c}\text { Void thickness } \\
(\mathbf{n m})^{\mathrm{a}}\end{array}$ & $\begin{array}{c}\text { Shell thickness } \\
(\mathrm{nm})^{\mathrm{a}}\end{array}$ \\
\hline GYSMNPs-1 & 0.25 & 0.05 & 0.075 & 0.10 & Not detected & $1.41 \pm 0.44$ \\
GYSMNPs-2 & 0.25 & 0.10 & 0.150 & 0.21 & $0.70 \pm 0.30$ & $3.55 \pm 1.27$ \\
GYSMNPs-3 & 0.25 & 0.20 & 0.300 & 0.41 & $2.07 \pm 0.92$ & $7.07 \pm 1.88$ \\
\hline
\end{tabular}

a Estimated by using ImageJ software from HR-STEM images as mean \pm standard deviation $(\mathrm{n}=60)$

HR-STEM images also allowed the determination of the lattice spacing of $0.48 \mathrm{~nm}$, suggesting that the magnetic core consists of $\mathrm{Fe}_{3} \mathrm{O}_{4}$ (Figure 4.2 (d)), as further confirmed by XRD analysis (Figure 4.3(a)). 

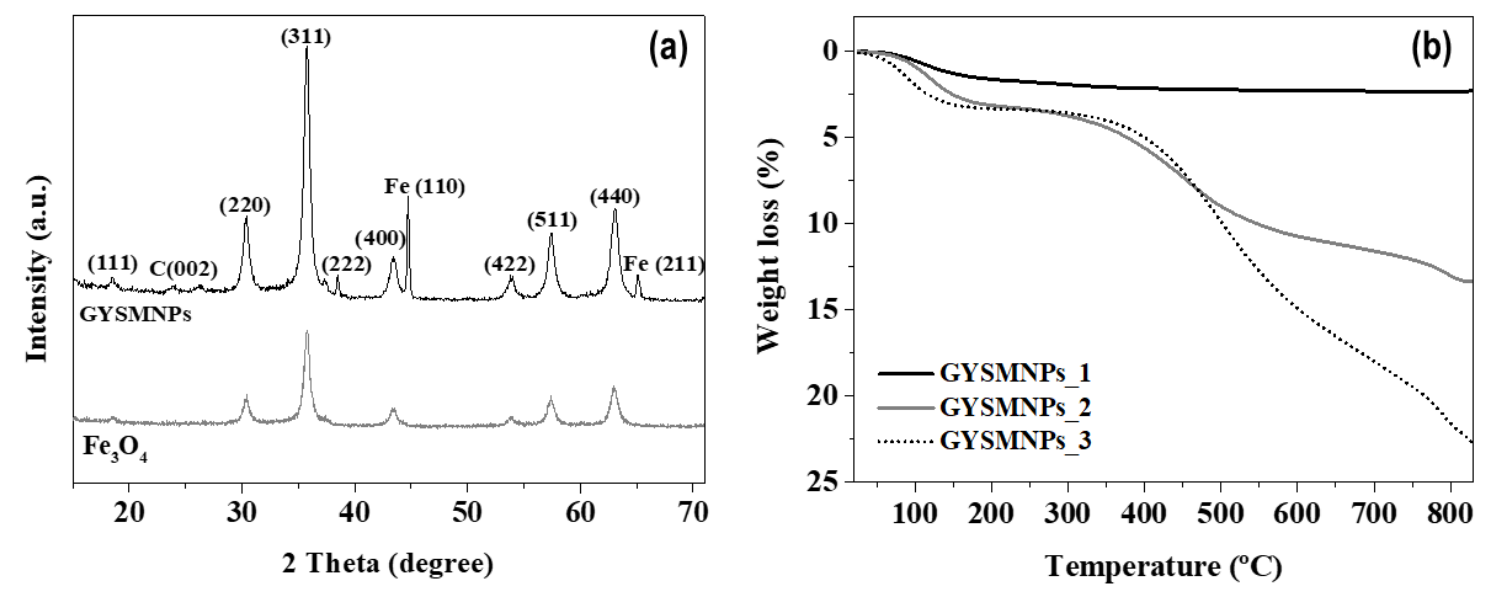

Figure 4.3. (a) Wide angle powder X-ray diffraction (XRD) patterns of $\mathrm{Fe}_{3} \mathrm{O}_{4}$ nanoparticles and GYSMNPs-2; (b) Thermogravimetric analysis of the as-synthesized graphene-based yolk-shell magnetic nanoparticles (GYSMNPs).

Results from thermogravimetric (TG) analysis are shown in Figure 4.3(b). The first significant weight loss below $150{ }^{\circ} \mathrm{C}$ can be attributed to the desorption of water molecules at the surface of the GYSMNPs samples. Above $300{ }^{\circ} \mathrm{C}$, the weight loss can be ascribed to the desorption of stable oxygen functional groups in the graphene-based shells, at such temperatures typically assigned to acidic carboxylic groups [19]. The sharp mass loss observed around 450 to $600{ }^{\circ} \mathrm{C}$, corresponds to the combustion of the carbon material, suggesting a mass percentage of the graphene-based shells in the samples of $0.5 \%, 9.7 \%$ and 18.2\% for GYSMNPs-1, GYSMNPs-2 and GYSMNPs-3, respectively. The mass percentage of magnetic core was calculated to be $97.7 \%, 88.0 \%$ and $77.8 \%$, respectively for GYSMNPs-1, GYSMNPs-2 and GYSMNPs-3.

Figures 4.4(a) and (b) show the Raman spectra of GYSMNPs-2 and GYSMNPs-3, respectively.
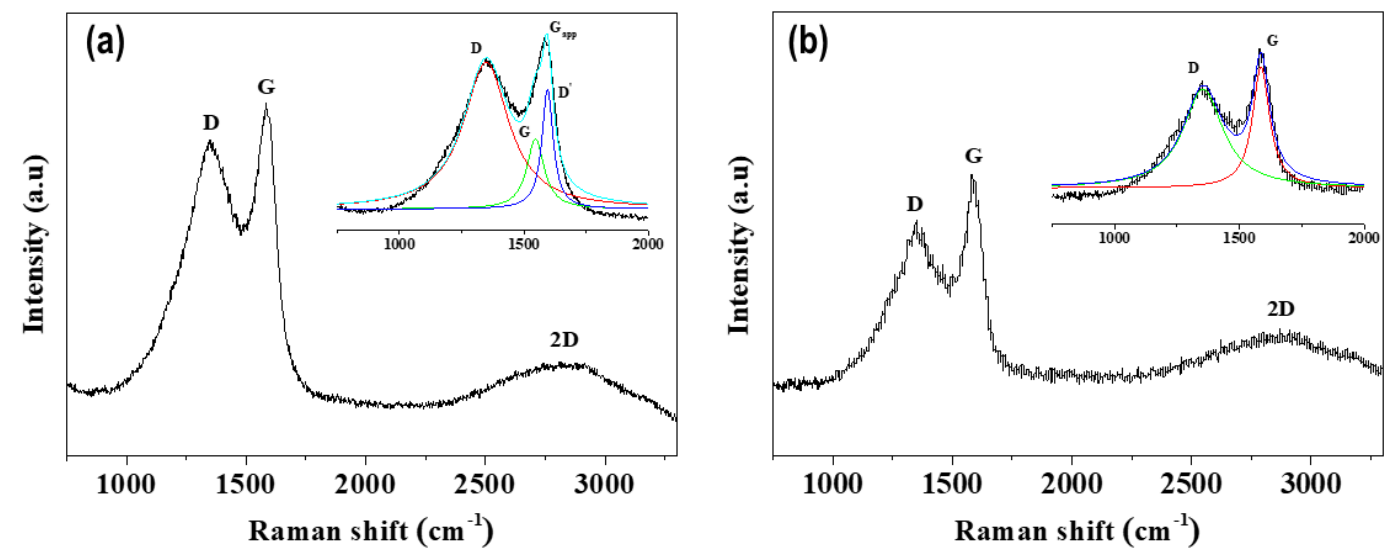

Figure 4.4. Raman spectra of as-synthesized GYSMNPs: (a) sample GYSMNPs-2 with inset corresponding to the deconvolution of the observed $D$ and apparent $G\left(G_{\text {app }}\right)$ bands into actual $G$ and D; (b) sample GYSMNPs-3 with inset corresponding to the deconvolution of the observed $D$ and $G$ bands. 
The presence of graphene oxide (GO) is suggested by the $D$ peak at $1350 \mathrm{~cm}^{-1}$, the conventional $G$ peak at $1582 \mathrm{~cm}^{-1}$ and the $2 D$ peak at $2816 \mathrm{~cm}^{-1}$ [20]. On the other hand, the Raman spectrum of sample GYSMNPs-1 did not show any of these peaks (data not shown), possible due to the very low amount of graphene-based shell $(0.5 \%$, determined by TG analysis). For this reason, sample GYSMNPs-1 was not considered in the following studies.

To confirm the type of graphene-based material, the three peaks ( $D, G$ and 2D) were analysed regarding their intensities, shapes, widths, positions and inter-correlations [21]. The $G$ band corresponds to an $E_{2 g}$ mode of hexagonal graphite and the vibration of $\mathrm{sp}^{2}$-hybridized carbon domain, while the $\mathrm{D}$ and $2 \mathrm{D}$ peaks are assigned to structural defects $[8,9]$. The integrated peak intensity between $I_{D}$ and $I_{G}$ was found to be 1.61 for sample GYSMNPs-2, corresponding to a disordered GO structure [20, 22], and 0.43 for sample GYSMNPs-3, indicating the presence of a defective multi-graphitic-layer shell [23] further supported by HR-STEM observations (Figure 4.2(c)). Moreover, by the deconvolution of the conventional $G$ band - or the apparent $G$ band $\left(G_{\text {app }}\right)$, actual $G\left(1546 \mathrm{~cm}^{-1}\right)$ and $D^{\prime}\left(1594 \mathrm{~cm}^{-1}\right)$ bands can be assigned in sample GYSMNPs-2, but not clearly in sample GYSMNPs-3. The presence of the D' band was ascribed by Kaniyoor and Ramaprabhu [24] as proof of the presence of a highly defective structure comprising few layers of wrinkled graphene-based structures, which is similar to the shell nanostructure found in GYSMNPs-2 (cf. Figure 4.2 (d)).

The main textural properties of the synthesized GYSMNPs are given in Table 4.2. The specific surface area $\left(S_{\mathrm{BET}}\right)$ of GYSMNPs-2 and GYSMNPs-3 materials was found to be 156 and $245 \mathrm{~m}^{2} \mathrm{~g}^{-1}$, respectively. These relatively high values obtained in comparison with $56 \mathrm{~m}^{2} \mathrm{~g}^{-1}$ for uncoated magnetite (data not shown), are ascribed to the presence of the graphenebased shell in the GYSMNPs structure. Thus, it seems that the $S_{\mathrm{BET}}$ increased with the shell thickness. The analysis of the $V_{\text {micro }} / V_{\text {total }}$ ratio $(0.041$ and 0.105 for GYSMNPs- 2 and GYSMNPs-3, respectively), reveals that the samples mainly contain mesoporous. In addition, a decrease of the average pore diameter was observed from GYSMNPs-2 to GYSMNPs-3 (8.2 to $5.4 \mathrm{~nm}$, respectively). Overall, GYSMNPs samples, in comparison with uncoated magnetite, exhibit large surface areas and mesoporous nature, which is very advantageous for the encapsulation, transport and release of guest molecules such as chemotherapeutic drugs for drug delivery applications. 
Table 4.2. Textural properties of the synthesized materials: specific surface area $\left(\mathrm{S}_{\mathrm{BET}}\right)$, non-microporous surface area $\left(S_{\text {meso }}\right)$, micropore volume $\left(V_{\text {micro }}\right)$, total pore volume $\left(V_{\text {total }}\right)$, ratio between micropore and total pore volume $\left(V_{\text {micro }} / V_{\text {total }}\right)$ and average pore diameter $\left(d_{\text {average }}\right)$

\begin{tabular}{ccccccc}
\hline Material & $\begin{array}{c}S_{\text {BET }} \\
\left(\mathrm{m}^{2} \mathbf{g}^{-1}\right)\end{array}$ & $\begin{array}{c}S_{\text {meso }} \\
\left(\mathrm{m}^{2} \mathbf{g}^{-1}\right)\end{array}$ & $\begin{array}{c}V_{\text {micro }} \\
\left(\mathrm{cm}^{3} \mathbf{g}^{-1}\right)\end{array}$ & $\begin{array}{c}V_{\text {total }} \\
\left(\mathrm{cm}^{3} \mathbf{g}^{-1}\right)\end{array}$ & $V_{\text {micro }} / V_{\text {total }}$ & $\begin{array}{c}d_{\text {average }} \\
(\mathrm{nm})\end{array}$ \\
\hline GYSMNPs-2 & 156 & 123 & 0.013 & 0.318 & 0.041 & 8.2 \\
GYSMNPs-3 & 245 & 160 & 0.035 & 0.333 & 0.105 & 5.4 \\
\hline
\end{tabular}

Figure 4.5 shows the magnetic hysteresis curves obtained for $\mathrm{Fe}_{3} \mathrm{O}_{4}$ and GYSMNPs. The determined magnetic properties of these samples are listed in Table 4.3. $\mathrm{Fe}_{3} \mathrm{O}_{4}$ exhibits a saturation magnetization $\left(M_{s}\right)$ of 77.7 emu $\mathrm{g}^{-1}$ (Fe304), but, as expected, the magnetic susceptibilities of the GYSMNPs suffer a decrease with the increase of the graphene-based shell thickness, 69.8 and 61.2 emu $^{-1}{ }_{(\mathrm{Fe} 304)}$, respectively for GYSMNPs-2 and GYSMNPs-3. However, and impressively, the $M_{\mathrm{s}}$ for these magnetic nanoparticles are much higher than determined in other yolk-shell nanoparticles reported in literature $[1,8,9,15]$, possible due to the presence of superparamagnetic magnetite clusters as magnetic core, as suggested by HR-STEM images. More interesting, the superparamagnetic-like behaviour of the GYSMNPs was maintained, as revealed by the low-field data (Table 4.3 and inset in Figure 4.5), with negligible saturation remanence $\left(M_{r}\right)$ and low coercivity $\left(H_{c}\right)$.

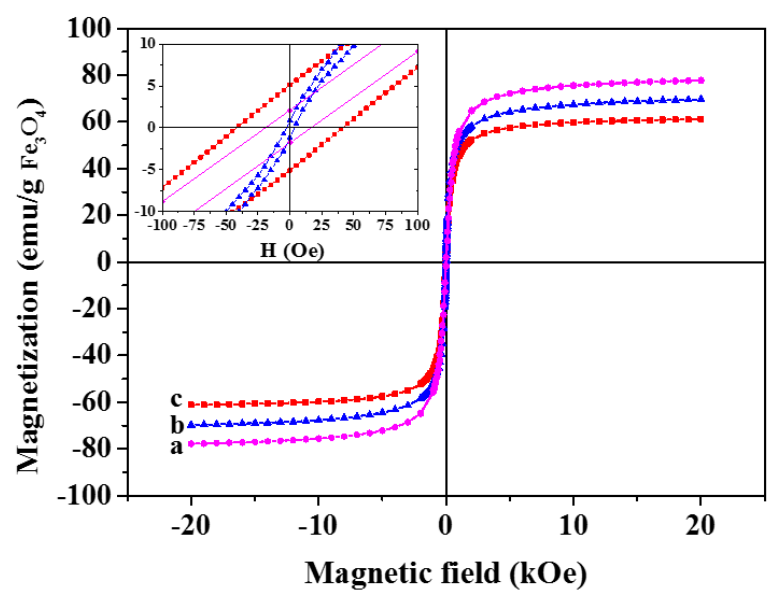

Figure 4.5. Magnetization curves of (a) $\mathrm{Fe}_{3} \mathrm{O}_{4}$, (b) GYSMNPs-2 and (c) GYSMNPs-3. Hysteresis loops at $27^{\circ} \mathrm{C}$ up to $\pm 20 \mathrm{kOe}$; the inset is a zoom in the low field region.

Table 4.3. Magnetic properties of the uncoated $\mathrm{Fe}_{3} \mathrm{O}_{4}$ and as-synthesized GYSMNPs: saturation magnetization $\left(M_{\mathrm{s}}\right)$, coercivity $\left(H_{\mathrm{c}}\right)$ and saturation remanence $\left(M_{\mathrm{r}}\right)$

\begin{tabular}{cccc}
\hline Sample & $\begin{array}{c}M_{\mathrm{s}} \\
\left(\mathrm{emu} \mathrm{g}^{-1} \text { magnetite }\right)\end{array}$ & $\begin{array}{c}H_{\mathrm{c}} \\
(\mathrm{Oe})\end{array}$ & $\begin{array}{c}M_{\mathrm{r}} \\
\left(\mathrm{emu} \mathrm{g}^{-1} \text { magnetite) }\right.\end{array}$ \\
\hline $\mathrm{Fe}_{3} \mathrm{O}_{4}$ & 77.7 & 18.33 & 1.94 \\
$\mathrm{GYSMNPs}^{2}$ & 69.8 & 3.54 & 1.16 \\
GYSMNPs-3 & 61.2 & 41.33 & 5.08 \\
\hline
\end{tabular}




\subsubsection{In vitro biocompatibility assays}

Biocompatibility of nanomaterials is a key parameter for its potential application in biological systems, where an ideal nanocarrier should ensure low or negligible toxicity. GYSMNPs biocompatibility at different concentrations (from 0.12 to $30.0 \mu \mathrm{gL}^{-1}$ ) was determined in PLP2 cells and normalized to the cell viability in cell culture for $48 \mathrm{~h}$, at $37^{\circ} \mathrm{C}$ (Figure 4.6).

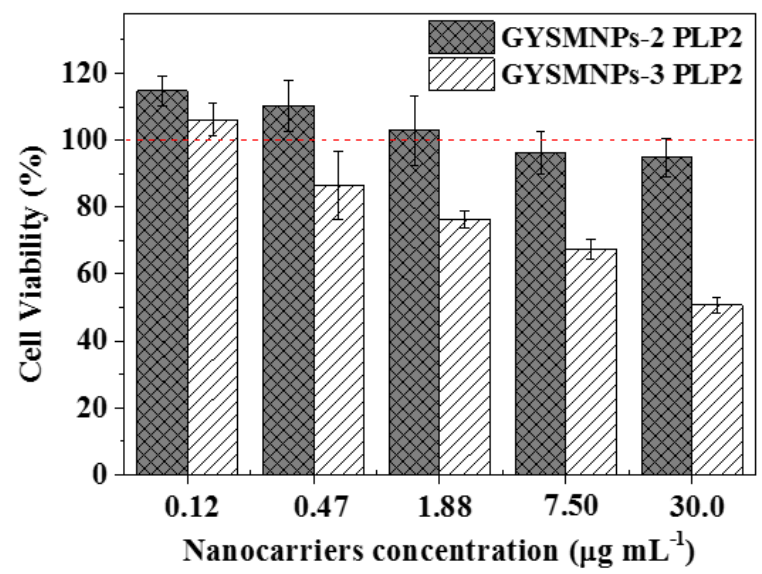

Figure 4.6. In vitro biocompatibility study of GYSMNPs in primary cell culture cells (PLP2). Error bars represent a $95 \%$ confidence interval.

The biocompatibility assays reveal a very distinct behaviour between the developed nanosystems, GYSMNPs-2 and GYSMNPs-3, tested in PLP2 cells. GYSMNPs-2, characterized by few layers GO yolk-shell magnetic nanoparticles (cf. Figure 4.4(a)), exhibits good biocompatibility even at the highest concentration of $30.0 \mu \mathrm{g} \mathrm{mL}^{-1}$. On the other hand, GYSMNPs-3, characterized as a yolk-shell magnetic nanostructure coated with a defective multi-graphitic-layer shell (cf. Figure 4.4(b)), show a severe cell inhibition behaviour at the highest concentrations tested. Indeed, these results are in line with others reported in the literature, where the cytotoxicity of different graphene-based nanomaterials has been discussed $[6,25,26]$. According to the available information, the lateral size, shape, number of layers, stiffness, hydrophobicity, surface functionalization, concentration dose and chemistry of the graphene-based materials, all play an important role in the interaction of this type of materials with cell membranes, intracellular uptake and its cytotoxicity [26]. For instance, good biocompatibility results, similar to those obtained with GYSMNPs-2, were obtained by Sasidharan and co-workers [27], after the functionalization of GO nanostructures with carboxyl groups that supressed its cytotoxicity by weakening hydrophobic interactions between cell membranes and GO. This ability to functionalize carbon-based nanomaterials can then be used to improve the biocompatibility of these nanosystems for biomedical applications. 
Overall, among the three different GYSMNPs that were developed on this Section 4.2, GYSMNPs-2 exhibits better combined features to be applied in nanomedicine (i.e., magnetic hyperthermia, drug delivery and gene delivery, among others).

\subsubsection{Conclusions}

In this section, hydrophilic graphene-based yolk-shell magnetic nanoparticles were developed envisioning biomedical applications, such as magnetic hyperthermia and/or drug delivery applications, by using a simple tailor-made coating protocol. The results show the capability to synthesize GYSMNPs with very distinctive physicochemical and toxicological properties, just by adjusting the amount of carbon precursors. Among the three distinct materials developed in this study, a hybrid magnetic nanomaterial labelled as GYSMNP-2, has shown exceptional characteristics to be applied in nanomedicine. Impressively, this hybrid nanomaterial exhibited: (1) high surface area; (2) exceptional magnetic saturation value of 69.8 emu $\mathrm{g}^{-1}$; (3) superparamagnetic behaviour; and (4) biocompatibility. The combination of these features suggests a high potentiality to apply these magnetic nanocomposites as combined nanosystems for the treatment of cancer, such as by combining magnetic hyperthermia with drug delivery applications, as investigated in the next section. 


\subsection{Multifunctional graphene-based magnetic nanocarriers for combined hyperthermia and dual stimuli-responsive drug delivery}

Graphene-based magnetic nanoparticles, due to their unique properties, such as high chemical and thermal stability, high charge carrier mobility and large surface area for functionalization [6,9], have the potentiality to be designed as efficient multifunctional nanocarrier systems with enhanced capability to protect the magnetic core from oxidation or dissolution in acidic environment $[1,28]$. Moreover, its unique $s p^{2}$ carbon structure with $\pi-\pi$ stacking and negative surface charge, enables the adsorption of a variety of molecules, e.g., chemotherapeutic drugs, DNA and/or RNA, which can be specifically accumulated into tumours, either by active targeting or by the enhanced permeability and retention effect $[1,8]$. In addition, this non-covalent interaction mechanism allows the use of graphenebased yolk-shell magnetic nanoparticles as $\mathrm{pH}$ stimuli-responsive controlled drug release systems, triggered by the abnormal acidic pH values (around 4.5 - 5.5) that are found in tumour endosome/lysosome microenvironments [29]. On the other hand, the superparamagnetic core of these nanocomposites allows heat generation in the tumour under an AC magnetic field that induces cancer cells death, by the so-called magnetic hyperthermia, and can simultaneously boost the drug release on those abnormal tissues. In fact, the combination of magnetic hyperthermia and chemotherapy has been demonstrated in clinical trials to have a synergistic effect on the cancer therapy response compared to the therapeutic effect achieved when these treatment procedures are applied alone [30]. In this regard, graphene-based magnetic nanomaterials, developed as multifunctional nanocarriers, are able to have a dramatic impact in medicine and in the treatment of cancer. However, the majority of the magnetic drug nanocarriers reported in the literature revealed a low saturation magnetization $\left(M_{s}\right)$ profile $[1,8,9,12,13]$, limiting the utility of these magnetic nanocomposites for magnetic hyperthermia. Also, the possible aggregation and precipitation of graphene-based materials in the presence of electrolyte solutions, such as the body fluids [31], is an issue that needs to be addressed.

With the aim to overcome these limitations, the sample graphene-based yolk-shell magnetic nanoparticles (GYSMNP-2) developed in Section 4.2, was selected to be optimized and tested as dual-nanosystem for combined hyperthermia and controlled-drug delivery. In order to improve its biocompatibility and colloidal stabilization, the GYSMNP material was functionalized with the copolymer pluronic F-127 (GYSMNP@PF127). Pluronic copolymers have been investigated for the development of thermo-responsive hydrogels and drug delivery systems [32]. Particularly, pluronic F-127, with the non-proprietary name poloxamer 407, is a water-soluble and biocompatible triblock copolymer - containing 
hydrophilic poly(ethylene oxide) (PEO) blocks and hydrophobic poly(ethylene oxide) (PPO) blocks arranged as PEO-PPO-PEO, and it is approved by the U.S. Food and Drug Administration (FDA) for use as food additive and in pharmaceutical formulations [33]. The grafting of nanoparticles with pluronic F-127 allows the incorporation of a hydrophilic PEO corona that reduces the aggregation of nanoparticles and the adsorption of blood proteins, as well as enhance the biocompatibility of the nanosystems and its colloidal stability, prolonging blood circulation [32, 34]. Additionally, the terminal hydroxyl functional groups allow the conjugation with selected ligands for the development of multifunctional nanosystems with high potential for active tumour targeting and therapeutic efficiency enhancement [34]. In this section, GYSMNP@PF127 was conjugated with doxorubicin (DOX), a highly potent chemotherapeutic drug clinically used against a broad spectrum of cancers. The developed therapeutic nanosystem was thoroughly characterized and investigated as multifunctional nanocarrier to combine magnetic mild hyperthermia and stimuli-responsive drug release for thermo-chemotherapy.

\subsubsection{Materials and methods}

\subsubsection{Chemicals}

Nitric acid, $\mathrm{HNO}_{3}$ (70 wt.\%), copolymer pluronic F-127, $\mathrm{PEO}_{106} \mathrm{PPO}_{70} \mathrm{PEO}_{106}$ $\left(M_{w}=12600\right)$, sodium chloride, $\mathrm{NaCl}(99.5 \mathrm{wt} . \%)$, sodium phosphate monobasic, $\mathrm{NaH}_{2} \mathrm{PO}_{4}$ (99.0 wt.\%), potassium chloride, $\mathrm{KCl}(99 \mathrm{wt} . \%)$, sodium phosphate dibasic, $\mathrm{Na}_{2} \mathrm{HPO}_{4}$ (99.0 wt.\%) and sodium hydroxide, $\mathrm{NaOH}$ (98 wt.\%), were obtained from Sigma-Aldrich (MO, USA). Doxorubicin hydrochloride, DOX (98 wt.\%), was purchased from Discovery Fine Chemicals (Wimborne, UK). Physiological salt solution (PSS) with $0.9 \% \mathrm{NaCl}$ was supplied by B. Braun Medical (Germany). Hoechst dye (BisBenzimide) and monoclonal anti-a-tubulin antibody produced in mouse (B512) was obtained from Sigma-Aldrich (Sant Louis, MO, USA). Goat anti-Mouse IgG $(\mathrm{H}+\mathrm{L})$ Highly Cross-Adsorbed Secondary Antibody, Alexa Fluor 488 was obtained from Invitrogen (USA). The cell line HepG2 was purchased from Deutshe Sammlung von Mikroorganismen and Zellkulturen Gmbit (DSMZ). RPMI 1640 medium, hank's balanced salt solution (HBSS), fetal bovine serum (FBS), L-glutamine, trypsin-EDTA, penicillin/streptomycin solution were purchased from Gibco Invitrogen Life Technologies (Carlsbad, CA, USA). All chemicals were used as received, without further purification. All aqueous solutions were prepared using ultrapure water $\left(18.2 \mathrm{M} \Omega . \mathrm{cm}\right.$ at $\left.25^{\circ} \mathrm{C}\right)$, produced in a Milli-Q system (Millipore), or deionized water. 


\subsubsection{Synthesis and colloidal optimization of GYSMNPs}

GYSMNP material was synthesized in two main steps: (i) synthesis of the superparamagnetic core, and (ii) formation of the graphene-based shell architecture, as previously described in Section 4.2.1.2.

The colloidal stabilization of GYSMNP (previously labelled in Section 4.2 as GYSMNPs-2) was performed by chemical functionalization of the graphene-based shell. For this purpose, an acid treatment at mild conditions was performed to incorporate carboxylic and hydroxyl groups, without compromising the magnetic core, and followed by grafting the copolymer pluronic F-127 (PF127) onto the surface of GYSMNP. PF127 was used as enhancer for both colloidal stabilization and biocompatibility of GYSMNP [35]. Briefly, the initial surface functionalization of GYSMNP was conducted in a $250 \mathrm{~mL}$ round-bottom flask at $65{ }^{\circ} \mathrm{C}$ under vigorous magnetic stirring (C-Mag HS7, IKA). The as-synthesized GYSMNP material (5 mg mL${ }^{-1}$ ) was oxidized with $\mathrm{HNO}_{3}\left(1 \mathrm{~mol} \mathrm{~L}^{-1}\right)$ during $3 \mathrm{~h}$, washed and dried overnight at $60{ }^{\circ} \mathrm{C}$, as previously reported in section 4.2.1.3. Once the surface was activated, GYSMNP $\left(2 \mathrm{mg} \mathrm{mL}^{-1}\right)$ was functionalized with PF127 $\left(40 \mathrm{mg} \mathrm{mL}^{-1}\right)$ for $5 \mathrm{~h}$ in magnetic stirring, at room temperature. The resulting solution was washed by centrifugation (13 $000 \mathrm{rpm}, 20 \mathrm{~min}$ ) with deionized water to remove unbounded copolymer, and the final GYSMNP@PF127 pellet redispersed in a phosphate buffer solution or deionized water at desired concentration. The obtained hydrophilic GYSMNP@PF127 material was further used to encapsulate the chemotherapeutic drug doxorubicin (DOX).

\subsubsection{Characterization of GYSMNPs}

The average hydrodynamic diameter and surface charge of GYSMNP@PF127 were determined by dynamic light scattering (DLS) and zeta potential (ZP) measurements (Zetasizer Nano, Malvern Instruments) at a concentration of $0.1 \mathrm{mg} \mathrm{mL}^{-1}$ dispersed in Milli-Q water at room temperature $(n=3)$.

The organic content of functionalized GYSMNP@PF127 was determined employing TG analysis under $\mathrm{N}_{2}$ atmosphere by heating the samples from 50 to $550{ }^{\circ} \mathrm{C}$ at a rate of $10{ }^{\circ} \mathrm{C} \mathrm{min}-1$, and comparing the results with those obtained with the as-synthesized GYSMNP, analysed under the same conditions and used as reference.

The $\mathrm{pH}$ at the point of zero charge $\left(\mathrm{pH}_{\mathrm{PzC}}\right)$ of the nanomaterials was determined adapting the methodology described elsewhere [16]. Briefly, solutions with varying initial pH (2 - 12) were prepared using $\mathrm{HCl}\left(1.0 \mathrm{~mol} \mathrm{~L}^{-1}\right)$ or $\mathrm{NaOH}\left(1.0 \mathrm{~mol} \mathrm{~L}^{-1}\right)$ and $20 \mathrm{~mL}$ of $\mathrm{NaCl}\left(0.01 \mathrm{~mol} \mathrm{~L}^{-1}\right)$ 
as electrolyte. Each solution was incubated with $0.05 \mathrm{~g}$ of the material and the final $\mathrm{pH}$ was measured after $48 \mathrm{~h}$ of continuous stirring at room temperature. The $\mathrm{pH}_{\mathrm{PzC}}$ was determined by intercepting the obtained final $\mathrm{pH}$ vs. initial $\mathrm{pH}$ curve with the straight line final $\mathrm{pH}=$ initial $\mathrm{pH}$.

Fourier transform infrared (FTIR) spectroscopy was performed with a Perkin Elmer Spectrum equipment using $\mathrm{KBr}$ pellets.

\subsubsection{Drug loading studies}

The drug loading experiments were carried out by mixing a given amount of DOX (from 50 to $500 \mu \mathrm{g} \mathrm{mL}^{-1}$ ) and a fixed amount of GYSMNP@PF127 $\left(500 \mu \mathrm{g} \mathrm{mL}^{-1}\right)$ in a phosphate buffer solution with $\mathrm{pH}$ 7.4. The suspensions were kept in a shaking incubator at room temperature during $48 \mathrm{~h}$ in darkness, and the loading tests were performed in triplicate. At the end of each experiment, the GYSMNP@PF127 material loaded with the drug (referred as GYSMNP@PF127-DOX) was collected by centrifugation at $15000 \mathrm{rpm}$ for $30 \mathrm{~min}$. The supernatant (containing the free drug) was used to monitor the absorbance at $480 \mathrm{~nm}$ (characteristic of DOX), and to determine the drug concentration. The drug loading efficiency and drug loading capacity were calculated using Equations (4.1) and (4.2), respectively.

Drug loading efficiency $(\%)=\frac{\text { Inicial concentration of drug-Drug content in the supernatant }}{\text { Inicial concentration of drug }} \times 100$

Drug loading capacity $\left(\mu \mathrm{g} \mathrm{mg}^{-1}\right)=\frac{\text { mass of drug loaded }}{\text { mass of GYSMNP@PF127 }}$

\subsubsection{5 $\mathrm{pH}$ and temperature-dependent drug release}

The $\mathrm{pH}$ and temperature dependent drug release of DOX loaded on GYSMNP@PF127 were evaluated in the absence of AMF (AC magnetic field). For this purpose, freeze-dried GYSMNP@PF127-DOX samples $(2.0 \mathrm{mg})$ suspended in phosphate buffer solution $(20 \mathrm{~mL})$ at physiological $(\mathrm{pH} \mathrm{7.4)}$ and acidic $\mathrm{pH}$ levels $(\mathrm{pH} 6.0$ and 4.5), were incubated at $37^{\circ} \mathrm{C}$ (physiological body temperature) and $45^{\circ} \mathrm{C}$ (hyperthermia temperature). At selected time intervals $(0.5,1,2,3,4,6,8,24$ and $48 \mathrm{~h})$, samples were collected, centrifuged, and the supernatant used to monitor the absorbance at $480 \mathrm{~nm}$ to determine the $\mathrm{pH}$ and temperature-dependent release of DOX. 


\subsubsection{DOX release kinetics analysis}

First-order (release dependent of concentration), Higuchi (release by diffusion) and Korsmeyer-Peppas (log (cumulative drug release) vs log (time)) models were fitted to drug release data in order to understand the drug release kinetics. The kinetic model that best fitted the drug release data was selected by comparing the respective regression coefficients $\left(R^{2}\right)$ of the different models. The Korsmeyer-Peppas model is used to characterize different release mechanisms from polymeric systems by using the $n$ (release exponent) value. For cylindrical shaped matrices, values of $n<0.45$ means quasi-Fickian diffusion, $n=0.45$ means Fickian diffusion, $0.45<n<0.89$ means anomalous diffusion (diffusion + erosion), $n=0.89$ means non-Fickian case II (zero order) and $n>0.89$ means non-Fickian supercase II [36].

\subsubsection{Validation of GYSMNP@PF127-DOX as dual $\mathrm{pH}$ - and AMF/temperature- responsive drug nanocarrier}

GYSMNP@PF127 heating efficiencies were first evaluated in a hyperthermia equipment (Fives Celes, France) under an oscillating magnetic field of $H=21.0 \mathrm{kA} \mathrm{m}^{-1}$ and $340 \mathrm{kHz}$. For this purpose, $0.3 \mathrm{~mL}$ of GYSMNP@PF127 was dispersed in water at a concentration of $1.5 \mathrm{mg} \mathrm{mL}^{-1}$ and placed in the mid-point of a cupper coil. Then, the temperature increase was logged as a function of time during the external AMF application using an optical fibre probe centred in the nanoparticle dispersion. The heating measurements started at stabilized room temperature and were limited to $10 \mathrm{~min}$. The specific absorption rate (SAR, W g $\left.(\mathrm{Fe})^{-1}\right)$ was calculated according to Equation (2.5). In addition, the intrinsic power loss (IPL, $\mathrm{nHm}^{2} \mathrm{Kg}^{-1}$ ), which is independent of the magnetic field parameters and allows direct comparison between different experiments, was calculated using Equation (3.3).

On another set of experiments, the dual $\mathrm{pH}$ and $\mathrm{AMF} /$ temperature-responsive drug release of GYSMNP@PF127-DOX were determined under the same AMF conditions $\left(H=21.0 \mathrm{kA} \mathrm{m}^{-1}\right.$ and $f=340 \mathrm{kHz})$ at a concentration of $3.0 \mathrm{mg} \mathrm{mL}^{-1}$. For this purpose, freeze-dried GYSMNP@PF127-DOX nanocarrier was dispersed in $0.3 \mathrm{~mL}$ of phosphate buffer at $\mathrm{pH} 7.4$ or 4.5, and maintained under AMF for $30 \mathrm{~min}$. After that, the supernatant was centrifuged and used to monitor the absorbance at $480 \mathrm{~nm}$ to determine the $\mathrm{pH}$ - and AMF/temperaturedependent release of DOX under magnetic hyperthermia. 


\subsubsection{In vitro cell tests}

\section{(i) Haemocompatibility studies}

The blood-nanocarriers biocompatibility was tested by haemolysis assay according to previous reports [14, 37]. Briefly, human whole blood from healthy donors was collected into $2.7 \mathrm{~mL}$ tubes (S-Monovette, Sarstedt) containing ethylenediaminetetraacetic acid (EDTA). The whole blood was diluted in a ratio 1:2 (v/v) in PSS (physiological salt solution) and centrifuged twice at $2500 \mathrm{rpm}$ for $10 \mathrm{~min}$ to isolate the red blood cells (RBCs). The isolated RBCs were diluted in $15 \mathrm{~mL}$ of PSS $0.4 \mathrm{~mL}$ of diluted RBCs suspension was exposed to $1.6 \mathrm{~mL}$ of GYSMNP@PF127 at different concentrations $(10,50,100,200,400$, $500 \mu \mathrm{g} \mathrm{mL}^{-1}$ ). Positive control was obtained using the $0.4 \mathrm{~mL}$ of RBCs diluted $1.6 \mathrm{~mL}$ of PSS buffer and the negative control by adding to the diluted whole blood, deionized water in the same volume of $(1: 1, v / v)$. Samples were incubated on an orbital shaker at $37^{\circ} \mathrm{C}$ during $3 \mathrm{~h}$. The released haemoglobin was determined after centrifugation at $2500 \mathrm{rpm}$ for $10 \mathrm{~min}$, followed by photometric analysis of the supernatant at $570 \mathrm{~nm}$. The haemolysis ratio (HR) in percentage was calculated from the optical (OD) density using Equation (3.1) [14], from two independent experiments in triplicates (mean \pm standard deviation).

(ii) Cell culture

Two cell lines were used for the cytotoxicity assays, namely porcine liver primary cells (PLP2), established by some of the authors [17], and a human liver cancer cell line (HepG2). HepG2 was used for cellular uptake of drug nanocarriers. Prior to the in vitro cell studies, both cell lines were cultured in RPMI-1640 medium containing 10\% foetal bovine serum (FBS), $1 \%$ of glutaraldehyde, $1 \%$ of penicillin and $1 \%$ of streptomycin, under a humid atmosphere containing $5 \%$ of $\mathrm{CO}_{2}$, at $37{ }^{\circ} \mathrm{C}$. The culture medium was replaced every two days, after the evaluation of the normal cell growth and morphology.

(iii) Cell cytotoxicity assays and cellular uptake of drug nanocarriers

The cytotoxicity of GYSMNP@PF127 and GYSMNP@PF127-DOX was assessed in PLP2 and HepG2, respectively, using the sulfurhodamine B (SRB) colorimetric assay as described elsewhere [18]. SRB assay has been widely used for drug-toxicity testing in different types of cancerous and non-cancerous cell lines [38]. Practical advances makes the SRB assay very sensitive to measure drug-induced cytotoxicity and cell proliferation even at large-scale drug-screening applications [39]. Briefly, $100 \mu \mathrm{L}$ of RPMI-1640 complete medium containing different concentrations of GYSMNP@PF127 or GYSMNP@PF127-DOX, ranging from 
0.05 to $50 \mu \mathrm{g} \mathrm{mL}^{-1}$, were seeded in 96 -well plates. Immediately after, $100 \mu \mathrm{L}$ of RPMI-1640 complete medium containing the respective cell lines in test were added to each well, at an initial cell density of $1.0 \times 10^{4}$. In addition, control wells containing just cells with cultured medium were seeded and used as blank and control. All the assays were performed in two individual tissue-culture plates in duplicated wells $(\mathrm{n}=4)$ and cultured in $5 \% \mathrm{CO}_{2}$, at $37{ }^{\circ} \mathrm{C}$ for $48 \mathrm{~h}$. The percent cell viability was calculated considering the untreated cells as $100 \%$ viability. The results were used to determine the GI50, as the concentration that inhibits the growth of cells by $50 \%$.

Moreover, immunostaining experiments to observe drug internalization on cells were performed with cancer cell line. HepG2 cell line was incubated with $5 \mu \mathrm{g} \mathrm{mL}^{-1}$ of GYSMNP@PF127-DOX during 2, 4 and 6 h. Cells were fixed in 4\% paraformaldehyde. Hoechst dye was used to stain DNA. Microtubules were immunolabeled with the monoclonal antitubulin antibody (B512) that was recognized by a secondary goat anti-mouse immunoglobulin G (IgG) conjugated Alexa Fluor 488. Confocal microscopy images were obtained with a Carl Zeiss inverted microscope attached to the LSM 780 confocal system (software; ZEN 2010).

\subsubsection{Results and discussion}

\subsubsection{Synthesis and characterization of GYSMNP@PF127}

Figure 4.7 illustrates the whole protocol of synthesis that was implemented in this study (including the steps represented in Figure 4.1).

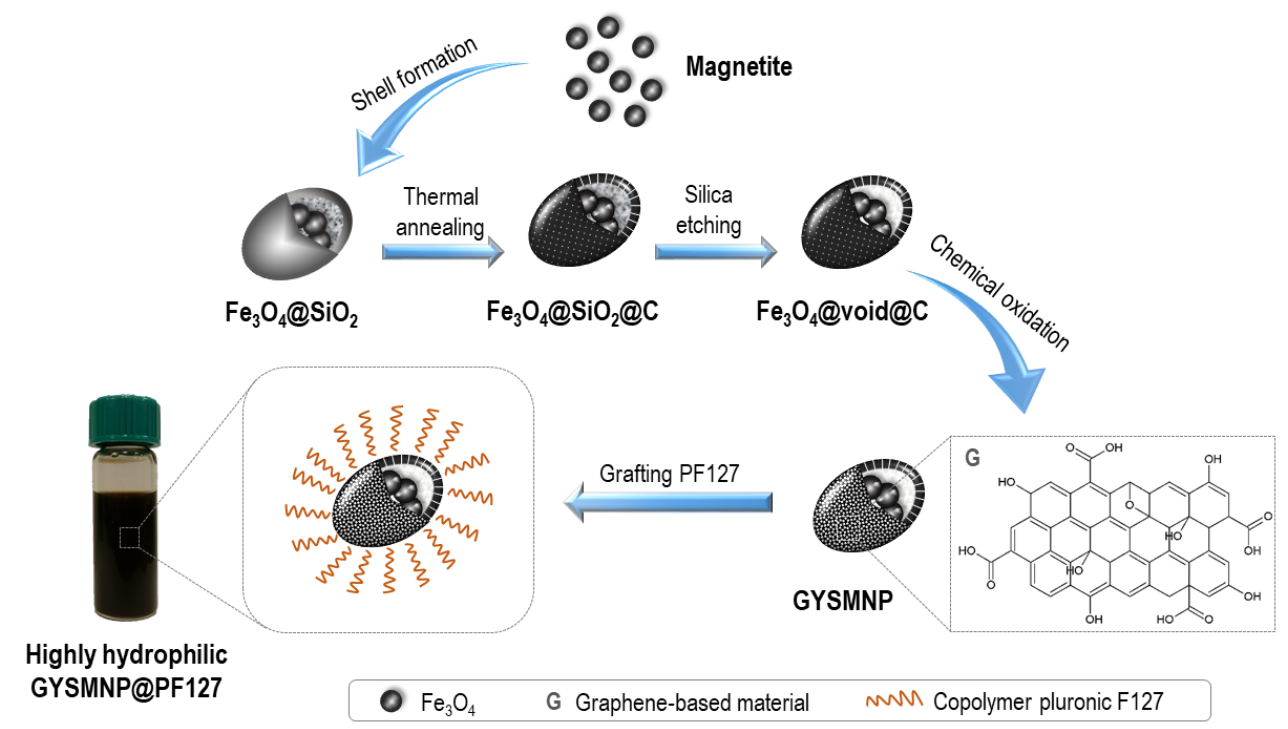

Figure 4.7. Schematic overview of the steps involved in the development and grafting of the hydrophilic graphene-based yolk-shell magnetic nanoparticles (GYSMNP@PF127). 
The GYSMNP material with the best physicochemical, magnetic and biocompatible properties (previously labelled in Section 4.2 as GYSMNP-2), was functionalized with PF127, envisioning the optimization of the colloidal and biocompatible features.

It is important to note that for nanomedicine, the proposed nanosystem has to show excellent colloidal stabilization in the presence of electrolyte solutions, optimal average hydrodynamic dimension $\left(D_{\mathrm{H}}\right)$, low haemotoxicity and good biocompatibility. In this context, the GYSMNP@PF127 nanosystem was further characterized taking into account those properties. $D_{H}$ of GYSMNP@PF127 was found to be $180 \mathrm{~nm}$ (Figure 4.8), with polydispersion index (PDI) of 0.14 (Table 4.4). Due to the enhanced permeability and retention (EPR) effect, nanoparticles with size range of $10-200 \mathrm{~nm}$ are ascribed to be preferentially accumulated into tumour rather than in healthy tissues [30]. Particles in the range of 100-200 $\mathrm{nm}$ are large enough to avoid uptake by the liver and splenic filtration [40]. Thus, the $D_{H}$ of GYSMNP@PF127 shows an optimal dimension for suitable intravenous administration and EPR effect with prolonged blood circulation.

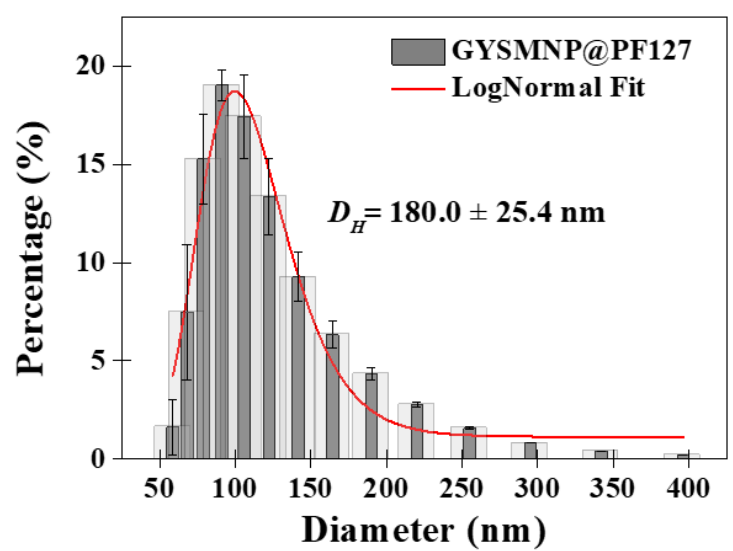

Figure 4.8. DLS measurements with average hydrodynamic diameter $\left(D_{H}\right)$.

Additionally, the zeta potential of GYSMNP, before and after functionalization with nitric acid and pluronic F-127 (PF127), was determined. A higher negative value, around - $37 \mathrm{mV}$, was determined for the functionalized samples with respect to as-synthesized GYSMNP, with zeta potential around - $17 \mathrm{mV}$ (data not shown).

These results indicate the incorporation of carboxylic acid groups and negative charged PF127 on the surface of GYSMNP, with a consequent increase in the colloidal stability. Moreover, negative surface charge is ascribed to increased nanoparticle circulation by avoiding opsonisation phenomenon and their elimination from blood by macrophage cells [41]. 
Table 4.4. Colloidal and dimensional properties of GYSMNP@PF127: void thickness, hydrodynamic diameter $\left(D_{\mathrm{H}}\right)$, polydispersion (PDI) and zeta potential.

\begin{tabular}{cccccc}
\hline Material & $\begin{array}{c}\text { Void thickness } \\
(\mathrm{nm})^{\mathrm{a}}\end{array}$ & $\begin{array}{c}\text { Shell thickness } \\
(\mathrm{nm})^{\mathrm{a}}\end{array}$ & $\begin{array}{c}D_{H} \\
(\mathrm{~nm})^{\mathrm{b}}\end{array}$ & PDI $^{\mathrm{b}}$ & $\begin{array}{c}\text { Zeta Potential } \\
(\mathrm{mV})^{\mathrm{b}}\end{array}$ \\
\hline GYSMNP@PF127 & $0.7 \pm 0.3$ & $3.6 \pm 1.3$ & $180.0 \pm 25.4$ & $0.14 \pm 0.02$ & $-36.8 \pm 2.0$ \\
\hline
\end{tabular}

a Estimated by using ImageJ software from HR-STEM images as mean \pm standard deviation $(\mathrm{n}=60)$

${ }^{b}$ Estimated by DLS and zeta potential measurements, in deionized water at $0.1 \mathrm{mg} \mathrm{mL}^{-1}$, represented as mean \pm standard deviation $(n=3)$

Figure 4.9 (a) shows the Fourier transform infrared (FTIR) spectra of the samples collected at different stages of the preparation process. All samples have various oxygen-containing functional groups in their structure including hydroxyl, namely $\mathrm{C}-\mathrm{OH}\left(3050-3800 \mathrm{~cm}^{-1}\right.$ and $\left.1070 \mathrm{~cm}^{-1}\right)$, carboxyl $\left(\mathrm{COOH}, 1650-1750 \mathrm{~cm}^{-1}\right)$, ketonic $\left(\mathrm{C}=0,1600-1650 \mathrm{~cm}^{-1}\right.$ and 1750-1850 $\mathrm{cm}^{-1}$ ) and epoxide (C-O-C, $1230-1320 \mathrm{~cm}^{-1}$ ) species, as well as $\mathrm{sp}^{2}$-hybried $\mathrm{C}=\mathrm{C}$ $\left(1600-1680 \mathrm{~cm}^{-1}\right)$ and alkanes $\left(\mathrm{C}-\mathrm{H}, 1460 \mathrm{~cm}^{-1}\right.$ and $\left.1370-1385 \mathrm{~cm}^{-1}\right)$, which are in accordance with previous reports for graphene oxide (GO) samples [37]. Moreover, a peak at $570 \mathrm{~cm}^{-1}$, which is characteristic for the stretching vibration of Fe-O bond from magnetite [38], was observed in all samples. Particularly, an additional peak at $1106 \mathrm{~cm}^{-1}$ (C-0, 1085-1300 $\mathrm{cm}^{-1}$ ) was verified for GYSMNP@PF127 (sample (iii) in Figure 4.9 (a)), as a result of the incorporation of PF-127 onto its structure. FTIR characterization of GYSMNP@PF127 shows a variety of oxygen-containing functional groups that are favourable for the encapsulation, transport and release of guest molecules, such as chemotherapeutic drugs for drug delivery applications.
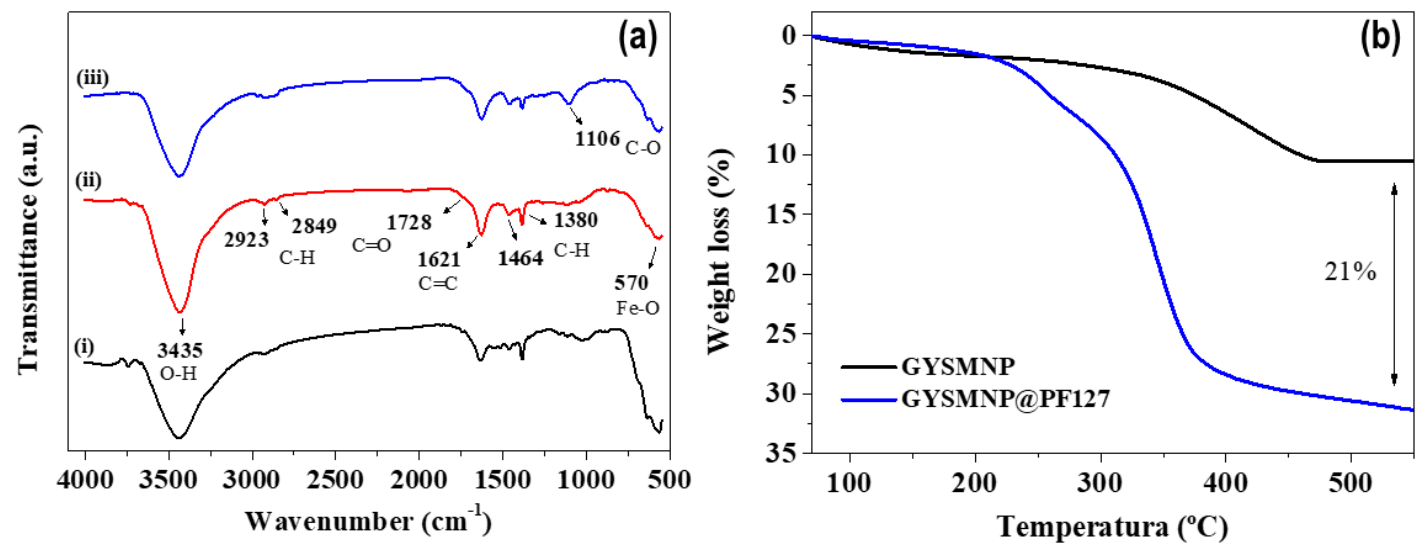

Figure 4.9. (a) FTIR spectra of (i) as-synthesized GYSMNP, (ii) GYSMNP after activation with nitric acid, (iii) GYSMNP after grafting with pluronic F-127 (GYSMNP@PF127); (b) Thermogravimetric analysis of the as-synthesized and functionalized GYSMNP materials under $\mathrm{N}_{2}$ atmosphere.

The organic content of the samples was determined by TG analysis under inert atmosphere, ranging the temperature from 50 to $550{ }^{\circ} \mathrm{C}$. Figure 4.9 (b) shows two sharp weight losses for GYSMNP@PF127. The first, occurring between 100 and $270{ }^{\circ} \mathrm{C}$, can be ascribed to the 
decomposition of same surface oxygen groups [39], whereas the second, between 300 and $500{ }^{\circ} \mathrm{C}$, corresponds to the thermal decomposition of PF-127 [40], with a weight loss of $21 \%$ in comparison to GYSMNP.

Thus, the successful incorporation of PF127 into the graphene-based shell of the magnetic nanosystem was achieved, which improved the structural and colloidal features of the assynthesized GYSMNP in order to be applied in nanomedicine.

\subsubsection{DOX loading studies}

Doxorubicin (DOX) is a cationic chemotherapeutic drug and one of the most commonly used against cancer [42]. However, it presents lethal side effects on healthy cells too [43]. Targeted drug delivery can be an effective method to overcome this issue by delivering the cargo specifically into tumours after being triggered by its abnormal acidic microenvironment. Thus, DOX was chosen to explore the drug delivery properties of GYSMNP@PF127, which shows promising potential to carry high payloads of cationic drugs due to its negative surface charge, optimal size range, large surface area, mesoporous nature and hollow cavity. The drug loading efficiency and drug loading capability were assessed by mixing DOX concentrations between 50 to $500 \mu \mathrm{g} \mathrm{mL}^{-1}$ with a fixed amount of nanoparticles $\left(500 \mu \mathrm{g} \mathrm{mL}^{-1}\right)$. The loading data are shown in Table 4.5.

Table 4.5. Drug loading on GYSMNP@PF127 at different initial DOX concentrations ( $50-500 \mu \mathrm{g} \mathrm{mL}^{-1}$ ) in phosphate buffer $\mathrm{pH}$ 7.4. Standard deviation of triplicate drug loading tests $(n=3)$.

\begin{tabular}{ccccccc}
\hline & \multicolumn{6}{c}{ Initial DOX concentration $\left(\mu \mathrm{g} \mathrm{mL}^{-1}\right)$} \\
& 50 & 100 & 200 & 300 & 400 & 500 \\
\hline $\begin{array}{c}\text { Drug capacity } \\
\left(\mu \mathrm{g} \mathrm{mg}^{-1}\right)\end{array}$ & $96.0 \pm 1.0$ & $196.5 \pm 0.4$ & $394.1 \pm 1.0$ & $587.0 \pm 0.5$ & $771.2 \pm 9.0$ & $910.5 \pm 11.0$ \\
\hline $\begin{array}{c}\text { Drug efficiency } \\
(\%)\end{array}$ & $96.0 \%$ & $98.3 \%$ & $98.5 \%$ & $97.8 \%$ & $96.4 \%$ & $91.1 \%$ \\
\hline
\end{tabular}

Impressively, the drug loading capacity of GYSMNP@PF127 increases with the initial DOX concentration, reaching an outstanding $910 \mu \mathrm{g}$ DOX mg-1 GYSMNP@PF127 when the initial DOX concentration is $500 \mu \mathrm{g} \mathrm{mL} \mathrm{mL}^{-1}$, which represents a $91 \%$ of drug loading efficiency $(1: 1, w / w)$. These drug loading results show the extraordinary capability of GYSMNP@PF127 to load high contents of DOX. This ability can be ascribed to the $\pi-\pi$ stacking interactions between the aromatic rings of DOX and the carbonaceous structure of graphene-based nanoshells [47], as well as to some surface groups. The post-synthetic functionalization of GYSMNP allowed a high negative surface charge (zeta potential values ca. - $37 \mathrm{mV}$ (Table 4.4)) with $\mathrm{pH}_{\mathrm{PZC}}$ around 2 (i.e., $\mathrm{pH}$ at which the surface charge is zero), which also 
allows electrostatic interactions with positively charged DOX $(\mathrm{pKa}=8.3)$ at $\mathrm{pH} 7.4$ [48]. Thus, besides the $\pi-\pi$ stacking between the aromatic rings of DOX molecules and the graphene-based nanoparticles, the high DOX loading on GYSMNP@PF127 is also a consequence of the electrostatic interactions and the mesoporous structure of the graphenebased shell.

\subsubsection{Dual $\mathrm{pH}$ - and temperature-dependent drug release}

It is well-known that tumour microenvironment presents a weak acidic $\mathrm{pH}$ value, around 6.0 - 7.2, at the tumour tissue due to the Warburg effect, which dramatically decrease to severe acidic $\mathrm{pH}$ values, around 4.5 - 5.5, in tumour endosome/lysosome environment [29]. The $\mathrm{pH}$ differences between the tumour microenvironment and normal tissues, with $\mathrm{pH}$ around 7.4, motivate the development of $\mathrm{pH}$-dependent/responsive nanocarriers that can be specifically designed as smart drug delivery systems triggered by acidic $\mathrm{pH}$ values. Therefore, the cumulative $\mathrm{pH}$-dependent DOX release of GYSMNP@PF127-DOX was studied under different $\mathrm{pH}$ conditions (7.4, 6.0 and 4.5, corresponding to the mimicked physiological $\mathrm{pH}$, tumour microenvironment and intracellular tumour endosome/lysosome $\mathrm{pH}$ conditions, respectively) either at $37{ }^{\circ} \mathrm{C}$ (physiological temperature) or $45^{\circ} \mathrm{C}$ (hyperthermia temperature), cf. Figure 4.10.

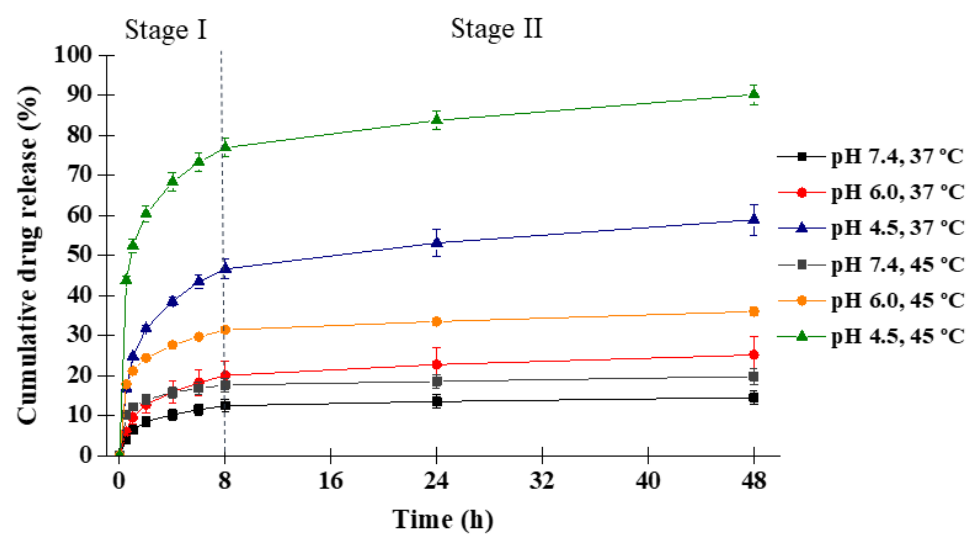

Figure 4.10. $\mathrm{pH}$ and thermo-responsive release of doxorubicin (DOX) from drug loaded GYSMNP@PF127 under different pH values (7.4, 6.0 and 4.5) and temperatures ( 37 and $45{ }^{\circ} \mathrm{C}$ ). Standard deviation of triplicate drug release tests $(n=3)$.

Figure 4.10 reveals that GYSMNP@PF127 has a pronounced $\mathrm{pH}$ - and temperature-dependency drug delivery behaviour. The drug release at these conditions takes place in two stages: (I) a rapid DOX release in the first $8 \mathrm{~h}$, followed by (II) a slower rate release till $48 \mathrm{~h}$.

Analysis of stage (I) shows that just a very low amount of DOX was released at physiological $\mathrm{pH}$ conditions (i.e. $\mathrm{pH} 7.4$ ), both at 37 and $45^{\circ} \mathrm{C}$. The drug release was slightly higher at $\mathrm{pH}$ 
6.0 than at $\mathrm{pH} 7.4$, i.e., $20 \%$ versus $12 \%$ at $37^{\circ} \mathrm{C}$, and $31 \%$ versus $18 \%$ at $45^{\circ} \mathrm{C}$. But remarkably, at $\mathrm{pH} 4.5$, the drug nanocarriers show an increase of the released DOX around $47 \%$ at $37{ }^{\circ} \mathrm{C}$, and an impressive $76 \%$ at $45^{\circ} \mathrm{C}$.

For the long-period drug release, stage (II), just a maximum of $20 \%$ of DOX was released under physiological conditions. Under $\mathrm{pH} 6.0$, a maximum drug release of $25 \%$ and $36 \%$ was achieved for 37 and $45{ }^{\circ} \mathrm{C}$, respectively, whereas at $\mathrm{pH} 4.5$ a remarkable $91 \%$ of the encapsulated drug was released at $45^{\circ} \mathrm{C}$.

Once again, the main reasons behind this promising $\mathrm{pH} /$ temperature-responsive drug release behaviour of the graphene-based material comes from the $\pi-\pi$ stacking between the graphene-based nanostructures and the aromatic DOX molecules, which can be easily disrupted under a mild acidic environment [8], but also due to the increased solubility of DOX caused by the protonation process [9]. In this particular example, this $\mathrm{pH}$-dependent controlled release phenomenon can be also ascribed to the increased protonation of the GYSMNP@PF127 nanocarrier surface as the $\mathrm{pH}$ of the aqueous solution decreases from 7.4 to 4.5 , with the associated loss of negative surface charge. This will lead to a reduction of the electrostatic attraction between GYSMNP@PF127 and DOX protonated molecules, increasing the progressive drug release.

Overall, the $\mathrm{pH}$-responsive drug release results obtained in this work suggest the great ability of the designed graphene-based magnetic nanocarriers to be applied as efficient drug encapsulation and delivery systems, with an especial good $\mathrm{pH}$ sensitivity for an intelligent and on-demand drug release on acidic tumour microenvironments.

\subsubsection{Drug release kinetics}

The results of the kinetic modelling, performed with the drug release data obtained for GYSMNP@PF127 (at 37 and $45{ }^{\circ} \mathrm{C}$ and different pHs), are listed in Table 4.6. In this study, three kinetic models, namely first-order, Higuchi and Korsmeyer-Peppas, were fitted to the drug release data obtained for each $\mathrm{pH}$ and temperature tested condition. The model with the higher $R^{2}$ values (for each condition) was considered as the best model. 
Table 4.6. Drug release kinetic analysis of GYSMNP@PF127-DOX.

\begin{tabular}{ccccccccc}
\hline \multirow{2}{*}{ Temperature } & pH & \multicolumn{2}{c}{ First-order } & \multicolumn{2}{c}{ Higuchi } & \multicolumn{3}{c}{ Korsmeyer-Peppas } \\
\cline { 2 - 8 } & & $K_{1}$ & $R^{2}$ & $H_{h}$ & $R^{2}$ & $K_{k p}$ & $n$ & $R^{2}$ \\
\hline \multirow{3}{*}{$37^{\circ} \mathrm{C}$} & 7.4 & 0.001 & 0.556 & 1.456 & 0.743 & 0.009 & 0.20 & 0.932 \\
& 6.0 & 0.002 & 0.655 & 2.781 & 0.816 & 0.013 & 0.23 & 0.948 \\
& 4.5 & 0.005 & 0.734 & 6.074 & 0.827 & 0.033 & 0.22 & 0.962 \\
\hline \multirow{3}{*}{$45^{\circ} \mathrm{C}$} & 7.4 & 0.001 & 0.575 & 1.346 & 0.758 & 0.016 & 0.13 & 0.977 \\
& 6.0 & 0.002 & 0.638 & 2.608 & 0.797 & 0.027 & 0.14 & 0.980 \\
& 4.5 & 0.014 & 0.849 & 6.676 & 0.818 & 0.066 & 0.14 & 0.984 \\
\hline
\end{tabular}

The first-order and Higuchi kinetic models have very low $R^{2}$ values (slightly higher for the Higuchi model). These results suggest that the drug release, under the tested conditions, is not mainly concentration-dependent or total controlled by diffusion behaviour. The kinetic model that fits better (higher $R^{2}$ ) is the Korsmeyer-Peppas model, as expected, since it considers two parameters instead of one. The $n$ values calculated from the KorsmeyerPeppas model equation were found to be lower than 0.45 , suggesting that the drug release is essentially controlled by simple quasi-Fickian diffusion mechanism.

\subsubsection{Validation of GYSMNP@PF127-DOX as dual $\mathrm{pH}$ - and AMF/temperature- responsive drug nanocarrier}

The observed $\mathrm{pH}$ - and temperature-dependence drug delivery profiles highlight the convenience of exploiting the magnetic properties of the magnetite cores to induce a temperature increase by external application of an AMF (AC magnetic field). Thus, in addition to an endogenous stimuli $(\mathrm{pH})$ that boosts the localized drug release on the tumour site, we can externally enhance this effect by inducing a temperature increase through magnetic hyperthermia.

The response of GYSMNP@PF127-DOX as dual pH- and temperature-sensitive drug release nanocarrier was assessed under AMF $\left(f=340 \mathrm{kHz}\right.$ and $\left.H=21.0 \mathrm{kA} \mathrm{m}^{-1}\right)$ during $30 \mathrm{~min}$. For this purpose, $3.0 \mathrm{mg} \mathrm{mL}^{-1}$ of GYSMNP@PF127-DOX, which corresponds to $1.6 \mathrm{mg} \mathrm{mL}^{-1}$ of unloadedDOX, GYSMNP@PF127, was dispersed in phosphate buffer at $\mathrm{pH} 7.4$ and 4.5. Mild hyperthermia temperature $\left(40-43^{\circ} \mathrm{C}\right)$ was reached between 10 and $13 \mathrm{~min}$ and maintained till the end of the hyperthermia experiment (30 min) (Figure 4.11). 
Multifunctional GbMNPs for combined hyperthermia and dual stimuli-responsive drug delivery
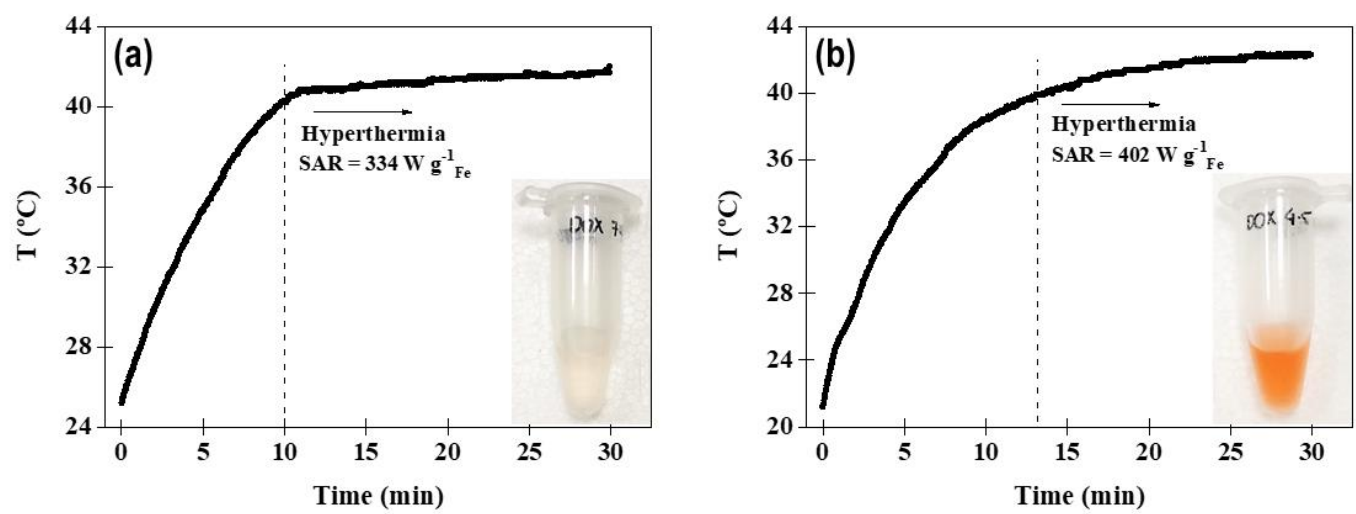

Figure 4.11. Magnetic-induced thermal response curve of GYSMNP@PF127-DOX (3.0 mg GYSMNP-DOX $\mathrm{mL}^{-1}$ ) for dual $\mathrm{pH}$ - and thermal-responsive drug delivery: (a) dispersed in phosphate buffer at 7.4 to mimic physiological microenvironment, and (b) dispersed in phosphate buffer at 4.5 to mimic tumour microenvironment. Samples were subjected to an AMF ( $f=340 \mathrm{kHz}$ and $\mathrm{H}=21.0 \mathrm{kA} \mathrm{m}^{-1}$ ) during 30 min. Inset shows the drug-containing supernatant resulting from each hyperthermia experiment.

From the initial slope of the heating curve, SAR (specific absorption rate) and IPL (intrinsic power loss) values were estimated to be 334 and $402 \mathrm{~W} \mathrm{~g} \mathrm{ge}^{-1}$, which correspond to 2.2 and $2.7 \mathrm{nHm}^{2} \mathrm{Kg}^{-1}$, from samples dispersed in phosphate buffer 7.4 and 4.5 , respectively. These results represent a much better heating efficiency than other magnetic nanomaterials synthesized for hyperthermia application [28, 30, 44], and are in agreement with IPL values $\left(0.15-3.1 \mathrm{nHm}^{2} \mathrm{Kg}^{-1}\right)$ of commercial ferrofluids [30,45]. Moreover, these SAR and IPL values are similar to those obtained for the unloaded-DOX GYSMNP@PF127 (Figure 4.12), showing that the loading of the drug does not significantly change the original heating performance.

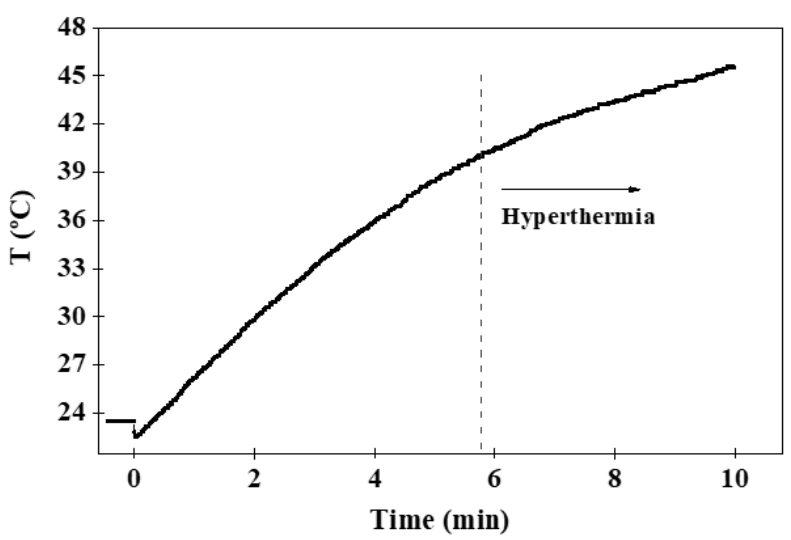

Figure 4.12. Magnetic induced thermal response curve of unloaded-DOX GYSMNP@PF127 dispersed in water with a concentration of $1.5 \mathrm{mg} \mathrm{mL}^{-1}$ and subjected to an $\operatorname{AMF}\left(\mathrm{f}=340 \mathrm{kHz}\right.$ and $\left.\mathrm{H}=21.0 \mathrm{kA} \mathrm{m}^{-1}\right)$.

After each drug release experiment under AMF, samples were centrifuged and the supernatants (cf. insets of Figure 4.11 (a) and (b)), analysed to determine the amount of DOX released. The results revealed that after 30 min under AMF, just $6.8 \%$ of DOX is released at $\mathrm{pH} 7.4$, while $45.6 \%$ at $\mathrm{pH}$ 4.5. The percentage of DOX released under AMF at $\mathrm{pH} 4.5$ is in the range of that obtained under external heating conditions at $45{ }^{\circ} \mathrm{C}$ in the first $30 \mathrm{~min}$, 
i.e., 44.0\% (Figure 4.10). Even though, under AMF conditions, the temperature reached was slightly lower $\left(40-43^{\circ} \mathrm{C}\right)$ and the length of the test was shorter $(17 \mathrm{~min})$. These better drug release performances observed in AMF can be ascribed to the local heating effects in the vicinity of magnetic nanoparticles, which leads to an increase of the temperature on their surface, without bringing a significant increase in the surrounding medium [30]. Therefore, these results suggest that under an AMF, the local heat generated on the GYSMNP@PF127 surface can help the selective diffusion of loaded DOX into the acidic tumour microenvironment through magnetic hyperthermia. Based on these results, the developed GYSMNP@PF127 material show high promise to act as dual endogenous $\mathrm{pH}$ - and exogenous $\mathrm{AMF} /$ temperature-responsive drug nanocarrier for controlled drug delivery.

\subsubsection{In vitro cell tests}

(i) Haemocompatibility assays

The haemocompatibility of GYSMNP@PF127 was evaluated by haemolysis analysis, ranging the nanocarrier concentration between 10 and $500 \mu \mathrm{gL}^{-1}$ in diluted $\mathrm{RBCs}$ (red blood cells) during $3 \mathrm{~h}$ at $37^{\circ} \mathrm{C}$ (Figure 4.13).

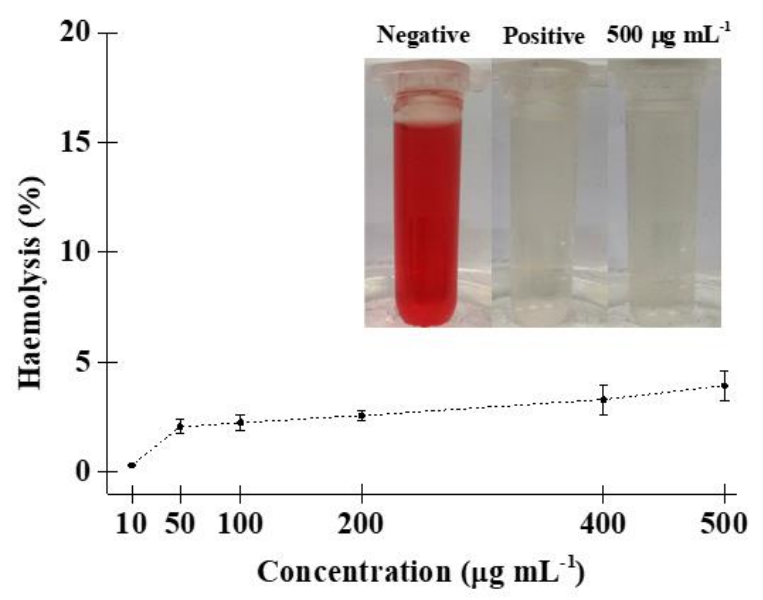

Figure 4.13. Haemolytic percentages of GYSMNP@PF127 at different concentrations ranging from 10 to $500 \mu \mathrm{g} \mathrm{mL} \mathrm{L}^{-1}$ during $3 \mathrm{~h}$ at $37^{\circ} \mathrm{C}$. Error bars represent the standard deviation of triplicate haemolysis tests of two blood donors $(n=6)$.

GYSMNP@PF127 shows a very low dose-dependent haemolytic activity even at a very high concentrations ( $500 \mu \mathrm{g} \mathrm{mL}^{-1}$ ) with less than $4 \%$ of haemolysis, as inferred by the colourless supernatant obtained after $3 \mathrm{~h}$ of incubation at $37^{\circ} \mathrm{C}$ (Figure 4.13). According to the standard ISO 10993-4 that evaluates biological medical devices suitable for blood contact, GYSMNP@PF127 is suitable to be applied directly into the blood stream by exhibiting haemolytic percentages lower than $5 \%[14,46]$. 
(ii) Cell cytotoxicity assays and cellular uptake of drug nanocarriers

The cell biocompatibility of GYSMNP@PF127 and cytotoxicological effect of GYSMNP@PF127-DOX were investigated by SRB colorimetric assay, where the growth inhibition of several concentrations of the nanocarriers $\left(0.05-50 \mu \mathrm{g} \mathrm{m}^{-1}\right)$ were assessed (Figure 4.14). GYSMNP@PF127 shows a good biocompatibility behaviour on healthy liver cells PLP2, even after a long exposition time (48 h) with a GI50 of $58.86 \pm 0.69 \mu \mathrm{g} \mathrm{mL}^{-1}$. To assess GYSMNP@PF127-DOX as drug release nanocarrier, research was carried out in tumour liver cell line, HepG2. Figure 4.14 (b) illustrates a significant drop in the viability of the tumour cells that can be attributed to the increasing concentration of GYSMNP@PF127-DOX, showing a much lower GI50 of $7.34 \pm 3.39 \mu \mathrm{g} \mathrm{mL}^{-1}$ when compared to GYSMNP@PF127. This higher cytotoxicity observed for GYSMNP@PF127-DOX is attributed to the DOX released on the tumour cells.

(a)

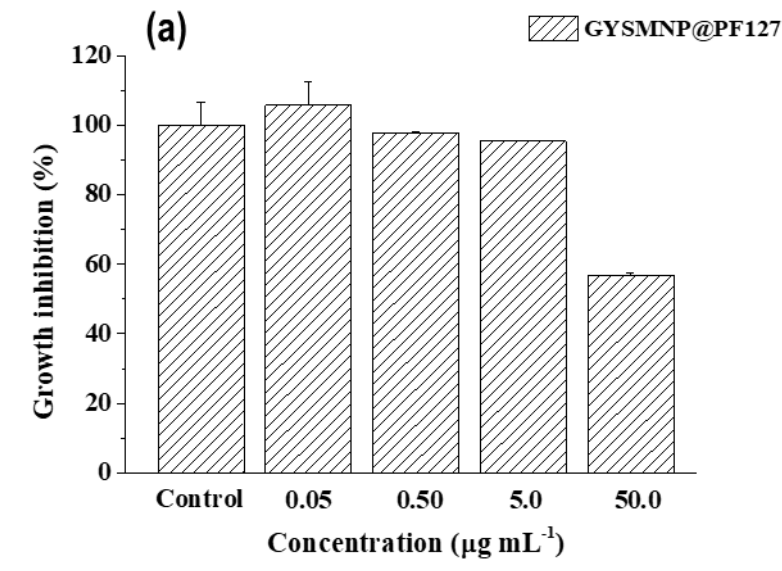

(b)

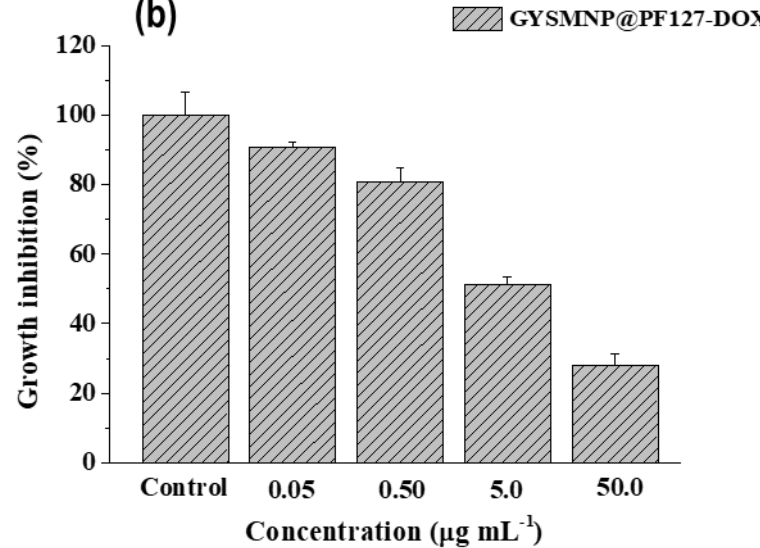

Figure 4.14. Cell cytotoxicity assays investigated by SRB colorimetric assay after incubation of (a) GYSMNP@PF127 in healthy liver cell line PLP2; (b) GYSMNP@PF127-DOX in tumour liver cell line HepG2, under different concentrations for $48 \mathrm{~h}$.

For a better understanding of the therapeutic effect of the DOX released by the GYSMNP@PF127 material over the tumour cells, HepG2 cells were incubated with the drug nanocarrier for 2, 4 and $6 \mathrm{~h}$. In addition, the cell nuclei were stained with Hoechst and microtubules were immunolabeled with a monoclonal anti-a-tubulin antibody (B512) combined with a secondary goat anti-mouse IgG antibody conjugated with Alexa Fluor 488. Cells were observed using confocal microscopy images obtained with Zeiss Laser Confocal Scanning Microscope. Laser at $405 \mathrm{~nm}$ was used to observe the nucleus, $488 \mathrm{~nm}$ to observe the microtubules and $561 \mathrm{~nm}$ to get the imaging from DOX release. It can be observed (Figure 4.15) that the red fluorescence intensity from DOX increases along the incubation time between the nanocarriers and the tumour cells. This observation reveals that the DOX is continuously released from GYSMNP@PF127-DOX nanocarriers. After $6 \mathrm{~h}$, the cell nuclei 
exhibit a strong DOX red fluorescence intensity, suggesting that a high content of DOX is released into the cell cytoplasm and reaches the nucleus after nanoparticle internationalization by endocytosis. The cellular uptake of drug nanocarriers is not only very important for enhancing the drug delivery efficiency, but it is also beneficial for magnetic hyperthermia therapy, allowing a combinatorial thermo-chemotherapeutic effect.

This combined strategy represents an important step forward in the fight against cancer and highlight this new generation of responsive graphene-based nanocarriers as suitable therapeutic actuators on it. Furthermore, their ability to be further functionalized with targeting molecules opens new opportunities for the treatment of different tumour types. Also, the presented magnetic nanocarriers might be optimized with target molecules, for a more effective nanosystem for the in vivo cancer therapy.

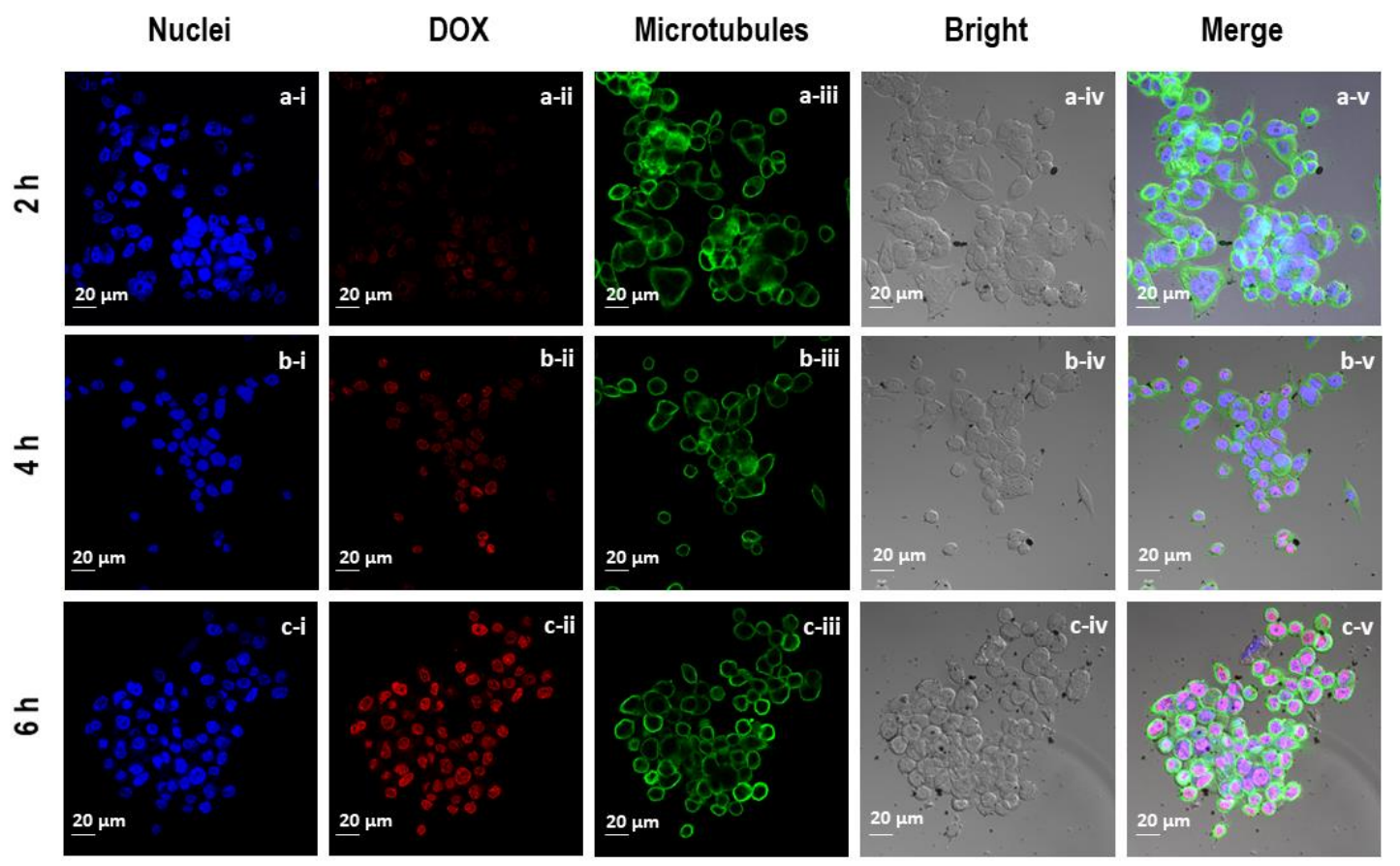

Figure 4.15. Confocal microscopy images of HepG2 cells incubated with GYSMNP@PF127-DOX at a concentration of $5 \mu \mathrm{g} \mathrm{mL}^{-1}$ during $2 \mathrm{~h} \mathrm{(a)}, 4 \mathrm{~h}$ (b) and $6 \mathrm{~h}$ (c). (i) Cell nuclei stained with Hoechst and excited at $405 \mathrm{~nm}$; (ii) DOX released in HepG2 cells and excited at $561 \mathrm{~nm}$; (iii) Cell microtubules immunolabeled with the monoclonal anti-tubulin antibody (B512) combined with a secondary goat anti-mouse lgG antibody conjugated with Alexa Fluor 488 excited at $488 \mathrm{~nm}$; (iv) HepG2 cells using bright contrast; (v) Cell merged images.

\subsubsection{Conclusions}

In this study, hydrophilic graphene-based yolk-shell magnetic nanoparticles (GYSMNP) functionalized with copolymer PF-127, were successfully developed as multifunctional nanocarriers for biomedical applications. To enhance stability and biocompatibility of the graphene-based nanomaterials, samples were chemically activated 
with nitric acid and functionalized with copolymer PF-127. Impressively, this hybrid nanomaterial was found to be an efficient nanoheater at relatively low concentrations, with exceptional drug loading capacity and controlled drug release triggered by the acidic tumour microenvironment. In addition, the external application of an AMF (magnetic hyperthermia) was observed to further induce drug release, which was more significant at the lowest $\mathrm{pH}$ tested. The dual $\mathrm{pH}$ - and temperature-responsive drug delivery behaviour observed in these nanosystems show a burst release of the drug at mimicked acidic tumour environment, whereas at physiological $\mathrm{pH}$ and temperature, negligible amount of drug is released, confirming that the drug is efficiently retained in the nanocarrier. Furthermore, the developed nanocarriers show low cytotoxicity and nuclei drug internalization in a short period of time. The presented strategy can represent a new way to design and synthesize stable graphene-based materials with novel structures for targeted combinatorial thermochemotherapy triggered by abnormal acidic cell microenvironments on tumour site, with the subsequent minimization of side effects on the healthy cells. 


\section{References}

[1] A. R. K. Sasikala, R. G. Thomas, A. R. Unnithan, B. Saravanakumar, Y. Y. Jeong, C. H. Park and C. S. Kim, Scientific Report, 2016, 6, 20543.

[2] X. Huang, S. Wu and X. Du, Carbon, 2016, 101, 135-142.

[3] R. Tietze, J. Zaloga, H. Unterweger, S. Lyer, R. P. Friedrich, C. Janko, M. Pöttler, S. Dürr and C. Alexiou, Biochemical and Biophysical Research Communications, 2015, 468, 463-470.

[4] Z. Li, E. Ye, David, R. Lakshminarayanan and X. J. Loh, Small, 2016, 12, 4782-4806.

[5] S. Mura, J. Nicolas and P. Couvreur, Nature Materials, 2013, 12, 991-1003.

[6] D. Chen, C. A. Dougherty, K. Zhu and H. Hong, Journal of Controlled Release, 2015, 210, 230-245.

[7] V. V. Mody, A. Cox, S. Shah, A. Singh, W. Bevins and H. Parihar, Applied Nanoscience, 2014, 4, 385-392.

[8] S. Mohapatra, S. R. Rout, R. K. Das, S. Nayak and S. K. Ghosh, Langmuir, 2016, 32, 16111620.

[9] S. Li, J. Zheng, D. Chen, Y. Wu, W. Zhang, F. Zheng, J. Cao, H. Ma and Y. Liu, Nanoscale, 2013, 5, 11718-11724.

[10] W. S. Hummers and R. E. Offeman, Journal of the American Chemical Society, 1958, 80, 1339.

[11] M. Sarno, C. Cirillo, C. Scudieri, M. Polichetti and P. Ciambelli, Industrial \& Engineering Chemistry Research, 2016, 55, 3157-3166.

[12] S. Chandra, G. Noronha, S. Dietrich, H. Lang and D. Bahadur, Journal of Magnetism and Magnetic Materials, 2015, 380, 7-12.

[13] Y. Wang, R. Zhao, S. Wang, Z. Liu and R. Tang, Biomaterials, 2016, 75, 71-81.

[14] R. O. Rodrigues, M. Bañobre-López, J. Gallo, P. B. Tavares, A. M. T. Silva, R. Lima and H. T. Gomes, Journal of Nanoparticle Research, 2016, 18, 1-17.

[15] W.-J. Liu, Y.-X. Liu, X.-Y. Yan, G.-P. Yong, Y.-P. Xu and S.-M. Liu, Journal of Materials Chemistry A, 2014, 2, 9600-9606.

[16] R. S. Ribeiro, Z. Frontistis, D. Mantzavinos, D. Venieri, M. Antonopoulou, I. Konstantinou, A. M. T. Silva, J. L. Faria and H. T. Gomes, Applied Catalysis B: Environmental, 2016, 199, 170-186.

[17] R. M. V. Abreu, I. C. F. R. Ferreira, R. C. Calhelha, R. T. Lima, M. H. Vasconcelos, F. Adega, R. Chaves and M.-J. R. P. Queiroz, European journal of medicinal chemistry, 2011, 46, 5800-5806.

[18] B. Svobodova, L. Barros, R. C. Calhelha, S. Heleno, M. J. Alves, S. Walcott, M. Bittova, V. Kuban and I. C. F. R. Ferreira, Industrial Crops and Products, 2017, 95, 365-373.

[19] L. M. Pastrana-Martínez, S. Morales-Torres, V. Likodimos, P. Falaras, J. L. Figueiredo, J. L. Faria and A. M. T. Silva, Applied Catalysis B: Environmental, 2014, 158-159, 329340.

[20] A. C. Ferrari, J. C. Meyer, V. Scardaci, C. Casiraghi, M. Lazzeri, F. Mauri, S. Piscanec, D. Jiang, K. S. Novoselov, S. Roth and A. K. Geim, Physical Review Letters, 2006, 97, 187401.

[21] A. C. Ferrari and D. M. Basko, Nature Nanotechnology, 2013, 8, 235-246. 
[22] A. C. Ferrari and J. Robertson, Physical Review B, 2000, 61, 14095-14107.

[23] A. Bianco, H.-M. Cheng, T. Enoki, Y. Gogotsi, R. H. Hurt, N. Koratkar, T. Kyotani, M. Monthioux, C. R. Park, J. M. D. Tascon and J. Zhang, Carbon, 2013, 65, 1-6.

[24] A. Kaniyoor and S. Ramaprabhu, AIP Advances, 2012, 2, 032183.

[25] V. C. Sanchez, A. Jachak, R. H. Hurt and A. B. Kane, Chemical Research in Toxicology, 2012, 25, 15-34.

[26] B. Zhang, Y. Wang and G. Zhai, Materials Science and Engineering C, 2016, 61, 953964.

[27] A. Sasidharan, L. S. Panchakarla, P. Chandran, D. Menon, S. Nair, C. N. R. Rao and M. Koyakutty, Nanoscale, 2011, 3, 2461-2464.

[28] X. Yu and Y. Zhu, Science and Technology of Advanced Materials, 2016, 17, 229-238.

[29] J. Liu, Z. Luo, J. Zhang, T. Luo, J. Zhou, X. Zhao and K. Cai, Biomaterials, 2016, 83, 51-65.

[30] A. Hervault, A. E. Dunn, M. Lim, C. Boyer, D. Mott, S. Maenosono and N. T. K. Thanh, Nanoscale, 2016, 8, 12152-12161.

[31] S. Li, J. Guo, R. A. Patel, A. L. Dadlani and R. M. Leblanc, Langmuir, 2013, 29, 57425748.

[32] A. Pitto-Barry and N. P. E. Barry, Polymer Chemistry, 2014, 5, 3291-3297.

[33] Q. Zhou, Z. Zhang, T. Chen, X. Guo and S. Zhou, Colloids and Surfaces B: Biointerfaces, 2011, 86, 45-57.

[34] A. Meshkini and H. Oveisi, Colloids and Surfaces B: Biointerfaces, 2017, 158, 319-330.

[35] G. M. Mutlu, G. R. S. Budinger, A. A. Green, D. Urich, S. Soberanes, S. E. Chiarella, G. F. Alheid, D. R. McCrimmon, I. Szleifer and M. C. Hersam, Nano Letters, 2010, 10, 1664-1670.

[36] J. Siepmann and F. Siepmann, International Journal of Pharmaceutics, 2008, 364, 328343.

[37] X. Zhang, Y. Wang, Y. Zhao and L. Sun, Materials Science and Engineering C, 2017, 77, 19-26.

[38] A. Monks, D. Scudiero, P. Skehan, R. Shoemaker, K. Paull, D. Vistica, C. Hose, J. Langley, P. Cronise, A. Vaigro-Wolff, M. Gray-Goodrich, H. Campbell, J. Mayo and M. Boyd, Journal of the National Cancer Institute, 1991, 83, 757-766.

[39] W. Voigt, in Chemosensitivity: In Vitro Assays, ed. R. D. Blumenthal, Humana Press, Totowa, NJ, 2005, pp. 39-48.

[40] S. A. C. Lima, A. Gaspar, S. Reis and L. Durães, Materials Science and Engineering C, 2017.

[41] I. Pradhan, R. Srivastava and D. Bahadur, Acta Biomaterialia, 2014, 10, 2976-2987.

[42] P. Mohan and N. Rapoport, Molecular Pharmaceutics, 2010, 7, 1959-1973.

[43] M. Mahdavi, F. Rahmani and S. Nouranian, Journal of Materials Chemistry B, 2016, 4, 7441-7451.

[44] Y. Oh, M. S. Mooethy, P. Manivasagan, S. Bharathiraja and J. Oh, Biochimie, 2017, 133, 7-19.

[45] P. Presa, Y. Luengo, M. Multigner, R. Costo, M. P. Morales, G. Rivero and A. Hernando, The Journal of Physical Chemistry C, 2012, 116, 25602-25610. 
[46] Q. Wang, M. Shen, T. Zhao, Y. Xu, J. Lin, Y. Duan and H. Gu, Scientific reports, 2015, 5,7774 . 



\section{CHAPTER V \\ Organ-on-a-chip platform for bio-toxicity evaluation of graphene-based magnetic nanoparticles}

5.1 Introduction

5.2 Dual-organ-on-a-chip platform developed for drug screening studies in nanomedicine 



\section{Organ-on-a-chip platform for bio-toxicity evaluation of graphene-based magnetic nanoparticles}

\subsection{Introduction}

In spite of all the progress achieved in the last decade in nanomedicine, the translation of new therapeutic nanosystems for clinical application has been slow, especially due to the lack of robust preclinical tissue culture platforms able to mimic the in vivo conditions found in the human body and to predict the performance of the developed nanomaterials [1].

Organ-on-a-chip platforms are novel microfluidic tools that mimic complex human organ functions at the microscale level. These integrated microfluidic networks, with 3D tissue engineered models, have shown the ability to reduce the discrepancies between the preclinical and clinical trials [1, 2]. Moreover, these 3D microfluidic platforms have several advantages over the in vivo animal models, such as lower costs and less time-consuming, ethical concerns, visualization of the theranostic agents in the target tissues and accuracy to predict human responses [1].

In this Chapter, a novel organ-on-a-chip platform to evaluate with high accuracy the performance of new drug nanocarriers, such as the developed GbMNP, as intelligent and ondemand drug delivery system with $\mathrm{pH}$-dependent controlled release, is described. This work was developed under the framework of a Fulbright Research Grant 2017, at the Harvard-MIT Division of Health Sciences and Technology (Cambridge, Massachusetts, USA), headed by Prof. Ali Khademhosseini and supervised by Dr Su Ryon Shin. This bioengineering leading group has a vast experience in developing organ-on-a-chip systems that aims to mimic human response to various chemicals.

In Section 5.2, part of the study to design and test a dual-organ-on-a-chip platform, compressing cardiac and breast cancer organoids, with the aim to evaluate the effect of chemotherapy over those organs in continuous and during several days, is detailed. Due to the versatility of the proposed $3 \mathrm{D}$ tissue/organ platform, the evaluation of novel drug nanocarriers, such as the GYSMNP@PF127-DOX developed in Section 4.3, was assessed.

A manuscript is under preparation with the complete study, and it will be submitted to an international journal with high reputation in the field, expected to be a new avenue for the development of 3D screening platform for drug delivery and nanomedicine. 


\subsection{Dual-organ-on-a-chip platform developed for drug screening studies in nanomedicine}

Among the 8.2 million cancer deaths occurred in 2012 worldwide, breast cancer is the leading cause of cancer-related death in females [3], and the most common cancer diagnosed in women in North America, Europe and Oceania [4]. Also, it is estimated that without major changes in prevention or treatment, more than 846000 women will die in 2035 from breast cancer worldwide [5].

Chemotherapy is a vital part of the frontline attack of cancer, including breast cancer. But, although chemotherapeutic drugs have successfully treated many patients, the benefits of chemotherapy are tempered by its significant limitations. One of the most worrying limitations is the non-specific targeting that chemotherapeutic drugs have over the tumour cells, affecting also the healthy ones [6]. Among the side effects, cardiac toxicity is gaining particular attention due to its high rate of occurrence. The term cardiotoxicity includes many possible pathological manifestations, but the most common is the reduction of the cardiac function, which can lead to heart failure and death [7]. Additionally, it has been estimated that about $30 \%$ of the drug candidates fail during clinical trials, due to its toxic side effects, which generates major costs to the pharmaceutical industry and slows down the drug development process [8]. This is partly due to the fact that many animal models fail to accurately reflect human physiology. Thus, there is an increasing need to develop human-based tissue/organ models to mimic the architecture and functionality of human tissues for drug toxicity assays before clinical trials [9].

Another major limitation of chemotherapy is the physical nature of tumours, which blocks the penetration of drugs into the tumour tissues [10]. To address this issue, researchers have been pursuing ways to develop new systems, such as nanocarriers, able to increase the amount of drug internalized on cells after these nanosystems have reached their targets. Nevertheless, the translation of new therapeutic nanosystems for clinical applications has been also hampered by the lack of accurate 3D human models able to mimic the architecture and functionality of their respective counterparts.

Organs-on-chips are advanced microfluidic devices able to miniaturize the complex 3D architecture and to reproduce the biomimetic stimuli, both dynamic mechanical and chemical, found in human tissues and organs [11, 12]. Due to their features, these in vitro models have emerged as viable platforms for personalized medicine, drug screening and nanomedicine studies [12, 13]. An additional advantage of these in vitro models is the possibility to integrate, in the same platform, multiple organoid models connected in a 
similar manner as they are arranged in vivo, and thus able to analyse multi-organ interactions [12]. In spite of the several organ-on-a-chip models developed in the recent years, the integration of various organs has been limited due to the successful integration of multisensor systems. These multisensory systems play a crucial role in the continuous monitoring of the organ models in response to the drugs or therapeutic nanosystems tested over an extended period of time.

In this context, the goal of this chapter is to address these challenges by developing a dualorgan-on-a-chip platform, comprising human breast cancer and heart-on-a-chip, and equipped with a monitoring system that allows for long-term, the accurate measurement of biomarkers secreted by both tissues in response to free or encapsulated chemotherapeutic drugs.

To accomplish this goal, herein is proposed an innovative approach that is based on a labelfree electrochemical (EC) biosensor in conjunction with human breast cancer-and-heart-ona-chip technology. The biosensor uses EC impedance spectroscopy which allows the measurement of a variety of bioassays with high sensitivity, as recently demonstrated by the Khademhosseini's group [9, 12]. The microfabricated EC biosensors are amenable to scaling and allow synergistic integration of the sensors with a microfluidic platform. The EC sensor arrays can be functionalized with aptamers/antibodies for multiplexed detection of biomarkers secreted from both dual cancer-and-heart-on-a-chip model. The extended aim of the proposed biosensing platform is to be further equipped with a built-in capability of regenerating the sensor surface upon saturation for continual kinetic studies over extended periods of time, by combining it with an automated microfluidic system (work that is expected to be soon published). Particularly, in this section, the chemotherapeutic drug DOX is evaluated in situ via quantification of selected biomarkers, secreted by the cancer and human cardiac models. Additionally, the GYSMNP@PF127-DOX nanocarrier, developed in Section 4.3, is evaluated against the free DOX in order to assess its bio-toxicity and drug release efficiency on both cancer and healthy organoids. This novel in vitro platform can represent a potential avenue to promote the development of a highly efficient and robust preclinical tissue culture platform, able to achieve better prediction of efficiency/toxicity of drugs and nanomaterials developed for nanomedicine. 


\subsubsection{Experimental methods}

\subsubsection{Chemicals}

Potassium ferricyanide, $\mathrm{K}_{2} \mathrm{Fe}(\mathrm{CN})_{6}$ (99 wt.\%), sulfuric acid, $\mathrm{H}_{2} \mathrm{SO}_{4}$ (95 wt.\%), Dulbecco's phosphate buffered saline (DPBS), 11-mercaptoundecanoic acid, 11-MUA (95 wt.\%), ethanol absolute, $\mathrm{C}_{2} \mathrm{H}_{6} \mathrm{O}$ (99.8 wt.\%), N-ethylcarbodiimide, EDC (97 wt.\%), and $\mathrm{N}$-hydroxysuccinimide, NHS (97 wt.\%) were obtained from Sigma-Aldrich (MO, USA). Doxorubicin hydrochloride, DOX (98 wt. \%), was purchased from Discovery Fine Chemicals (Wimborne, UK). Polydimethylsiloxane (PDMS) and curing agent were obtained from Sylgard 184, Dow Corning (MI, USA). PrestoBlue cell viability assay was obtained from ThermoFisher scientific (MA, USA). Live/Dead cell viability assays were purchased from Invitrogen (CA, USA). Human creatine kinase (CKMB), cardiac Troponin $T$ and Human Epidermal growth factor Receptor 2 (HER2) ELISA Kits were purchased from Abcam (Cambridge, UK). All aqueous solutions were prepared using ultrapure water $\left(18.2 \mathrm{M} \Omega . \mathrm{cm}\right.$ at $\left.25{ }^{\circ} \mathrm{C}\right)$, produced in a Milli-Q system (Millipore), or deionized water.

\subsubsection{Preparation of microelectrodes}

Single and multi-array microelectrodes were designed and fabricated using the same procedure reported elsewhere [9]. Briefly, the microelectrode set was fabricated with a reference electrode (RE), a counter electrode (CE) and a working electrode (WE), and produced in gold $(\mathrm{Au})$ to achieve a stable biosensing system. The WE diameter is $800 \mu \mathrm{m}$, the CE and RE width are $150 \mu \mathrm{m}$, the gap between the WE and the other two electrodes is $200 \mu \mathrm{m}$, and the diameter of detection area is about $1500 \mu \mathrm{m} .20 \mathrm{~nm}$ thick titanium (Ti), 20 $\mathrm{nm}$ thick palladium (Pd) and $500 \mathrm{~nm}$ thick Au were selectively deposited on cleaned glass using a shadow mask by an e-bean evaporator, creating WE and CE electrodes. A second shadow mask for RE patterning was attached to the Au-deposited glass subtract and $20 \mathrm{~nm}$ thick Ti, $20 \mathrm{~nm}$ thick Pd and $500 \mathrm{~nm}$ thick silver (Ag) were selectively deposited on the glass subtract. Lastly, the electrodes were annealed at $300^{\circ} \mathrm{C}$ for $6 \mathrm{~h}$ in a furnace.

\subsubsection{Electrochemical measurements}

Measurements of electrochemical impedance spectroscopy (EIS) were performed using an electrochemical workstation $\mathrm{CHI660 \textrm {E }}$ ( $\mathrm{CH}$ instrument, Inc.), with a conventional three electrode system. For the EIS technique, the initial potential was set to $0.1 \mathrm{~V}$ and the 
frequencies ranging between $0.1 \mathrm{~Hz}$ and $100 \mathrm{kHz}$ at $5 \mathrm{mV}$ of amplitude. All measurements were carried out in $50 \mathrm{mM}$ of potassium ferricyanide $\left(\mathrm{K}_{2} \mathrm{Fe}(\mathrm{CN})_{6}\right)$ electrolyte solution.

\subsubsection{Design and fabrication of dual bioreactor chips}

The drawing of the dual bioreactor was performed with the software Solidworks and the PMMA mask produced with a laser cutting machine. To fabricate the PDMS mould, the PMMA mask was covered with PDMS prepared by mixing curing agent and base polymer $(1: 10, \mathrm{w} / \mathrm{w})$, and the mixture cured in the oven at $80^{\circ} \mathrm{C}$ for $1 \mathrm{~h}$. The cured PDMS mould was peeled off from the mask. For each dual bioreactor chip, two pieces of the cured PDMS containing the dual-organ geometry were used. Finally, the PDMS moulds were placed between two PMMA plates and fixed with 8 screws to avoid leakages and contaminations, as shown in Figure 5.1.

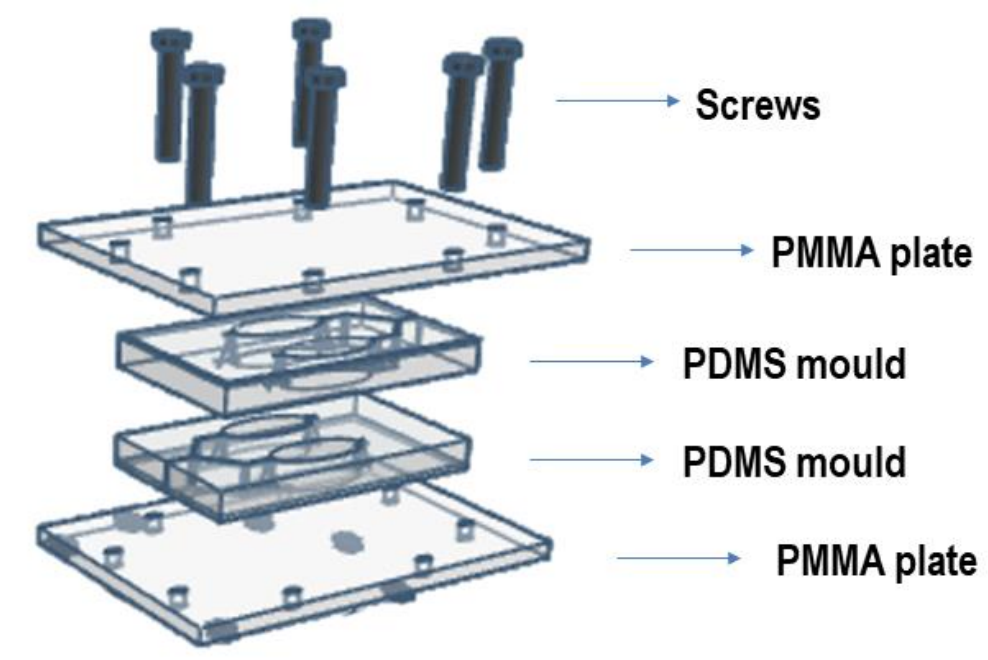

Figure 5.1. Schematic representation of the dual bioreactor chip used as microfluidic platform to contain the dual-organ system.

\subsubsection{Immobilization process for aptamer biosensors}

The immobilization process of the selected aptamers (cf. Table 5.1) in the biosensors was performed as described elsewhere [9]. Two cardiac biomarkers (CKMB and Troponin T), and one breast cancer biomarker (HER2) were exploited. Briefly, prior to selfassembly monolayer (SAM) coating, the fabricated microelectrodes were first cleaned using $\mathrm{H}_{2} \mathrm{SO}_{4}(10 \mathrm{mM})$ and DPBS solutions three times. Then, the microelectrodes were coated with $30 \mu \mathrm{L}$ of 11 -mercaptoundecanoic acid (11-MUA) solution $(10 \mathrm{mM})$ for $1 \mathrm{~h}$ to generate a thiol terminated SAM. The microelectrodes were rinsed with ethanol and DPBS to remove the unbounded 11-MUA molecules. In the following step, $60 \mu \mathrm{L}$ of the mixed solution of $\mathrm{N}$ ethylcarbodiimide (EDC) (50 mM) and $\mathrm{N}$-hydroxysuccinimide (NHS) (50 mM) was applied to 
the electrode surface and incubated for $15 \mathrm{~min}$. In this process, the activated NHS ester reacts with the primary amine groups of aptamers by the formation of an amide bond. Then, the selected aptamer $\left(10 \mu \mathrm{g} \mathrm{mL}^{-1}\right)$ was incubated for $30 \mathrm{~min}$ and the unbounded molecules rinsed with DPBS. The unspecific sites were blocked with cell culture media for 30 min, which also served as baseline. Finally, the antigens of the selected biomarkers were measured after flowed into the biosensors and being incubated for $30 \mathrm{~min}$. The same procedure was used to perform the calibration curves of the selected biomarkers. In this case, different concentrations of the antigens $\left(0.1 \mathrm{pg} \mathrm{mL}^{-1}\right.$ to $\left.1 \mathrm{ng} \mathrm{mL}^{-1}\right)$ were successively incubated for $30 \mathrm{~min}$ and the Nyquist curves obtained. Then, to eliminate any electrochemical difference that could exist among the different electrodes, the electrontransfer resistance $\left(R_{c t}\right)$ was normalized as $R_{c t}$ antigen/ $R_{c t}$ media. Each experiment was performed in triplicate using independent biosensors.

Table 5.1. Selected cardiac and breast cancer biomarkers and their clinical cut-off levels

\begin{tabular}{cccc}
\hline Tissue & Biomarker & $\begin{array}{c}\text { Clinical cut-off levels } \\
(\mathrm{ng} / \mathrm{mL})\end{array}$ & Specificity \\
\hline Cardiac & Troponin T & $0.05-0.1$ & High \\
& CKMB & 0.6 & Medium \\
Breast cancer & HER2 & $8.1-75$ & High \\
\hline
\end{tabular}

\subsubsection{In vitro 2D cellular studies using graphene-based magnetic nanoparticles (GYSMNP)}

Prior to the microfluidic bioreactor study using the organ-on-a-chip platform, the drug nanocarrier was tested in in vitro cellular 2D studies. These studies allowed to: (i) obtain the optimal concentration of the drug nanocarrier loaded with DOX (GYSMNP@PF127-DOX) in order to deliver equal amount of the free chemotherapeutic drug (DOX, $10 \mu \mathrm{M}$ ); (ii) determine the biocompatibility of unloaded GYSMNP@PF127 in healthy induced pluripotent stem cardiomyocytes (iPSCs) and breast adenocarcinoma cell lines (SkBr3); and (iii) determine the toxicological effect of the GYSMNP@PF127-DOX on this same cell lines.

(i) Determination of optimal concentration of drug nanocarrier

The optimal concentration of GYSMNP@PF127-DOX to release equal amount of free DOX $(10 \mu \mathrm{M})$ was verified by ranging the drug nanocarrier concentrations between 15 and $60 \mu \mathrm{g} \mathrm{mL}{ }^{-1}$, which correspond to a total loaded DOX of 10 and $40 \mu \mathrm{M}$. SkBr3 cell line was 120 
used to assess the cellular uptake of drug delivery by the nanocarriers. Briefly, $100 \mu \mathrm{L}$ of McCoy's 5a medium modified containing different concentrations of GYSMNP@PF127-DOX, was seeded in 96-well plates containing the cell line $\mathrm{SkBr} 3$, at an initial cell density of $1.0 \times 10^{4}$. In addition, control wells containing just cells with cultured medium were seeded and used as blank. On another well seeded with cells, free DOX was added at $10 \mu M$, to be used as comparison drug control. All the assays were performed in two individual tissueculture plates in duplicated wells $(n=4)$ and cultured in $5 \% \mathrm{CO}_{2}$, at $37^{\circ} \mathrm{C}$ for $24 \mathrm{~h}$.

(ii) Biocompatibility and toxicological studies of drug nanocarrier

The biocompatibility and toxicological studies of GYSMNP@PF127 and GYSMNP@PF127-DOX were conducted in cardiomyocytes and $\mathrm{SkBr} 3$ cell lines using PrestoBlue colorimetric assay. Briefly, $100 \mu \mathrm{L}$ of McCoy's 5a medium modified containing unloaded GYSMNP@PF127 (16.6 $\left.\mu \mathrm{g} \mathrm{mL}^{-1}\right)$, GYSMNP@PF127-DOX $\left(30 \mu \mathrm{g} \mathrm{mL}{ }^{-1}\right)$ and free DOX $(10 \mu \mathrm{M})$, were seeded in 96-well plates containing the respective organoid cell lines in test, at an initial cell density of $1.0 \times 10^{4}$. After $24 \mathrm{~h}$ (day 1 ), $30 \mu \mathrm{L}$ of PrestoBlue was added to each well and incubated for $30 \mathrm{~min}$ in $5 \% \mathrm{CO}_{2}$, at $37{ }^{\circ} \mathrm{C}$ in dark. The supernatant was collected and used to read the absorbance at 570 and $600 \mathrm{~nm}$. The procedure was repeated in day $3(72 \mathrm{~h})$ and day $5(120 \mathrm{~h})$. In addition, control wells, containing just cells with cultured medium, were seeded and used as control. All the assays were performed in two individual tissue-culture plates in duplicated wells $(n=4)$.

\subsubsection{Culturing cardiomyocytes and breast cancer organoids in microfluidic bioreactor}

A high-throughput PDMS micro-well technique was used to form breast cancer spheroids (Figure 5.2, a-c). A negative silicon mould containing pillars (300 $\mu \mathrm{m}$ in diameter and $250 \mu \mathrm{m}$ in depth) was used to form a PDMS replica against the silicon wafer by casting the PDMS in a mixture of 10:1 silicon elastomer and the curing agent (Sylgard 184, Dow Corning, MI, USA). The blend was then degassed and cured for $1 \mathrm{~h}$ at $80^{\circ} \mathrm{C}$, and the PDMS mould formed. Micro-wells were washed with $70 \%$ ethanol under the vacuum to remove all the micro-bubbles. After trypsinization, $\mathrm{SkBr} 3$ cells were seeded at a concentration of $1 \times 10^{6}$ cells in $1 \mathrm{~mL}$ media (DMEM $1 \mathrm{X}$ medium with $10 \% \mathrm{FBS}$ and $1 \%$ penicillin/streptomycin) per PDMS mould containing $24 \times 24$ micro-wells. The cells were allowed to settle in the wells by gravity for $30 \mathrm{~min}$. The cells that were not settled into the micro-wells were washed with media (Figure 5.2 (g)) several times. The cells were kept in incubation with a daily supply of fresh media for 5 days until spheroids were formed. On day 5 , spheroids were harvested 
and collected for encapsulation in gelatin methacryloyl (GelMA), generating the organoid architecture.

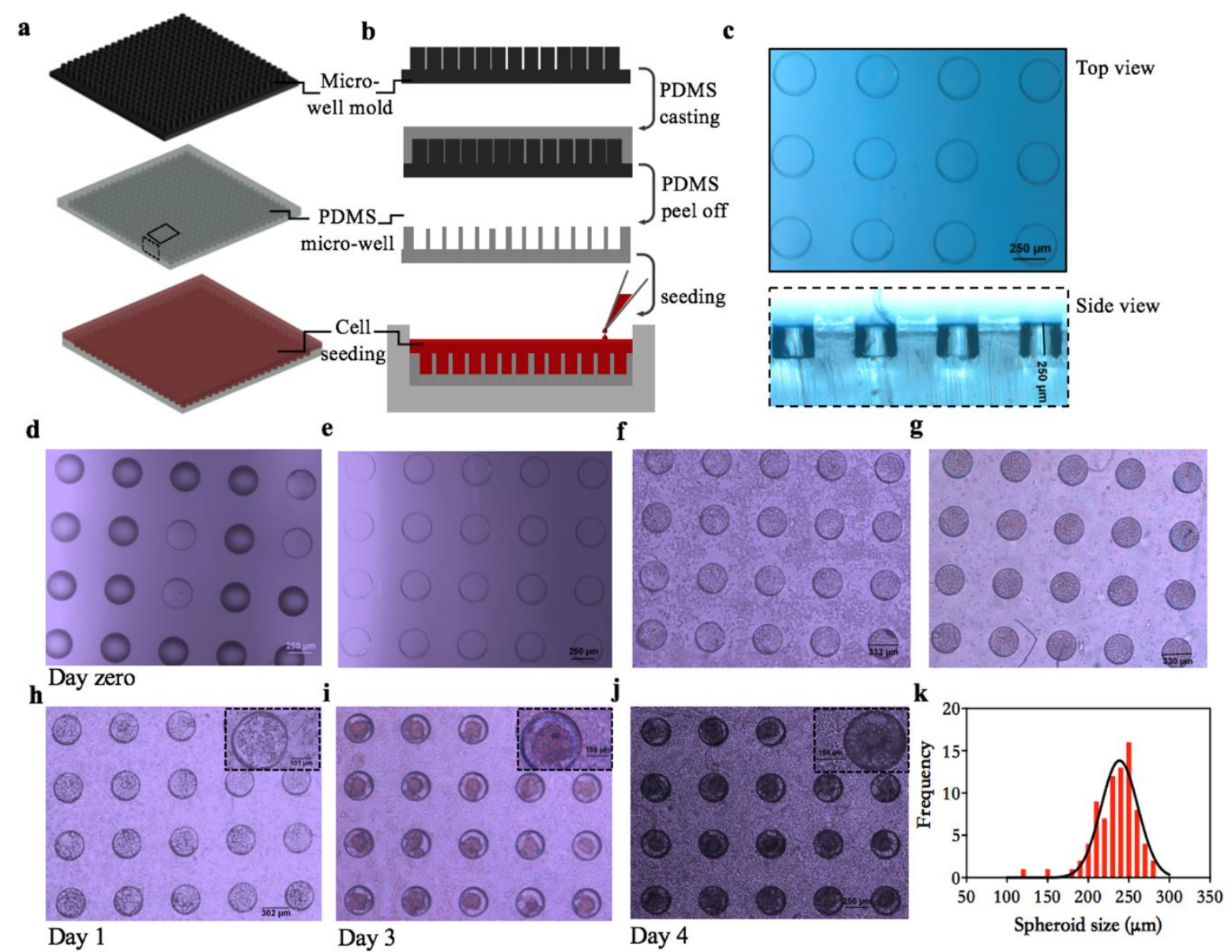

Figure 5.2. (a, b) Schematic representation of the fabrication of PDMS micro-well mould for cancer cell spheroid preparation; (c) optical images of cross-sectional and top view of PDMS micro-well moulds. (d-j) Optical images of PDMS micro-wells mould at different steps: (d) after adding cell media and formation of micro-air bubbles; (e) after vacuum-removing of air bubbles; (f) seeded with $\mathrm{SkBr} 3$ cells $\left(1 \times 10^{6}\right.$ cells $\left.\mathrm{ml}^{-1}\right)$ and incubated for $30 \mathrm{~min}$ at $37^{\circ} \mathrm{C}$ with $5 \% \mathrm{CO}_{2}$; $(\mathrm{g})$ washed with cell media to remove cells that did not settled into the micro-wells; and ( $h-j)$ changed cell media after 24,72 and $96 \mathrm{~h}$ incubation at $37^{\circ} \mathrm{C}$ with $5 \% \mathrm{CO}_{2}$, respectively. Insets show higher magnification of cancer spheroid morphologies; (k) histogram shows SkBb3 spheroid size distribution at day 4 (number of considered spheroids $=100$ ).

Cardiomyocytes and $\mathrm{SkBr} 3$ spheroids were individually embedded inside a GelMA hydrogel, dispensed, and photo-cross-linked to generate the human cell-based dual heart- and breast cancer-on-chip platform. The cardiac and breast organoids were cultured for up to 5 days in the bioreactors and the media used to monitor the amount of biomarkers secreted during the experiments (free DOX and GYSMNP@PF127-DOX). The viability of the cardiomyocytes and breast cancer were further analysed using a live/dead assay. The statistical analysis was performed with the Student's $t$-test with a significance level of $p<0.05$, represented as 
asterisks $\left({ }^{*}\right)$ in comparison with the control test. All statistical analysis were performed using Origin 9.0 software

\subsubsection{Results and discussion}

\subsubsection{Design and calibration of a label-free EC sensing method}

To monitor selected biomarkers, EIS measurements were used. Based on previous studies published by the Khademhosseini's group (showing the high selectivity and stability of gold microelectodes to detect CKMB aptamers/antibodies [9, 14]), the same microelectrode set having a reference electrode (RE), a counter electrode (CE) and a working electrode (WE) (cf. Figure 5.3 (a-b), was tested to be applied as multiplexed labelfree EC sensing. The main goal is to design and fabricate, in a near future, a multiplexed biosensing method able to be further integrated with a microfluidic sensing platform and to measure for long periods of time up to 16 selected biomarkers secreted from different organoids.

Although the recent advances in organ-on-a-chip platforms, the monitoring of biomarkers secreted from organoids in a non-invasive manner is still very challenging. Among those challenges are the limited sensitivity and selectivity to detect selected biomarkers that are dissolved in complex biological environments, such as cell culture medium, and also detect trace amounts of those secreted disease markers [15], such as the case of cardiac CKMB, troponins or HER2 breast cancer biomarkers. The current gold standard to measure these biomarkers relies on ELISA kits, which have insufficient sensitivity to detect trace amounts of biomarkers ( $\mathrm{pg} \mathrm{mL}^{-1}$ ) and requires significant sample volume. EIS based sensors have shown some advantages to overcome these drawbacks, such as low limit-of-detection (LOD), low sample requirements, compatibility with microfluidic techniques and possibility to be integrated with organ-on-a-chip platforms for continuous monitoring [9].

Figure 5.3 (c) represents the immobilization steps of aptamers into the gold surface of the microelectrode. The first step starts with the immobilization of a self-assembling monolayer (SAM) of 11-mercaptoundecanoic acid (11-MUA) solution, containing thiol groups in one end and carboxyl groups in the other terminal.

Then, by mixing EDC/NHS, the carboxyl-terminated alkyl surface of SAM is converted to an active NHS ester by reacting with 11-MUA. In this process, the activated NHS ester reacts with the primary amine groups of the aptamers by the formation of an amide bond. Aptamers, which are short single-stranded oligonucleotides capable of binding molecules 
with high affinity and specificity [16], were used in this study to target specific antigens (troponin-T, CKMB and HER2), in a highly reproducible manner.

(a)

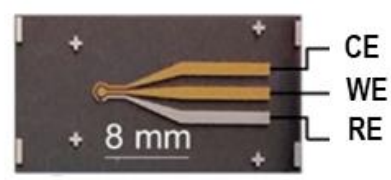

(b)

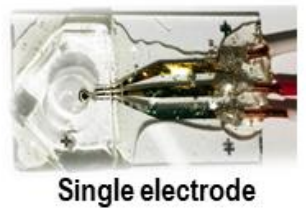

(c)
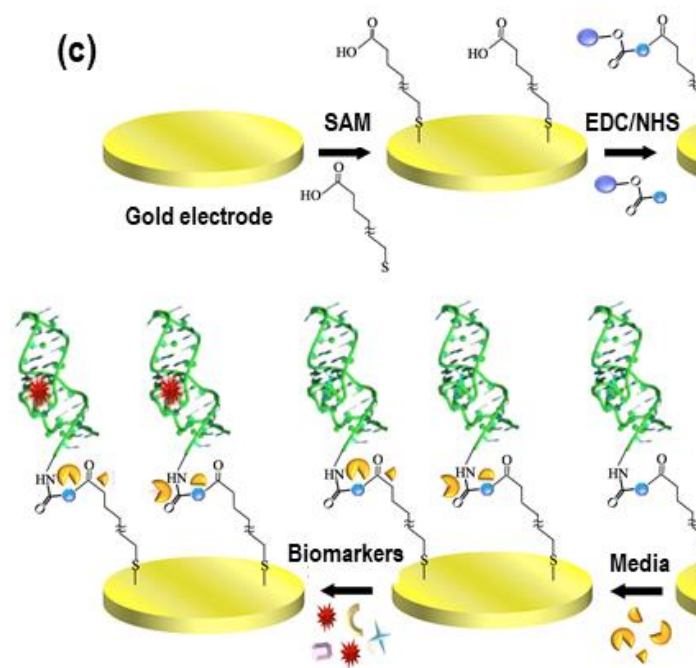

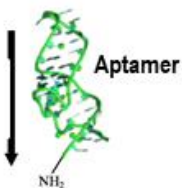

Figure 5.3. (a) Image of the microfabricated electrode set containing a reference electrode (RE), a counter electrode (CE) and a working electrode (WE); (b) image of a single electrode as the one used in this study; (c) schematic diagram of the immobilization steps of the aptamers into the microelectrode.

Cardiac troponin biomarkers represent a promising and valuable modality for detecting cardiac toxicity in patients undergoing chemotherapy. Monitoring cardiac troponin levels provide information regarding the development of cardiac toxicity long before cardiac dysfunction becomes apparent on echocardiography or via clinical symptoms.

CKMB is another cardiac biomarker related to recurrence of myocardial infarction and cardiac vascular disease [17]. The low clinical cut-off level of this biomarker $\left(0.6 \mathrm{ng} \mathrm{mL}^{-1}\right)$ is a critical concern while selecting the suitable sensors. Therefore, a highly sensitive biosensor is required for in-line detection of secreted CKMB from the heart-on-a-chip platform, which can be employed for real-time monitoring of cardiotoxicity induced by drugs.

On the other hand, the expression level of human epidermal growth factor receptor HER2 increases in approximately $15-30 \%$ in breast cancer tumours [18]. For instance, breast cancer patients who are positive to HER2 have increased HER2 concentrations in blood (15-75 $\left.\mathrm{ng} \mathrm{mL} \mathrm{m}^{-1}\right)$ compared to normal individuals $\left(2-15 \mathrm{ng} \mathrm{mL}^{-1}\right)$ [19]. Moreover, HER2 overexpression has also been seen in other cancers like ovary, endometrium, bladder, lung, colon, and head and neck [18]. Thus, HER2 valuation may be of clinical relevance especially in the development of breast cancer-on-a-chip platforms for the evaluation of chemotherapeutic drugs. Due to these reasons, these biomarkers were selected to monitor the response of organoids to chemotherapy administrated via free or encapsulated DOX. 
The calibration and sensitivity of the biosensors were evaluated for the selected biomarkers. After the incubation of the biosensors with the selected aptamers, different concentrations of Troponin-T, CKMB and HER2 antigens, ranging from $0.1 \mathrm{pg} \mathrm{mL}^{-1}$ to $1 \mathrm{ng} \mathrm{mL} \mathrm{m}^{-1}$, were measured by EIS (cf. Figure 5.4).

(a)
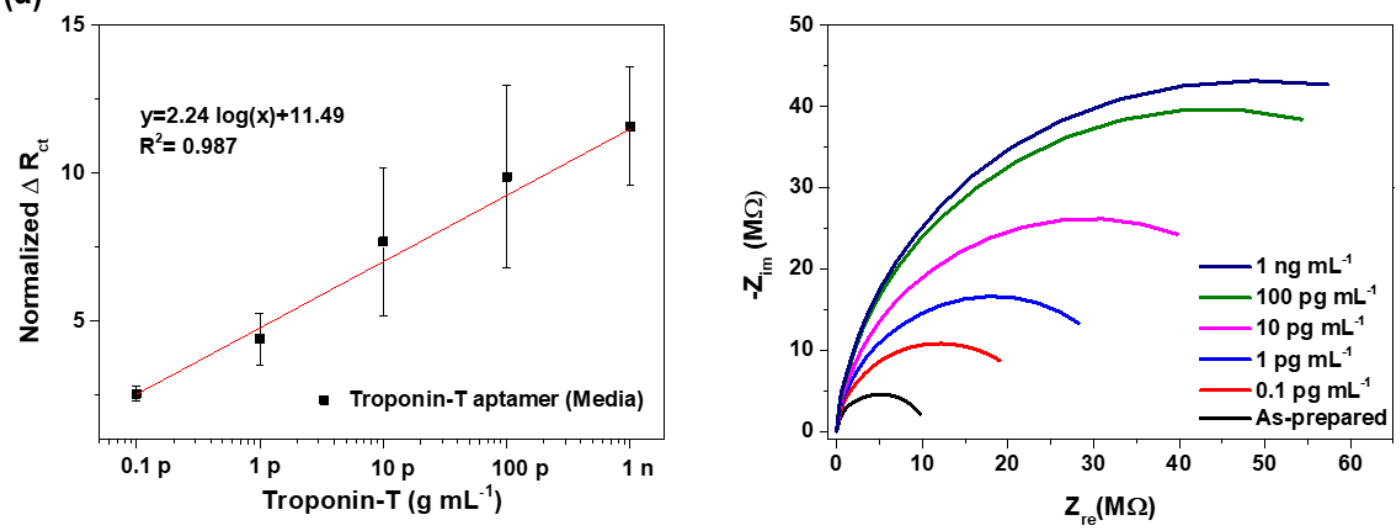

(b)
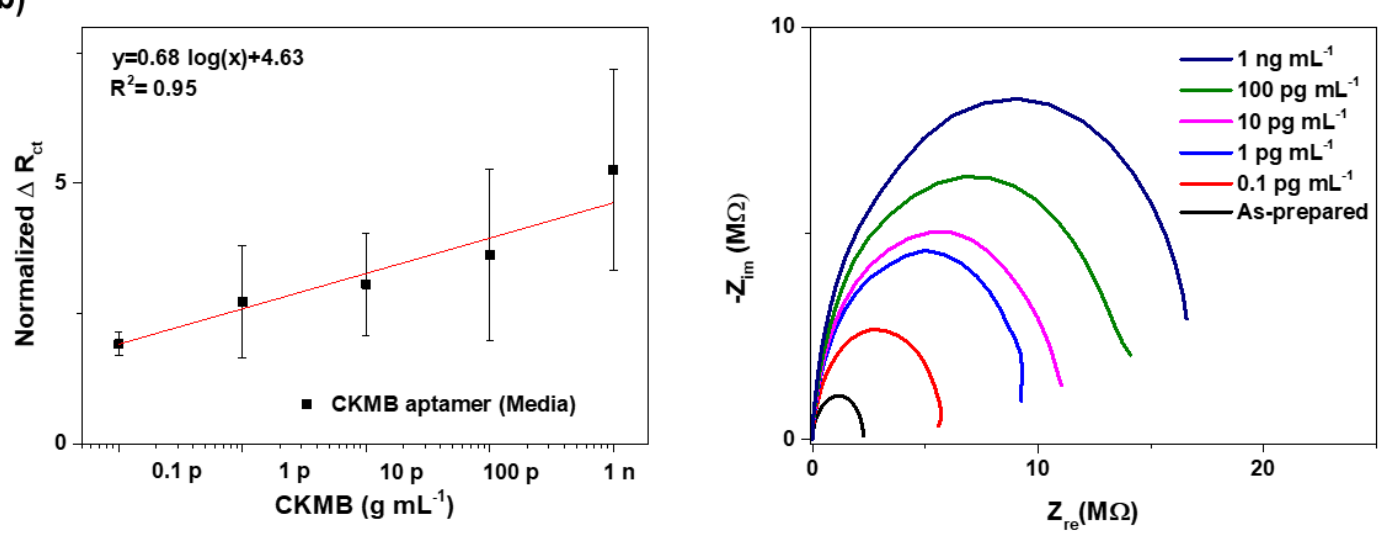

(c)
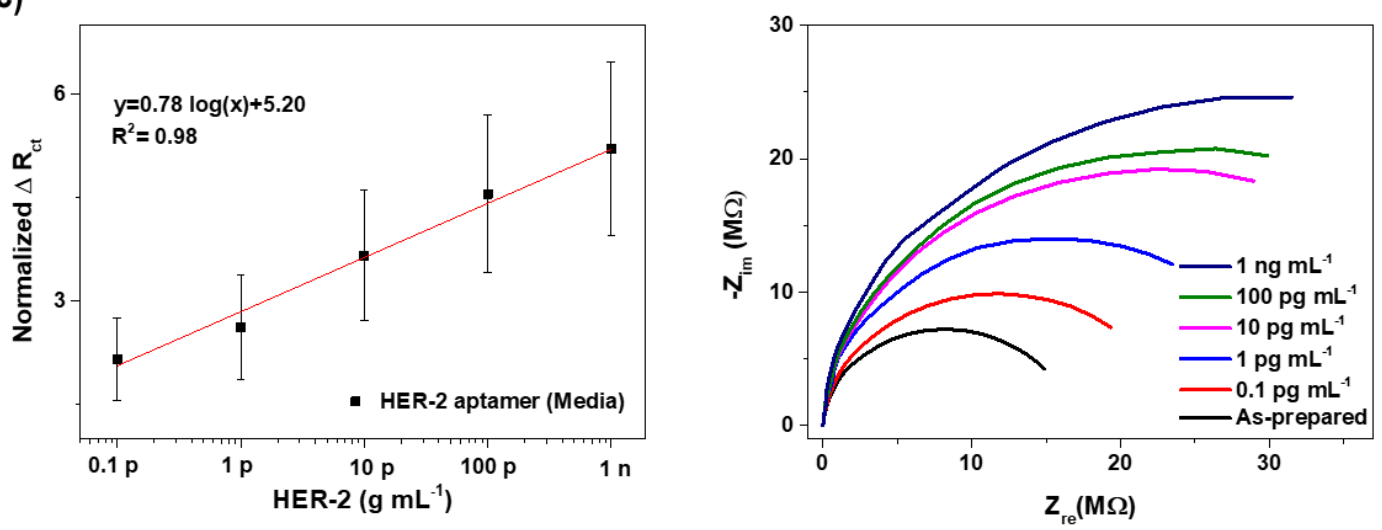

Figure 5.4. Calibration curves and respective Nyquist curves obtained at $37{ }^{\circ} \mathrm{C}$ for (a) cardiac Troponin-T aptamer; (b) human cardiac CKMB aptamer; and (c) breast cancer HER2 aptamer.

The successful functionalization of each aptamer layer on bare electrodes is confirmed by the subsequent increase of the impedance signal, as shown by the Nyquist curves in Figure 5.4. In a similar way, the successively attachment of increasing amounts of antigens is shown by the increase of the electron-transfer resistance $\left(R_{c t}\right)$ value obtained from each 
Nyquist curve, due to the redox probe $\left[\mathrm{Fe}(\mathrm{CN})_{6}\right]^{4-/ 3-}$ by the antigens. To obtain the calibration curves of the biomarkers and eliminate possible variations from the individual microelectrodes, $\Delta R_{c t}$ was normalized as $R_{c t}$ antigen/ $R_{c t}$ media, as reported above. In this study, $R_{c t}$ media is termed "as-prepared" signal and used as the baseline of the biosensors. The calibration curves were obtained by plotting the normalized $\Delta R_{c t}$ of the ranging concentration of the antigens $\left(0.1 \mathrm{pg} \mathrm{mL}^{-1}\right.$ to $\left.1 \mathrm{ng} \mathrm{mL}^{-1}\right)$ from the selected biomarkers.

The slope of the calibration curves for Troponin-T, CKMB and HER2 prepared in medium and measured by the aptamer-based biosensor were 2.24, 0.68 and $0.78\left(\log \left(\mathrm{pg} \mathrm{mL}^{-1}\right)\right)^{-1}$, respectively. These values show the high sensitivity of this aptamer-based biosensor to detect trace amounts of the selected biomarkers, even when they are dissolved in complex biological environments, such as cell culture medium. Overall, these results indicate that this aptamer-based biosensor possesses much higher sensitivity to detect trace amounts of biomarkers ( $\mathrm{pg} \mathrm{mL}^{-1}$ ) than other methods, including immunochemiluminometric assays (ng $\mathrm{mL}^{-1}$ ), amperometric assays ( $\mu \mathrm{g} \mathrm{mL}^{-1}$ ), or ELISA assays ( $\mathrm{ng} \mathrm{mL} \mathrm{L}^{-1}$ ) [9]. In this context, these aptamers-based biosensors show high potentiality to be used as an excellent biosensing platform to monitor with high sensitivity trace amount of several biomarkers produced by multi-organ-on-a-chip system during drug screening tests.

\subsubsection{In vitro 2D cellular studies of drug nanocarrier GYSMNP}

On a previous study using heart-on-a-chip systems [9], cardiomyocytes treated with $10 \mu \mathrm{M}$ of free DOX presented reduced beating rates, as well as reduced cellular viability. Thus, in this study, the concentration of $10 \mu \mathrm{M}$ of DOX was selected to perform the chemotherapeutic treatment of breast cancer organoids and to verify the effect of this drug on the healthy cardiac tissues.

As detailed in Section 4.3, graphene-based yolk-shell magnetic nanoparticles functionalized with copolymer PF-127 (GYSMNP@PF127) were successfully developed as multifunctional nanocarriers for the treatment of cancer. This nanosystem revealed an exceptional $91 \%$ of drug loading capacity of DOX $(1 / 1, \mathrm{w} / \mathrm{w})$ and controlled drug release triggered by the acidic tumour microenvironment (cf. Figure 4.10). In order to determine the optimal concentration of GYSMNP@PF127-DOX that allows the release of equal amount of free DOX $(10 \mu \mathrm{M})$ in $24 \mathrm{~h}$ at $37^{\circ} \mathrm{C}$, the breast cancer cell line $\mathrm{SkBr} 3$ was incubated with different concentrations of nanocarriers $\left(15,30\right.$ and $60 \mu \mathrm{g} \mathrm{mL}^{-1}$, corresponding to 10,20 and $40 \mu \mathrm{M}$ of total loaded DOX, respectively) and compared with those obtained with $10 \mu \mathrm{M}$ of free DOX (cf. Figure 5.5). After incubation, $\mathrm{SkBr} 3$ cells were stained with Calcein-AM, which is a membrane-permeable 
live-cell labelling dye that shows living cells in green. DOX images show the red intensity from the DOX uptake by the cells.
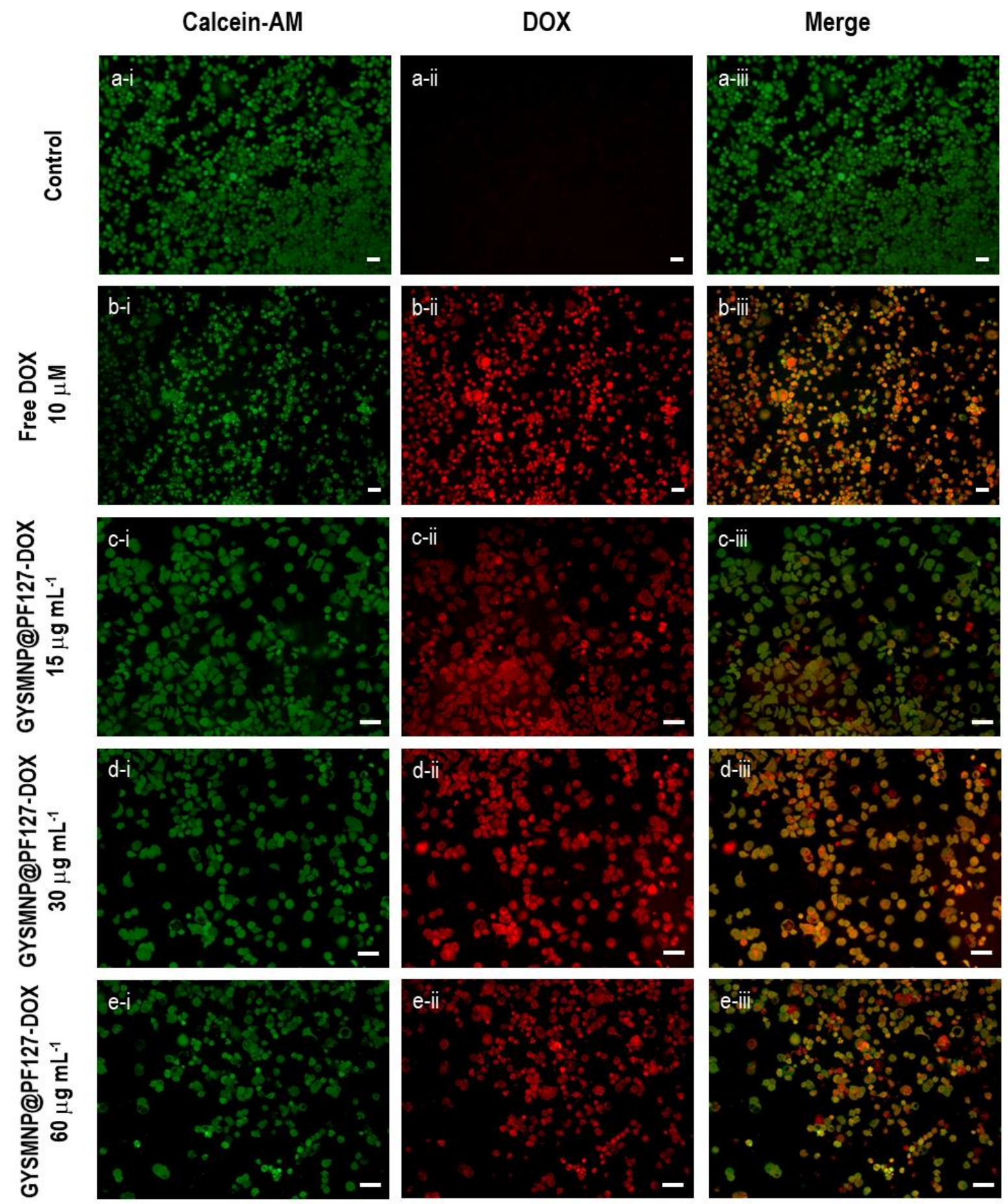

Figure 5.5. Confocal microscopy images of $\mathrm{SkBr} 3$ cells incubated during $24 \mathrm{~h}$ at $37^{\circ} \mathrm{C}$ : (a) without chemotherapeutic treatment; (b) free DOX $(10 \mu \mathrm{M})$; and (c-e) GYSMNP@PF127-DOX at different concentrations of 15,30 and $60 \mu \mathrm{g} \mathrm{mL}^{-1}$, corresponding to 10, 20, $40 \mu \mathrm{M}$ of loaded DOX, respectively. (i) cells stained with Calcein-AM excited at $495 \mathrm{~nm}$; (ii) DOX released in $\mathrm{SkBr} 3$ cells and excited at 561 $\mathrm{nm}$; (iii) cell merged images. Scale bars represent $100 \mu \mathrm{m}$. 
By observing $\mathrm{SkBr} 3$ cells treated with free DOX, it can be seen that the number of living cells are lower in comparison with control images, which means that cell viability was affected. In this context, the nanocarrier concentration that has the most similar effect when compared to free DOX, was achieved with $30 \mu \mathrm{g} \mathrm{mL}^{-1}$ (corresponding to $20 \mu \mathrm{M}$ of total loaded DOX). This result is in line with the in vitro drug release study shown in Figure 4.10, which shows that at $37{ }^{\circ} \mathrm{C}$ and acidic tumour microenvironments $(\mathrm{pH} 4.5)$, the drug nanocarrier GYSMNP@PF127 is able to release 50\% of the loaded DOX. Thus, the concentration of $30 \mu \mathrm{g}$ $\mathrm{mL}^{-1}$ of GYSMNP@PF127-DOX was selected to perform the chemotherapeutic treatment on the dual-organ-on-a-chip and compare its toxicological results on both organoids with free DOX $(10 \mu \mathrm{M})$.

Another important parameter to be considered is the biocompatibility and toxicity effect of the nanocarriers over the healthy and tumour cell lines. The cell biocompatibility of GYSMNP@PF127 (unloaded with DOX, $16.6 \mu \mathrm{g} \mathrm{mL} \mathrm{m}^{-1}$ ) and cytotoxicological effect of GYSMNP@PF127-DOX (loaded with DOX, $30 \mu \mathrm{gL}^{-1}$ ) were investigated by PrestoBlue colorimetric assay, as shown in Figure 5.6.

(a)

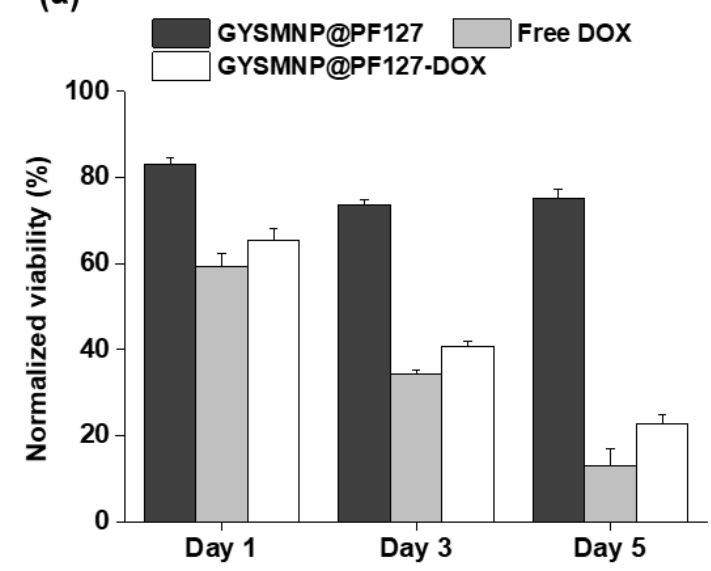

(b)

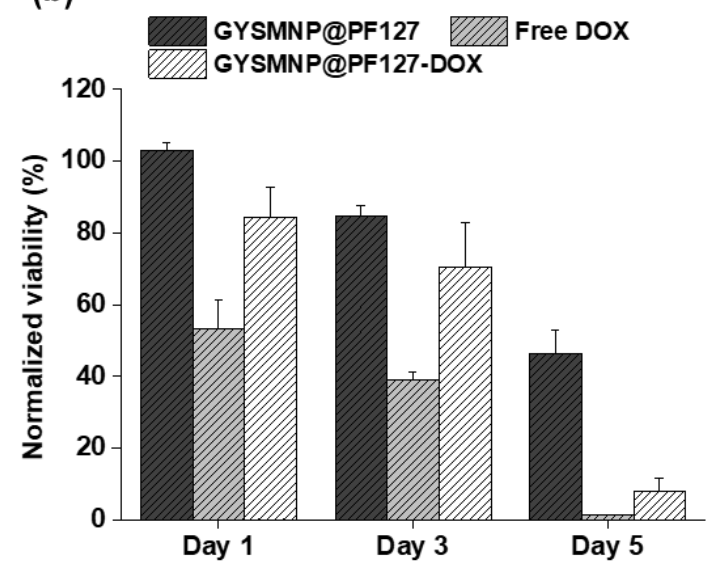

Figure 5.6. Cell cytotoxicity assays performed with GYSMNP@PF127, free DOX and GYSMNP@PF127DOX and investigated by PrestoBlue colorimetric assay during 5 days. (a) Breast cancer ( $\mathrm{SkBr} 3$ ); (b) healthy cardiomyocytes (iPSCs). Data are presented as mean \pm standard deviation of percentage viability compared with untreated cells $(n=4)$.

As observed in Figure 5.6, free DOX has high toxicological effect over both cells lines (breast cancer and healthy cardiomyocytes). In the first three days, unloaded GYSMNP@PF127 has no apparent toxicity over both cells lines. However, in the fifth day, cardiac cells show a decrease of viability compared to the previous days. One explanation for this drop of viability in day 5 is the cumulative effect of the magnetic nanoparticles that stay trapped in the GelMA structure along the sequential treatments. This factor increases the final concentration of nanomaterial into the hydrogel structure, thus increasing its toxicity to the 
cells. In any case, the overall results show that at least for three days of chemotherapeutic treatment, the developed nanocarriers are able to selectively deliver the anticancer drug in the tumour target and to protect the healthy cardiac tissue in comparison to free DOX. For a better understanding of this phenomenon, a dynamic microfluidic study using a dual-organon-a-chip was used to assess the effect of DOX (free and encapsulated) over both organs in continuous.

\subsubsection{Monitoring of cell-secreted biomarkers from bioreactor samples}

The dual-organ-on-a-chip platform was obtained by embedded cardiac iPSCs and $\mathrm{SkBr} 3$ spheroids inside a gelation methacryloyl (GelMA) hydrogel photo-cross linked, generating the human cardiac- and breast cancer-organ-on-a-chip bioreactor (Figure 5.7).

The development of the biomimetic dual-organ-on-a-chip system allows the simultaneous monitoring and investigation of the effect of chemotherapeutic drugs over aggressive breast cancer tumours (such $\mathrm{SkBr} 3$ cell line) and healthy cardiac tissues. This non-invasive continuous monitoring of multi-organ systems offers the possibility to obtain new insights over the cellular metabolic activity, as well as to develop new drug models or adjust chemotherapeutic doses in a personalized manner. To date, and to the best of our knowledge, this is the first study developed to determine the effect of anticancer drugs over multi-organs-on-a-chip platforms using biomolecular sensing.
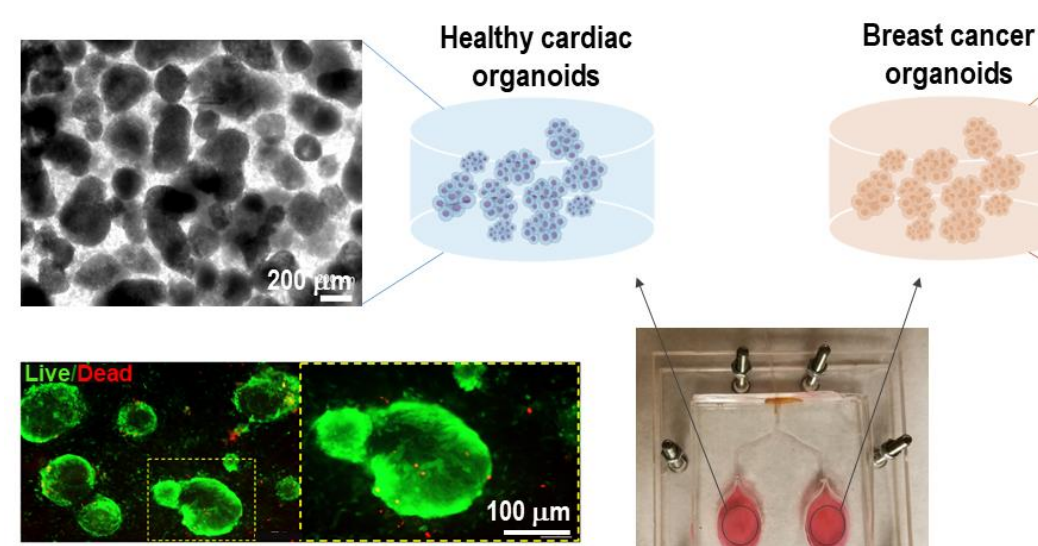

iPSCs spheroids
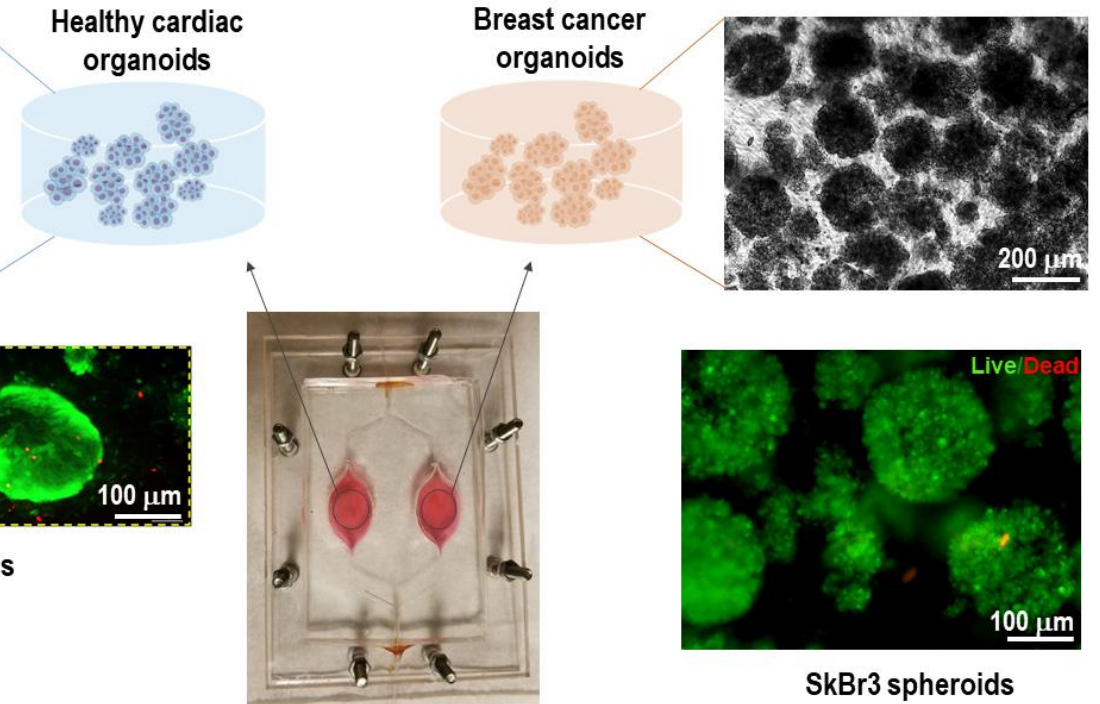

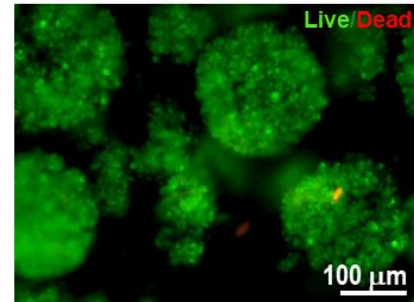

SkBr3 spheroids

Dual-organ-on-a-chip microfluidic bioreactor

Figure 5.7. Schematic representation of the dual-organ-on-a-chip microfluidic bioreactor developed to monitor the effect of the chemotherapeutic drug doxorubicin (free or encapsulated in nanocarriers) over the breast cancer and cardiac healthy organoids. Life/dead confocal images from iPSCs and SkBr3 spheroids showing the high viability of these organoids inside the GelMA hydrogel after UV photo-cross linking. Live cells stained green and dead cells stained red. 
To determine the effect of the anticancer drug DOX over the dual-organ-on-a-chip, two individual experiments were performed, as listed in Table 5.2.

Table 5.2. Anticancer drug induced experiments using dual-organ-on-a-chip microfluidic bioreactor

\begin{tabular}{cccc}
\hline Assay \# & Type & Organs & Drug test \\
\hline 1 & Dual-organ & SkBr3 + iPSCs & Free DOX \\
2 & Dual-organ & SkBr3 + iPSCs & GYSMNP@PF127-DOX \\
\hline
\end{tabular}

The exposure to DOX, the anticancer drug model used in this study and known to induce cardiac injury and heart failure [20], is expected to increase the levels of the cardiac biomarkers Troponin-T and CKMB, as well as to decrease the amount of HER2 biomarkers, due to the toxicological effect over the breast cancer tissues, as observed during the 2D cellular studies (Figure 5.6 (a)). The monitoring of each biomarker was followed during 5 days of successful culture of the organoids in the bioreactors without leakage or contamination. In selected days (1, 3 and 5), samples of co-culture media (StemPro + McCoy media, 1:1 (v/v)) were collected from the feedback loop system and analysed by electrochemical analysis using single microelectrodes (Figure 5.8 (a)).

(a)

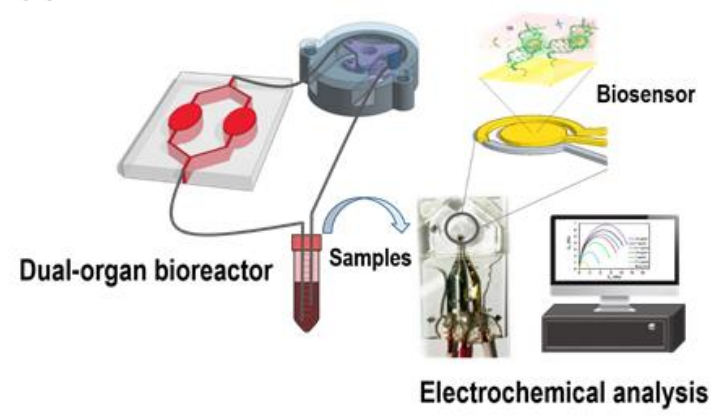

(c)

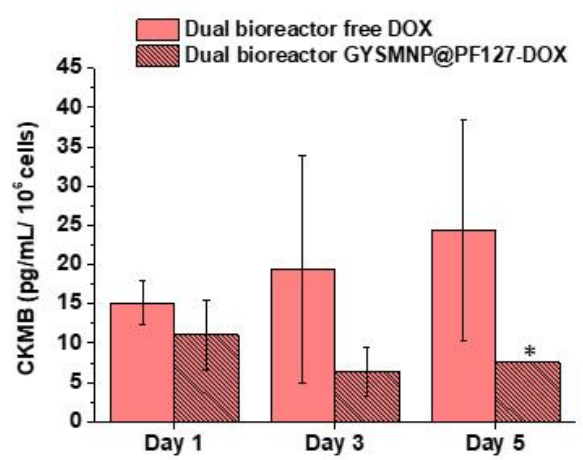

(b)

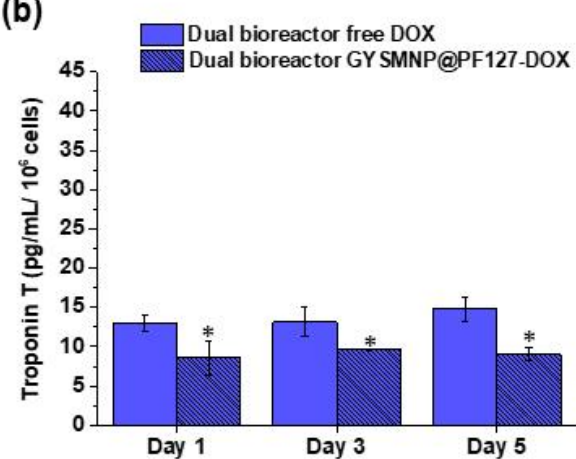

(d)

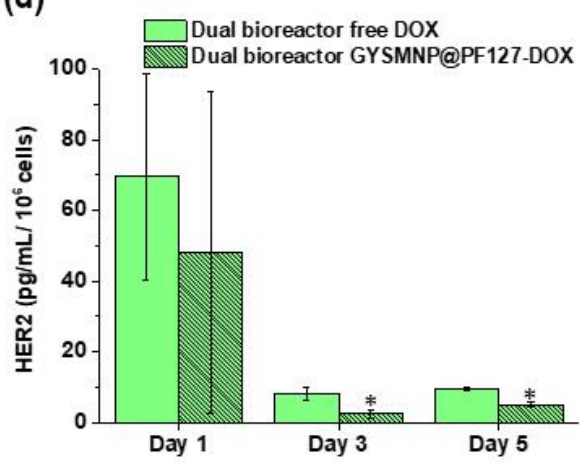

Figure 5.8. (a) Schematic representation of the feedback loop system used during the drug induced experiments on dual-organ-on-a-chip microfluidic bioreactor along 5 days. Electrochemical sensing of selected biomarkers released from dual organ-on-a-chip bioreactor: (b) cardiac biomarker Troponin$\mathrm{T}$; (c) cardiac biomarker CKMB; (d) breast cancer biomarker HER2. Error bars show standard deviation of individual single microsensors $(n=3)$ and the asterisks $\left(^{*}\right)$ represent statistical significance in comparison with free DOX assay for $p<0.05$ determined by Student's t-test. 
Samples collected from drug-induced experiments along 5 days were measured in individual aptamer-based biosensor for each biomarker by EIS (Figure 5.8 (b-d)). The results shown in Figure 5.8 reflect the normalized $\Delta R_{c t}$ data obtained from EIS measurements and correlated to each calibration curve (Figure 5.4). As expected, free DOX shows to induce a dramatic toxicity over the breast cancer tissue, as revealed by the decrease of HER2 biomarkers along the 5 days of chemotherapeutic treatment, but also reveals cardiac injury to the heart organoid with increasing detection of both cardiac diseases' biomarkers (i.e., Troponin-T and $(K M B)$. On the other hand, the encapsulated DOX released by the nanocarrier GYSMNP@PF127-DOX shows a more protective behaviour over the healthy cardiac organoids in comparison with the free DOX, and at the same time maintaining the severe toxicity over the breast cancer spheroids. These exciting results attest the previous screening results detailed in Section 4.3, where the developed graphene-based magnetic nanoparticles were tested as smart and on-demand drug nanocarriers triggered by the abnormal acidic $\mathrm{pH}$ found in tumour microenvironment.

In addition to the aptamer-based biosensing analysis, the cell viability of the cardiomyocytes and breast cancer organoids after the chemotherapeutic treatment (day 5) were analysed by live/DOX assay (cf. Figure 5.9).
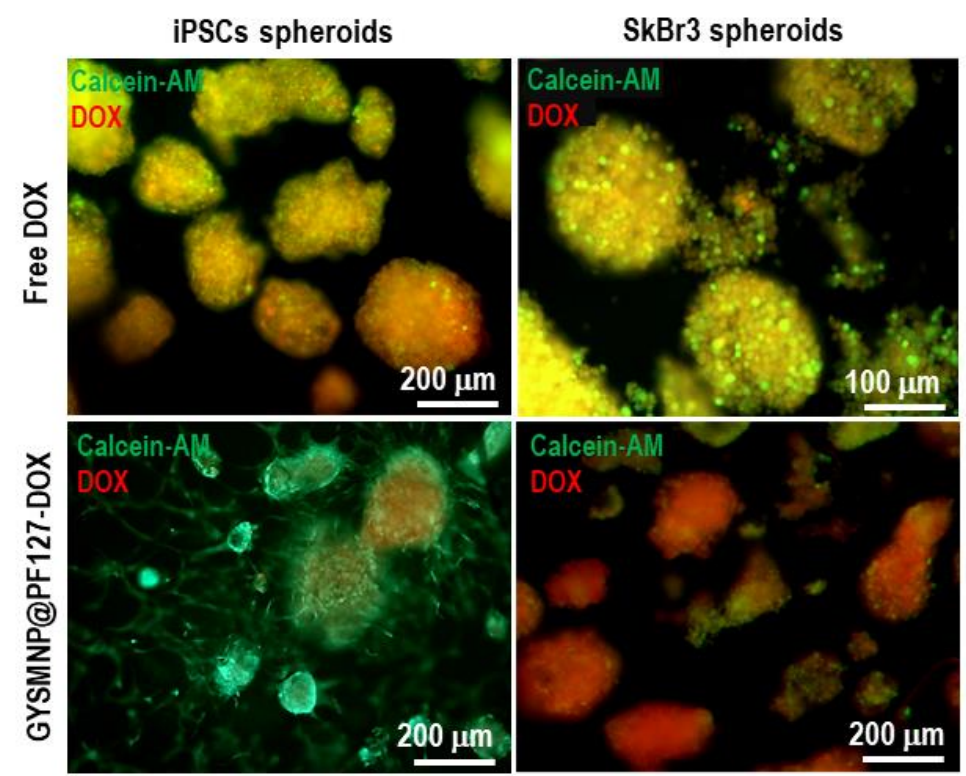

Figure 5.9. Cell viability assays of cardiomyocytes (iPSCs) and breast cancer ( $\mathrm{SkBr} 3$ ) spheroids obtained after 5 days of chemotherapeutic treatment (free or encapsulated DOX) in dual-organ-on-achip bioreactors. Live cells stained with green Calcein-AM dye and red cells showing DOX uptake.

Since DOX has the same fluorescence spectrum than that of red dye (that stain dead cells), the organoids were just stained with Calcein-AM dye (green dye for live cells). The red cells shown in Figure 5.9 reveals the DOX that was uptaked upon the chemotherapeutic treatment during 5 days. The confocal images subscribe the results obtained from the aptamer-based 
biosensing analysis, showing that free DOX is delivered in the same proportion on both organoids (healthy cardiac tissues and breast cancer), which cause the damage of cardiac tissues and the release of the cardiac biomarkers (Troponin-T and CKMB). On the other hand, DOX encapsulated in the graphene-based nanocarriers shows high-target delivery over the breast cancer organoids compared to cardiac spheroids, as ascribed by the low amount of cardiac biomarkers detected with the biosensor system. Once again, the results confirm the potentiality of the developed GYSMNP@PF127 material as smart and on-demand drug nanocarriers for the treatment of cancer.

These results also show the extraordinary ability to use the aptamer-based electrochemical biosensor to detect trace amounts ( $\mathrm{pg} \mathrm{mL}^{-1}$ ) of several biomarkers secreted from multi-organ systems, which were undetectable by using conventional methods, such as ELISA (ng $\mathrm{mL}^{-1}$ ). However, the presented study represents just the first steps to develop an integrated and automatized microfluidic platform comprising the dual-organ-on-a-chip system with EC multiplexed biosensor. This proposed platform (in final stage of development) will be able to detect and monitor up to 16 different biomarkers released from the dual-organ-on-a-chip, in continuous and for several days, during the drug screening tests (Figure 5.10).

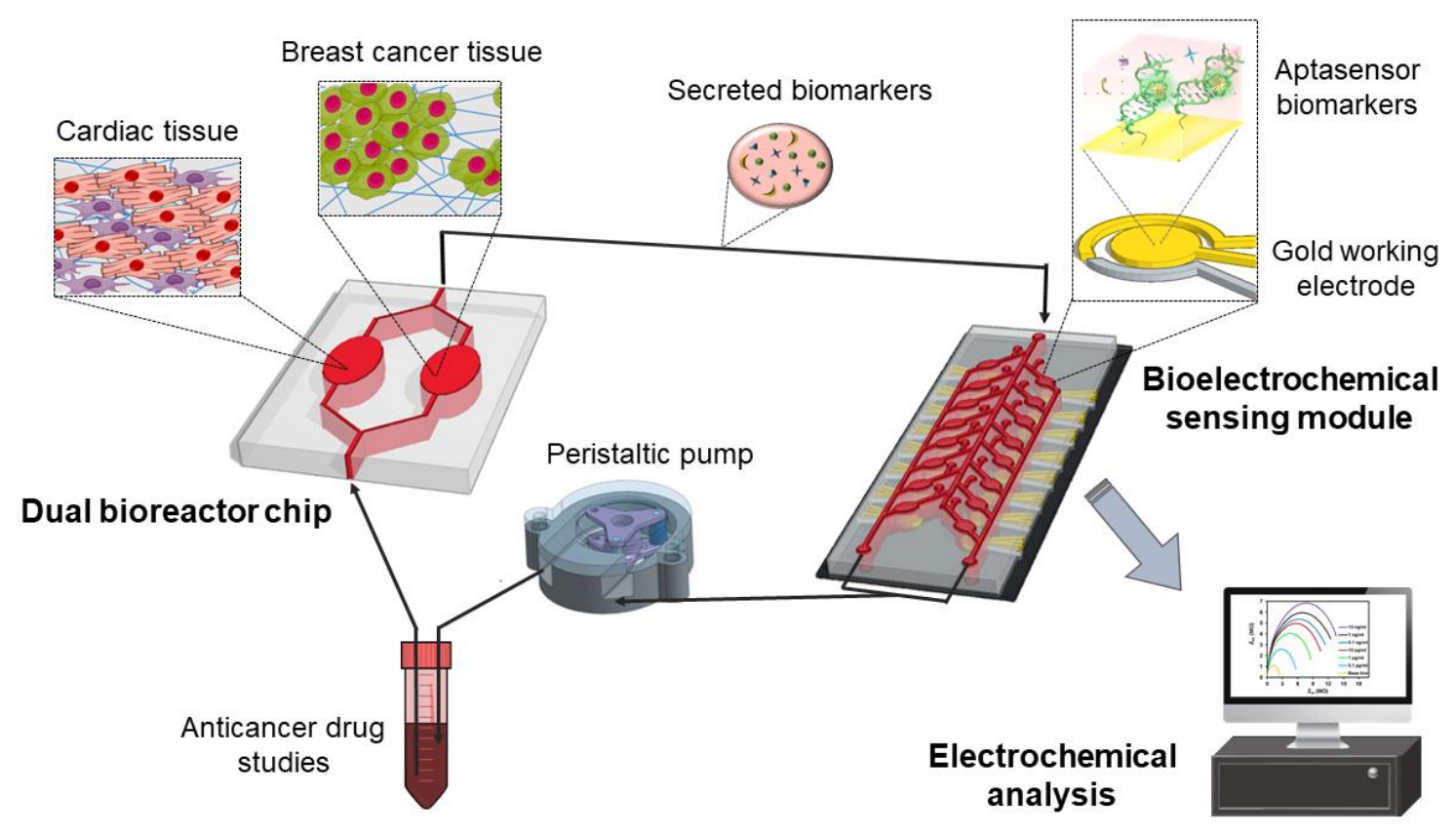

Figure 5.10. Schematic representation of the feedback loop of dual-organ platform (comprising breast cancer and cardiac organoids) integrated with multiplexed electrochemical analysis for the chemotherapy study effect of doxorubicin using the free or encapsulated drug.

The development of this platform based on aptamer-based microfluidic electrochemical biosensors is expected to find widespread applications to monitor multi-organ-on-a-chip platforms and give new insights in many biomedical scientific fields, including nanomedicine. 


\subsubsection{Conclusions}

Organ-on-a-chip platforms are suitable 3D tissue/organ models with the potentiality to better predict the physiological response of the human body to pharmacological compounds. Although chemotherapeutic drugs are a standard therapeutic treatment of cancer, the non-specificity of the majority of anticancer drugs make them the cause of secondary diseases during the chemotherapeutic treatment. This is the case of doxorubicin, an anticancer drug widely used in chemotherapy with significant cardiotoxicity. To overcome this drawback, nanomedicine is devoting efforts to develop smart drug nanocarriers that selectively deliver drug compounds in the tumours sites.

In this study, the results show the successful detection of trace amounts of cardiac biomarkers, such as Troponin-T and CKMB, as well as the breast cancer biomarker HER2. Those biosensing results ascribed the known side-effect of free DOX over the healthy cardiac tissue. Nevertheless, they also show that the chemotherapeutic treatment performed with graphene-based nanocarrier was able to protect the healthy cardiac tissue and selectively deliver the anticancer drug in the tumour organoid. These results were further confirmed by confocal imaging using life/DOX assays, which demonstrate the importance of performing dynamic microfluidic studies. The ultimate goal of this unique microfluidic electrochemical biosensor system is to be further integrated with multi-organ-on-a-chip platforms, creating in this way an integrated and automatized microfluidic platform comprising multi-organ-ona-chip system with electrochemical analysis. This multiplexed microfluidic platform is expected to find widespread applications in monitoring organ-on-a-chip platforms, and give significant insights in many biomedical scientific fields, including nanomedicine. 


\section{References}

[1] N. S. Bhise, J. Ribas, V. Manoharan, Y. S. Zhang, A. Polini, S. Massa, M. R. Dokmeci and A. Khademhosseini, Journal of Controlled Release, 2014, 190, 82-93.

[2] A. Khademhosseini and R. Langer, Nature Protocols, 2016, 11, 1775.

[3] L. A. Torre, R. L. Siegel, E. M. Ward and A. Jemal, Cancer Epidemiology Biomarkers Eamp; Prevention, 2016, 25, 16-27.

[4] T. L. A., B. Freddie, S. R. L., F. Jacques, L. T. Joannie and J. Ahmedin, CA: A Cancer Journal for Clinicians, 2015, 65, 87-108.

[5] R. T. Chlebowski, J. E. Manson, G. L. Anderson, J. A. Cauley, A. K. Aragaki, M. L. Stefanick, D. S. Lane, K. C. Johnson, J. Wactawski-Wende, C. Chen, L. Qi, S. Yasmeen, P. A. Newcomb and R. L. Prentice, JNCl: Journal of the National Cancer Institute, 2013, 105, 526-535.

[6] E. J. Benz, Transactions of the American Clinical and Climatological Association, 2017, 128, 275-297.

[7] P. Cinzia, G. S. Gabriele, M. Fabio, I. Federica, C. Giuseppe, G. Giuseppe, S. Federica, F. Marco, S. Fernando, C. Andreina, T. Bruno and E. Giovanni, Current Vascular Pharmacology, 2014, 12, 106-116.

[8] F. DeLuna, Y. S. Zhang, G. Bustamante, L. Li, M. Lauderdale, M. R. Dokmeci, A. Khademhosseini and J. Y. Ye, in SPIE BiOS, SPIE, 2018, p. 6.

[9] S. R. Shin, Y. S. Zhang, D.-J. Kim, A. Manbohi, H. Avci, A. Silvestri, J. Aleman, N. Hu, T. Kilic, W. Keung, M. Righi, P. Assawes, H. A. Alhadrami, R. A. Li, M. R. Dokmeci and A. Khademhosseini, Analytical Chemistry, 2016, 88, 10019-10027.

[10] C. L. Waite and C. M. Roth, Critical reviews in biomedical engineering, 2012, 40, 2141.

[11] H.-G. Yi, H. Lee and D.-W. Cho, Bioengineering, 2017, 4, 10.

[12] Y. S. Zhang, J. Aleman, S. R. Shin, T. Kilic, D. Kim, S. A. Mousavi Shaegh, S. Massa, R. Riahi, S. Chae, N. Hu, H. Avci, W. Zhang, A. Silvestri, A. Sanati Nezhad, A. Manbohi, F. De Ferrari, A. Polini, G. Calzone, N. Shaikh, P. Alerasool, E. Budina, J. Kang, N. Bhise, J. Ribas, A. Pourmand, A. Skardal, T. Shupe, C. E. Bishop, M. R. Dokmeci, A. Atala and A. Khademhosseini, Proceedings of the National Academy of Sciences, 2017, 114, E2293-E2302.

[13] Y. S. Zhang, Y.-N. Zhang and W. Zhang, Drug Discovery Today, 2017, 22, 1392-1399.

[14] S. R. Shin, T. Kilic, Y. S. Zhang, H. Avci, N. Hu, D. Kim, C. Branco, J. Aleman, S. Massa, A. Silvestri, J. Kang, A. Desalvo, M. A. Hussaini, S. K. Chae, A. Polini, N. Bhise, M. A. Hussain, H. Lee, M. R. Dokmeci and A. Khademhosseini, Advanced Science, 2017, 4, 1600522.

[15] R. E. Gerszten and T. J. Wang, Nature, 2008, 451, 949.

[16] A. V. Lakhin, V. Z. Tarantul and L. V. Gening, Acta Naturae, 2013, 5, 34-43.

[17] P. R. Puleo, D. Meyer, C. Wathen, C. B. Tawa, S. Wheeler, R. J. Hamburg, N. Ali, S. D. Obermueller, F. J. Triana and J. L. Zimmerman, New England Journal of Medicine, 1994, 331, 561-566.

[18] N. Iqbal and N. Iqbal, Molecular Biology International, 2014, 2014, 852748.

[19] T. Fehm, S. Becker, S. Duerr-Stoerzer, K. Sotlar, V. Mueller, D. Wallwiener, N. Lane, E. Solomayer and J. Uhr, Breast cancer research : BCR, 2007, 9, R74-R74. 
[20] G. Minotti, P. Menna, E. Salvatorelli, G. Cairo and L. Gianni, Pharmacological Reviews, 2004, 56, 185. 

CHAPTER VI

Conclusions and future perspectives 



\section{Conclusions and future perspectives}

The main objectives of this work are the development of carbon-based magnetic nanoparticles suitable for nanomedicine and their microfluidic study using new bioplatforms, aiming new insights over the complex biophysical and toxicological interaction of these nanosystems with human cells (i.e. blood, healthy and tumour cells). The successful achievement of these objectives is involved in complex multidisciplinary approaches, namely chemistry, physics, materials science, biology, bioengineering and biomechanics, which were accomplished by subdividing these objectives in subsequent tasks and chapters.

In the first task, described in Chapter III, superparamagnetic nanoparticles were synthesized, characterized and tested for theranostic applications (i.e., magnetic hyperthermia and MRI). Aiming new insights on the effect of these nanomaterials over the human body, a new microfluidic methodology for the haemocompatibility assessment was developed. This highsensitivity technique is expected to bring a better understanding of the biological impact of nanoparticles developed for clinical applications when applied directly into the bloodstream. Additionally, in this chapter, a thermal infrared image processing was considered to assess the heat generated by magnetic nanoparticles during hyperthermia. This imaging processing methodology can be used as a screening tool to evaluate the potentiality of MNPs synthesized for hyperthermia when a home-made AC magnetic field apparatus is used.

In Chapter IV, a tailor-made protocol capable to synthesize graphene-based yolk-shell magnetic nanoparticles (GYSMNPs) for nanomedicine was developed. Particularly, a set of GYSMNP revealed outstanding features to be applied as dual exogenous (AC field)/endogenous $(\mathrm{pH})$ stimuli-responsive actuators for targeted thermo-chemotherapy, combining magnetic hyperthermia and controlled drug release triggered by the abnormal tumour environment.

Aiming new insights over this promising multifunctional magnetic carbon nanostructures, labelled as GYSMNP@PF127, Chapter V details a novel dual-organ-on-a-chip platform combined with electrochemical biosensing for drug screening tests. The electrochemical analysis from this 3D microfluidic tissue/organ model, allied to conventional 2D cellular studies, ascribed the potentiality of the developed graphene-based nanosystems to be used as smart drug delivery nanocarriers triggered by tumour microenvironments. The development of this platform based on aptamer-based microfluidic electrochemical 
biosensors is expected to find widespread application to monitor multi-organ-on-a-chip platforms and give new insights in many biomedical scientific fields, including nanomedicine.

Overall, the developed carbon-based nanosystems, as well as the proposed microfluidic platforms described in this work, allows to contribute to a better understanding of the biophysical and biological interaction of new biomedical nanoplatforms designed for nanomedicine.

As future work, novel and advanced microfluidic devices comprising tissues/organs models with microenvironment biophysical and biochemical sensors (such as $\mathrm{pH}$, temperature, $\mathrm{O}_{2}$ and $\mathrm{CO}_{2}$ levels) are desirable to obtain multitude sensing data to better characterize and screen nanoparticles developed for biomedical applications. On the other hand, the multifunctional potentiality of the developed GbMNP for theranostic applications, i.e. as contrast enhancement in MRI to be combined with thermo-chemotherapy, can be further evaluated. Moreover, these advanced nanomaterials also show great potentiality to be functionalized with specific antibodies to enhance tumour targeting, or even with DNA/RNA for gene therapy. In this sense, the evolution of smart and multifunctional nanomaterials in nanomedicine could be very beneficiated by the parallel evolution of organ-on-a-chip platforms as 3D model systems to study their safety, bio-distribution and efficacy.

Thus, it is my perspective that the understanding of such complex biophysical interactions has to be achieved with the force of multidisciplinary groups, working in close contact to fill the gaps between nanomedicine and clinical applications. On this demand, organ-on-a-chip platforms can have an important impact in the field. There are still many challenges to be addressed on the development and optimization of these advanced 3D microfluidic platforms, but the potential and possibilities are far more enthusiastic. Therefore, and as predicted by Professor Langer and Khademhosseini, pioneers in drug delivery and tissue engineering [1]: "with sufficient time and research the promise of nanotechnology based medicine may become a reality".

\section{References}

[1] A. Khademhosseini and R. Langer, Chemical Engineering Progress, 2006, 102, 38-42. 


\section{Appendixes}

A. Deformation of Red Blood Cells, Air Bubbles, and Droplets in Microfluidic Devices: Flow Visualizations and Measurements

B. A simple microfluidic device for the deformability assessment of blood cells in a continuous flow

C. Polymer microfluidic devices: an overview of fabrication methods

D. Publications

E. Communications in scientific meetings

F. Grants and awards 



\title{
A. Deformation of Red Blood Cells, Air Bubbles, and Droplets in
}

\section{Microfluidic Devices: Flow Visualizations and Measurements}

Review

\section{Deformation of Red Blood Cells, Air Bubbles, and Droplets in Microfluidic Devices: Flow Visualizations and Measurements}

\author{
David Bento ${ }^{1,2,+}$, Raquel O. Rodrigues ${ }^{1,3,+}$, Vera Faustino ${ }^{4,+}$, Diana Pinho ${ }^{1,2,5,+}$ (c), \\ Carla S. Fernandes ${ }^{1}$, Ana I. Pereira ${ }^{1,5,6}$, Valdemar Garcia ${ }^{1}$, João M. Miranda ${ }^{2, *}$ \\ and Rui Lima $2,7, *$ (C) \\ 1 Instituto Politécnico de Bragança, ESTiG/IPB, C. Sta. Apolónia, 5301-857 Bragança, Portugal; \\ davidbento@ipb.pt (D.B.); raquel.rodrigues@ipb.pt (R.O.R.); diana@ipb.pt (D.P.); cveiga@ipb.pt (C.S.F.); \\ apereira@ipb.pt (A.I.P.); valdemar@ipb.pt (V.G.) \\ 2 CEFT, Faculdade de Engenharia da Universidade do Porto (FEUP) Rua Roberto Frias, \\ 4800-058 Porto, Portugal \\ 3 LCM - Laboratory of Catalysis and Materials-Associate Laboratory LSRE/LCM, Faculdade de Engenharia \\ da Universidade do Porto (FEUP) Rua Roberto Frias, 4800-058 Porto, Portugal \\ 4 MEMS-UMinho Research Unit, Universidade do Minho, DEI, Campus de Azurém, \\ 4800-058 Guimarães, Portugal; vera_f_87@hotmail.com \\ 5 Centro de Investigação em Digitalização e Robótica Inteligente (CeDRI), Instituto Politécnico de Bragança, \\ Campus de Santa Apolónia, 5300-253 Bragança, Portugal \\ 6 Algoritmi R\&D Centre, Campus de Gualtar, University of Minho, 4710-057 Braga, Portugal \\ 7 MEtRiCS, Mechanical Engineering Department, Campus de Azurém, University of Minho, \\ 4800-058 Guimarães, Portugal \\ * Correspondence: jmiranda@gmail.com (J.M.M.); rl@dem.uminho.pt (R.L.); \\ Tel.: +351-22-508-1400 (J.M.M.); +351-25-351-0233 (R.L.) \\ + These authors contributed equally to this work.
}

Received: 10 February 2018; Accepted: 21 March 2018; Published: 27 March 2018

\begin{abstract}
Techniques, such as micropipette aspiration and optical tweezers, are widely used to measure cell mechanical properties, but are generally labor-intensive and time-consuming, typically involving a difficult process of manipulation. In the past two decades, a large number of microfluidic devices have been developed due to the advantages they offer over other techniques, including transparency for direct optical access, lower cost, reduced space and labor, precise control, and easy manipulation of a small volume of blood samples. This review presents recent advances in the development of microfluidic devices to evaluate the mechanical response of individual red blood cells (RBCs) and microbubbles flowing in constriction microchannels. Visualizations and measurements of the deformation of RBCs flowing through hyperbolic, smooth, and sudden-contraction microchannels were evaluated and compared. In particular, we show the potential of using hyperbolic-shaped microchannels to precisely control and assess small changes in RBC deformability in both physiological and pathological situations. Moreover, deformations of air microbubbles and droplets flowing through a microfluidic constriction were also compared with RBCs deformability.
\end{abstract}

Keywords: red blood cells; deformation index; microfluidic devices; air bubbles; droplets; blood flow

\section{Introduction}

Blood flow behavior in microcirculation is strongly influenced by red blood cell (RBC) deformability as they occupy almost half of whole blood volume. When RBCs are subjected to large external flow forces, they elongate without rupture and tend to return to their original shape when the external 
forces are removed. Some major determinants of RBC deformability include external flow forces, cell geometry, cell internal viscosity, and membrane viscoelastic properties [1]. RBC-related diseases, such as malaria, sickle cell disease, and diabetes, can also promote significant alteration in the RBC deformability. Ever since the deformability of RBCs became a potential biomarker for several blood diseases, various experimental techniques have been developed to measure the deformation of blood cells (see Table 1). There have been several reviews discussing different techniques for measuring RBC deformability under a variety of experimental and diseased conditions [1-4]. The recent progress in microfabrication and high-speed microvisualization technology made it possible to produce microfluidic devices able to directly visualize and characterize the mechanical properties of individual cells flowing through constriction microchannels $[5,6]$. However, there are still few reviews focusing on the use of these kinds of microfluidic devices to measure cell deformability. Most of the recent reviews, performed by Zheng et al. [7], Tomaiuolo [8], and Xue et al. [9], have focused on single-cell devices, cylindrical glass capillaries, and in microdevices, where the shear effect is dominant. Due to the growing interest of combining the shear and extensional effect to perform deformability measurements, this review focuses on the most recent findings performed by our research group related to the deformation of RBCs flowing through hyperbolic, smooth, and sudden-contraction microchannels. Moreover, deformations of air microbubbles flowing within in vitro blood microfluidic devices are also measured and compared with RBC deformability.

Table 1. Techniques to measure RBC deformability under different diseased conditions.

\begin{tabular}{|c|c|c|c|}
\hline Measurement Technique & Human Diseases & Main Key Features & References \\
\hline Micropipette aspiration & Sickle cell anemia, malaria & $\begin{array}{l}\text { Enables accurate mechanical response of } \\
\text { single RBCs, labor-intensive, } \\
\text { time-consuming, and involves a typically } \\
\text { difficult process of manipulation. }\end{array}$ & {$[4,10-12]$} \\
\hline Optical tweezers & $\begin{array}{l}\text { Malaria, sickle cell anemia, } \\
\text { diabetes mellitus }\end{array}$ & $\begin{array}{l}\text { Ability to obtain a mechanical response of } \\
\text { single RBCs down to the piconewton level; } \\
\text { labor-intensive, time-consuming and special } \\
\text { human technical skills are required. }\end{array}$ & {$[4,13,14]$} \\
\hline Atomic force microscopy & $\begin{array}{l}\text { Cancer, spherocytosis, } \\
\text { thalassemia, diabetes mellitus, } \\
\text { sickle cell anemia }\end{array}$ & $\begin{array}{l}\text { Ability to apply forces to RBC surfaces at the } \\
\text { nanoscale level; labor-intensive; } \\
\text { time-consuming, and requires } \\
\text { expensive equipment. }\end{array}$ & {$[4,15-17]$} \\
\hline Microfluidic ektacytometer & Diabetes mellitus & $\begin{array}{l}\text { Homogenous flow, ability to differentiate } \\
\text { healthy and diseased cells, labor-intensive } \\
\text { and time-consuming process. It is required to } \\
\text { label the RBCs to identify them. This latter } \\
\text { process may change the RBCs' } \\
\text { mechanical properties. }\end{array}$ & {$[18,19]$} \\
\hline Microfluidic constriction channel & $\begin{array}{l}\text { Diabetes mellitus, malaria, } \\
\text { cancer, abdominal obesity and } \\
\text { metabolic syndrome }\end{array}$ & $\begin{array}{l}\text { Reduced space, homogenous flow, label-free, } \\
\text { ability to measure a large amount of cells in } \\
\text { one single run, potential to precisely control } \\
\text { and detect small deformability changes, } \\
\text { needs a high-speed video microscopy system } \\
\text { combined with an image analysis technique; } \\
\text { blockage is likely to happen at constriction } \\
\text { microchannels with dimensions similar to the } \\
\text { RBC diameter. }\end{array}$ & [18-24] \\
\hline
\end{tabular}

\section{Deformation of RBCs in Microfluidic Devices}

Most of the proposed microfluidic devices to perform RBC deformability characterization focus on the shear effect. Some examples from the literature are the measurements of the RBCs' deformation under a transient high shear stress in a sudden-contraction microchannel [25] and the RBCs' deformability through a microfluidic device with a microchannel diameter comparable to RBC size [26]. In addition to the shear effect, the extensional effect and the combination of both can be encountered in the human body, e.g., in microstenosis, in microvascular networks composed of small, irregular vessel segments, in pulmonary microvessels, and in medical instrumentation, such as the 
flow through syringes and syringe needles. Hence, it is important to understand the RBC mechanical properties under both shear and extensional effect.

Flows of blood cells through microfluidic contractions generate complex flow phenomena despite their simple geometry. The flow involves a reduction in the cross-sectional area, which generates strongly-converging flows as the fluid goes through the contraction and the blood cells exhibit a variety of shapes, such as circular, ellipse, and parachute, which depend on the rheological properties of the fluid, geometric configuration, and dimensions of the contraction. The schematic illustration in Figure 1 shows the fluid flow behavior in different kinds of microfluidic constriction channels through which the RBCs travel. In general, the flow exhibits mixed kinematics with strong extensional flow (the fluid accelerates as it is going through the contraction) along the centerline and shear flow close to the walls. The major advantage of microfluidic hyperbolic-shaped contraction (Figure 1c) is the ability to impose a constant strain rate along the centerline of the contraction, as well as to achieve high extensional and shear flows. Relevant works in the context of blood flow and RBC deformability are those performed by Sousa et al. [27], Lee at al. [28], Yaginuma et al. [29], Rodrigues et al. [30-32], Faustino et al. [33], Pinho et al. [19], and Calejo et al. [34], who studied the effects of the extensional flow in hyperbolic converging microchannels using blood analog fluids and in vitro blood containing different kinds of blood cells. Other works have used cross-slot microfluidic devices to investigate the deformability of different kinds of cells under the application of extensional flows [35-37].

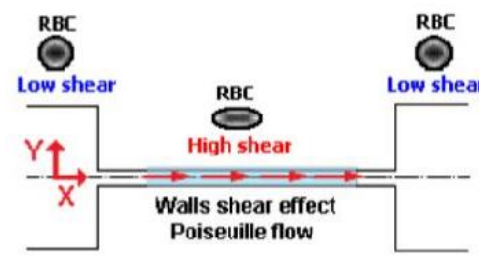

Sudden Contraction a)

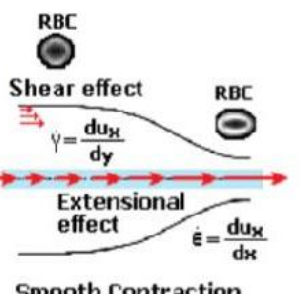

b)

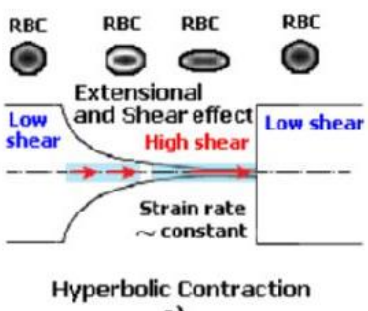

c)

Figure 1. Blood flow and RBC deformability in microfluidic contractions at different geometries:

(a) sudden contraction; (b) smooth contraction; and (c) hyperbolic contraction, adapted from [38].

RBCs flowing through microfluidic contractions are, most of the time, subjected to high shear and extensional effects and, as a result, they tend to elongate into ellipsoid shapes with their major axis aligned to the flow direction. When the cells leave the constriction region, the fluid shear forces created by the wall are removed and, consequently, RBCs tend to return to their normal resting biconcave disc shape. The deformation under controlled flow conditions provides an efficient method to generate cellular-scale mechanical stimuli. Hence, microfluidic constrictions due to the ability of performing precise control and manipulation of a small volume of samples have been gaining increasing interest to measure the deformability of RBCs for clinical proposes [5,7,8,19,28-33].

The classical method to quantify the degree of deformability is by using an ellipse-fitting program. The deformation index (DI), also known as elongation index, most of the times is calculated by $(\mathrm{X}-\mathrm{Y}) /(\mathrm{X}+\mathrm{Y})$ where $\mathrm{X}$ and $\mathrm{Y}$ represent the major and minor lengths of the ellipse, respectively (see Figure 2). However, in microchannel capillaries, where the dimensions of the channels are smaller than the diameters of the cells, the degree of deformability should be measured as the ratio between the length of the major axis and the length of the minor axis as the RBC tends to deform in a parachute shape, as shown in Figure 2 [20,39]. This latter definition is designated as the deformation ratio (DR). 


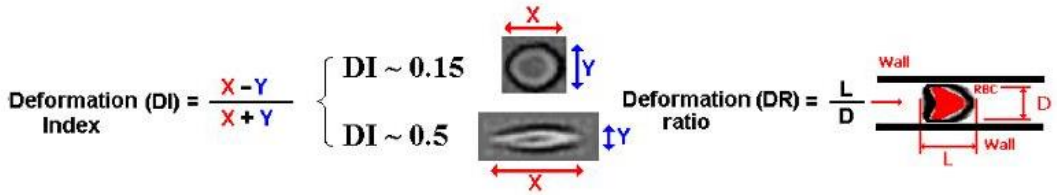

Figure 2. Schematic diagram of the deformation index (DI) and deformation ratio (DR) definition, adapted from [17].

\subsection{Deformation of RBCs in Hyperbolic Contractions}

Since RBC deformability became a potential clinical biomarker, several single-cell microfluidic methodologies have been developed to perform flow measurements on RBCs [6-9]. The majority of the methods to measure the RBCs' deformability have focused on the response of the cells under simple shear flow. However, it is well known that extensional flow also plays an important role in the blood cells' flow dynamics in both in vivo and in vitro environments. Extensional effects, or a combination of shear and extensional effects, can happen in several situations, such as in micro-contractions (due to velocity transition), in bifurcations (around the apex region and small branch), and when cells flow from a wide blood vessel to a narrow catheter or needles. This latter situation can generate extremely high extensional flows, which can promote hemolysis and, as a result, can lead to clogging and jamming within the devices $[28,35]$. Hence, recently, several extensional flow studies have been performed not only to assess cell deformability [19,28,29,31-33], but also to separate blood cells from plasma $[29,30]$. The majority of these studies were performed at hyperbolic converging microchannels where single-cell deformability was assessed under a controlled homogeneous extensional flow field. Figure 3 shows RBCs flowing through the expansion region (A) and hyperbolic contraction region (B) for two different flow rates, i.e., $9.45 \mu \mathrm{L} / \mathrm{min}$ and $66.15 \mu \mathrm{L} / \mathrm{min}$. These qualitative flow visualizations clearly show that the RBC deformability is higher in the hyperbolic contraction region (B) where the RBCs are subjected to a strong extensional flow. Right after the exit of the contraction, RBCs tend to recover their initial shape (A), which corresponds to a minimal value of the deformation and where the RBCs are no longer exposed to a strong extensional flow. Another expected result is that the RBCs have a tendency to increase the deformation as the flow rate increases. More detailed information can be found in the work performed by Yagimuma et al. [29] where they have investigated the influence of the extensional flow on the motion and deformability of individual RBCs in the full length of a hyperbolic microchannel.

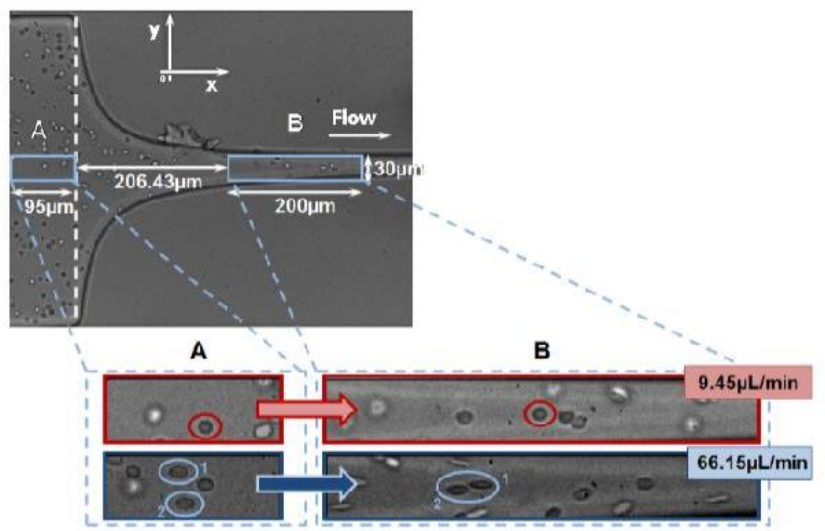

Figure 3. RBC deformability in a hyperbolic converging microchannel at two different regions (A) and (B) and flow rates $(9.45 \mu \mathrm{L} / \mathrm{min}$ and $66.15 \mu \mathrm{L} / \mathrm{min}$ ) (adapted from [40]). 
Figure 4 shows a quantitative description of the degree of deformation of human RBCs under a homogenous extensional flow field. We have measured the DI, as well as the velocity of the RBCs flowing through the expansion and hyperbolic contraction regions, for two different flow rates. For an inlet flow rate of $9.45 \mu \mathrm{L} / \mathrm{min}$ the RBCs do not suffer any significant deformation and the DI values are fairly constant along the full length of the microchannel. However, for a flow rate of $66.15 \mu \mathrm{L} / \mathrm{min}$ it is clear that when the RBCs enter the contraction region, RBCs start to elongate and, consequently, their DI values start to increase until the end of the hyperbolic contraction region. The latter results clearly show that when the RBCs are subjected to strong extensional flows RBCs tend to elongate up to a maximum value. Another interesting result shown in Figure $4 \mathrm{~b}$ is that when RBCs reach the hyperbolic contraction region their velocities increase almost linearly, which corresponds to a constant strain rate. This phenomenon happens for the both tested flow rates. These in vitro blood experiments show the potential of using hyperbolic-shaped microchannels to precisely control and assess changes in RBC deformability in physiological and pathological situations. However, the selection of the geometry and the identification of the most suitable region to evaluate the changes on the RBC deformability under strong extensional flows are crucial and further studies need to be performed in more detail in the near future.

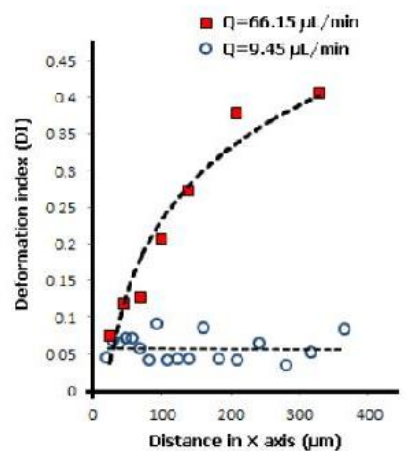

a)

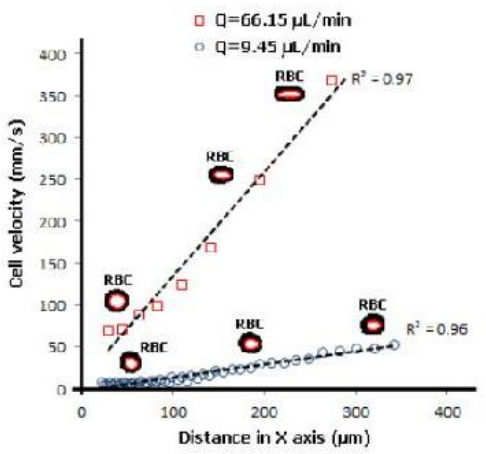

b)

Figure 4. Individual RBCs' (a) DI and (b) velocity flowing through a hyperbolic contraction microchannel for two different flow rates: $9.45 \mu \mathrm{L} / \mathrm{min}$ and $66.15 \mu \mathrm{L} / \mathrm{min}$.

Lee and his collaborators [28] have compared the deformability response of the RBCs to simple shear and extensional flows. Their results have shown that extensional flows generate higher RBC deformability than simple shear flows. Recently, Faustino and her colleagues $[33,38]$ have performed RBC deformation measurements in a hyperbolic-shaped contraction with a low aspect ratio (AR), where RBCs were submitted to both extensional and shear flow. By comparing the DI results performed by Faustino et al. $[33,38]$ with the results obtained with extensional flows $[29,40]$ it is clear that the combination of both extensional and shear flow promote higher RBC deformability. Although hyperbolic converging microchannels with low AR show the most suitable approach to assess the clinical meaning of RBC deformability, further studies should be performed with different flow rates and microchannel dimensions.

\subsection{Deformability in Smooth and Sudden Contractions}

During the years, a large number of studies on in vitro blood rheology and particularly in the deformation of RBCs under a simple shear flow were performed by using rotational rheometers [4,19,41-43]. However, RBCs flowing in microvessels, due to the confined microenvironment, deform not only due to shear effect but also to extensional effect. Hence, from the beginning of the 21st century, and due to the progress in microfabrication $[6,7,44,45]$, microflow visualization techniques [46-57], 
and image analysis methods [58-63], several microfluidic devices containing microchannels have been proposed to study RBC deformability in environments closer to in vivo microcirculation. Most of the proposed microfluidic devices to perform RBC deformability characterization can be classified as fluid-induced deformation microchannels (when the dimensions of the channels used to generate deformability are larger than the tested cells) and as structure-induced deformation microchannels (constriction channels with dimensions similar or smaller than the diameter of tested cells). To the best of our knowledge, the first application of a microfluidic constriction channel to perform RBC deformability measurements was done by Tsukada et al. [20]. In this study they measured RBC deformability of diabetic RBCs flowing through constriction microchannels and they reported that the deformability of diabetic RBCs was lower than healthy RBCs. A few years later, Shelby et al. [21] used a polydimethylsiloxane constriction microchannel to investigate the deformability changes between malaria infected RBCs and healthy RBCs. As expected, they have confirmed that the deformation of the infected RBCs decreases as the parasite progresses. After these two deformability research studies several microfluidic devices, having constriction microchannels, were proposed to measure the deformation of RBCs $[5,8,19,26,28,29,32,33,64,65]$, white blood cells (WBCs) [31,35,66], and cancer cells [22,37]. Although, the majority of the proposed microfluidic devices to perform RBC deformability characterization have focused on the strong shear effects created by the walls, these kinds of devices, due to the extremely small dimensions of the microchannels, have several critical difficulties, including fabrication complexity, flow control, and microflow visualizations. One way to overcome such experimental difficulties is by using fluid-induced deformation microfluidic devices. These kinds of devices are easier to fabricate [6] and, most of the time, produce a combination of shear and extensional flows. Some successful examples, by using abrupt or sudden contractions, are the studies performed by Zhao et al. [25], Forsyth et al. [67], and Fujiwara et al. [68]. Zhao et al. [69] have performed measurements of the RBCs' deformation in a sudden-contraction microchannel and they have reported that under different flow rates, RBC elongation reached a maximum value and could not deform any further. Forsyth et al. [67], by using a microfluidic constriction channel, have studied the deformability and dynamic behavior of both healthy and hardened RBCs and they have found different types of flow motion due to the increased shear rate in the constriction microchannel. The effect of RBCs deformability on the cell-free layer (CFL) thickness, by hardening RBCs, was also investigated at an abrupt microfluidic constriction channel by Fujiwara et al. [68]. They have found that the RBC deformability plays an important role on the asymmetry of the CFL thickness and they have reported that the motions of RBCs are strongly affected by the deformability, haematocrit, and the channel geometry. However, abrupt constriction microchannels fail to produce homogeneous extensional flows and, as a result, several researchers have been assessing RBC deformability using hyperbolic converging microchannels $[19,28-34]$. RBC deformability changes in response to shear and extensional flows strongly depend on the geometric configuration and dimensions of the constriction. For instance, the motion and deformation of a RBC passing through a sudden constriction is different from a RBC passing through a smooth or hyperbolic constriction. Pinho et al. [65] have developed a partial cell separation microfluidic device, where RBC deformability was assessed in different kinds of constriction channels. Figure 5 shows RBCs flowing through a smooth and a sudden (or abrupt) constriction microchannel.

In Figures 6 and 7 it is possible to observe the DIs and velocities of two individual RBCs flowing through a smooth and a sudden constriction microchannel, respectively. These results show that for both situations when the RBCs start to enter the constriction region the cells velocities increase and, consequently, they deform up to a maximum value. The measurements performed in a sudden contraction (see Figure 7) show that the RBCs' elongation tends to reach to a maximum value and, afterwards, do not deform any further due to the constant velocity that cells possess when they flow within the contraction. These latter results are in accordance with the findings performed by Zhao et al. [25]. However, recent results performed by Zeng and Ristenpart [69] have shown that the deformability of the RBCs tend to decrease slightly as they progress within the contraction 
region. Hence, these contradictory results show that there is a need for further research in this field. However, it is clear that the RBCs flowing through this kind of contraction are not subjected to constant strain rates. This is in contrast to the flow phenomenon that happens in hyperbolic contractions.
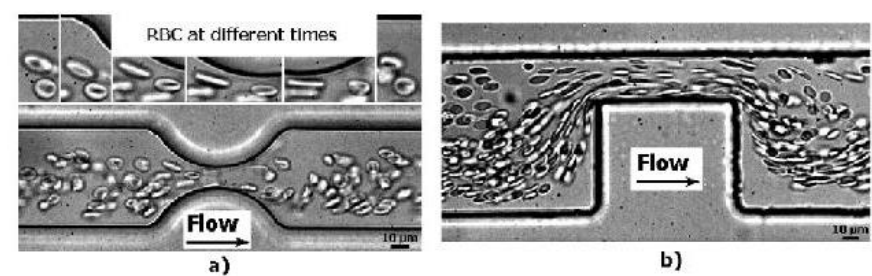

b)

Figure 5. RBCs flowing through a microchannel with (a) a smooth and (b) a sudden (or abrupt) contraction (adapted from [65]).

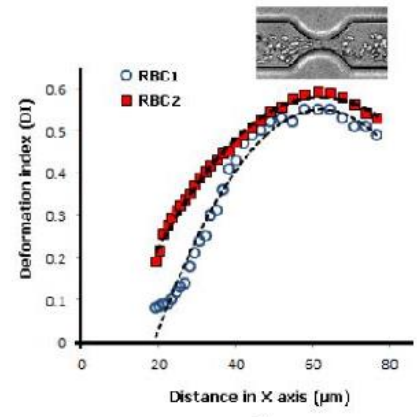

a)

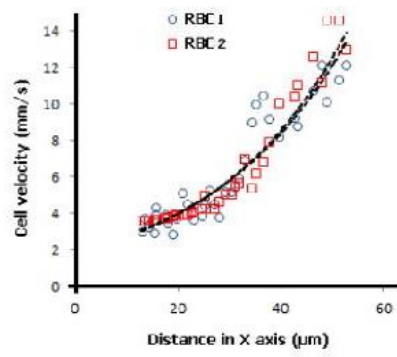

b)

Figure 6. Individual RBCs (a) DI and (b) velocity flowing through a smooth contraction microchannel for the same flow rate. The $\mathrm{X}$ axis correspond to the main flow direction.

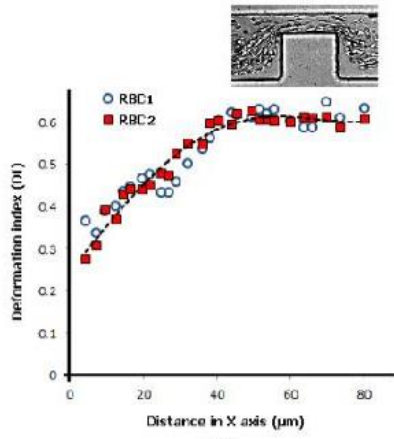

a)

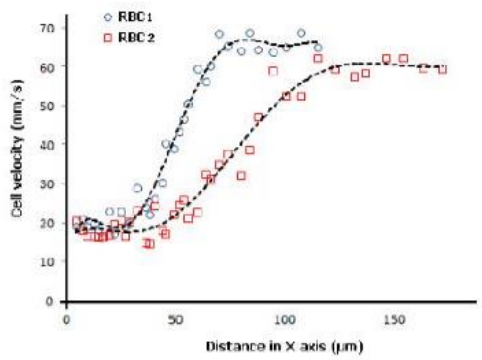

b)

Figure 7. Individual RBCs (a) DI and (b) velocity flowing through a sudden contraction microchannel for the same flow rate. The $\mathrm{X}$ axis correspond to the main flow direction.

It is known that RBCs' rigidity has been correlated with malaria, sickle cell disease, diabetes mellitus, and others haematological disorders and diseases that affect RBC deformability $[1,2,4]$. Therefore, several flow studies with rigid RBCs $[19,67]$, or with microparticles that simulate rigid RBCs, have been investigated due to the important role that they play in clarifying the hemodynamic behavior of 
diseased cells in microcirculation. Pinho et al. [70] have performed a study in order to clarify the flow behavior of both healthy RBCs and rigid microparticles when subjected to high shear rates. In this study, they have investigated the trajectories and DI in a microchannel with a pronounced microstenosis (75\%). By using a microfluidic device fabricated by a soft lithography technique, they have used a solution of Dextran 40 containing a mixture of $0.5 \%$ polystyrene (PS) latex microspheres $(10 \mu \mathrm{m})$, that mimic rigid RBCs (arRBCs) mixed with $1 \%$ of healthy ovine RBCs (diameter: $\sim 5 \mu \mathrm{m}$ ). The in vitro experiments were performed under different flow rates $(1,10,20 \mu \mathrm{L} / \mathrm{min})$ and the DI of both arRBCs and healthy RBCs were measured and compared. More detailed information about the experimental setup can be found elsewhere [70].

In Figure 8 it is shown that, for both RBCs (rigid and healthy), the maximum DI was obtained at the highest flow rate used in this study and within the stenosis region (represented by Sections 2-4). As expected, healthy RBCs had higher DIs when compared with rigid microparticles (arRBCs). In addition, it was at the highest flow rate of $20 \mu \mathrm{L} / \mathrm{min}$ that healthy RBCs obtaining a maximum DI of 0.38 in comparison to the 0.09 obtained by the arRBCs. These results are consistent with the ones obtained by Pinho et al. [65], where healthy human RBCs were investigated using different kinds of constrictions. Additionally, in this study, they have observed that some of the ovine RBCs have changed their normal shape to a parachute or umbrella shape when passing through the sudden constriction microchannel. In contrast, the rigid microparticles did not exhibit any noteworthy change from their original shape. Note that the measured residual values of the arRBC DIs were mainly due to image distortions of the high-speed microparticles.

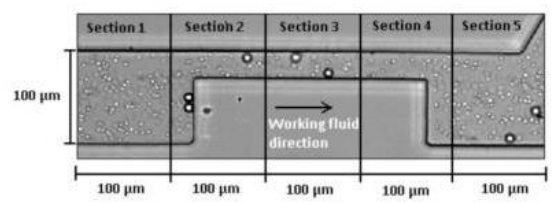

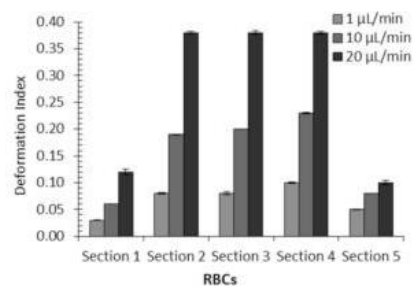

(a)

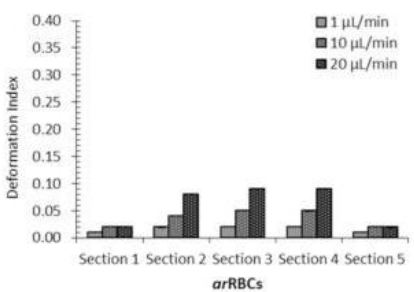

(b)

Figure 8. DI measured at five different sections of the stenosed microchannel for different flow rates: (a) healthy ovine RBCs; and (b) particles mimicking rigid RBCs (arRBCs). Error bars represent a 95\% confidence interval (adapted from [70]).

\subsection{Deformability in Rectangular PDMS Microcapillaries and Micropillars}

Although it is difficult to fabricate and control the flow in constriction microchannels with dimensions similar to RBC diameters, this kind of geometry is one the most popular ways to measure the deformability of RBCs. As it is possible to observe in Figure 9, RBCs flowing through structure-induced deformation microchannels, the RBCs tend to deform into a parachute shape or umbrella shape. Researchers, such as Tsukada et al. [20], Jeong et al. [39], and Tomaiuolo et al. [26], have calculated the RBCs' deformability by applying the formula $\mathrm{L} / \mathrm{D}$, where $\mathrm{L}$ and $\mathrm{D}$ represent the length and diameter of a deformed RBC, respectively (see Figure 9). Note that, in the present study, this measurement approach is designated as the deformation ratio (DR). By following this approach, we have analyzed and measured the DR of two individual RBCs flowing through a structure-induced deformation microchannel (see Figure 10). 

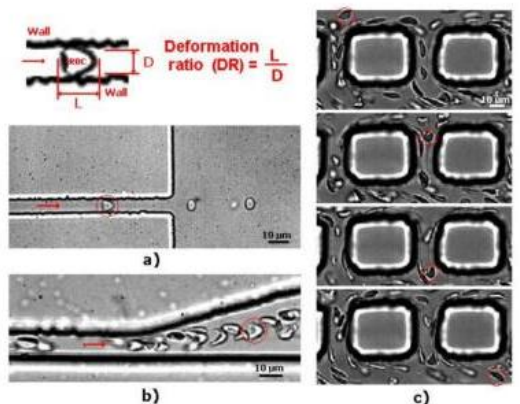

Figure 9. RBCs flowing through (a) rectangular PDMS microcapillary (b) divergent region upstream of a rectangular PDMS microcapillary; and (c) micropillars, adapted from [31].

Figure 10 shows the DRs and correspondent velocities of two individual RBCs flowing through a microchannel with dimensions similar to the RBC diameter. The results clearly show an abrupt decrease of both DRs and velocities when the RBCs leave the constriction and enter into an expansion region. It is worth mentioning that as soon as the RBC leaves the constriction region, the RBC changes from a parachute to a nearly circular shape. However, this latter behavior is not always true as it is possible to visualize in Figure 9. In Figure 9a, due to the low local haematocrit and abrupt expansion when the RBC leaves the constriction region, the RBC changes its shape to a circle. In contrast, when the RBC flows within a high local haematocrit and smooth expansion, the RBC tends to keep its parachute shape for a certain period of time. Eventually, when the shear stress induced by the walls decrease the $\mathrm{RBC}$ tend to change to a nearly circular shape. Figure $9 \mathrm{c}$ shows that, besides the effect of the geometry and local haematocrit, the orientation is also a parameter that plays an important role on the RBC deformability. Although several numerical blood flow studies [71-78] have been proposed to better understand the RBCs' flow behavior in microchannels and microvessels, our understanding of the $\mathrm{RBC}$ motion, orientation, and deformability at the microcirculation level is still far from complete.

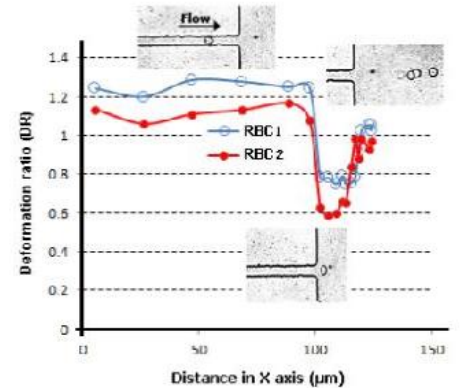

a)

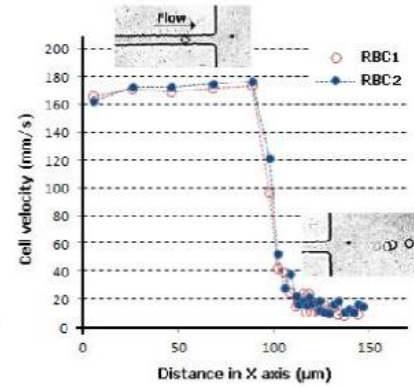

b)

Figure 10. Individual RBCs' (a) DR and (b) velocity flowing through a rectangular PDMS microcapillary for the same flow rate. The $\mathrm{X}$ axis corresponds to the main flow direction.

\subsection{Comparison of Cells' Deformability Studies}

Table 2 shows a summary comparing the main features of several cells deformability studies performed in microfluidic devices. Representative features for comparison are the microfluidic technique, blood cell types, main flow phenomenon and the used approach to measure the degree of deformability of the cells. 


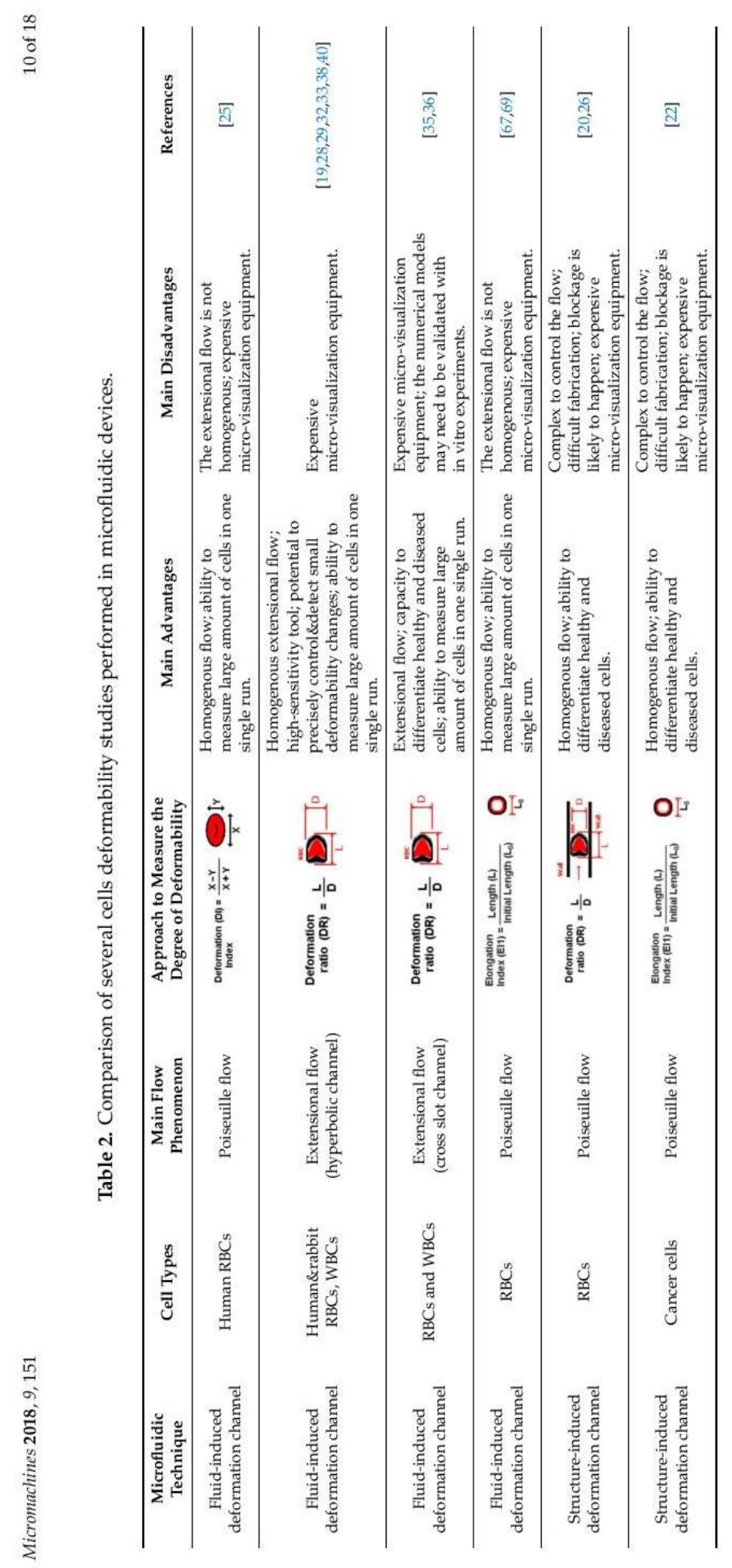




\section{Deformation of Bubbles and Droplets}

The deformation of a gas bubble in a simple shear flow depends on the viscosity ratio, $\lambda$, and on the capillary number, $\mathrm{Ca}[79]$.

The viscosity ratio is defined as:

$$
\lambda=\frac{\mu_{g}}{\mu_{l}}
$$

where $\mu_{g}$ is the gas viscosity and $\mu_{l}$ the viscosity of the liquid.

The capillary number is defined as:

$$
C a=\frac{W \dot{\gamma} \mu_{l}}{\sigma}
$$

where $\sigma$ is the surface tension, $\mathrm{W}$ the characteristic dimension of the flow and $\dot{\gamma}$ the flow shear rate. For $C a \ll 1$ and $\lambda \ll 1$, the deformation is very small and changes linearly with the capillary number.

Müller-Fischer et al. [79] have analyzed the deformation and breakup of bubbles in a parallel band apparatus to understand the influence of the viscosity ratio and the capillary number. The size of the bubbles was approximately $1 \mathrm{~mm}$. The bubbles were subject to deformation under a simple shear flow. By increasing the capillary number, deformation indices of about 0.9 were obtained. As a result, an empirical relation for the deformation index versus the capillary number was obtained and compared with correlations from the literature. Anderl et al. [80] developed a numerical method to predict the deformation of bubbles and were able to predict the results of Müller-Fischer et al. [79]. Wei et al. [81] have used the Lattice Boltzmann method to simulate the deformation of a bubble and were able to correctly predict the shape of the bubble under a simple shear flow.

Bubble deformation has also been studied in T-junction divergent flows. Fu et al. [82] identified three types of symmetric breakup of bubbles. The first one was controlled by the pressure increase in the liquid phase and the second type was controlled by the pressure increase and viscous effects. In the third type a scaling law for the minimum neck was observed. During the experiments, non-breakup bubbles were observed. As a result, the authors have proposed phase diagrams (capillary number versus normalized bubble length) showing the different conditions to observe various types of bubble behavior. Liu et al. [83] have studied this flow by numerical methods, obtaining detailed velocity and pressure fields, in addition to the bubble shapes and breakup conditions. These authors observed breakup regimes similar to the ones observed by Fu et al. [82].

In microvessels, the flow of air microbubbles may block arterioles and capillaries and, as a result, may stop the supply of blood to certain regions of the human body. Pathological events caused by microbubbles trapped in blood vessels need to be better understood. The shape and velocity of microbubbles in microchannels is known to be dependent on the capillary number and Reynolds number [84]. However, the blood cells present an additional complication. Hence, it is important to improve our understanding of the motion and deformation of microbubbles flowing in microchannels with dimensions similar to in vivo microvessels. A microfluidic system capable of generating air microbubbles was used to investigate the effect of a constriction microchannel on the deformation of individual air microbubbles flowing within in vitro blood. The fabrication technique of a flow-focusing device and the flow conditions of in vitro blood containing microbubbles are presented and discussed in more detail elsewhere $[85,86]$. Briefly, the microbubbles were produced in the following way: the dispersed phase (air) was squeezed by two counter-streaming blood flows of the carrier phase, forcing the gas to break up and, consequently, the bubbles were generated. Two kinds of bubbles were observed in this microfluidic device: Taylor bubbles and spherical bubbles (Figure 11). The-Taylor bubbles were formed and have preserved their shape until they reach the smooth expansion, where they acquired a circular shape. These Taylor bubbles flow through the microfluidic channel separated from each other by liquid slugs and from the wall by a thin liquid film. As expected, the bubbles flowing through the contraction region have higher velocities when compared with the spherical bubbles 
flowing within the expansion region of the device. Note that the formation of large bubbles in the expansion region of the microchannel was also observed. These latter bubbles are formed mainly due to the collision between them, which led to coalescence. Another point of interest is not only the formation of a cell-free layer around the bubbles, but also the effect of bubbles on the variation of the local hematocrit. This phenomenon is presented and discussed in more detail elsewhere [85].

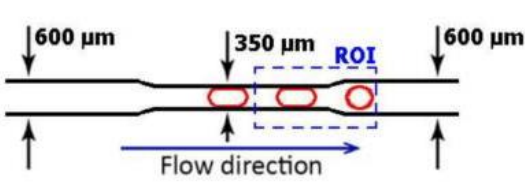

(a)

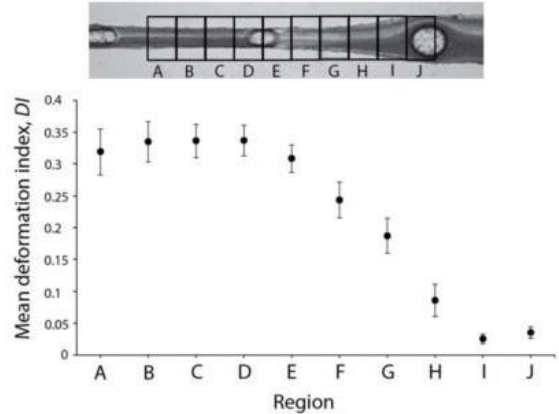

(b)

Figure 11. (a) Schematic representation of the microchannel contraction region and flow direction of a device to study gas embolisms [85]. The region of interest is indicated by a dotted rectangle; and (b) the mean deformation index of bubble flowing through the contraction region.

Figure 11 shows the transition of the DI of a microbubble flowing through the contraction region. Eight individual microbubbles were measured frame by frame and DI values were averaged for 10 different regions (A-J) along the microchannel. Around Region A, the slug bubble is generated. From Region B to D, the DI values are approximately constant, and when the bubble approaches the expansion region it starts decreasing. At Regions I and J, the bubble shapes correspond to an almost perfect circle and tends to keep its shape through this region.

Overall, it is possible to observe certain similar features when the air bubbles and RBCs leave the contraction and enter the expansion region. However, it is also clear that, at the contraction region, the bubbles' deformability shape significantly differ from the RBCs. For instance, in these experiments the air bubbles do not deform into a parachute shape as it is possible to observe with the RBCs. The capillary number of the experiments is $1.4 \times 10^{-4}$. Bubbles in this range of capillary numbers $(\mathrm{C} a<0.05)$ occupy almost all the available channel and do not deform [84].

An overview of the methods used to study bubble and drop deformation is presented in Table 3. Some techniques usually applied to bubbles, such as the imposition of shear flow by moving plates and the use of T-junction divergent flows, have also been applied to drops $[87,88]$. Hoang et al. [87] have used stop-flow numerical simulations to study the deformation and breakup of droplets in a T-junction divergent flow. They have identified two breakup phases, the first was the deformation dependent on the external flow and, the second, a surface tension-driven rapid pinching leading to breakup. Additionally, drops have been studied in a cross-slot divergent flow in a microfluidic device [89]. The deformation of the drop was dependent on $\mathrm{Ca} \cdot \delta^{2}$, where $\mathrm{Ca}$ is the capillary number and $\delta$ is a confinement parameter equal to the ratio between the drop size and the microchannel depth. Hyperbolic contractions have also been used to study not only the deformation of drops in systems with different surfactants [89], but also the deformation and breakup of Pickering droplets [86]. In this configuration the drop deformation depends on the capillary number and on the confinement parameter. 
Table 3. Comparison of several deformability studies performed in microfluidic devices for bubbles and droplets.

\begin{tabular}{|c|c|c|c|c|}
\hline $\begin{array}{l}\text { Microfluidic } \\
\text { Technique }\end{array}$ & Fluid & $\begin{array}{l}\text { Main Flow } \\
\text { Phenomenon }\end{array}$ & Configuration & References \\
\hline $\begin{array}{c}\text { parallel band/plate } \\
\text { apparatus }\end{array}$ & Bubbles and drops & Shear flow & & {$[79,80,88]$} \\
\hline T-junction divergence & Bubbles and drops & Extensional flow & & {$[82,83,87]$} \\
\hline $\begin{array}{c}\text { Fluid-induced } \\
\text { deformation channel }\end{array}$ & Drops & $\begin{array}{c}\text { Extensional flow } \\
\text { (hyperbolic channel) }\end{array}$ & & {$[90,91]$} \\
\hline $\begin{array}{c}\text { Fluid-induced } \\
\text { deformation channel }\end{array}$ & Drops & Extensional flow & 00 & [89] \\
\hline
\end{tabular}

\section{Conclusions and Future Directions}

Microfluidic devices have the advantage of being suitable to deal with single-cell deformability while testing large numbers of cells in one single run. This high throughput ability, together with the ability to achieve a controlled flow, make it possible to detect small changes in RBC deformability in a more efficient and less time-consuming way when compared with other deformability measurement techniques, such as micropipette aspiration, rheoscope, and optical tweezers. This review has shown RBC deformability measurements at both fluid- and structure-induced deformation microfluidic devices. Hence, visualizations and measurements of the deformation of RBCs flowing through hyperbolic, smooth, and sudden-contraction microchannels were investigated and compared. Our comparative results show that RBCs flowing through a hyperbolic contraction experience a strong extensional flow with a region of homogeneous strain rate along the centerline. Hence, hyperbolic-shaped microchannels have shown the potential to precisely control and detect small changes in RBC deformability in pathological situations. A recent haemocompatibility study of RBCs in contact with nanoparticles, has shown that these kinds of microfluidic devices were able to detect small changes of RBC deformability where traditional biocompatibility tests did not show any influence [32]. In conclusion, the hyperbolic-shaped constriction microchannels could be a promising tool to perform sensitive cell deformability measurements and, consequently, to be used as a clinical tool for early detection and diagnosis of blood diseases. However, this technique still facing many challenges, such as the use of low-cost micro-visualization equipment to quantitatively measure the RBC deformability and the development of fast and reliable image analysis methods able to measure both RBC motion and deformation in an automatic manner.

PDMS microfluidic devices have also proved to be an extremely powerful method to better understand the effect of the flowing air microbubbles on several blood flow phenomena happening at the micro-scale level. Our flow measurements and visualizations have shown that the microbubbles 
promote the formation of a cell-free layer around it and, as a consequence, the local haematocrit was affected. In the near future we plan to compare the obtained experimental in vitro results with multi-phase numerical models to better understand the effect of the air microbubbles on the blood flow behavior in microchannels and microvessels.

Acknowledgments: The authors acknowledge the financial support provided by the project POCI-01-0145FEDER-016861 (with associated reference PTDC/QEQ-FTT/4287/2014) from FCT (Foundation for Science and Technology) and FEDER. This work was also funded by the ERDF-European Regional Development Fund through the COMPETE Programme (operational programme for competitiveness) and by National Funds through the FCT within projects and UID/EMS/00532/2013, UID/EMS/04077/2013 and the projects POCI-01-0145-FEDER-007043, POCI-01-0145-FEDER-006961, UID/CEC/00319/2013. David Bento, Raquel O. Rodrigues, Diana Pinho, and Vera Faustino, acknowledge, respectively, the PhD scholarships SFRH/BD/91192/2012, SFRH/BD/97658/2013, SFRH/BD/89077/2012, and SFRH/BD/99696/2014, and Rui Lima acknowledge fellowship SFRH/BSAB/135419/2017, all granted by FCT.

Author Contributions: David Bento, Raquel O. Rodrigues, Diana Pinho and Vera Faustino contributed with the data compilation and writing of the manuscript. Carla S. Fernandes, Ana I. Pereira, Valdemar Garcia contributed in the revision of the manuscript. João M. Miranda and Rui Lima contributed in the design and execution of the article, writing and revision of the manuscript.

Conflicts of Interest: The authors declare no conflict of interest.

\section{References}

1. Mokken, F.C.; Kedaria, M.; Henny, C.P.; Hardeman, M.R.; Gelb, A.W. The clinical importance of erythrocyte deformability, a hemorrheological parameter. Ann. Hematol. 1992, 64, 113-122. [CrossRef] [PubMed]

2. Lee, G.Y.; Lim, C.T. Biomechanics approaches to studying human diseases. Trends Biotechnol. 2007, 25, 111-118. [CrossRef] [PubMed]

3. Musielak, M. Red blood cell deformability measurement: Review of techniques. Clin. Hemorheol. Microcirc. 2009, 42, 47-64. [PubMed]

4. Kim, Y.; Kim, K.; Park, Y. Measurement techniques for red blood cell deformability: Recent advances. In Blood Cell-An Overview of Studies in Hematology; InTech: London, UK, 2012.

5. Manouk, A.; Magalie, F.; Renita, H.; Kristian, S.; Catherine, A.B.-P.; Howard, A.S. Cellular-scale hydrodynamics. Biomed. Mater. 2008, 3, 034011.

6. Faustino, V.; Catarino, S.O.; Lima, R.; Minas, G. Biomedical microfluidic devices by using low-cost fabrication techniques: A review. J. Biomech. 2016, 49, 2280-2292. [CrossRef] [PubMed]

7. Zheng, Y.; Nguyen, J.; Wei, Y.; Sun, Y. Recent advances in microfluidic techniques for single-cell biophysical characterization. Lab Chip 2013, 13, 2464-2483. [CrossRef] [PubMed]

8. Tomaiuolo, G. Biomechanical properties of red blood cells in health and disease towards microfluidics. Biomicrofluidics 2014, 8, 051501. [CrossRef]

9. Xue, C.; Wang, J.; Zhao, Y.; Chen, D.; Yue, W.; Chen, J. Constriction channel based single-cell mechanical property characterization. Micromachines 2015, 6, 1794-1804. [CrossRef]

10. Nash, G.; O'Brien, E.; Gordon-Smith, E.; Dormandy, J. Abnormalities in the mechanical properties of red blood cells caused by plasmodium falciparum. Blood 1989, 74, 855-861. [PubMed]

11. Paulitschke, M.; Nash, G. Membrane rigidity of red blood cells parasitized by different strains of plasmodium falciparum. J. Lab. Clin. Med. 1993, 122, 581-589. [PubMed]

12. Glenister, F.K.; Coppel, R.L.; Cowman, A.F.; Mohandas, N.; Cooke, B.M. Contribution of parasite proteins to altered mechanical properties of malaria-infected red blood cells. Blood 2002, 99, 1060-1063. [CrossRef] [PubMed]

13. Suresh, S.; Spatz, J.; Mills, J.; Micoulet, A.; Dao, M.; Lim, C.; Beil, M.; Seufferlein, T. Connections between single-cell biomechanics and human disease states: Gastrointestinal cancer and malaria. Acta Biomater. 2005, 1, 15-30. [CrossRef] [PubMed]

14. Agrawal, R.; Smart, T.; Nobre-Cardoso, J.; Richards, C.; Bhatnagar, R.; Tufail, A.; Shima, D.; Jones, P.H.; Pavesio, C. Assessment of red blood cell deformability in type 2 diabetes mellitus and diabetic retinopathy by dual optical tweezers stretching technique. Sci. Rep. 2016, 6, 15873. [CrossRef] [PubMed] 
15. Dulińska, I.; Targosz, M.; Strojny, W.; Lekka, M.; Czuba, P.; Balwierz, W.; Szymoński, M. Stiffness of normal and pathological erythrocytes studied by means of atomic force microscopy. J. Biochem. Biophys. Methods 2006, 66, 1-11. [CrossRef] [PubMed]

16. Fornal, M.; Lekka, M.; Pyka-Fościak, G.; Lebed, K.; Grodzicki, T.; Wizner, B.; Styczeń, J. Erythrocyte stiffness in diabetes mellitus studied with atomic force microscope. Clin. Hemorheol. Microcirc. 2006, 35, $273-276$. [PubMed]

17. Maciaszek, J.L.; Lykotrafitis, G. Sickle cell trait human erythrocytes are significantly stiffer than normal. J. Biomech. 2011, 44, 657-661. [CrossRef] [PubMed]

18. Shin, S.; Ku, Y.-H.; Ho, J.-X.; Kim, Y.-K.; Suh, J.-S.; Singh, M. Progressive impairment of erythrocyte deformability as indicator of microangiopathy in type 2 diabetes mellitus. Clin. Hemorheol. Microcirc. 2007, 36, $253-261$. [PubMed]

19. Pinho, D.; Campo-Deaño, L.; Lima, R.; Pinho, F.T. In vitro particulate analogue fluids for experimental studies of rheological and hemorheological behavior of glucose-rich RBC suspensions. Biomicrofluidics 2017, 11, 054105. [CrossRef] [PubMed]

20. Tsukada, K.; Sekizuka, E.; Oshio, C.; Minamitani, H. Direct measurement of erythrocyte deformability in diabetes mellitus with a transparent microchannel capillary model and high-speed video camera system. Microvasc. Res. 2001, 61, 231-239. [CrossRef] [PubMed]

21. Shelby, J.P.; White, J.; Ganesan, K.; Rathod, P.K.; Chiu, D.T. A microfluidic model for single-cell capillary obstruction by plasmodium falciparum-infected erythrocytes. Proc. Natl. Acad. Sci. USA 2003, 100, 14618-14622. [CrossRef] [PubMed]

22. Hou, H.W.; Li, Q.; Lee, G.; Kumar, A.; Ong, C.; Lim, C.T. Deformability study of breast cancer cells using microfluidics. Biomed. Microdevices 2009, 11, 557-564. [CrossRef] [PubMed]

23. Huang, S.; Undisz, A.; Diez-Silva, M.; Bow, H.; Dao, M.; Han, J. Dynamic deformability of plasmodium falciparum-infected erythrocytes exposed to artesunate in vitro. Integr. Biol. 2013, 5, 414-422. [CrossRef] [PubMed]

24. Zeng, N.F.; Mancuso, J.E.; Zivkovic, A.M.; Smilowitz, J.T.; Ristenpart, W.D. Red blood cells from individuals with abdominal obesity or metabolic abnormalities exhibit less deformability upon entering a constriction. PLOS ONE 2016, 11, e0156070. [CrossRef] [PubMed]

25. Zhao, R.; Antaki, J.F.; Naik, T.; Bachman, T.N.; Kameneva, M.V.; Wu, Z.J. Microscopic investigation of erythrocyte deformation dynamics. Biorheology 2006, 43, 747-765. [PubMed]

26. Tomaiuolo, G.; Barra, M.; Preziosi, V.; Cassinese, A.; Rotoli, B.; Guido, S. Microfluidics analysis of red blood cell membrane viscoelasticity. Lab Chip 2011, 11, 449-454. [CrossRef] [PubMed]

27. Sousa, P.C.; Pinho, F.T.; Oliveira, M.S.; Alves, M.A. Extensional flow of blood analog solutions in microfluidic devices. Biomicrofluidics 2011, 5, 14108. [CrossRef] [PubMed]

28. Lee, S.S.; Yim, Y.; Ahn, K.H.; Lee, S.J. Extensional flow-based assessment of red blood cell deformability using hyperbolic converging microchannel. Biomed. Microdevices 2009, 11, 1021-1027. [CrossRef] [PubMed]

29. Yaginuma, T.; Oliveira, M.S.N.; Lima, R.; Ishikawa, T.; Yamaguchi, T. Human red blood cell behavior under homogeneous extensional flow in a hyperbolic-shaped microchannel. Biomicrofluidics 2013, 7, 054110. [CrossRef] [PubMed]

30. Rodrigues, R.O.; Lopes, R.; Pinho, D.; Pereira, A.I.; Garcia, V.; Gassmann, S.; Sousa, P.C.; Lima, R. In vitro blood flow and cell-free layer in hyperbolic microchannels: Visualizations and measurements. BioChip J. 2016, 10, 9-15. [CrossRef]

31. Rodrigues, R.O.; Pinho, D.; Faustino, V.; Lima, R. A simple microfluidic device for the deformability assessment of blood cells in a continuous flow. Biomed. Microdevices 2015, 17, 108. [CrossRef] [PubMed]

32. Rodrigues, R.O.; Bañobre-López, M.; Gallo, J.; Tavares, P.B.; Silva, A.M.; Lima, R.; Gomes, H.T. Haemocompatibility of iron oxide nanoparticles synthesized for theranostic applications: A high-sensitivity microfluidic tool. J. Nanopart. Res. 2016, 18, 194. [CrossRef]

33. Faustino, V.; Pinho, D.; Yaginuma, T.; Calhelha, R.C.; Ferreira, I.C.F.R.; Lima, R. Extensional flow-based microfluidic device: Deformability assessment of red blood cells in contact with tumor cells. BioChip J. 2014, 8 , 42-47. [CrossRef]

34. Calejo, J.; Pinho, D.; Galindo-Rosales, F.; Lima, R.; Campo-Deaño, L. Particulate blood analogues reproducing the erythrocytes cell-free layer in a microfluidic device containing a hyperbolic contraction. Micromachines 2016, 7, 4. [CrossRef] 
35. Gossett, D.R.; Henry, T.; Lee, S.A.; Ying, Y.; Lindgren, A.G.; Yang, O.O.; Rao, J.; Clark, A.T.; Di Carlo, D. Hydrodynamic stretching of single cells for large population mechanical phenotyping. Proc. Natl. Acad. Sci. USA 2012, 109, 7630-7635. [CrossRef] [PubMed]

36. Henon, Y.; Sheard, G.J.; Fouras, A. Erythrocyte deformation in a microfluidic cross-slot channel. RSC Adv. 2014, 4, 36079-36088. [CrossRef]

37. Guillou, L.; Dahl, J.B.; Lin, J.-M.G.; Barakat, A.I.; Husson, J.; Muller, S.J; Kumar, S. Measuring cell viscoelastic properties using a microfluidic extensional flow device. Biophys. J. 2016, 111, 2039-2050. [CrossRef] [PubMed]

38. Faustino, V.; Pinho, D.; Yaginuma, T.; Calhelha, R.; Oliveira, M.; Ferreira, I.; Lima, R. Flow of red blood cells suspensions through hyperbolic microcontractions. Visualization and simulation of complex flows in biomedical engineering. In Visualization and Simulation of Complex Flows in Biomedical Engineering Lecture Notes in Computational Vision and Biomechanics; Springer: Berlin, Germany, 2014; Volume 12, pp. 151-163.

39. Jeong, J.H.; Sugii, Y.; Minamiyama, M.; Okamoto, K. Measurement of rbc deformation and velocity in capillaries in vivo. Microvasc. Res. 2006, 71, 212-217. [CrossRef] [PubMed]

40. Yaginuma, T.; Oliveira, M.; Lima, R.; Ishikawa, T.; Yamaguchi, T. Red blood cell deformation in flows through a pdms hyperbolic microchanne. In Proceedings of the Techconnect World 2011-Microtech Conference and Expo, Boston, MA, USA, 13-16 June 2011; p. 505.

41. Muñoz-Sánchez, B.; Silva, S.; Pinho, D.; Vega, E.; Lima, R. Generation of micro-sized pdms particles by a flow focusing technique for biomicrofluidics applications. Biomicrofluidics 2016, 10, 014122. [CrossRef] [PubMed]

42. Pinho, D.; Rodrigues, R.O.; Faustino, V.; Yaginuma, T.; Exposto, J.; Lima, R. Red blood cells radial dispersion in blood flowing through microchannels: The role of temperature. J. Biomech. 2016, 49, 2293-2298. [CrossRef] [PubMed]

43. Sousa, P.C.; Pinho, F.T.; Alves, M.A.; Oliveira, M.S. A review of hemorheology: Measuring techniques and recent advances. Korea-Aust. Rheol. J. 2016, 28, 1-22. [CrossRef]

44. Wong, K.H.; Chan, J.M.; Kamm, R.D.; Tien, J. Microfluidic models of vascular functions. Ann. Rev. Biomed. Eng. 2012, 14, 205-230. [CrossRef] [PubMed]

45. Rodrigues, R.O.; Lima, R.; Gomes, H.T.; Silva, A.M. Polymer microfluidic devices: An overview of fabrication methods. U. Porto J. Eng. 2015, 1, 67-79.

46. Kim, G.B.; Lee, S.J. X-ray piv measurements of blood flows without tracer particles. Exp. Fluids 2006, 41, 195-200. [CrossRef]

47. Lima, R.; Wada, S.; Tsubota, K.-i.; Yamaguchi, T. Confocal micro-piv measurements of three-dimensional profiles of cell suspension flow in a square microchannel. Measur. Sci. Technol. 2006, 17, 797-808. [CrossRef]

48. Vennemann, P.; Kiger, K.T.; Lindken, R.; Groenendijk, B.C.; Stekelenburg-de Vos, S.; ten Hagen, T.L.; Ursem, N.T.; Poelmann, R.E.; Westerweel, J.; Hierck, B.P. In vivo micro particle image velocimetry measurements of blood-plasma in the embryonic avian heart. J. Biomech. 2006, 39, 1191-1200. [CrossRef] [PubMed]

49. Lima, R.; Wada, S.; Tanaka, S.; Takeda, M.; Ishikawa, T.; Tsubota, K.; Imai, Y.; Yamaguchi, T. In vitro blood flow in a rectangular pdms microchannel: Experimental observations using a confocal micro-PIV system. Biomed. Microdevices 2008, 10, 153-167. [CrossRef] [PubMed]

50. Lima, R.; Ishikawa, T.; Imai, Y.; Takeda, M.; Wada, S.; Yamaguchi, T. Measurement of individual red blood cell motions under high hematocrit conditions using a confocal micro-PTV system. Ann. Biomed. Eng. 2009, 37, 1546-1559. [CrossRef] [PubMed]

51. Williams, S.J.; Park, C.; Wereley, S.T. Advances and applications on microfluidic velocimetry techniques. Microfluid. Nanofluid. 2010, 8, 709-726. [CrossRef]

52. Kikuchi, K.; Mochizuki, O. Micro-piv (micro particle image velocimetry) visualization of red blood cells (rbcs) sucked by a female mosquito. Measur. Sci. Technol. 2011, 22, 064002. [CrossRef]

53. Garcia, V.; Dias, R.; Lima, R. In vitro blood flow behaviour in microchannels with simple and complex geometries. In Applied Biological Engineering-Principles and Practice; InTech: London, UK, 2012.

54. Lima, R.; Ishikawa, T.; Imai, Y.; Yamaguchi, T. Blood flow behavior in microchannels: Past, current and future trends. Single Two-Phase Flows Chem. Biomed. Eng. 2012, 513-547.

55. Pitts, K.; Mehri, R.; Mavriplis, C.; Fenech, M. Micro-particle image velocimetry measurement of blood flow: Validation and analysis of data pre-processing and processing methods. Measur. Sci. Technol. 2012, 23, 105302. [CrossRef] 
56. Sackmann, E.K.; Fulton, A.L.; Beebe, D.J. The present and future role of microfluidics in biomedical research. Nature 2014, 507, 181. [CrossRef] [PubMed]

57. Stauber, H.; Waisman, D.; Korin, N.; Sznitman, J. Red blood cell dynamics in biomimetic microfluidic networks of pulmonary alveolar capillaries. Biomicrofluidics 2017, 11, 014103. [CrossRef] [PubMed]

58. Kim, S.; Kong, R.L.; Popel, A.S.; Intaglietta, M.; Johnson, P.C. A computer-based method for determination of the cell-free layer width in microcirculation. Microcirculation 2006, 13, 199-207. [CrossRef] [PubMed]

59. Pinho, D.; Lima, R.; Pereira, A.I.; Gayubo, F. Automatic tracking of labeled red blood cells in microchannels. Int. J. Numer. Methods Biomed. Eng. 2013, 29, 977-987. [CrossRef] [PubMed]

60. Chenouard, N.; Smal, I.; De Chaumont, F.; Maška, M.; Sbalzarini, I.F.; Gong, Y.; Cardinale, J.; Carthel, C.; Coraluppi, S.; Winter, M. Objective comparison of particle tracking methods. Nat. Methods 2014, 11, 281. [CrossRef] [PubMed]

61. Taboada, B.; Monteiro, F.; Lima, R. Automatic tracking and deformation measurements of red blood cells flowing through a microchannel with a microstenosis: The keyhole model. Comput. Methods Biomech. Biomed. Eng. Imaging Vis. 2016, 4, 229-237. [CrossRef]

62. Bento, D.; Pereira, A.; Lima, J.; Miranda, J.; Lima, R. Cell-free layer measurements of in vitro blood flow in a microfluidic network: An automatic and manual approach. Comput. Methods Biomech. Biomed. Eng. Imaging Vis. 2017, 1-9. [CrossRef]

63. Mehri, R.; Niazi, E.; Mavriplis, C.; Fenech, M. An automated method for dynamic red blood cell aggregate detection in microfluidic flow. Physiol. Meas. 2018, 39, 01NT02. [CrossRef] [PubMed]

64. Forsyth, A.M.; Wan, J.; Owrutsky, P.D.; Abkarian, M.; Stone, H.A. Multiscale approach to link red blood cell dynamics, shear viscosity, and atp release. Proc. Natl. Acad. Sci. USA 2011, 108, 10986-10991. [CrossRef] [PubMed]

65. Pinho, D.; Yaginuma, T.; Lima, R. A microfluidic device for partial cell separation and deformability assessment. BioChip J. 2013, 7, 367-374. [CrossRef]

66. Rosenbluth, M.J.; Lam, W.A.; Fletcher, D.A. Analyzing cell mechanics in hematologic diseases with microfluidic biophysical flow cytometry. Lab Chip 2008, 8, 1062-1070. [CrossRef] [PubMed]

67. Forsyth, A.M.; Wan, J.; Ristenpart, W.D.; Stone, H.A. The dynamic behavior of chemically "stiffened" red blood cells in microchannel flows. Microvasc. Res. 2010, 80, 37-43. [CrossRef] [PubMed]

68. Fujiwara, H.; Ishikawa, T.; Lima, R.; Matsuki, N.; Imai, Y.; Kaji, H.; Nishizawa, M.; Yamaguchi, T. Red blood cell motions in high-hematocrit blood flowing through a stenosed microchannel. J. Biomech. 2009, 42, 838-843. [CrossRef] [PubMed]

69. Zeng, N.F.; Ristenpart, W.D. Mechanical response of red blood cells entering a constriction. Biomicrofluidics 2014, 8, 064123. [CrossRef] [PubMed]

70. Pinho, D.; Rodrigues, R.O.; Yaginuma, T.; Faustino, V.; Bento, D.; Fernandes, C.S.; Garcia, V.; Pereira, A.I.; Lima, R. Motion of rigid particles flowing in a microfluidic device with a pronounced stenosis: Trajectories and deformation index. In Proceedings of the 11th World Congress on Computational Mechanics, 5th European Conference on Computational Mechanics and 6th European Conference on Computational Fluid Dynamics, Barcelona, Spain, 20-25 July 2014; pp. 6234-6240.

71. Yamaguchi, T.; Ishikawa, T.; Tsubota, K.; Imai, Y.; Nakamura, M.; Fukui, T. Computational blood flow analysis-New trends and methods. J. Biomech. Sci. Eng. 2006, 1, 29-50. [CrossRef]

72. Lima, R.; Fernandes, C.S.; Dias, R.; Ishikawa, T.; Imai, Y.; Yamaguchi, T. Microscale flow dynamics of red blood cells in microchannels: An experimental and numerical analysis. In Computational Vision and Medical Image Processing; Springer: Dordrecht, The Netherlands, 2009; pp. 203-220.

73. Nakamura, M.; Bessho, S.; Wada, S. Spring-network-based model of a red blood cell for simulating mesoscopic blood flow. Int. J. Numer. Methods Biomed. Eng. 2013, 29, 114-128. [CrossRef] [PubMed]

74. Bento, D.; Lima, R.; M Miranda, J. Computation of a three-dimensional flow in a square microchannel: A comparison between a particle method and a finite volume method. Micro Nanosyst. 2015, 7, 142-147. [CrossRef]

75. Omori, T.; Imai, Y.; Kikuchi, K.; Ishikawa, T.; Yamaguchi, T. Hemodynamics in the microcirculation and in microfluidics. Ann. Biomed. Eng. 2015, 43, 238-257. [CrossRef] [PubMed]

76. Gambaruto, A.M. Flow structures and red blood cell dynamics in arteriole of dilated or constricted cross section. J. Biomech. 2016, 49, 2229-2240. [CrossRef] [PubMed] 
77. Imai, Y.; Omori, T.; Shimogonya, Y.; Yamaguchi, T.; Ishikawa, T. Numerical methods for simulating blood flow at macro, micro, and multi scales. J. Biomech. 2016, 49, 2221-2228. [CrossRef] [PubMed]

78. Ye, T.; Phan-Thien, N.; Lim, C.T. Particle-based simulations of red blood cells-A review. J. Biomech. 2016, 49, 2255-2266. [CrossRef] [PubMed]

79. Müller-Fischer, N.; Tobler, P.; Dressler, M.; Fischer, P.; Windhab, E.J. Single bubble deformation and breakup in simple shear flow. Exp. Fluids 2008, 45, 917-926. [CrossRef]

80. Anderl, D.; Bauer, M.; Rauh, C.; Rüde, U.; Delgado, A. Numerical simulation of bubbles in shear flow. PAMM 2014, 14, 667-668. [CrossRef]

81. Wei, Y.k.; Qian, Y.; Xu, H. Lattice boltzmann simulations of single bubble deformation and breakup in a shear flow. J. Comput. Multiph. Flows 2012, 4,111-117. [CrossRef]

82. Fu, T.; Ma, Y.; Funfschilling, D.; Li, H.Z. Dynamics of bubble breakup in a microfluidic T-junction divergence. Chem. Eng. Sci. 2011, 66, 4184-4195. [CrossRef]

83. Liu, X.; Zhang, C.; Yu, W.; Deng, Z.; Chen, Y. Bubble breakup in a microfluidic T-junction. Sci. Bull. 2016, 61, 811-824. [CrossRef]

84. Rocha, L.A.M.; Miranda, J.M.; Campos, J.B.L.M. Wide range simulation study of taylor bubbles in circular milli and microchannels. Micromachines 2017, 8, 154. [CrossRef]

85. Bento, D.; Sousa, L.; Yaginuma, T.; Garcia, V.; Lima, R.; Miranda, J.M. Microbubble moving in blood flow in microchannels: Effect on the cell-free layer and cell local concentration. Biomed. Microdevices 2017, 19, 6. [CrossRef] [PubMed]

86. Pinto, E.; Faustino, V.; Rodrigues, R.; Pinho, D.; Garcia, V.; Miranda, J.; Lima, R. A rapid and low-cost nonlithographic method to fabricate biomedical microdevices for blood flow analysis. Micromachines 2014, 6 , 121-135. [CrossRef]

87. Hoang, D.; Portela, L.; Kleijn, C.; Kreutzer, M.; Van Steijn, V. Dynamics of droplet breakup in a T-junction. J. Fluid Mech. 2013, 717. [CrossRef]

88. Sibillo, V.; Pasquariello, G.; Simeone, M.; Cristini, V.; Guido, S. Drop deformation in microconfined shear flow. Phys. Rev. Lett. 2006, 97, 054502. [CrossRef] [PubMed]

89. Ulloa, C.; Ahumada, A.; Cordero, M.L. Effect of confinement on the deformation of microfluidic drops. Phys. Rev. E 2014, 89, 033004. [CrossRef] [PubMed]

90. Mulligan, M.K.; Rothstein, J.P. Deformation and breakup of micro-and nanoparticle stabilized droplets in microfluidic extensional flows. Langmuir 2011, 27, 9760-9768. [CrossRef] [PubMed]

91. Mulligan, M.K.; Rothstein, J.P. The effect of confinement-induced shear on drop deformation and breakup in microfluidic extensional flows. Phys. Fluids 2011, 23, 022004. [CrossRef] 


\title{
B. A simple microfluidic device for the deformability assessment of blood cells in a continuous flow
}

\section{A simple microfluidic device for the deformability assessment of blood cells in a continuous flow}

\author{
Raquel O. Rodrigues ${ }^{1,2}$ - Diana Pinho ${ }^{2,3}$ - Vera Faustino ${ }^{2,4} \cdot$ Rui Lima ${ }^{2,3,5}$
}

Published online: 19 October 2015

C. Springer Science+Business Media New York 2015

\begin{abstract}
Blood flow presents several interesting phenomena in microcirculation that can be used to develop microfluidic devices capable to promote blood cells separation and analysis in continuous flow. In the last decade there have been numerous microfluidic studies focused on the deformation of red blood cells (RBCs) flowing through geometries mimicking microvessels. In contrast, studies focusing on the deformation of white blood cells (WBCs) are scarce despite this phenomenon often happens in the microcirculation. In this work, we present a novel integrative microfluidic device able to perform continuous separation of a desired amount of blood cells, without clogging or jamming, and at the same time, capable to assess the deformation index (DI) of both WBCs and RBCs. To determine the DI of both WBCs and RBCs, a hyperbolic converging microchannel was used, as well as a suitable image analysis technique to measure the DIs of these
\end{abstract}

Electronic supplementary material The online version of this article (doi:10.1007/s10544-015-0014-2) contains supplementary material, which is available to authorized users.

$\triangle$ Rui Lima

ruimec@ipb.pt

I LCM-Laboratory of Catalysis and Materials - Associate Laboratory LSRE/LCM, Faculdade de Engenharia, da Universidade do Porto (FEUP), R. Dr. Roberto Frias, 4200-465 Porto, Portugal

2 Polytechnic Institute of Bragança, ESTiG/IPB, C. Sta. Apolónia, 5301-857 Bragança, Portugal

3 CEFT, Faculdade de Engenharia da Universidade do Porto (FEUP), R. Dr. Roberto Frias, 4200-465 Porto, Portugal

4 Center for MicroElectromechanical Systems (CMEMS-UMinho), University of Minho, Campus de Azurém, 4800-058 Guimarães, Portugal

5 Mechanical Engineering Department, University of Minho, Campus de Azurém, 4800-058 Guimarães, Portugal blood cells along the regions of interest. The results show that the WBCs have a much lower deformability than RBCs when subjected to the same in vitro flow conditions, which is directly related to their cytoskeleton and nucleus contents. The proposed strategy can be easily transformed into a simple and inexpensive diagnostic microfluidic system to simultaneously separate and assess blood cells deformability.

Keywords Microfluidic devices · Cell separation and deformability $\cdot$ Hyperbolic microchannel - Blood on chips · $\mathrm{RBC} \cdot \mathrm{WBC}$

\section{Introduction}

Blood is an extremely information-rich and easily accessible tissue, which can be used to diagnose several diseases with multiple techniques. However, due to the complex blend of cells it requires the isolation of a limited number of cells, so an accurate analysis can be realized and eventually applied to a variety of biomedical applications, such as diagnostics, therapeutics and cell biology (Gossett et al. 2010). Most of the standard techniques used for cell separation and sorting are often labour intensive or require additional external labels to identify cells (Jinlong et al. 2008; Pinho et al. 2013). For these reasons, label-free microfluidic techniques have been raising interest, since they avoid the use of biochemical labels that may change the cell properties and increase costs (Gossett et al. 2010). Therefore, intrinsic biomarkers, such as cell size, electrical polarization, density, deformability and/or hydrodynamic properties are being explored (Chen et al. 2008; Hou et al. 2010; Kim et al. 2010; Pinho et al. 2013; Shevkoplyas et al. 2005). Concerning cells separation based on their size, microstructures such as micro-pillar arrays, micro-weir and membranes with holes have been used as a simple, non- 
destructive and easy integrative step to be implemented, in order to archive a single and complete device (Bradley et al. 2012; Chen et al. 2008; Yang et al. 1999). Nevertheless, several separation microfluidic devices are based in dead-end filtration, which might lead to clogging or jamming (Agbangla et al. 2012; Chen et al. 2007; Georgieva et al. 2010). To overcome this issue, cross-flow filtration has being used (Tae Goo et al. 2014), operating under similar sizeexclusion precepts, with the fluid flowing perpendicular to the micro-pillar arrays or micro-weir filters. Thereby, the rejected cells continue to travel in the direction of primary flow, while selected cells flow into a separated outlet (Chen et al. 2008; Gossett et al. 2010). Ji and his colleagues (Ji et al. 2008) have compared four main types of silicon-based microfilters and they have found that the cross-flow filtration approach is the most efficient way of sorting RBCs and WBCs. Additionally this microfilter was the most suitable for handling blood flow avoiding problems associated with aggregations of cells and filter clogging. Recently, some research works regarding the plasma separation from whole blood (Chen et al. 2008; VanDelinder and Groisman 2006), white blood cells (WBCs) from whole blood (VanDelinder and Groisman 2007), fluorescent polystyrene beads separated by sizes (Metz et al. 2004) and neonatal rat cardiac cell population from whole blood (Murthy et al. 2006) have been reported. However, to the best of our knowledge, no study has reported until now an integrative microfluidic device, capable of separating a small amount of blood cells (WBCs and red blood cells (RBCs)) from the initial blood fluid, and at the same time, studying and detecting changes in their deformability, through the deformation index (DI) assessment of isolated individual cells. In fact, in microfluidics and cell biology fields there is a need of a better understanding of the flow behaviour and deformation of WBCs in an in vitro environment. This type of environment was already shown to be very different from the in vivo ones, where WBCs adapt their shape (possibly by deformation) penetrating through the walls of vascular endothelial cells, by activation of proinflammatory mediators (cytokines, chemokines, or bacterial peptides) (Liu and Wang 2004). Since WBCs are a set of several types of different and specialized cells, they are usually subdivided in: granulocytes (neutrophils, basophils, and eosinophils), characterized by the presence of differently staining granules in their cytoplasm or agranulocytes (lymphocytes, monocytes, and macrophages), characterized by the apparent absence of granules in their cytoplasm (Khismatullin 2009). Due to the polymorphism and complexity of these cells, they should be studied individually, in order to verify their specificities of deformability and hydrodynamic properties, such as their sizes, cytoplasmatic contents and densities. In the group of agranulocytes, peripheral blood mononuclear cells (PBMC) (i.e., lymphocyte, monocyte or macrophage) are main actors in inflammatory processes and linked to many diseases, such as rheumatoid arthritis (Tanino et al. 2009), atherosclerosis (Ortega et al. 2012), asthma (Covar et al. 2010), HIV (Fu et al. 2011), cancer (Frampton et al. 2013), among others. Moreover, they are a potential source of non-invasive biomarkers for diagnosis and monitoring of many diseases (Maes et al. 2013). In this research, we develop a pillar-type cross-flow filtration microfluidic device to separate and collect RBCs and a specific type of WBCs, the PBMC, and then perform the individual deformation assessment of them, based on the extensional flow approach. The latter methodology was adopted due to the ability of providing a detailed quantitative description of the degree of deformation of blood cells under a controlled homogeneous extensional flow field, in a region of homogeneous extension rate. Studies focusing on the extensional flow effect on the blood cells deformability are scarce, despite this phenomenon often happens in the microcirculation, particularly when there is a sudden change in geometry and consequently, there is a dramatic change of the cells velocity and deformability. Recent studies performed by Lee et al. (2009), Yaginuma et al. (2013) and Faustino et al. (2014) have investigated RBCs behaviour under the effect of a extensional flow in hyperbolic converging microchannels. However, in the literature there are few studies concerning the effect of extensional-dominated flows on the deformation degree of WBCs. Therefore, the main purpose of this study is the development of an integrative microfluidic device able to separate from an initial in vitro blood sample, a low amount of blood cells (PBMCs and $\mathrm{RBCs}$ ), and at the same time, evaluate their deformability through a hyperbolic converging microchannel.

\section{Materials and methods}

\subsection{Microfluidic device design and fabrication}

The microfluidic device used in the present work was designed to include a cross-flow filtration barrier to separate a low amount of WBCs (i.e., PBMCs) and RBCs from an in vitro blood sample, as well as several sequences of hyperbolic channels, in the outlet of the sub-channels, to perform deformability assessment on the separated blood cells. A schematic view of the microfluidic device with dimensions of the main channel, $55 \times 500 \times 17,482 \mu \mathrm{m}$ (height $\times$ width $\times$ length), is shown in Fig. 1. The pillar-type filtration barrier was set with a total of 42 rectangular pillars with dimensions of $55 \times$ $50 \mu \mathrm{m}$ (width $\times$ length), spaced by $10 \mu \mathrm{m}$, and the triplicate sequences of the hyperbolics were set in the outlet of the four sub-channels, with dimension of $55 \times 20 \times 383 \mu \mathrm{m}$ (height $\times$ width $\times$ length). To perform the deformability assessment hyperbolic converging microchannels were designed with $382 \mu \mathrm{m}$ of length, as well as widths of 400 and $20 \mu \mathrm{m}$ at the wide and narrow sizes, respectively, corresponding to

\section{Q Springer}




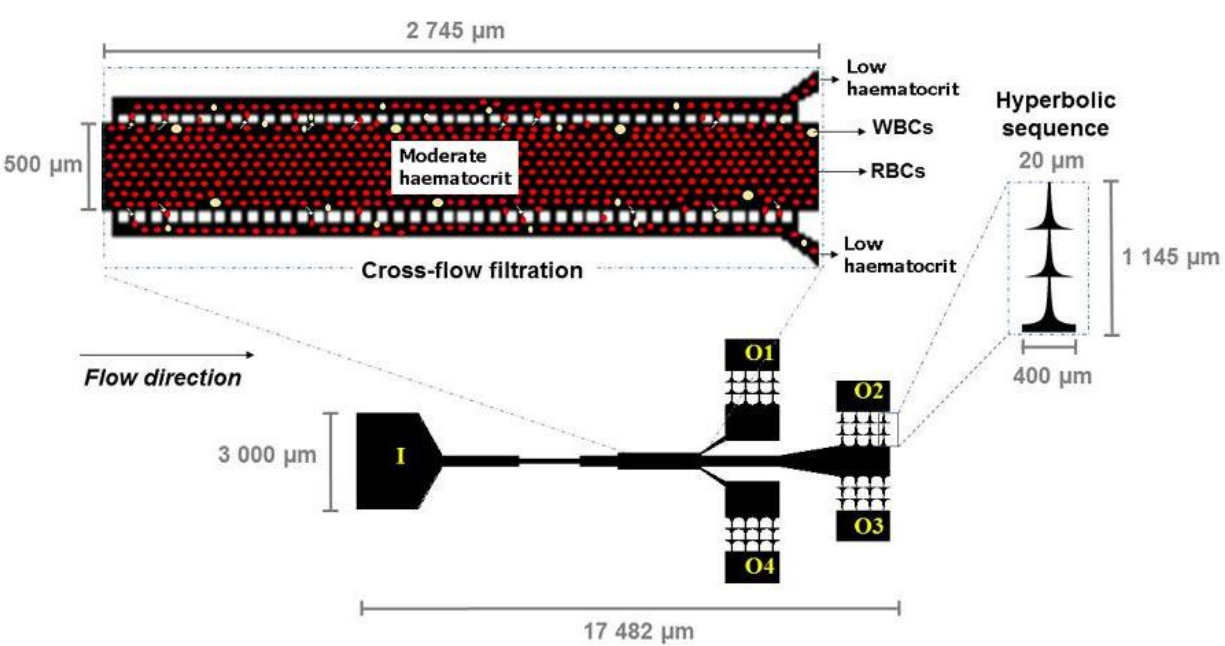

Fig. 1 Schematic view of the microfluidic device with zoom of the pillar-type filtration barrier to promote blood cells separation, as well as the hyperbolic sequence section to assess the deformation index of those blood cells. I, Inlet; O1, Outlet 1; O2, Outlet 2; O3, Outlet 3; O4, Outlet 4

hyperbolic contractions with a Hencky strain of $\sim 3$. The polydimethylsiloxane (PDMS) microchannels were fabricated using soft lithographic technique from a mask-pattern fabricated by photolitography technique using photoresistant $\mathrm{SU}-$ 8 , as previously reported by Lima et al. (2008).

\subsection{Working fluid protocol}

The working fluid used in this study was Dextran 40 (SigmaAldrich, USA) solution containing $2 \%$ of haematocrit (Hct $2 \%, v / v)$ of RBCs and equal amount of PBMCs. PBMCs were collected from whole human blood by adapting the procedure described by Sigma-Aldrich ${ }^{\text {*is }}$ (Sigma-Aldrich 2011), with the commercial solution of Histopaque-1077 (Sigma-Aldrich, USA). Briefly, venous human blood samples from healthy donors were collected into $10 \mathrm{~mL}$ BD-Vacutainers (BD, USA) tubes containing ethylenediaminetetraacetic acid (EDTA). The whole blood was divided in two equal parts in order to obtain RBCs and PBMCs with the following procedures: (i) RBCs collection, $3 \mathrm{~mL}$ of whole blood was centrifuged immediately after collection at $2500 \mathrm{rpm}$ for $10 \mathrm{~min}$ at $4{ }^{\circ} \mathrm{C}$. After removing the buffy coat and plasma, the packed RBCs were re-suspended and washed twice in physiological salt solution (PSS) with $0.9 \% \mathrm{NaCl}$ (B. Braun Medical, Germany); (ii) PBMCs collection: $3 \mathrm{~mL}$ of whole blood was carefully added to $3 \mathrm{~mL}$ of Histopaque-1077 (Sigma-Aldrich, USA) at room temperature. After centrifuged for $30 \mathrm{~min}$ at $1900 \mathrm{rpm}$, the upper layer corresponding to plasma was discarded. Therefore, the opaque interface containing PBMCs was washed three times with $6 \mathrm{~mL}$ of Hank's Balanced Salt Solution without calcium or magnesium
(HBSS, Life Technologies, USA) during $10 \mathrm{~min}$ at $1500 \mathrm{rpm}$, in order to remove possible contamination of platelets or RBCs. Then, the washed PBMCs were re-suspended in $1 \mathrm{~mL}$ of tissue culture medium, RPMI-1040 (Sigma-Aldrich, USA), and kept at $4{ }^{\circ} \mathrm{C}$ until being used in the experiments. Immediately before the experiments, the final working fluid was prepared by mixing together the collected RBCs and PBMCs in Dextran 40 solution (10\%,w/v). Dextran 40 is frequently used as substitute of the blood plasma, since it minimizes not only the sedimentation of the blood cells during the experimental assays but also cell clogging phenomenon.

\subsection{Experimental set-up}

The high-speed video microscopy system used in the present study consisted of an inverted microscope (IX71, Olympus) combined with a high-speed camera (Fastcam SA3, Photron, USA). The PDMS microchannel was placed and fixed in the microscope and the flow rate of the working fluids was kept constant at $100 \mu \mathrm{L} / \mathrm{min}$ by means of a syringe pump (PHD Ultra, Harvard Apparatus, USA) with a $5 \mathrm{~mL}$ syringe (Terumo, Japan). The fluids at the four outlets of the subchannels $(\mathrm{O} 1, \mathrm{O} 2, \mathrm{O} 3$ and $\mathrm{O} 4)$ were collected into separated Eppendorfs to be further evaluated, concerning the cell separation efficiency, as shown in Fig. 2. At the same time, the images of the flowing cells at the established flow rate were captured by the high speed camera at a frame rate of 2000 frames/s and a shutter speed ratio of $1 / 75000$, which minimized the dragging of the cells at the high flow rate in study. All the experimental assays were performed at room temperature $\left(\mathrm{T}=22 \pm 1^{\circ} \mathrm{C}\right)$. 
Fig. 2 Main experimental set-up used to perform the microfluidic tests

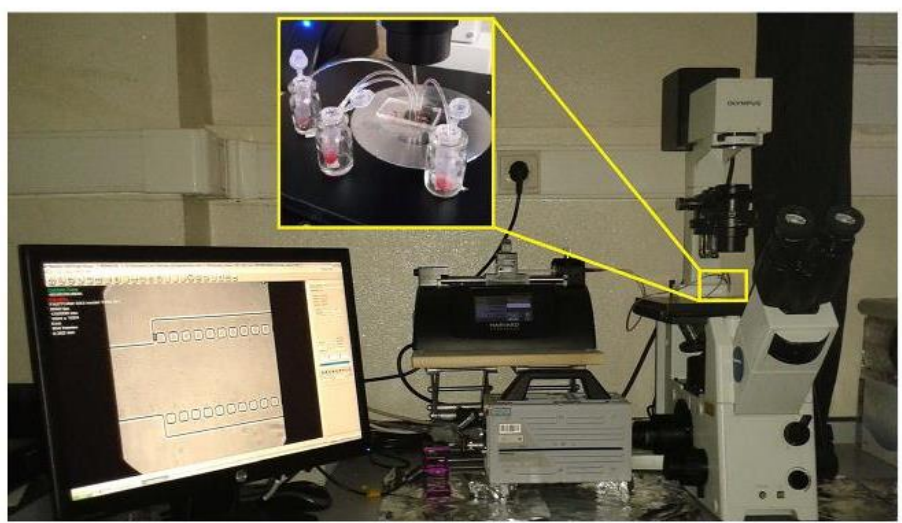

\subsection{Determination of the blood cells separation}

Individual Eppendorfs were placed close to the four outlets of the sub-channels, called as $\mathrm{O} 1, \mathrm{O} 2, \mathrm{O} 3$ and $\mathrm{O} 4$, allowing the collection, and further evaluation of the amount of blood cells that were separated by the cross-flow filtration barrier. Thus, an equal volume of $250 \mu \mathrm{L}$ from each sub-channel was treated with RBCs lysis buffer (GBiosciences, USA) in a ratio of $1: 2$ $(v / v)$, during $15 \mathrm{~min}$ at room temperature. This procedure enabled that only the RBCs were selectively lysed without affecting the viability of WBCs, and therefore, the PBMCs could be counted using a Neubauer chamber with accuracy. The method to count the PBMCs in a Neubauer chamber used in this work was the same as the recommended by Luttmann et al. (2006), where blood cells are counted within the four big squares of the Neubauer chamber. This procedure was performed in triplicate for each outlet and in two separated assays done in different days using the blood of two different healthy donors $(n=6)$.

\subsection{Image analysis}

The experimental images recorded in each test were transferred to a computer, processed and analysed by an image handling software, Image (1.46r, NIH, USA). Using this software, DI of the blood cells were calculated similarly as previously reported by Pinho et al. (2013). For all the measurements, major and minor axis lengths of the blood cells were used to determine RBCs and PBMCs DIs. The formula used to calculate the DI is presented as Eq. (1):

$\mathrm{DI}=\frac{(\text { Lmajor }- \text { Lminor })}{(\text { Lmajor }+ \text { Lminor })}$

where, $\mathrm{L}_{\text {major }}$ and $\mathrm{L}_{\text {minor }}$ refer to major and minor axis lengths of the blood cell, respectively.
For the assessment of the DI of each blood cells, 92 individual cells of RBCs and 76 PBMCs were randomly measured in the centre line position along the hyperbolic channel. Hence, a total of 168 individual blood cells were assessed to calculate the DI.

\section{Results and discussion}

\subsection{Separation of blood cells in the microfluidic device}

The main goal of this study is the development of a simple and integrative microfluidic device capable to perform at once and in continuous flow, the separation of a certain amount of blood cells from an initial in vitro blood sample, and simultaneously use the reduced amount of blood cells to perform their deformability assessment. Hence, this device can be a useful microfluidic tool, capable to assess and study the blood cells DIs, as they can be used as label-free biomarkers for the assessment of blood diseases. To gain insight in the deformability behavior of leukocyte cells, we have decided to investigate the PBMCs (i.e., lymphocyte, monocyte or macrophage), which are main actors in the inflammatory processes and linked to many blood diseases.

To achieve the best flow conditions to perform the microfluidic experimental assays, several experimental parameters had to be previously studied. One of the most critical parameter in all microfluidic tests is the choice of the most suitable flow rate that better fits on the purpose of the microfluidic study. Therefore, in order to evaluate the optimal flow rate for the better cell separation efficiency, a series of trial assays were previously made in the range between 10 and $200 \mu \mathrm{l} / \mathrm{min}$. In these screening experiments, the blood cells (PBMCs and RBCs) were tracked at three representative positions (middle of the main channel, just above the pillars-wall and at the region between the middle position and pillars-wall)

\section{Q Springer}


at the cross-flow filtration region of the microchannel device (data not shown). To assess this data the manual tracking "MtrackJ" plugin (Meijering et al. 2006) from the image handling software, ImageJ (1.46r, NIH) was used to track PBMCs and RBCs, as similarly reported by Leble et al. (2011) and shown in Fig. 3 (more details in the supplementary video 1). Figure 3 shows clearly that the pillars provide a barrier to WBCs as a PBMC is rolling along the cross-flow pillars promoting this cell to flow within the main channel. In contrast, RBCs tend to deform and pass through the pillars into the branch channel.

As a result, the flow rate $100 \mu 1 / \mathrm{min}$ was considered to be the most suitable to perform this study, due to the maintained flow stability, absence of clogging or jamming of the cells, as well as a better separation of the tracked blood cells along the cross-flow filtration channel. Therefore, this optimized flow rate allowed that just a small concentration of blood cells flow through the cross-flow pillar barrier, which promoted the optimum conditions to assess the individual cells DI. In contrast, the lower flow rates used in the screening experiments $(10,25$ and $50 \mu \mathrm{l} / \mathrm{min})$, often led to severe clogging and jamming of the blood cells. In particular, the PBMCs were often trapped within the pillars barrier with these lower flow rates, restricting the continuity of these assays and consequently compromising the assessment of the cells DIs. A possible explanation for these observed clogging and jamming phenomena is that at lower flow rates, stagnation regions where the cells velocity are close to zero, are more likely to happen around the crossflow pillars. On the other hand, tests with higher flow rate, such as $200 \mu \mathrm{l} / \mathrm{min}$, have resulted in high internal pressure and leakage of the working fluid. Note that high flow rates also increase the complexity of controlling the working fluid within the microchannel and measuring the deformability of the cells flowing through the hyperbolic contractions. Thus, according to these screening results, it was decided to select the flow rate of $100 \mu \mathrm{l} / \mathrm{min}$, as the most suitable to perform our experimental tests.

Table 1 shows the amount of PBMCs cells $/ \mathrm{mL}$ counted by means of a Neubauer chamber, as previously described in section 2.4., after the RBCs lysis treatment be applied into all the fluid samples collected at the four outlets.

The results shown in Table 1 demonstrate that even with a very simple cross-flow filtration geometry (consisting in just one linear sequence of square pillars), it is possible to obtain a desired amount of PBMCs for the further assessment of cells DI, which in this case represents about $25 \%$ of cells from the initial working fluid. Figure 4 shows a representative illustration of the separation strategy and blood cells flowing through the pillar cross-flow barrier into the four outlets.

As it can be seen in Fig. 4, the combination of the crossflow filtration with an optimal flow rate generates a cell-free layer in the lateral outlets promotes a decreasing on the total amount of blood cells flowing through that outlets. These decreasing of PBMCs in $\mathrm{O} 1$ and $\mathrm{O} 4$ were also qualitatively observed in the amount of RBCs. However, the qualitative data of the collected RBCs into these outlets had to be sacrificed in order to improve the accuracy of the counted PBMCs within the Neubauer chamber. As a result, the RBCs were lysed as previously described in the section 2.4., with minimal cellular effect on the WBCs.

\subsection{Blood cells deformation assessment}

In literature there are several published works regarding the correlation between RBCs, abnormal deformability and some diseases (e.g., malaria (Suwanarusk et al. 2004), diabetes (Sabo et al. 1993), coronary diseases (Yaylali et al. 2013), among others), showing the interest of this topic by several research groups. However, even though WBCs are known to be important cells, as they defend our body against bacteria, virus and other harmful agents, there are few works published in literature concerning the in vitro assessment of their deformability, motion and interaction with other blood cells in microfluidic devices. Therefore, in this study we measured the DI of both RBCs and WBCs (i.e., PBMCs) flowing through hyperbolic microchannels located downstream the cross-flow microfilters, designated as $\mathrm{O} 1$ and $\mathrm{O} 4$ (cf. Fig. 1). As described above, the cross-flow filtration used in this study has promoted the reduction of the concentration of both
Fig. 3 Trajectories of both RBC and $\mathrm{PBMC}$ flowing around the pillars. The PBMC rolls along the pillars in the direction of primary flow whereas the RBC deforms and pass through the narrow gap between the pillars into the branch channel (see also supplementary video 1)

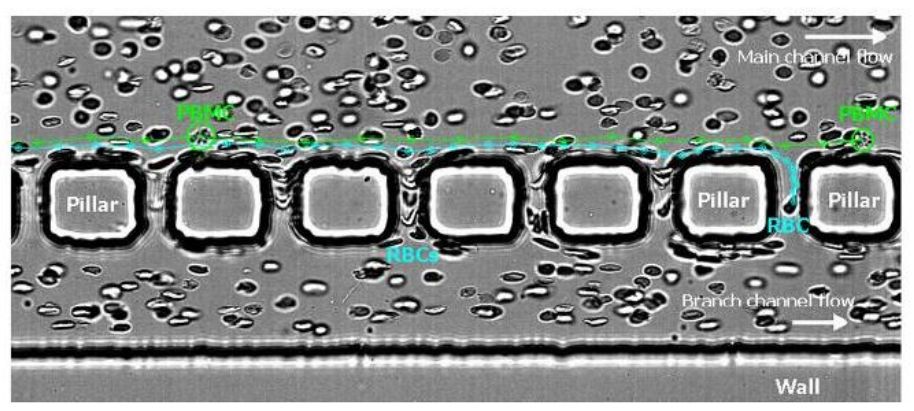


Table 1 Separation results of the PBMCs in continuous flow rate at $100 \mu \mathrm{L} /$ min using a simple cross-flow filtration microdevice

\begin{tabular}{lll}
\hline Microdevice section & PBMCs \pm SD $\left(10^{4}\right.$ cells $\left./ \mathrm{mL}\right)$ & PBMCs (\%) \\
\hline Outlet 1 & $19 \pm 4.8$ & 22 \\
Outlet 2,3 & $46 \pm 7.9$ & 52 \\
Outlet 4 & $22 \pm 6.0$ & 26 \\
\hline
\end{tabular}

Results are presented as Mean \pm Standard Deviation $\left(10^{4}\right.$ cells $\left./ \mathrm{mL}\right)$ and in percentage $(\%)(n=6)$

PBMC Peripheral blood mononuclear cells

WBCs and RBCs passing to the outlets $\mathrm{O} 1$ and $\mathrm{O} 4$ (as shown in Table 1 and Fig. 4). This low concentration was appropriate to obtain video images with single individual blood cells. However, this is not the case of the hyperbolic channels located around the outlets $\mathrm{O} 2$ and $\mathrm{O} 3$, where the high concentration of cells does not allow to accurately perform deformability assessment of individual blood cells.

After the cells concentration be decreased from the initial in vitro blood sample, due to the cross-flow filtration, the initial flow rate was slightly reduced with the presence of reservoirs located immediately before of each hyperbolic sequences in the four outlets of the microfluidic device (cf. Fig. 1). These geometries allowed that the DI assessment of the blood cells could be made by means of our high speed video camera, with minimal dragging of the cells.

Figure 5 shows the DIs of the blood cells (RBCs and PBMCs) flowing along the axial position of a hyperbolic channel with a sudden expansion, as well as the average or pseudo shear rate, $\bar{\gamma}=\frac{U}{D_{h}}$, at each hyperbolic region, where $U$ is the mean velocity of the blood cells obtained at each region and $D_{h}$ is the hydraulic diameter of each microchannel section.

Overall, the DI of both cells has a maximum value at the region right before the exit of the contraction, where the average shear rate is also the highest $\left(1761.6 \mathrm{~s}^{-1}\right)$. In fact, the maximum average shear rate obtained at the narrowest region is in good agreement with the shear rate values encountered in arterioles $\left(\sim 1600 \mathrm{~s} \mathrm{~s}^{-1}\right)$, which have dimensions similar to the hyperbolic contractions used in this work $(\sim 20 \mu \mathrm{m})$ (Papaioannou and Stefanadis 2005). After the narrowest section, cells tend to recover to its initial shape that corresponds to a minimal value of the DI, where cells are no longer exposed to extensional flow and the average shear rate reduces to extremely low value $\left(60.7 \mathrm{~s}^{-1}\right)$, similarly to the values encountered at large veins. Moreover, the shear stress encountered in the arterioles (shear rate $\sim 1600 \mathrm{~s}^{-1}$ ) is around $55 \mathrm{dyn} / \mathrm{cm}^{2}$ and this value decreases to $5 \mathrm{dyn} / \mathrm{cm}^{2}$ at large veins, where the shear rate is $\sim 100 \mathrm{~s}^{-1}$ (Papaioannou and Stefanadis 2005). Therefore, our results show that both shear and extensional flows generated by the hyperbolic channels promote cell response to shear stress in a similar way that happens in in vivo environments.

Figure 5 also shows the difference between the DI obtained by the RBCs and PBMCs when exposed to the same strong extensional flow and shear stress conditions. It should be noted that the hyperbolic contraction geometry to measure the cells deformability with a Hencky strain of $\sim 3$ was chosen mainly due to the strong extensional flow generated in the middle of the microchannel, which is dominant over the shear flow. To better understand the influence of the extensional flow and shear stress on the deformability of both RBCs and PBMCs, average DI values at the two key regions of the microchannel (narrow and recuperation section) were selected and defined as section 1 and 2 (Fig. 6).

The first DI measurement was made in the hyperbolic constriction section (section 1) that promotes the elongation of the cells caused by the strong extensional and shear flow (Lee et al. 2009; Yaginuma et al. 2013). The second DI assessment was measured in the recovery section caused by the sudden expansion (section 2), where the recovery to the normal size and shape of the blood cells can be seen. These two DIs assessment provided a better understanding of the deformation phenomenon by showing the different behaviour that blood cells have when subjected to the same strong extensional flow and high shear rate (section 1) and then the recovery of their initial shape, which
Fig. 4 Blood cells flowing through cross-flow filtration barrier to the direction of the outlets and hyperbolic chamber for their further DI assessment

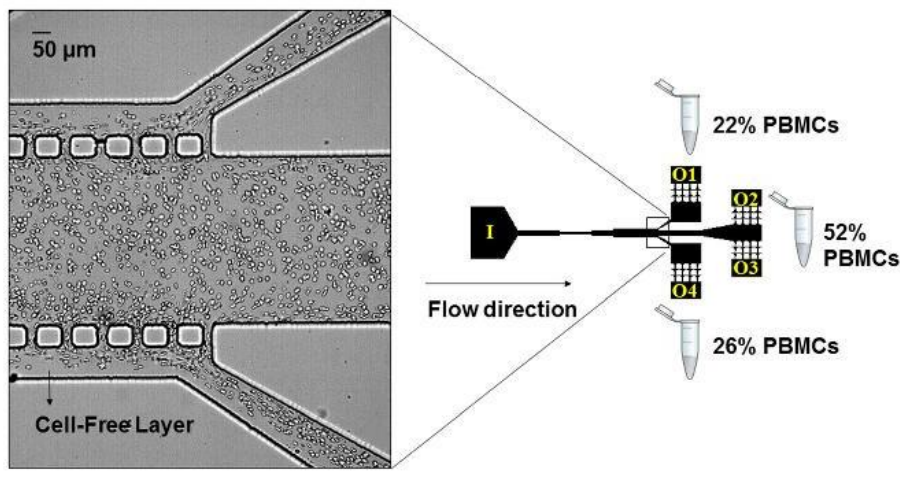

\section{Dspringer}



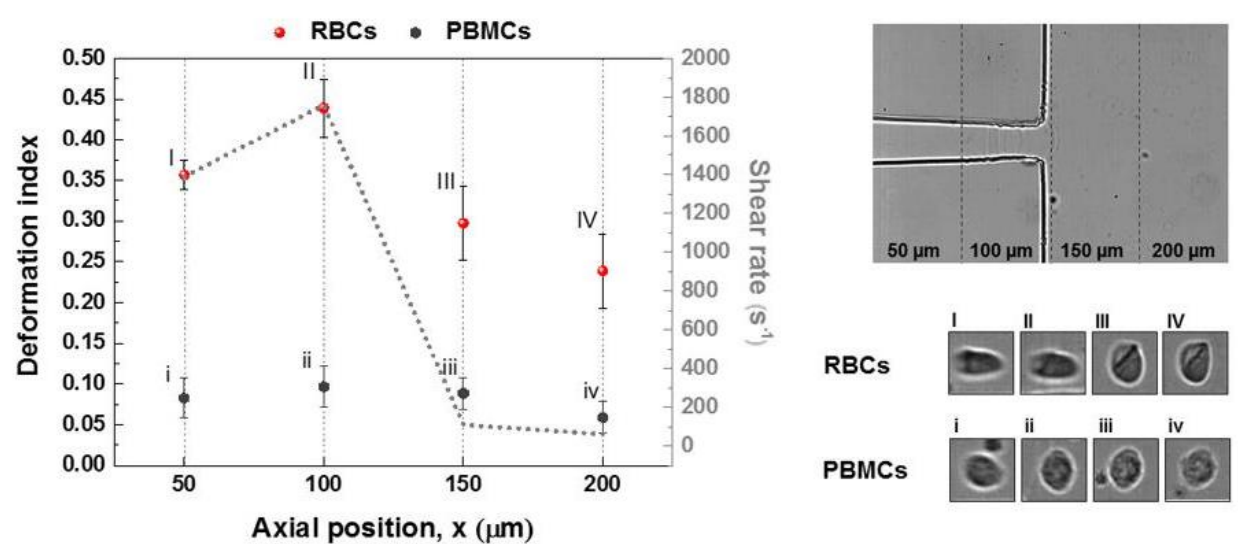

Fig. 5 Deformation index of blood cell (RBCs and PBMCs) flowing along the axial position of the hyperbolic channel followed by a sudden expansion (red and black dots), as well as the average shear rate at each

hyperbolic region (dotted line). Error bars shows a $95 \%$ of confidence interval ( $n=168$ blood cells)

corresponds to a minimal value of the DI, where cells are no longer exposed to these deformable hemodynamic conditions (section 2). The results show that PBMCs, when submitted to a strong extensional flow and high flow rate, can reach a maximum DI of 0.10 , followed by a small decrease of the DI to about 0.06 , in the recovery section $(n=76)$. It is worth mentioning that similar results were obtained by Gossett et al. (2012), regarding the deformation of untreated PBMCs subjected to a stretching extensional flow in a microfluidic deformability cytometer. By calculating the DI in the same way that Gossett et al. (2012) (length of the longer axis divided by the perpendicular of the shorter axis), our results shown a mean deformation of PBMCs about $1.21 \pm 0.13(n=76)$, which is in good agreement with the data obtained by Gossett et al. (2012) where the mean deformation of these blood cells were calculated to be $1.18(n=377)$. These results reveal that PBMCs under the applied in vitro conditions tend to behave as a non-deformed cell, indicating the importance of the pro-inflammatory mediators (cytokines, chemokines or bacterial peptides) have into the deformation of these cells, as observed in in vivo (Liu and Wang 2004) for the active WBCs deformation. Additionally, the results show the importance of the leukocyte-endothelial cell interaction, which is intimately linked to the cytoskeleton structures of the WBCs (namely the microvilli projections of them) and the nucleus content (e.g., cytoplasm viscosity). In fact, the cytoskeleton structure and internal composition of the WBCs are the main reasons for the rolling and arrest (firm adhesion) phenomena that allows their capture from the blood flow by the endothelial cells and further migration of these blood cells to search for bacteria or fungi through the body (Khismatullin 2009). These phenomena (specially the rolling) were also observed in this study along the whole microfluidic device (either in the cross-flow pillars, as shown in Fig. 3 and supplementary video 1, and in the hyperbolic channels), which we believe is the main reason for the difference of blood cells contents (WBCs and RBCs) that we have observed in the four outlets, as well as for the lower deformability observed in the hyperbolic channel, when compared with the RBCs DIs. Moreover, Fig. 6 shows that the RBCs, when submitted to the same strong extensional flow and high shear rate, tend to elongate (especially when they travel along the centreline of the hyperbolic section), reaching a maximum DI of $0.44 \pm 0.04$, which corresponds fourfold higher than the deformability reached by PBMCs. Also, it can be observed in this figure that this high RBC deformability is followed by a dramatic decrease of their DI to $0.24 \pm 0.05$. This latter result corroborate with the ones obtained by Lee et al. (2009) and Yaginuma et al. (2013), where they found that RBCs change

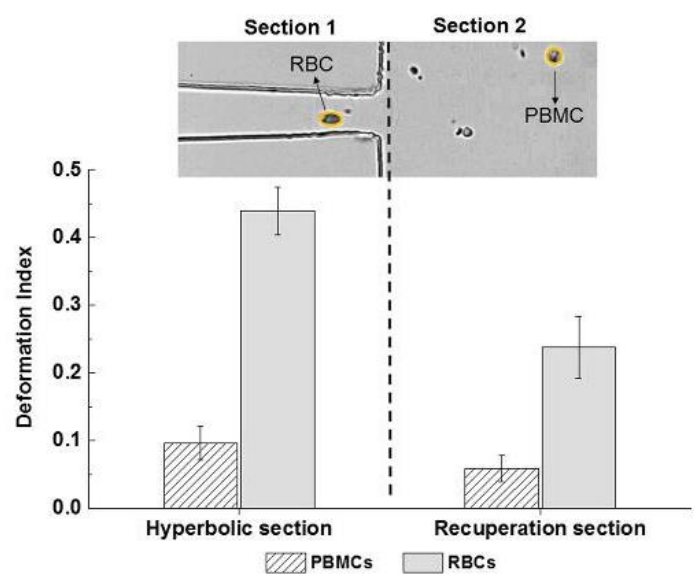

Fig. 6 Averaged deformation index obtained from PBMCs and RBCs flowing within the hyperbolic and recuperation section of the microchannel device. Error bars shows a $95 \%$ of confidence interval $(n=168)$ 
from a nearly circular to an elliptical shape and become increasingly elongated as they flow through a hyperbolic contraction. Hence, the results obtained by this study indicate that RBCs under in vitro and passive hemodynamic conditions, presents higher deformability behaviour when compared to the PBMCs deformability results. Since the WBCs are naturally known as deformable cells in their biological environment, this paradoxical in vitro results corroborated the fact that Gossett et al. (2012) pointed to the relevance of pro-inflammatory mediators in the activation of the deformation behaviour of these blood cells, as well as for the importance to study the mechanical properties of each type of WBCs when subjected to passive deformability. Additionally, these in vitro hemodynamic behaviours encountered among different blood cells may have the potential to become a new $\mu$ TAS able to use these findings to improve the separation of different blood cells from the whole blood based on their natural and passive hydrodynamic behaviour, and therefore, eventually, allowing the development of a simple and disposable clinical microfluidic system.

\section{Conclusions}

In this work we have successfully designed, fabricated and tested a novel microfluidic device able to perform continuously and simultaneously both separation of a certain amount of blood cells from an initial blood sample, as well as to assess the deformability of them, by using respectively, cross-flow microfilters and hyperbolic microchannels. The cross-flow filtration strategy has shown the ability to separate from the initial in vitro blood sample, approximately $25 \%$ of PBMCs and to collect directly to the two outlets located downstream of this barrier. This separation efficiency was proven to be the most adequate way to assess blood cells deformability by using our high-speed video microscopy system. The major advantage of this separation technique was the ability to investigate, for the first time, the effect of extensionaldominated flows on the deformability of both PBMCs and RBCs under the same in vitro conditions using hyperbolic chambers. The DI results have shown that PBMCs have a non-deformable behaviour when subjected to passive hemodynamic conditions even with a strong extensional flows, which is in good agreement with the recently published study performed by Gossett et al. (2012). In contrast, RBCs have shown to have a much higher deformable behaviour when subjected to the same in vitro conditions (fourfold higher than the deformability reached by PBMCs). Although there are many advantages with the proposed microfluidic device, including its low cost, ease of fabrication and cell separation simplicity, its biggest challenges are its ability to perform whole blood cells separation without any problems of clogging or jamming and the need to use a high-speed video microscopy system to measure the cells deformability. Further improvements in the proposed microfluidic design and the integration of optical components within the device are expected to lead to the creation of a novel $\mu \mathrm{TAS}$ for routine cell screening in clinical and research applications.

Acknowledgments The authors acknowledge the financial support provided by PTDC/SAU-ENB/116929/2010 and EXPL/EMS-SIS 2215/2013 from FCT (Fundação para a Ciência e a Tecnologia), COMPETE, QREN and European Union (FEDER). R. O. Rodrigues, D. Pinho and $\mathrm{V}$. Faustino acknowledge respectively, the $\mathrm{PhD}$ scholarships SFRH/BD/97658/2013, SFRH/BD/89077/2012 and SFRH/BD/99696/ 2014 granted by FCT. The authors would also like to thank Dr. Ângela Fernandes for providing the blood samples and Dr. Ricardo Calhelha for supplying the tissue culture medium used in this work.

\section{References}

G.C. Agbangla, É. Climent, P. Bacchin, Sep. Purif. Technol. 101, 42-48 (2012)

E.L. Bradley, L. Bernard, P. Thomas, J. Brian, J. Micromech. Microeng. 22, 025009 (2012)

X. Chen, D. Cui, C. Liu, H. Li, J. Chen, Anal. Chim. Acta 584, 237-243 (2007)

X. Chen, D.F. Cui, C.C. Liu, H. Li, Sens. Actuators B Chem. 130, $216-$ 221 (2008)

R. Covar, M. Gleason, B. Macomber, L. Stewart, P. Szefler, K. Engelhardt, J. Murphy, A. Liu, S. Wood, S. DeMichele, E.W. Gelfand, S.J. Szefler, Clin. Exp. Allergy 40, 1163-1174 (2010)

V. Faustino, D. Pinho, T. Yaginuma, R. Calhelha, I.F.R. Ferreira, R. Lima, BioChip J. 8, 42-47 (2014)

A.E. Frampton, C.E. Fletcher, T.M. Gall, L. Castellano, C.L. Bevan, J. Stebbing, J. Krell, Expert. Rev. Mol. Diagn. 13, 425-430 (2013)

J. Fu, B.E. Sha, L.L. Thomas, J. Acquir. Immune Defic. Syndr. 56, 16-25 (2011)

K. Georgieva, D.J. Dijkstra, H. Fricke, N. Willenbacher, J. Colloid Interface Sci. 352, 265-277 (2010)

D.R. Gossett, W.M. Weaver, A.J. Mach, S.C. Hur, H.T. Tse, W. Lee, H. Amini, D. Di Carlo, Anal. Bioanal. Chem. 397, 3249-3267 (2010)

D.R. Gossett, H.T.K. Tse, S.A. Lee, Y. Ying, A.G. Lindgren, O.O. Yang, J. Rao, A.T. Clark, D. Di Carlo, Proc. Natl. Acad. Sci. U. S. A. 109, 7630-7635 (2012)

H.W. Hou, A.A. Bhagat, A.G. Chong, P. Mao, K.S. Tan, J. Han, C.T. Lim, Lab Chip 10, 2605-2613 (2010)

H.M. Ji, V. Samper, Y. Chen, C.K. Heng, T.M. Lim, L. Yobas, Biomed. Microdevices 10, 251-257 (2008)

Z. Jinlong, G. Qiuquan, L. Mei, Y. Jun, J. Micromech. Microeng. 18 125025 (2008)

D.B. Khismatullin, in Leukocyte rolling and adhesion: Current topics in membranes, ed. by K. Ley, vol. 64 (Academic, New York, 2009), pp. $47-111$

M. Kim, S. Mo Jung, K.H. Lee, Y. Jun Kang, S. Yang, Artif. Organs 34, 996-1002 (2010)

V. Leble, R. Lima, R. Dias, C. Fernandes, T. Ishikawa, Y. Imai, T. Yamaguchi, Biomicrofluidics 5, 044120-044120-044115 (2011)

S. Lee, Y. Yim, K. Ahn, S. Lee, Biomed. Microdevices 11, 1021-1027 (2009)

R. Lima, S. Wada, S. Tanaka, M. Takeda, T. Ishikawa, K. Tsubota, Y. Imai, T. Yamaguchi, Biomed. Microdevices 10, 153-167 (2008)

X.H. Liu, X. Wang, J. Biomech. 37, 1079-1085 (2004)

W. Luttmann, K. Bratke, M. Kupper, D. Myrtek, Immunology, vol. 1 (Elsevier, Philadelphia, 2006)

E. Maes, B. Landuyt, I. Mertens, L. Schoofs, PLoS One 8, e61933 (2013) 
E. Meijering, I. Smal, G. Danuser, IEEE Signal Process. Mag. 23, 46-53 (2006)

S. Metz, C. Trautmann, A. Bertsch, R. Ph, J. Micromech. Microeng. 14, 324 (2004)

S.K. Murthy, P. Sethu, G. Vunjak-Novakovic, M. Toner, M. Radisic, Biomed. Microdevices 8, 231-237 (2006)

E. Ortega, R. Gilabert, I. Nuñez, M. Cofán, A. Sala-Vila, E. de Groot, E. Ros, Atherosclerosis 221, 275-281 (2012)

T.G. Papaioannou, C. Stefanadis, Hellenic J. Cardiol. 46, 9-15 (2005)

D. Pinho, T. Yaginuma, R. Lima, BioChip J. 7, 367-374 (2013)

A. Sabo, V. Jakovljevic, M. Stanulovic, L. Lepsanovic, D. Pejin, Int. J. Clin. Pharmacol. Ther. Toxicol. 31, 1-5 (1993)

S.S. Shevkoplyas, T. Yoshida, L.L. Munn, M.W. Bitensky, Anal. Chem. 77, 933-937 (2005)

Sigma-Aldrich, Histopaque-1077: product information. St. Louis, MO, USA, (2011)
R. Suwanarusk, B.M. Cooke, A.M. Dondorp, K. Silamut, J. Sattabongkot, N.J. White, R. Udomsangpetch, J. Infect. Dis. 189, 190-194 (2004)

K. Tae Goo, Y. Yong-Jin, J. Hongmiao, L. Pei Yi, C. Yu, J. Micromech. Microeng. 24, 087001 (2014)

M. Tanino, R. Matoba, S. Nakamura, H. Kameda, K. Amano, T. Okayama, H. Nagasawa, K. Suzuki, K. Matsubara, T. Takeuchi, Biochem. Biophys. Res. Commun. 387, 261-265 (2009)

V. VanDelinder, A. Groisman, Anal. Chem. 78, 3765-3771 (2006)

V. VanDelinder, A. Groisman, Anal. Chem. 79, 2023-2030 (2007)

T. Yaginuma, M.S.N. Oliveira, R. Lima, T. Ishikawa, T. Yamaguchi, Biomicrofluidics 7, 054110 (2013)

X. Yang, J.M. Yang, Y.-C. Tai, C.-M. Ho, Sensors Actuators A Phys. 73, 184-191 (1999)

Y.T. Yaylali, I. Susam, E. Demir, M. Bor-Kucukatay, B. Uludag, E. KilicToprak, G. Erken, D. Dursunoglu, J. Coron. Artery Dis. 24, 11-15 (2013) 


\title{
C. Polymer microfluidic devices: an overview of fabrication methods
}

\section{Polymer microfluidic devices: an overview of fabrication methods}

\begin{abstract}
Raquel O. Rodrigues ${ }^{1}$, Rui Lima ${ }^{2}$, Helder T. Gomes ${ }^{3}$, Adrián M. T. Silva ${ }^{4}$ ${ }^{1}$ LCM - Laboratory of Catalysis and Materials - Associate Laboratory LSRE-LCM, Faculdade de Engenharia da Universidade do Porto, Porto, Portugal / Polytechnic Institute of Bragança, Bragança, Portugal (raquel.rodrigues@ipb.pt); ${ }^{2}$ University of Minho, Mechanical Engineering Department, Guimarães, Portugal / CEFT, Faculdade de Engenharia da Universidade do Porto, Porto, Portugal / Polytechnic Institute of Bragança, Bragança, Portugal (ruimec@ipb.pt); ${ }^{3}$ LCM - Laboratory of Catalysis and Materials - Associate Laboratory LSRE-LCM, Faculdade de Engenharia da Universidade do Porto, Porto, Portugal / Polytechnic Institute of Bragança, Bragança, Portugal (htgomes@ipb.pt); 4 LCM - Laboratory of Catalysis and Materials - Associate Laboratory LSRE-LCM, Faculdade de Engenharia da Universidade do Porto, Porto, Portugal (adrian@fe.up.pt)
\end{abstract}

\begin{abstract}
The amount of applications associated with microfluidic devices is increasing since the introduction of Lab-on-a-chip devices in the 1990s, especially regarding biomedical and clinical fields. However, in order for this technology to leave the fundamental research and become a day-life technology (e.g., as point-of-care testing), it needs to be disposable and reasonably less expensive. Polymers, due to their several advantages, such as easier microfabrication and low-cost, fill these needs. Several methods are reported regarding microfabrication and, thus, the main aim of the present work is to provide an overview of the most relevant microfabrication techniques found in literature employing polymers, clarifying also the main advantages and disadvantages of each technique and especially considering their cost and time-consumption. Moreover, a future outlook of lowcost microfabrication techniques and standard methods is provided.
\end{abstract}

Subject Headings. Nanotechnology, Medical diagnosis, Hand tool.

Author Keywords. Microfabrication, PDMS, Lab-on-a-chip, Low-cost techniques.

\section{Introduction}

Microfluidic devices (Figure 1) can be defined as the set of technologies which handles and processes small fluid volumes (e.g., $\mu \mathrm{L}, \mathrm{nL}$, and $\mathrm{pL}$ ) through microchannels geometries, with dimension of tens to hundreds of micrometers, embedded in a chip (Halldorsson et al. 2015, Monošík and Angnes 2015, Sia and Whitesides 2003, Whitesides 2006).

Related with these characteristics, since small amount of reagents and samples are used, microfluidic devices are suitable for analytical purposes with other several advantages, such as short time for analysis, reduction of reagent costs, low fabrication cost, miniaturization, sensitivity, selectivity, repeatability, portability and biocompatibility (Monošík and Angnes 2015, Whitesides 2006). Furthermore, microfluidic devices can be used as an integrative multiple processes device, called as Lab-on-a-chip (LOC) or micro total analysis system ( $\mu$ TAS). This concept of "miniaturized total chemical analysis system", known in our days as $\mu$ TAS or LOC, was introduced by Andreas Manz and co-workers in 1990 (Manz, Graber and Widmer 1990), and since then, the scientific expectations on this technology and analytical possibilities have increased as analytical tool (Whitesides 2006) and also a tool capable to improve the global health (van Reenen et al. 2014, Yager et al. 2006). Some application examples at the 
Polymer microfluidic devices: An overview of fabrication methods Raquel O. Rodrigues, Rui Lima, Helder T. Gomes, Adrián M. T. Silva

laboratory scale are found in clinical diagnostics, as point-of-care testing (Do et al. 2008, Liu et al. 2014, Novo, Chu and Conde 2014), but also in environment monitoring (Jeong et al. 2014, Mehta et al. 2006, Sun et al. 2014), food industry (Fronczek, You and Yoon 2013, Zhang, Zuo and Ye), microelectronics (Catalano et al. 2014, Daikuzono et al. 2015) and in numerous biochemical and biological processes, such as analysis of blood samples (Lima et al. 2008), drug screening (Nason et al. 2011), cell counting and sorting (Pratt et al. 2011), cell culture studies (Shi, Liu and Chen 2011), polymerase chain reaction (PCR) (Pan et al. 2010), DNA sequencing (Paegel, Blazej and Mathies 2003), among many others.

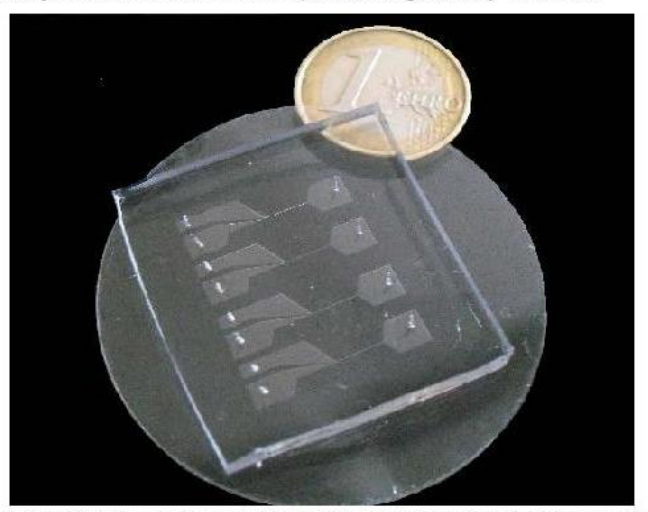

Figure 1. Microfluidic device designed for partial blood cells separation and deformability assessment.

However, to achieve future mass-market commercialization, it is crucial to find fabrication methods that allow their low cost production in large scale. This concern is especially important for medical applications, where the microdevices should be disposable, to avoid cross contamination or to be used in point-of-care testing (Attia, Marson and Alcock 2009).

Polymers, due to their several advantages (e.g., low-cost material, easier microfabrication over the other materials such as glass and silicon) are the most promising materials to fill the needs of mass-market utilization.

Due to the importance of the microfluidic field and the variety of microfabrication methods reported in literature, the present work intends to provide an overview of the most relevant techniques that use polymers, and to present recent low-cost techniques, that are of particular interest in research.

\section{Microfabrication techniques}

The first microfluidic device dates from 1979, when miniaturization of a gas chromatograph was developed at the Stanford University (Terry, Jerman and Angell 1979).

Similar to this first microfluidic device, most of the early systems were fabricated by microelectromechanical systems (MEMS) technology, due to the available semiconductor industry, highly developed in that time. Thus, techniques such as photolithography, thin film metallization and chemical etching on silicon and glass, were applied for the fabrication of these new type of devices (McDonald et al. 2000, Wu and Gu 2011).

Later, glass materials started to gain more interest, mainly due to the biocompatibility for applications in the biomedical field. However, the microfabrication difficulties in these glass materials, allied to the need of a cleanroom environment, high temperature requirement for sealing and expensive cost of glass materials, have hampered their wider application in microfluidics (Wu and Gu 2011). Due to these issues, a tremendous effort has been made since 
then, in order to find alternative materials that allow a more cost-effective production and easier microfabrication.

Polymers, due to its characteristics, fill the above needs and bring other advantages, such as good optical transparency, biocompatibility, chemical and mechanical properties as well as friendly system integration (e.g., interconnection with inlets/outlets) (Wu and Gu 2011). Therefore, these materials enable a high volume of production with good reproduction, lower cost of fabrication and also versatility in the design. These properties are of utmost importance to the creation of disposable microfluidic chips for biomedical and clinical applications. Nevertheless, polymers have some limitations regarding their properties or processing techniques in comparison to glass, such as limited operation-temperature range, higher autofluorescence and limited surface modification techniques (Attia, Marson and Alcock 2009).

For a better perception of the main characteristics of polymers and glass, Table 1 shows a comparison of properties that are important for the fabrication of microfluidic devices and to the selection of the microdevice substrate.

Properties

Polymers

Manufacturing cost
$\begin{aligned} & \text { Complexity of } \\ & \text { Fabrication }\end{aligned}$
$\begin{aligned} & \text { Operation } \\ & \text { temperature }\end{aligned}$
Optical properties
and detection of
fluorescence
Bonding

Compatibility with organic solvents, strong acids and bases

Gas permeability

Design

\section{Limited temperatures range due to relatively low $T_{g}$ (glass transition temperature) compared to glass.}

In general, lower optical transparency and higher autofluorescence compared to glass.

Many bonding options are available, such as adhesives, thermal fusion, ultrasonic welding and mechanical clamping.

In general polymers are not resistant to most organic solvents or strong acids or bases.

High gas permeability.

Polymer fabrication techniques are more flexible to complex geometries, such as different cross-sections, heights and higher aspect ratio square channels.

\section{Glass}

Higher manufacturing cost related to substrates and cleanroom facilities.

Fabrication steps are more time consuming and expensive. Wet chemistry is used.

Wide range of working temperature compared to polymers.

Excellent optical properties.

Bonding options are more time consuming than polymers and include thermal, adhesive and anodic bonding.

Good resistance to organic solvents and acids.

Absence of gas permeability which is required for some biological and cell culture applications.

Limited to simple designs (2D) due to the nature of the etching process.

Table 1. Comparison of properties between polymers and glass for the fabrication of microfluidic devices. Adapted from (Attia, Marson and Alcock 2009)

The most popular polymers used to fabricate microfluidic devices are poly(methyl methacrylate) (PMMA), cyclic olefin copolymer (COC), poly(styrene) (PS), poly(carbonate) (PC), poly(ethyleneterephthalate glycol) (PETG) and poly(dimethylsiloxane) (PDMS) (Becker and Locascio 2002, Fiorini and Chiu 2005, Li et al. 2008, Sollier et al. 2011, Wu and Gu 2011). Table 2 shows a comparison of the main properties between these polymers and the typical fabrication techniques that are applied. 
Polymer microfluidic devices: An overview of fabrication methods Raquel O. Rodrigues, Rui Lima, Helder T. Gomes, Adrián M. T. Silva

\begin{tabular}{|c|c|c|c|}
\hline Polymer & Main characteristics & Fabrication techniques & References \\
\hline PMMA & $\begin{array}{l}\text { Thermoplastic. Transparent. UV } \\
\text { resistance. Low water absorption. } \\
\text { Good abrasion resistance. }\end{array}$ & $\begin{array}{l}\text { Injection moulding. Hot } \\
\text { embossing. Laser } \\
\text { photoablation. X-ray } \\
\text { lithography. }\end{array}$ & $\begin{array}{c}\text { (Attia, Marson and Alcock } \\
\text { 2009, Becker and Locascio } \\
\text { 2002, Fiorini and Chiu } \\
\text { 2005, Li et al. 2008) }\end{array}$ \\
\hline $\mathrm{COC}$ & $\begin{array}{l}\text { Thermoplastic with high } \\
\text { transparency. High heat } \\
\text { resistance. Low water absorption. } \\
\text { High stiffness and strength. }\end{array}$ & $\begin{array}{l}\text { Injection moulding. } \\
\text { Hot embossing. }\end{array}$ & $\begin{array}{c}\text { (Attia, Marson and Alcock } \\
\text { 2009, Fiorini and Chiu } \\
\text { 2005, Khanarian and } \\
\text { Celanese 2001, Li et al. } \\
\text { 2008) }\end{array}$ \\
\hline PS & $\begin{array}{l}\text { Thermoplastic. Excellent } \\
\text { electrical properties. Resistant to } \\
\text { a wide variety of chemicals. }\end{array}$ & $\begin{array}{l}\text { Injection moulding. } \\
\text { Hot embossing. Laser } \\
\text { photoablation. }\end{array}$ & $\begin{array}{l}\text { (Becker and Locascio } \\
\text { 2002, Fiorini and Chiu } \\
\text { 2005, Li et al. 2008) }\end{array}$ \\
\hline PC & $\begin{array}{l}\text { Transparent thermoplastic. High } \\
\text { heat resistance. High stiffness } \\
\text { and strength. }\end{array}$ & $\begin{array}{l}\text { Injection moulding. } \\
\text { Hot embossing. Laser } \\
\text { photoablation. }\end{array}$ & $\begin{array}{c}\text { (Attia, Marson and Alcock } \\
\text { 2009, Becker and Locascio } \\
\text { 2002, Fiorini and Chiu } \\
\text { 2005, Li et al. 2008) }\end{array}$ \\
\hline PTEG & $\begin{array}{l}\text { Transparent thermoplastic. Good } \\
\text { impact and chemical resistance. }\end{array}$ & $\begin{array}{l}\text { Hot embossing. Laser } \\
\text { photoablation. }\end{array}$ & $\begin{array}{l}\text { (Becker and Locascio } \\
\text { 2002, Fiorini and Chiu } \\
\text { 2005) }\end{array}$ \\
\hline PDMS & $\begin{array}{l}\text { Transparent elastomeric polymer. } \\
\text { Biocompatibility. High flexibility. } \\
\text { High gas permeability. UV } \\
\text { resistance. Chemically inert. } \\
\text { Thermally stable. }\end{array}$ & $\begin{array}{l}\text { Soft-lithography. Direct } \\
\text { laser plotting. }\end{array}$ & $\begin{array}{l}\text { (Becker and Locascio } \\
\text { 2002, Fiorini and Chiu } \\
\text { 2005, Mata, Fleischman } \\
\text { and Roy 2005, McDonald } \\
\text { et al. 2000) }\end{array}$ \\
\hline
\end{tabular}

The selection of the polymer to be applied as a matrix in a microfluidic device has to be highly related with the required properties of the fabrication method (e.g., PMMA is an option for injection moulding, which requires a thermoplastic polymer), and also with properties for which it was designed (e.g., PDMS is a good choice if it requires a biocompatible polymer for culture cells applications).

Currently, there are a variety of methods regarding the fabrication of microfluidic devices, including injection moulding (Attia, Marson and Alcock 2009), hot embossing (Becker and Heim 2000), soft-lithography (Lima et al. 2008), direct laser plotting (Wang et al. 2012), laser photoablation or laser micromachining (Rossier, Reymond and Michel 2002), photolithography (Marchesan et al. 2013), X-ray lithography (Mappes, Achenbach and Mohr 2007), among other more recently and low-costly microfabrication techniques, such as the print-and-peel techniques, e.g., xurography (Pinto et al. 2014).

The following sub-chapters will focus on the most used techniques described in literature to fabricate microfluidic devices from polymer substrates, and also the recent low-cost techniques that are gaining interest, especially at research level.

\subsection{Hot embossing}

Hot embossing was first described in the late 1990s and some of the imprinting plastic subtracts methods are still used today (Becker and Locascio 2002). The embossing is a technique involving thermoplastic materials, such as PMMA, PC COC, PS or PETG, which are patterned against a master (stamp), which is normally silicon or metal sheets, using pressure and heat (Fiorini and Chiu 2005, Sollier et al. 2011, Becker and Locascio 2002). Although embossing is a fast and inexpensive technique, it requires dedicated press equipment and a 
robust mould, which can be time-consuming, and thus not ideal for routine microfluidic designs, such as for laboratory testing (Fiorini and Chiu 2005).

\subsection{Injection moulding}

Injection moulding is a process commonly used in the plastics industry to produce a large variety of everyday objects.

This technique was first described in 1997 by researchers at Soane Bioscience for the fabrication of microchannel devices regarding the electrophoretic separation of DNA (McCormick et al. 1997).

The injection moulding process starts with thermoplastic pellets, such as PMMA or PC, being melted and injected under high pressure into the heated mould cavity. The injected pieces are then cooled and released from the mould (Fiorini and Chiu 2005, Sollier et al. 2011). Similar to hot embossing, injection moulding is mostly applied in industry, due to the complexity of moulding equipment and fabrication of the masters, as well as initial cost of the moulding equipment and masters. However, when compared with embossing, this process offers a highthroughput fabrication option, with large-volume production (Fiorini and Chiu 2005).

\subsection{Laser photoablation}

Photoablation was first reported in the literature as a method to fabricate microfluidic channels, by Roberts and co-workers in 1997 (Roberts et al. 1997). This technique involves the use of a high-powered pulsed laser to remove material from a sheet of thermoplastic material (Fiorini and Chiu 2005). In this process, a shock wave is produced while particles are injected from the substrate, creating the microchannels geometries (Becker and Locascio 2002).

Micromachining, using a laser ablation, can be achieved either by exposing the polymer substrate with a mask that defines the area to be ablated, or using a direct-write by a maskless process (Becker and Locascio 2002). The depth of the ablated channels is dependent on the pulse rate, as well as on the substrate characteristics. ArF excimer lasers (193 nm) have been used to ablate PS, PC and polyethyleneterephthalate (PET), while KrF excimer lasers ( $248 \mathrm{~nm}$ ) have been used to PMMA, PETG, PS, PC, among others (Becker and Locascio 2002, Fiorini and Chiu 2005). $\mathrm{CO}_{2}$ lasers with wavelengths in the infrared region $(10.6 \mu \mathrm{m})$ have also been applied to PMMA or PET (Fiorini and Chiu 2005).

The main advantage of this technique, for the fabrication of microfluidic devices, is that new microfluidic designs are easily programmed into the system using a direct-write process. However, this direct-write laser ablation approach has several limitations concerning massproduction (Fiorini and Chiu 2005).

\subsection{Soft-lithography}

The term "soft-lithography" was given by Xia and Whitesides in 1998 (Xia and Whitesides 1998), as a set of techniques that includes replica moulding using elastomeric materials for the fabrication of microfluidic devices, specially PDMS, as well as for the patterning of surfaces using PDMS stamps (Fiorini and Chiu 2005).

Similar to all the other methods described so far, a master template/mask is necessary to replicate the moulds. To date, the most current mask fabrication technique used for softlithography is photolithography, due to the high resolution of the photolithographic masks, enabling the fabrication of microchannels with a few nanometers, as well as complex geometries. Thereby, after the silicon master has been made, an elastomeric polymer is casted onto silicon stamp and cured. The curing process may be performed at room temperature or at slightly elevated temperature to speed the curing process (PDMS is normally cured in the 
range of temperature between 40 and $80 \stackrel{\circ}{ }$, during 20 min to $2 \mathrm{~h}$ ). Finally, after the PDMS is cured the moulds are peeled off from the mask. The same process may be repeated to obtain hundreds of replicas.

The main advantage of this technique is the ease bonding of the PDMS moulds to plastic, elastomeric polymers or glass substrates regarding the sealing process, which can be reversible or irreversible depending on the applied sealing process. Moreover, the simplicity associated with this easy sealing procedure, has made this fabrication technology one of the most widely used in the prototyping of microfluidic systems (Becker and Locascio 2002). Indeed, soft-lithography technique using PDMS, brings several advantages including low cost, fast processing, reusability of the masters, design of complex 3-dimensional systems by multilayer fabrication, excellent optical transparency, easy installation of fluidic interconnects, as well as the applicability to a variety of biological and cellular processes due to the biocompatibility property of the polymer (Fiorini and Chiu 2005).

Regarding the elastomeric polymers used in this technique for fabrication of microfluidic devices, the majority of reports in the literature used PDMS (Becker and Locascio 2002). Nevertheless, other elastomeric polymers can also be suitable for moulding by the softlithography technique.

\subsection{X-ray lithography}

More recently, X-ray lithography is being applied for the fabrication of microfluidic channels with complex 3D structures (Romanato et al. 2004), normally obtained by layer-to-layer with lithography techniques, as well as for submicron feature size and high aspect ratios (thickness/minimum feature size, AR) (Mappes, Achenbach and Mohr 2007).

The fabrication process starts with a quartz-chrome mask generated to define the pattern. Then, a reusable gold/Kapton ${ }^{T M}$ mask for the LIGA process (German acronym for "LIthographie Galvanoformung Adformung", which means, lithography, electroplating and moulding) is also generated by coating a Kapton film with a very thin film of gold placed in contact with PMMA substrate. The Kapton layer (transparent to X-rays) is then coated with photoresist and the image from the quartz-chrome mask transferred photolithographically to the photoresist over the Kapton layer. After this process, a thick layer of gold is deposited onto the Kapton surface in the open areas of the photoresist. The X-rays are absorbed by the gold layer while the section of the Kapton without the thick gold layer is transparent to the X-rays. After that, the photoresist is removed and the polymer substrate irradiated through the gold/Kapton mask in order to degrade the exposed polymer. Finally, the degraded polymer is dissolved in a solvent that solubilizes the reaction products forming the microstructures to yield high aspect ratio structures with straight and smooth walls (Becker and Locascio 2002, Mappes, Achenbach and Mohr 2007, Romanato et al. 2004).

\subsection{Xurography}

The fabrication of microfluidic devices generally requires a mask, which will serve as mould for manufacturing the microchips.

The most popular and traditional technique for the development of this mask, is photolithography due to its main advantages, such as high-resolution capabilities, low material costs, gas permeability and optical transparency (Pinto et al. 2014, Duffy et al. 1998). However, this technique requires cleanroom environment and specialized equipment and operators, making the process expensive and also time-consuming. These drawbacks, are creating a need for the alternative low-cost techniques, especially to be applied in research institutions without dedicated facilities (Pinto et al. 2014). Furthermore, these low-cost 
Polymer microfluidic devices: An overview of fabrication methods Raquel O. Rodrigues, Rui Lima, Helder T. Gomes, Adrián M. T. Silva

techniques can reduce the time required for the design and testing of new microchip designs brought from research ideas.

Concerning this need, new nonlithographic techniques, such as xurography are gaining interest in the scientific field.

The first report using a xerographic process to produce microfluidic devices was published in 2001, by Tan and co-workers (Tan et al. 2001). This novel print-and-peel (PAP) technique, has shown to be effective and most important of all, a rapid and low-cost technique to fabricate microfluidic channels.

Xurography, uses a cutting plotter machine and adhesive foils (normally, vinyl films, but can be also performed with PET, nitrocellulose and aluminium) to generate the master moulds or mask (Focke et al. 2010, Pinto et al. 2014). The mask is moulded with elastomeric polymers, such as PDMS used in the soft-lithography technique. Moreover, this technique can be directly applied to fabricate microchannels. In both ways, this technique does not involve photolithography or cleanroom facilities, which is a great advantage.

The main disadvantage of this technique is the relatively poor resolution capabilities and micron-sizes precision. The thickness of the microchannel is also dependent on the type of foil material that is used.

\subsection{Other low-cost fabrication techniques}

Other low-cost technologies, including laser direct machining (Wang et al. 2012) or 3D printing (Erkal et al. 2014, McDonald et al. 2002), are also being used to the fabrication of microfluidic devices.

Laser direct machining, also known as direct laser plotting consists in a laser technique that was adapted to generate microchannels directly into the substrate of the microfluidic devices, normally cured PDMS or PMMA, without the need of a mask.

To create the patterns, four parameters can be adjusted, namely, laser power, pulse density (pulses per inch-PPI), focus and laser moving speed (Wang et al. 2012). This fabrication technique offers advantages such as time and cost saving over the conventional softlithography technique, eliminating the need of a cleanroom facility, as well as complex fabrication steps. Due to these advantages, laser direct machining appears to be suitable to be applied in research laboratories. However, it needs improvement in order to be useful in mass-production.

$3 D$ printing, is an adapted technique that has been recently applied to produce microfluidic devices. This techniques operates printers either by printing a thermoplastic polymer that solidifies after extrusion or by printing a binding material that joins regions of a predeposited layer (McDonald et al. 2002). Generally, the extruded thermoplastic material serves as the 3Dmask, which is further used to prototype the microfluidic devices.

Although this fabrication technique has some limitations concerning the size of the microchannels and some laborious fabrication steps for the final microfluidic devices, the further development of this 3D-printing technique will certainly become a major theme in the fabrication of microfluidic devices, due to their several advantages, such the design of complex 3D structures and rapid prototyping.

\section{Discussion and future outlook}

In the 1980s, microfluidic devices were first developed using the available and highly established fabrication techniques brought from the MEMS technology that used glass and silicon as substracts (Terry, Jerman and Angell 1979). Since then, microfabrication techniques 
have greatly evolved alongside with science materials, especially with the development of polymers. Actually, due to their many advantages, such as being a low-cost material, easier microfabrication and having a wide range of mechanical and chemical properties, polymers are perfect materials to be used in the fabrication of microfluidic devices and became, as envisioned by Whitesides (Whitesides 2006), the major theme in analysis.

Furthermore, the characteristics of polymers overcome the major issue of biomedical and clinical applications, which is the creation of a low-cost and disposable microfluidic chip, to be used as point-of-care testing.

Indeed, there are several methods reported in the literature for the fabrication of microfluidic devices using polymers as a substrate. Usually, the selection of the fabrication method will highly depend on the final goal, which for the industrial perspective, is the mass production while for a research laboratory level, is the rapid prototyping, which includes short fabrication time and low-cost for a complete cycle from design to testing (Sollier et al. 2011).

Due to these two different perspectives, industrial and laboratory level, the main advantages and disadvantages of each fabrication technique described are compiled in Table 3 for summary purposes.

\begin{tabular}{|c|c|c|}
\hline Methods & Advantages & Disadvantages \\
\hline Hot embossing & $\begin{array}{l}\text { Precise and rapid in the replication of } \\
\text { microstructures. Mass production. }\end{array}$ & $\begin{array}{l}\text { Restricted to thermoplastics. Time- } \\
\text { consuming. Complex 3D structures are } \\
\text { difficult to be fabricated. }\end{array}$ \\
\hline Injection moulding & $\begin{array}{l}\text { Mass production. Fine features. Low } \\
\text { cycle time. Highly automated. }\end{array}$ & $\begin{array}{l}\text { Restricted to thermoplastics. High cost } \\
\text { mould. Micro size precision is limited. }\end{array}$ \\
\hline Laser photoablation & Rapid. Large format production. & $\begin{array}{l}\text { Limited materials. Multiple treatment } \\
\text { session. Difficulties for mass production. } \\
\text { Micro size precision is limited. }\end{array}$ \\
\hline Soft-lithography & $\begin{array}{l}\text { High-resolution and 3D geometries. } \\
\text { Cost-effective. Excellent micro size } \\
\text { precision. }\end{array}$ & $\begin{array}{l}\text { Pattern deformation and vulnerability to } \\
\text { defects. Difficult to fabricate circular } \\
\text { geometries. }\end{array}$ \\
\hline X-ray lithography & $\begin{array}{l}\text { High-resolution. Straight and smooth } \\
\text { walls. }\end{array}$ & $\begin{array}{l}\text { Complex and difficult master fabrication. } \\
\text { Time consuming and high cost process. }\end{array}$ \\
\hline Xurography & Low-cost and rapid technique. & $\begin{array}{l}\text { Complex 3D structures are difficult to be } \\
\text { fabricated. Micro size precision is limited. }\end{array}$ \\
\hline Direct laser plotting & $\begin{array}{l}\text { Low-cost and rapid technique. Free- } \\
\text { mask technique. }\end{array}$ & $\begin{array}{l}\text { Complex 3D structures are difficult to be } \\
\text { fabricated. Micro size precision is limited. } \\
\text { Reproducibility of the microdevices. }\end{array}$ \\
\hline 3D-printing & Low-cost and rapid technique. & $\begin{array}{l}\text { Multiple treatment session. Difficulties for } \\
\text { mass production. Micro size precision is } \\
\text { limited. }\end{array}$ \\
\hline
\end{tabular}

Table 3. Main advantages and disadvantages for the fabrication techniques of the microfluidic devices based in polymer substrates. Adapted from (Wu and Gu 2011)

In a mass-production industrial perspective, hot embossing and injection moulding are in generally the most used microdevice fabrication methods. Beyond the initial cost of equipments and mask moulds, the mass-production with high precision, short replication time and the very long lifetime of the stamps, makes these techniques very attractive for industrial purposes. Therefore, these techniques are more commonly found in industrial applications than in research laboratories. 
Polymer microfluidic devices: An overview of fabrication methods Raquel O. Rodrigues, Rui Lima, Helder T. Gomes, Adrián M. T. Silva

On the other hand, research laboratories, focused their needs in rapid prototyping of microfluidic devices (as new ideas have to be quickly tested and improved), as well as in a low cost fabrication perspective.

Figure 2 shows an overview of the fabrication methods previously presented in Table 3 , in a time and cost perspective.

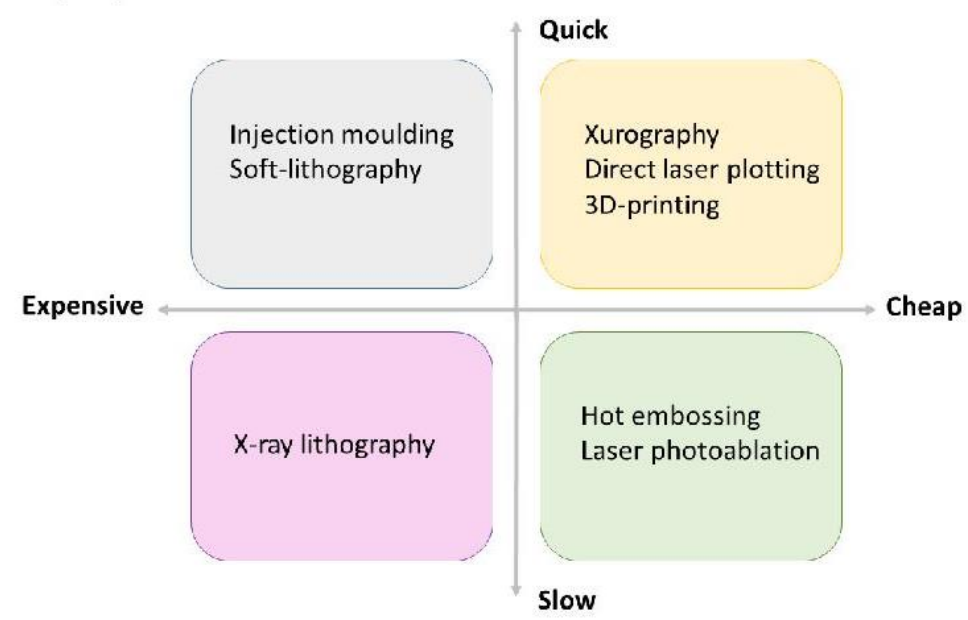

Figure 2. Overview of microfluidic fabrication techniques regarding the costs and time-consumption point-of-view

Under a research laboratory point-of-view, where the production cost and time-consumption are of utmost importance, Figure 2 clearly reveals that the recent low-cost techniques are filling a gap in the microdevice fabrication methods, concerning the rapid and inexpensive microchips for testing new ideas.

Nevertheless, it is important to bear in mind that for the research laboratory the available technologies, equipments and preferred material substrates, are also crucial issues for the selection of the microfabrication method.

Indeed, these last concerns are the main cause for the recent development of low-cost microfabrication techniques, such as xurography, direct laser plotting or even 3D-printing (there are many others).

Actually, low-cost techniques generally avoid the use of cleanroom facilities or lithographic techniques to produce a mask, or even the complete microdevice. Due to these specifications, low-cost fabrication techniques are becoming an important theme in the field, allowing a quick rise of studies and achievements in many scientific domains by spreading the fabrication of microfluidic devices to almost any research laboratory, as it can be observed in Figure 3, where it is shown a metadata analysis made in Scopus database with the search sentence, "low-cost fabrication for microfluidic devices", between 1996 (year of the first work reported in literature) and 2014. 


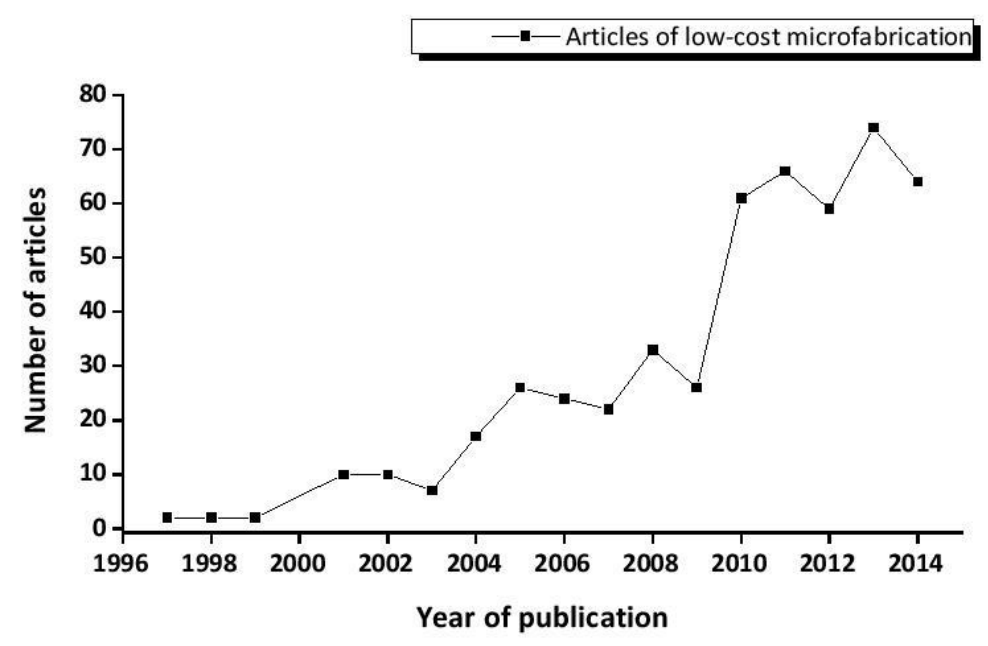

Figure 3. Number of publications concerning the low-cost microfabrication techniques since 1996 to 2014

The exponential growth of published documents concerning low-cost fabrication techniques, mainly articles, conference papers and reviews, is shown in Figure 3. Mainly after 2009 where the number of published documents have triplicated, proving the growing interest from the scientific community for these alternative and low-cost microfabrication techniques.

However, these recent low-cost techniques still need improvements, especially concerning the micro size precision, complex 3-D microchannels designs and reproducibility of the microdevices. Nevertheless, and despite these limitations, low-cost techniques are still representing for research laboratories an important tool to be used as screening experiments, in order to test, discard and improve microchannels designs that can be further developed with other more traditional and improved microfabrication techniques, such as photolithography or even X-ray lithography.

Considering the three past decades, it seems to be obvious that with the growing interest for low-cost microfabrication techniques by the scientific community, the limitations will be overcome in a near future. Therefore, these techniques will probably became a major theme concerning the fabrication techniques for microfluidic devices having a massive impact in the worldwide utilization of microdevices in many scientific fields.

\section{References}

Attia, Usama M, Silvia Marson and Jeffrey R Alcock. 2009. "Micro-injection moulding of polymer microfluidic devices." Microfluidics and Nanofluidics no. 7 (1):1-28. DOI: 10.1007/s10404-009-0421-x.

Becker, Holger and Ulf Heim. 2000. "Hot embossing as a method for the fabrication of polymer high aspect ratio structures." Sensors and Actuators A: Physical no. 83 (1-3):130-135. DOI: 10.1016/S0924-4247(00)00296-X.

Becker, Holger and Laurie E. Locascio. 2002. "Polymer microfluidic devices." Talanta no. 56 (2):267287. DOI: S0039-9140(01)00594-X.

Catalano, Rossella, Gerardo Perozziello, Giuseppina Simone, Patrizio Candeloro, Francesco Gentile, Maria Laura Coluccio, Francesca Pardeo, Manfred Burghammer, Giovanni Cuda, Christian Riekel and Enzo Di Fabrizio. 2014. "Optimized fabrication protocols of microfluidic devices for X-ray analysis." Microelectronic Engineering no. 124:13-16. DOI: 10.1016/j.mee.2014.04.016. 
Daikuzono, Cristiane M., Cleber A. R. Dantas, Diogo Volpati, Carlos J. L. Constantino, Maria H. O. Piazzetta, Angelo L. Gobbi, David M. Taylor, Osvaldo N. Oliveira Jr and Antonio Riul Jr. 2015. "Microfluidic electronic tongue." Sensors and Actuators B: Chemical no. 207, Part B:1129-1135. DOI: 10.1016/j.snb.2014.09.112.

Do, Jaephil, Sehwan Lee, Jungyup Han, Junhai Kai, Chien-Chong Hong, Chuan Gao, Joseph H. Nevin, Gregory Beaucage and Chong H. Ahn. 2008. "Development of functional lab-on-a-chip on polymer for point-of-care testing of metabolic parameters." Lab on a Chip no. 8 (12):2113-2120. DOI: 10.1039/B811169C.

Duffy, David C., J. Cooper McDonald, Olivier J. A. Schueller and George M. Whitesides. 1998. "Rapid Prototyping of Microfluidic Systems in Poly(dimethylsiloxane)." Analytical Chemistry no. 70 (23):4974-4984. DOI: 10.1021/ac980656z.

Erkal, Jayda L., Asmira Selimovic, Bethany C. Gross, Sarah Y. Lockwood, Eric L. Walton, Stephen McNamara, R. Scott Martin and Dana M. Spence. 2014. "3D printed microfluidic devices with integrated versatile and reusable electrodes." Lab on a Chip no. 14 (12):2023-2032. DOI: 10.1039/C4LC00171K.

Fiorini, G. S. and D. T. Chiu. 2005. "Disposable microfluidic devices: fabrication, function, and application." Biotechniques no. 38 (3):429-46. http://www.ncbi.nlm.nih.gov/pubmed/15786809.

Focke, Maximilian, Dominique Kosse, Claas Muller, Holger Reinecke, Roland Zengerle and Felix von Stetten. 2010. "Lab-on-a-Foil: microfluidics on thin and flexible films." Lab on a Chip no. 10 (11):1365-1386. DOI: 10.1039/C001195A.

Fronczek, Christopher F., David J. You and Jeong-Yeol Yoon. 2013. "Single-pipetting microfluidic assay device for rapid detection of Salmonella from poultry package." Biosensors and Bioelectronics no. 40 (1):342-349. DOI: 10.1016/j.bios.2012.07.076.

Halldorsson, Skarphedinn, Edinson Lucumi, Rafael Gómez-Sjöberg and Ronan M. T. Fleming. 2015. "Advantages and challenges of microfluidic cell culture in polydimethylsiloxane devices." Biosensors and Bioelectronics no. 63:218-231. DOI: 10.1016/j.bios.2014.07.029.

Jeong, Heon-Ho, Seong-Geun Jeong, Aeri Park, Sung-Chan Jang, Soon Gyu Hong and Chang-Soo Lee. 2014. "Effect of temperature on biofilm formation by Antarctic marine bacteria in a microfluidic device." Analytical Biochemistry no. 446:90-95. DOI: 10.1016/j.ab.2013.10.027.

Khanarian, G. and Hoechst Celanese. 2001. "Optical properties of cyclic olefin copolymers." Optical Engineering no. 40 (6):1024-1029. DOI: 10.1117/1.1369411.

$\mathrm{Li}$, Shiguang, Zhiguang Xu, Aaron Mazzeo, Daniel J. Burns, Gang Fu, Matthew Dirckx, Vijay Shilpiekandula, Xing Chen, Nimai C. Nayak, Eehern Wong, Soon Fatt Yoon, Zhong Ping Fang, Kamal Youcef-Toumi, David Hardt, Shu Beng Tor, Chee Yoon Yue and Jung-Hoon Chun. 2008. "Review of production of microfluidic devices: material, manufacturing and metrology." no. 6993:69930F69930F-12. DOI: 10.1117/12.781942.

Lima, R., S. Wada, S. Tanaka, M. Takeda, T. Ishikawa, K. Tsubota, Y. Imai and T. Yamaguchi. 2008. "In vitro blood flow in a rectangular PDMS microchannel: experimental observations using a confocal micro-PIV system." Biomed Microdevices no. 10 (2):153-67. DOI: 10.1007/s10544-007-9121-z.

Liu, Jikun, Bingchen Du, Panhe Zhang, Mohan Haleyurgirisetty, Jiangqin Zhao, Viswanath Ragupathy, Sherwin Lee, Don L. DeVoe and Indira K. Hewlett. 2014. "Development of a microchip Europium nanoparticle immunoassay for sensitive point-of-care HIV detection." Biosensors and Bioelectronics no. 61:177-183. DOI: 10.1016/j.bios.2014.04.057.

Manz, A., N. Graber and H. M. Widmer. 1990. "Miniaturized total chemical analysis systems: A novel concept for chemical sensing." Sensors and Actuators B: Chemical no. 1 (1-6):244-248. DOI: 10.1016/0925-4005(90)80209-I.

Mappes, Timo, Sven Achenbach and Juergen Mohr. 2007. "X-ray lithography for devices with high aspect ratio polymer submicron structures." Microelectronic Engineering no. 84 (5-8):1235-1239. DOI: 10.1016/j.mee.2007.01.154. 
Marchesan, Silvia, Christopher D. Easton, Katie E. Styan, Patrick Leech, Thomas R. Gengenbach, John S. Forsythe and Patrick G. Hartley. 2013. "SU-8 photolithography on reactive plasma thin-films: coated microwells for peptide display." Colloids and Surfaces B: Biointerfaces no. 108:313-321. DOI: 10.1016/j.colsurfb.2013.03.018.

Mata, Alvaro, AaronJ Fleischman and Shuvo Roy. 2005. "Characterization of Polydimethylsiloxane (PDMS) Properties for Biomedical Micro/Nanosystems." Biomedical Microdevices no. 7 (4):281-293. DOI: 10.1007/s10544-005-6070-2.

McCormick, R. M., R. J. Nelson, M. G. Alonso-Amigo, D. J. Benvegnu and H. H. Hooper. 1997. "Microchannel electrophoretic separations of DNA in injection-molded plastic substrates." Analytical Chemistry no. 69 (14):2626-30. DOI: 10.1021/ac9701997.

McDonald, J. C., D. C. Duffy, J. R. Anderson, D. T. Chiu, H. Wu, O. J. Schueller and G. M. Whitesides. 2000. "Fabrication of microfluidic systems in poly(dimethylsiloxane)." Electrophoresis no. 21 (1):2740. DOI: 10.1002/(sici)1522-2683(20000101)21:1<27::aid-elps27>3.0.co;2-c.

McDonald, J. Cooper, Michael L. Chabinyc, Steven J. Metallo, Janelle R. Anderson, Abraham D. Stroock and George M. Whitesides. 2002. "Prototyping of Microfluidic Devices in Poly(dimethylsiloxane) Using Solid-Object Printing." Analytical Chemistry no. 74 (7):1537-1545. DOI: 10.1021/ac010938q.

Mehta, A., H. Shekhar, S. H. Hyun, S. Hong and H. J. Cho. 2006. "A micromachined electrochemical sensor for free chlorine monitoring in drinking water." Water Sci Technol no. 53 (4-5):403-10. DOI: 10.2166/wst.2006.146.

Monošík, Rastislav and Lúcio Angnes. 2015. "Utilisation of micro- and nanoscaled materials in microfluidic analytical devices." Microchemical Journal no. 119:159-168. DOI: 10.1016/j.microc.2014.12.003.

Nason, F., E. Morganti, C. Collini, C. Ress, S. Bersini, G. Pennati, F. Boschetti, A. Colombini, G. Lombardi, G. Banfi, L. Lorenzelli and G. Dubini. 2011. "Design of microfluidic devices for drug screening on invitro cells for osteoporosis therapies." Microelectronic Engineering no. 88 (8):1801-1806. DOI: 10.1016/j.mee.2011.02.115.

Novo, P., V. Chu and J. P. Conde. 2014. "Integrated optical detection of autonomous capillary microfluidic immunoassays:a hand-held point-of-care prototype." Biosensors and Bioelectronics no. 57:284-291. DOI: 10.1016/j.bios.2014.02.009.

Paegel, Brian M., Robert G. Blazej and Richard A. Mathies. 2003. "Microfluidic devices for DNA sequencing: sample preparation and electrophoretic analysis." Current Opinion in Biotechnology no. 14 (1):42-50. DOI: 10.1016/S0958-1669(02)00004-6.

Pan, Xiaoyan, Lei Jiang, Kaiying Liu, Bingcheng Lin and Jianhua Qin. 2010. "A microfluidic device integrated with multichamber polymerase chain reaction and multichannel separation for genetic analysis." Analytica Chimica Acta no. 674 (1):110-115. DOI: 10.1016/j.aca.2010.06.005.

Pinto, Elmano, Vera Faustino, Raquel Rodrigues, Diana Pinho, Valdemar Garcia, João Miranda and Rui Lima. 2014. "A Rapid and Low-Cost Nonlithographic Method to Fabricate Biomedical Microdevices for Blood Flow Analysis." Micromachines no. 6 (1):121-135. DOI: 10.3390/mi6010121.

Pratt, Erica D., Chao Huang, Benjamin G. Hawkins, Jason P. Gleghorn and Brian J. Kirby. 2011. "Rare cell capture in microfluidic devices." Chemical Engineering Science no. 66 (7):1508-1522. DOI: 10.1016/j.ces.2010.09.012.

Roberts, Matthew A., Joël S. Rossier, Paul Bercier and Hubert Girault. 1997. "UV Laser Machined Polymer Substrates for the Development of Microdiagnostic Systems." Analytical Chemistry no. 69 (11):2035-2042. DOI: 10.1021/ac961038q.

Romanato, F., M. Tormen, L. Businaro, L. Vaccari, T. Stomeo, A. Passaseo and E. Di Fabrizio. 2004. "Xray lithography for 3D microfluidic applications." Microelectronic Engineering no. 73-74:870-875. DOI: 10.1016/j.mee.2004.03.067. 
Polymer microfluidic devices: An overview of fabrication methods

Raquel O. Rodrigues, Rui Lima, Helder T. Gomes, Adrián M. T. Silva

Rossier, J., F. Reymond and P. E. Michel. 2002. "Polymer microfluidic chips for electrochemical and biochemical analyses." Electrophoresis no. 23 (6):858-67. DOI: 10.1002/15222683(200203)23:6<858::aid-elps858>3.0.c0;2-3.

Shi, Jian, Li Liu and Yong Chen. 2011. "Investigation of cell culture in microfluidic devices with different bi-layer substrates." Microelectronic Engineering no. 88 (8):1693-1697. DOI: 10.1016/j.mee.2011.01.047.

Sia, S. K. and G. M. Whitesides. 2003. "Microfluidic devices fabricated in poly(dimethylsiloxane) for biological studies." Electrophoresis no. 24 (21):3563-76. DOI: 10.1002/elps.200305584.

Sollier, E., C. Murray, P. Maoddi and D. Di Carlo. 2011. "Rapid prototyping polymers for microfluidic devices and high pressure injections." Lab on a Chip no. 11 (22):3752-65. DOI: 10.1039/c1lc20514e.

Sun, Guoqiang, Panpan Wang, Shenguang Ge, Lei Ge, Jinghua Yu and Mei Yan. 2014. "Photoelectrochemical sensor for pentachlorophenol on microfluidic paper-based analytical device based on the molecular imprinting technique." Biosensors and Bioelectronics no. 56:97-103. DOI: 10.1016/j.bios.2014.01.001.

Tan, Aimin, Kenneth Rodgers, John P. Murrihy, Cian O'Mathuna and Jeremy D. Glennon. 2001. "Rapid fabrication of microfluidic devices in poly(dimethylsiloxane) by photocopying." Lab on a Chip no. 1 (1):7-9. DOI: 10.1039/B102905N.

Terry, S. C., J. H. Jerman and J. B. Angell. 1979. "A gas chromatographic air analyzer fabricated on a silicon wafer." Electron Devices, IEEE Transactions on no. 26 (12):1880-1886. DOI: 10.1109/TED.1979.19791.

van Reenen, Alexander, Arthur M. de Jong, Jaap M. J. den Toonder and Menno W. J. Prins. 2014. "Integrated lab-on-chip biosensing systems based on magnetic particle actuation - a comprehensive review." Lab on a Chip no. 14 (12):1966-1986. DOI: 10.1039/C3LC51454D.

Wang, Limu, Rimantas Kodzius, Xin Yi, Shunbo Li, Yu Sanna Hui and Weijia Wen. 2012. "Prototyping chips in minutes: Direct Laser Plotting (DLP) of functional microfluidic structures." Sensors and Actuators B: Chemical no. 168:214-222. DOI: 10.1016/j.snb.2012.04.011.

Whitesides, George M. 2006. "The origins and the future of microfluidics." Nature no. 442 (7101):368373. DOI: $10.1038 /$ nature05058.

Wu, Jing and Min Gu. 2011. "Microfluidic sensing: state of the art fabrication and detection techniques." Journal of Biomedical Optics no. 16 (8):080901-080901-12. DOI: 10.1117/1.3607430.

Xia, Younan and George M. Whitesides. 1998. "Soft Lithography." Angewandte Chemie International Edition no. 37 (5):550-575. DOI: 10.1002/(SICI)1521-3773(19980316)37:5<550::AID-ANIE550>3.0.CO;2-G.

Yager, Paul, Thayne Edwards, Elain Fu, Kristen Helton, Kjell Nelson, Milton R. Tam and Bernhard H. Weigl. 2006. "Microfluidic diagnostic technologies for global public health." Nature no. 442 (7101):412-418. DOI: 10.1038/nature05064.

Zhang, Yali, Peng Zuo and Bang-Ce Ye. "A low-cost and simple paper-based microfluidic device for simultaneous multiplex determination of different Types of chemical contaminants in food." Biosensors and Bioelectronics. DOI: 10.1016/j.bios.2014.12.042.

\section{Acknowledgments}

Work supported by project PTDC/SAU-ENB/116929/2010, EXPL/EMS-SIS/2215/2013 and UID/EQU/50020/2013, co-financed by FEDER through COMPETE, QREN and ON2, and by FCT - Fundação para a Ciência e a Tecnologia. R.O.R acknowledges the PhD scholarship SFRH/BD/97658/2013 granted by FCT. A.M.T.S acknowledges the FCT Investigator 2013 Programme (IF/01501/2013), with financing from the European Social Fund and the Human Potential Operational Programme. 


\section{Publications}

\section{D.1 International peer reviewed ISI indexed journals}

[13] Rodrigues, R.O., Baldi, G., Doumett, S., Gallo, J., Bañobre-López, M., Drazic, G., Calhelha, R., Ferreira, I.C.F.R, Lima, R., Silva, A.M.T., Gomes, H.T. (2018) "A tailor-made protocol to synthesize graphene-based yolk-shell magnetic nanoparticles for nanomedicine", C - Journal of Carbon Research, 4(4):55. DOI:10.3390/c4040055

[12] Mandla, S, Kerr-Phillips, T., Fallahi, A., Seo, J., Samanpour, R., Rodrigues, R.O., Hussain, M.A., Lee, C.K., Travas-Sejdic, J., Khademhosseinia, A., Shin, S.R. (2018) "Flexible and Stretchable PEDOT-coated Hybrid Substrates for Bioengineering Applications", Journal of Materials Chemistry $B$ (in review)

[11] Rodrigues, R.O., Baldi, G., Doumett, S., Garcia-Hervia, L., Gallo, J., Bañobre-López, M., Drazic, G., Calhelha, R., Ferreira, I.C.F.R, Lima, R., Gomes, H.T., Silva, A.M.T. (2018) "Multifunctional graphene-based magnetic nanocarriers for combined hyperthermia and dual stimuli-responsive drug delivery", Material Science Engineering C, 93:206-217. DOI: 10.1016/j.msec.2018.07.060

[10] Bento, D., Rodrigues, R.O., Faustino, V., Pinho, D., Fernandes, S.C, Pereira, A.I., Garcia, V., Miranda, J.M., Lima, R. (2018) “Deformation of Red Blood Cells, Air Bubbles, and Droplets in Microfluidic Devices: Flow Visualizations and Measurements", Micromachines, 9(4):151. DOI: 10.3390/mi9040151

[9] Ribeiro, R.S., Rodrigues, R.O., Silva, A.M.T, Tavares, P.B., Carvalho, A.M.C, Figueiredo, J.L., Faria, J.L., Gomes, H.T. (2017) "Hybrid magnetic graphitic nanocomposites towards catalytic wet peroxide oxidation of the liquid effluent from a mechanical biological treatment plant for municipal solid waste", Applied Catalysis B: Environmental, 219:645657. DOI:10.1016/j.apcatb.2017.08.013

[8] Rodrigues, R.O., Bañobre-López, M., Gallo, J., Tavares, P.B., Silva, A.M.T., Lima, R., Gomes, H.T. (2016) "Haemocompatibility of iron oxide nanoparticles synthesized for theranostic applications: a high-sensitivity microfluidic tool”, Journal of Nanoparticle Research, 18:194. DOI: 10.1007/s11051-016-3498-7

[7] Pinto, E., Faustino, V., Pinho, D., Rodrigues, R.O., Lima, R.A., Pereira, A.I. (2016) “Cellfree layer analysis in a polydimethysiloxane microchannel: a global approach", International Journal of Medical Engineering and Informatics (IJMEI), 8(3):196-209. DOI: 10.1504/ijmei.2016.077437 


\section{Publications}

[6] Rodrigues, R.O., Pinho, D., Bento, D., Lima, R., Ribeiro, J. (2016) “Wall expansion assessment of an intracranial aneurysm model by a 3D Digital Image Correlation System", Measurement, 88:262-270. DOI:10.1016/j.measurement.2016.03.045

[5] Pinho, D., Rodrigues, R.O., Pinho, D., Faustino, V., Lima, R. (2016) "Red blood cells radial dispersion in blood flowing through microchannels: The role of temperature", Journal of Biomechanics, 49(11):2293-8. DOI: 10.1016/j.jbiomech.2015.11.037

[4] Rodrigues, R.O., Lopes, R., Pinho, D., Pereira, A.I., Garcia, V., Gassmann, S., Sousa, P.C., Lima, R. (2016) “In Vitro Blood Flow and Cell-Free Layer in Hyperbolic Microchannels: Visualizations and Measurements", BioChip Journal, 10(1):9-15. DOI: 10.1007/s13206-0160102-2

[3] Rodrigues, R.O., Pinho, D., Faustino, V., Lima, R. (2015) “A simple microfluidic device for the deformability assessment of blood cells in a continuous flow", Biomedical Microdevices, 17:108. DOI: 10.1007/s10544-015-0014-2

[2] Rodrigues, R.O., Costa, H., Lima, R., Amaral, J.S. (2015) "Simple Methodology for the Quantitative Analysis of Fatty Acids in Human Red Blood Cells", Chromatographia, 78 (19):1271-1281. DOI: 10.1007/s10337-015-2947-2

[1] Pinto, E., Faustino, V., Rodrigues, R.O., Pinho, D., Garcia, V., Miranda, J.M., Lima, R. (2015) "A Rapid and Low-Cost Nonlithographic Method to Fabricate Biomedical microdevice for Blood Flow Analysis", Micromachines, 6:121-135. DOI: 10.3390/mi6010121

\section{D.2 National peer reviewed journals}

[1] Rodrigues, R.O., Lima, R., Gomes, H.T., Silva, A.M.T. (2015) “Polymer microfluidic devices: an overview of fabrication methods", U. Porto Journal of Engineering, 1(1):67-79. ISSN: 2183-6493 https://journalengineering.fe.up.pt/article/download/113/113

\section{3 Book chapter}

[1] Rodrigues, R.O., Gomes, H.T., Lima R., Silva, A.M.T., Rodrigues, P.J.S., Tavares, P.B., Tavares, J.M.R.S. (2015) “Thermal infrared image processing to assess heat generated by magnetic nanoparticles for hyperthermia applications". In: series Lecture Notes in Computer Science, Advances in Visual Computing, Bebis, G., et al. (Eds), Springer, Part 1, vol. 9474, p.25-34. Print ISBN: 978-3-319-27856-8, Online ISBN: 978-3-319-27857-5 https: / /bibliotecadigital.ipb.pt/handle/10198/13024 


\section{E. Communications in scientific meetings}

\section{E.1 Poster presentations}

[10] Rodrigues, R.O., Baldi, G., Doumett, S., Bañobre-López, M., Gallo, J., Brazic, G., Lima, R., Silva, A.M.T., Gomes, H.T. (2017),"Graphene-based magnetic nanoparticles: a dual stimuli-responsive drug release for cancer therapy". Encontro Ciência 2017, Lisboa, Portugal [9] Rodrigues, R.O., Baldi, G., Calhelha, R.C., Ferreira, I.C.F.R., Silva, A.M.T., Lima, R., Gomes, H.T. (2017), "Multifunctional graphene-based magnetic nanocarriers optimized with copolymer Pluronic F127 for biomedical applications". XXIII Encontro Galego-Portugués de Química, Ferrol, Spain

[8] Rodrigues, R.O., Baldi, G., Doumett, S., Bañobre-López, M., Gallo, J., Lima, R., Silva, A.M.T., Gomes, H.T. (2017) "Graphene-based magnetic nanoparticles as dual stimuliresponsive drug release", I Reunião do Grupo do Carbono, Porto, Portugal http://1rgc.eventos.chemistry.pt/abstract/livro.pdf

[7] Oliveira, J.R.P., Rodrigues, R.O., Marchesi, L.F., Ferreira, I.C.F.R., Gomes, H.T. (2017) "Optimization of carbon-based magnetic drug nanocarriers synthesized by green routes", I Reunião do Grupo do Carbono, Porto, Portugal http://1rgc.eventos.chemistry.pt/abstract/livro.pdf

[6] Ribeiro, R.S., Rodrigues, R.O., Silva, A.M.T., Tavares, P.B., Figueiredo, J.L., Faria, J.L., Gomes, H.T. (2017) “Core-shell magnetic carbon nanocomposites for catalytic wet peroxide oxidation", I Reunião do Grupo do Carbono, Porto, Portugal

http://1rgc.eventos.chemistry.pt/abstract/livro.pdf

[5] Rodrigues, R.O., Doumett, S., Baldi, G., Bañobre-López, M., Gallo, J., Lima, R., Silva, A.M.T., Gomes, H.T. (2016) "Development of highly hydrophilic yolk-shell Fe304@C magnetic nanoparticles for cancer theranostics", 6th EuCheMS, Seville, Spain https://bibliotecadigital.ipb.pt/handle/10198/13748

[4] Rodrigues, R.O., Gomes, H.T., Lima, R., Silva, A.M.T. (2015) “Development of the Ph.D. thesis: magnetic carbon nanostructures and study of their transport in microfluidic devices for hyperthermia", COST Action TD1402 - RADIOMAG: Medical requirements for magnetic hyperthermia application, Lisbon, Portugal

[3] Faustino, V., Rodrigues, R.O., Pinho, D., Minas, G., Lima, R. (2014) “A microfluidic device to perform blood cells separation and deformability assessment by using a high-speed video microscopy system". COST MP1205 General Meeting and Conference Advances in Optofluidics, Porto, Portugal 
[2] Costa, H., Rodrigues, R., Lima, R., Amaral, J.S. (2014) “Otimização de uma metodologia para a determinação da composição em ácidos gordos da membrana de eritrócitos por GCFID". EJI - II Encontro de Jovens Investigadores, Bragança, Portugal https: / / bibliotecadigital.ipb.pt/handle/10198/16769

[1] Costa, H., Rodrigues, R., Lima, R., Amaral, J.S. (2014) “Development of a methodology using GC-FID for the quantitative analysis of fatty acids from red blood cells". XX Encontro Luso-Galego de Química, Porto, Portugal

https: / / bibliotecadigital.ipb.pt/handle/10198/16668

\section{E.2 Oral presentations}

[6] Rodrigues, R.O., * Baldi, G., Doumett, S., Bañobre-López, M., Gallo, J., Dražić, G., Lima, R., Silva, A.M.T., Gomes, H.T. (2017) Multifunctional graphene-based yolk-shell magnetic nanoparticles assessed as $\mathrm{pH}$-dependent controlled release of anticancer drugs, In the WG1 and WG3 meeting of COST TD1402 (RADIOMAG): Ferrofluid cytotoxicity ring test with SOP for NP fabrication, Stará Lesná, Slovakia

[5] Rodrigues, R.O.*, Baldi, G., Doumett, S., Bañobre-López, M., Gallo, J., Dražić, G., Lima, R., Silva, A.M.T., Gomes, H.T. (2016) Development of stimuli-responsive graphene-based yolk-shell magnetic nanoparticles for controlled release of anticancer drugs. In the XXII Encontro Luso-Galego de Química, Bragança, Portugal https: / / bibliotecadigital.ipb.pt/handle/10198/13744

[4] Rodrigues, R.O.*, Bañobre-López, M., Gallo, J., Tavares, P.B., Silva, A.M.T., Gomes, H.T., Lima, R. (2016) A new microfluidic methodology to assess the haemocompatibility of magnetic nanoparticles designed for theranostic applications. In the annual meeting of the Cost Action MP1305: Flowing Matter, Porto, Portugal https: / /bibliotecadigital.ipb.pt/handle/10198/13019

[3] Rodrigues, R.O., Gomes, H.T., Lima, R., Silva, A.M.T., Rodrigues, P.J.S, Tavares, P.B., Tavares, J.M.R.S. * (2015) Thermal Infrared Image Processing to Assess Heat Generated by Magnetic Nanoparticles for Hyperthermia Applications. In the 11th International Symposium on Visual Computing: (1) Computer Vision (ISVC'15), Las Vegas, Nevada, USA https: / /bibliotecadigital.ipb.pt/handle/10198/13024

[2] Rodrigues, R.O.*, Bañobre-López, M., Gallo, J., Tavares, P.B., Lima, R., Silva, A.M.T., Gomes, H.T. (2015) Development and characterization of magnetic nanoparticles for theranostic applications. In the XXI Encontro Galego-Portugués de Química, Pontevedra, Spain https://bibliotecadigital.ipb.pt/handle/10198/12489 
[1] Lopes, R., Rodrigues, R.O., Pinho, D., Garcia, V., Schutte, H., Lima, R., , Gassmann, S. (2015) Low cost microfluidic device for partial cell separation: micromilling approach. In IEEE International Conference on Industrial Technology, Sevilhe, Spain https: / / bibliotecadigital.ipb.pt/handle/10198/12014

* Presenting author 


\title{
F. Grants and awards
}

\author{
Year: $\quad$ 2014-2018 \\ Grant/award: Individual Ph.D. scholarship (SFRH/BD/97658/2013) \\ Length 4 years \\ Promoting entity: $\quad$ Fundação para a Ciência e a Tecnologia (FCT)
Year: 2016
Grant/award: $\quad$ Short-Term Scientific Mission (STSM) - ECOST-STSM-TD1402-010316- 071419, CeRiCol (Vinci, Italy)
Length 45 days
Promoting entity: European Cooperation in Science and Technology, COST Action TD1402: RADIOMAG

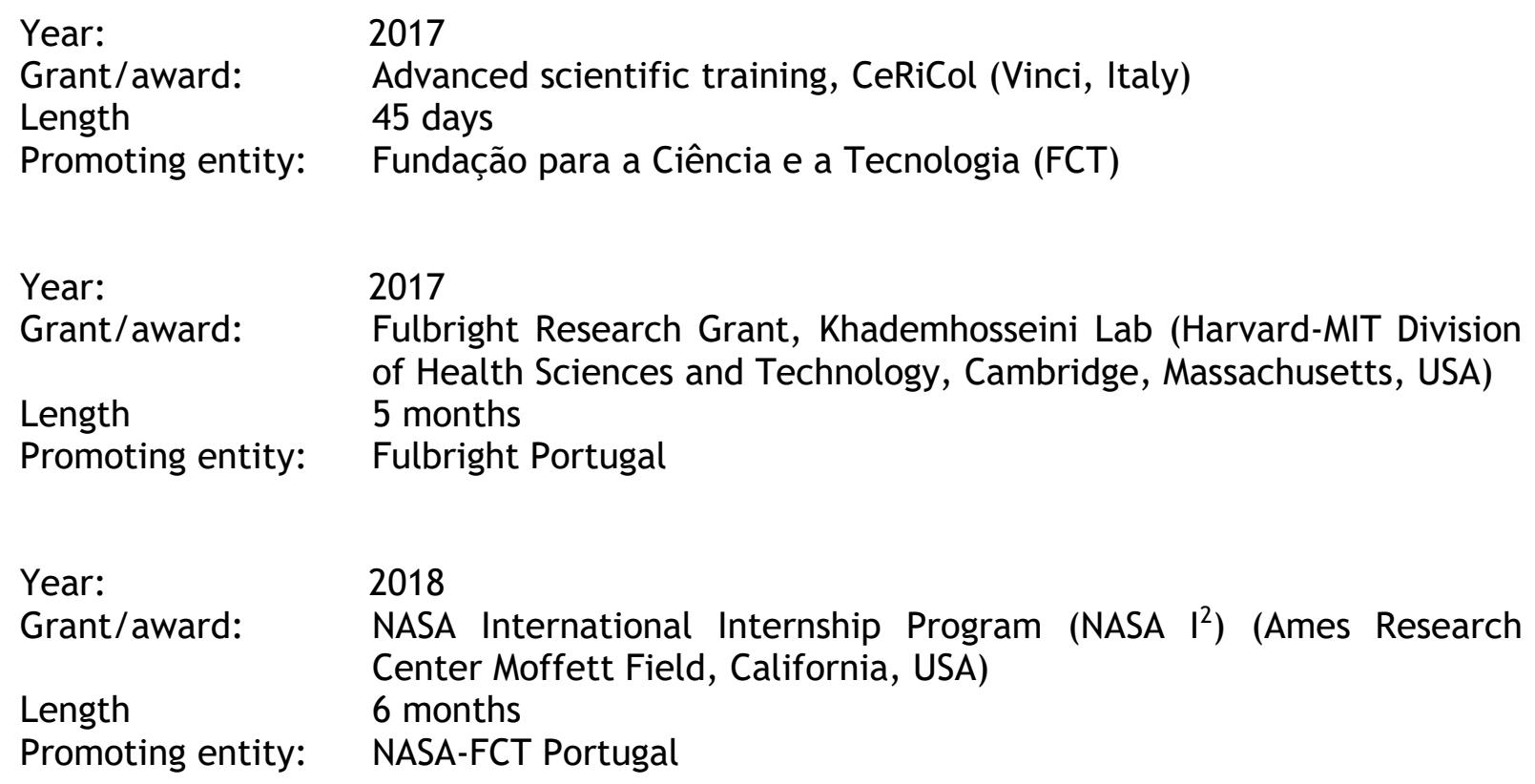

